\title{
Standards for Protection Against Radiation - 10 CFR Part 20
}

A Comparison of the Existing and Revised Rules

Manuscript Completed: June 1991

Date Published: October 1991

D. A. Cool, H. T. Peterson, Jr.

Division of Regulatory Applications

Office of Nuclear Regulatory Research

U.S. Nuclear Regulatory Commission

Washington, DC 20555
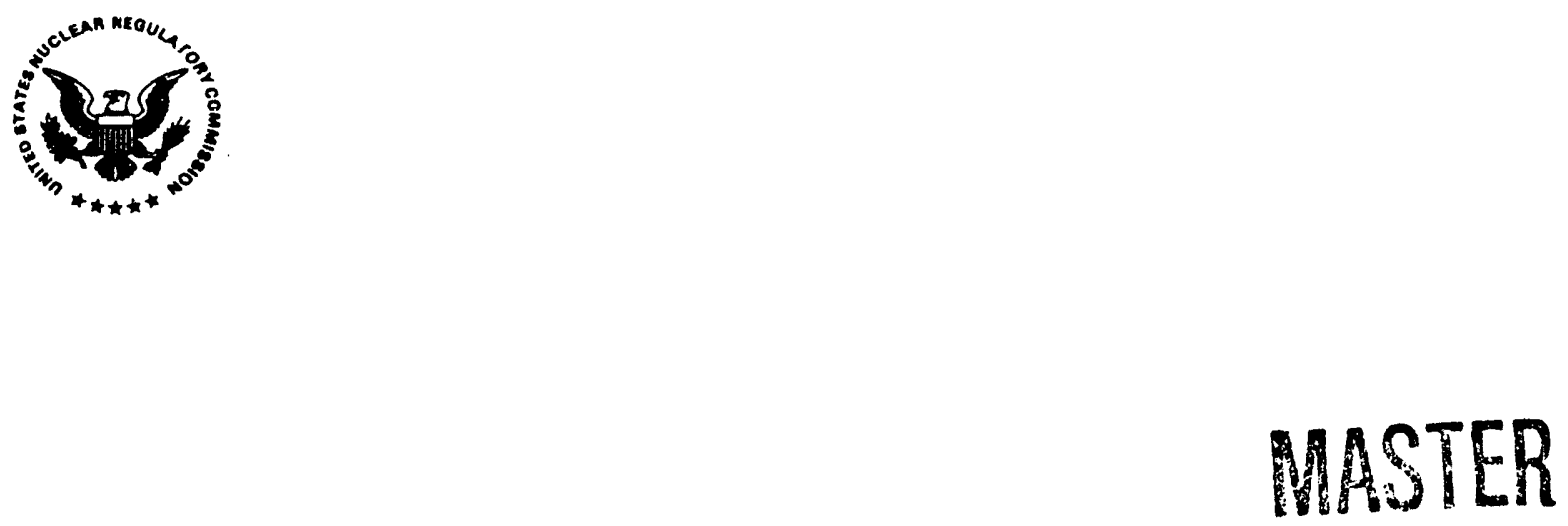


\section{ABSTRACT}

On May 21, 1991, the Nuclear Regulatory Commission (NRC) issued a revision to its standards for protection against ionizing radiation, 10 CFR Part 20. Although the revised part (\$\$ 20.1001-20.2401) became effective on June 20, 1991, I icensees may defer implementation of the revised rule until January 1, 1993. Licensees continue to be required to comply with the provisions of \$\$ 20.1-20.601 "ntil the time they adopt the provisions of \$\$20.1001-20.2401. Therefore, between June 20, 1991 and January 1, 1993 both the provisions of $\$ \$ 20.1-20.601$ and $\$ \$ 20.1001-20.2401$ are in effect. This NUREG presents a comparative text of the provisions of the revised Part 20 (\$\$ 20.1001-20.2401) to the text of $\$ \$ 20.1-20.601$ for use by the NRC staff and NRC Iicensees. 

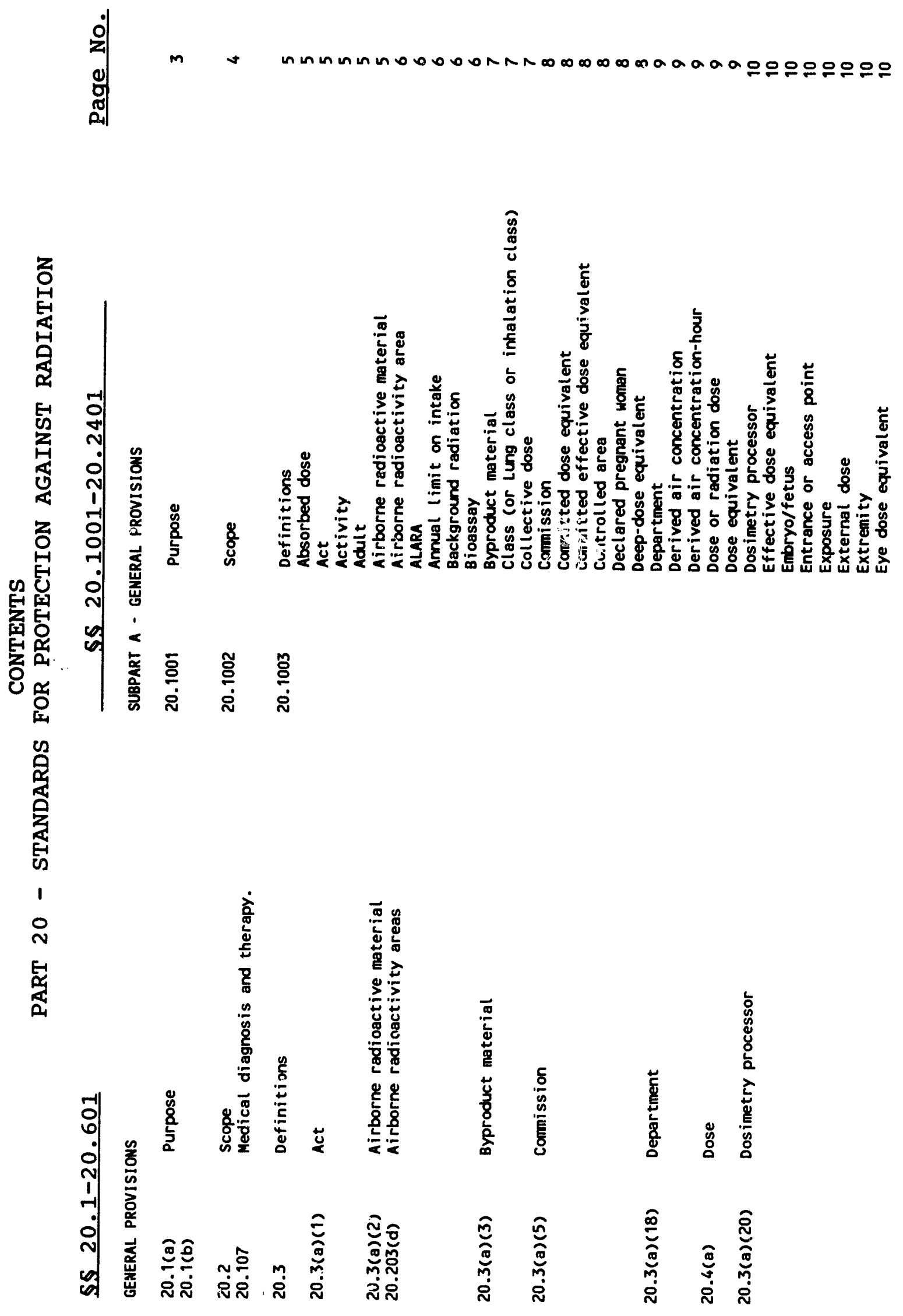


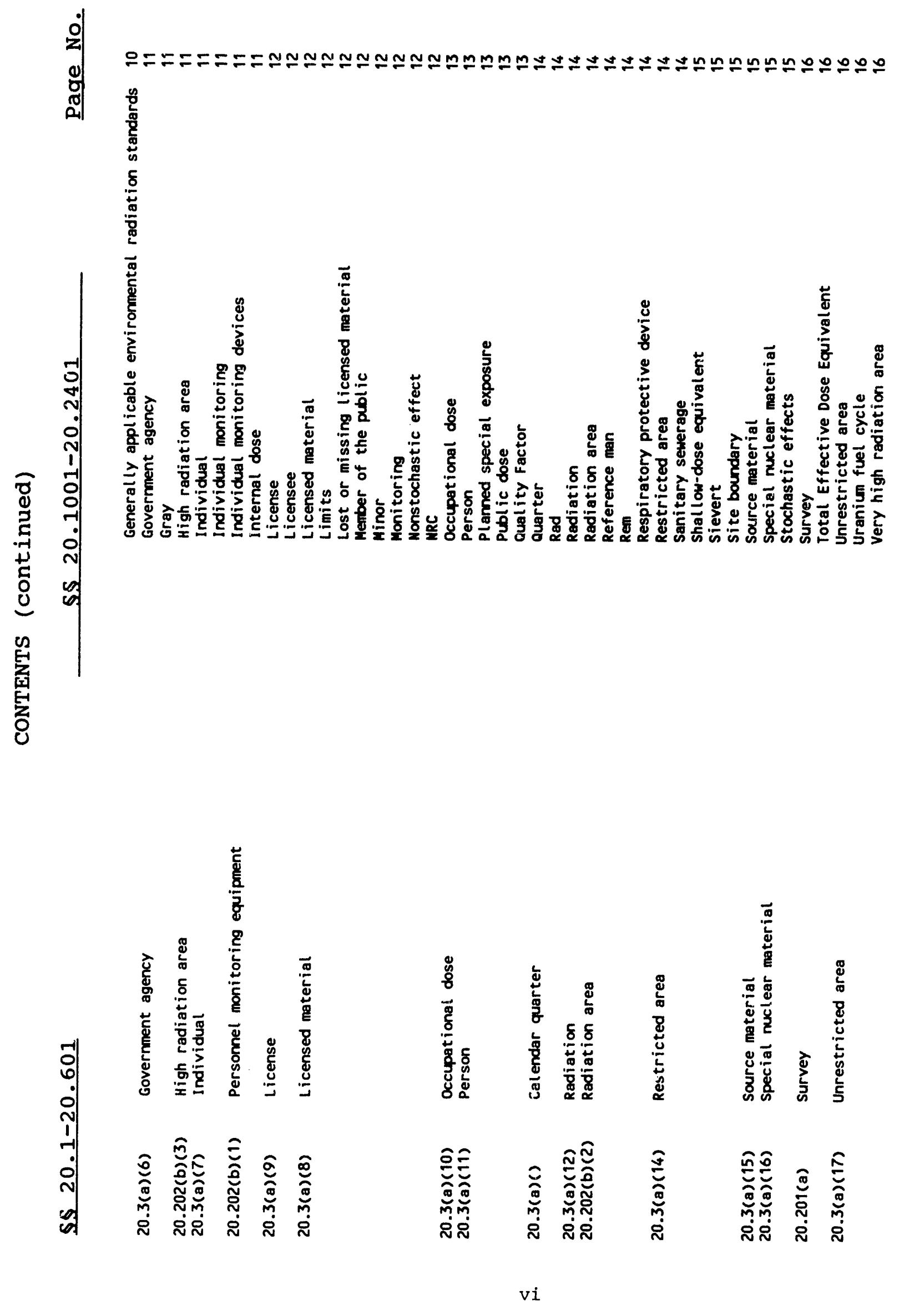




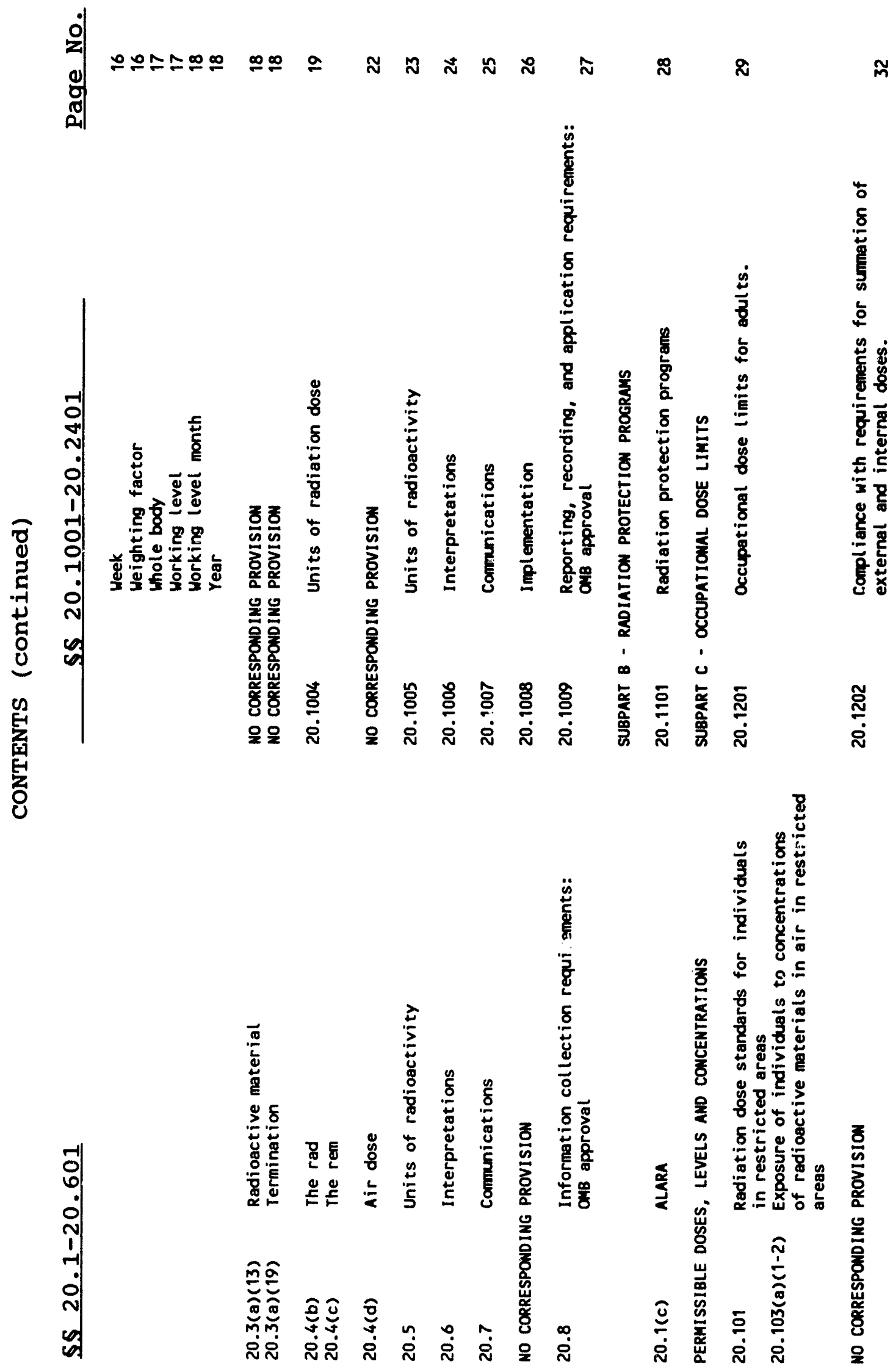




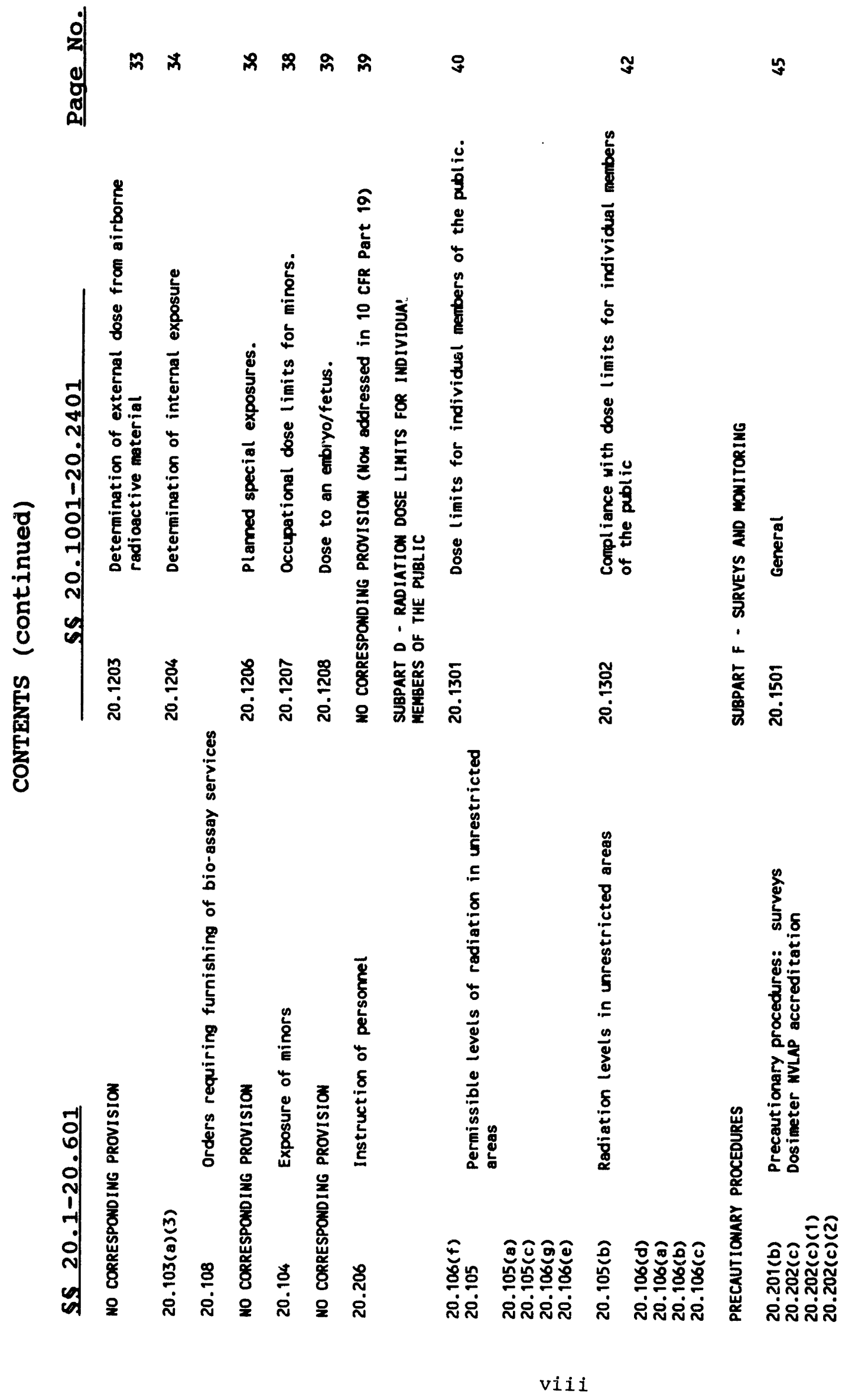




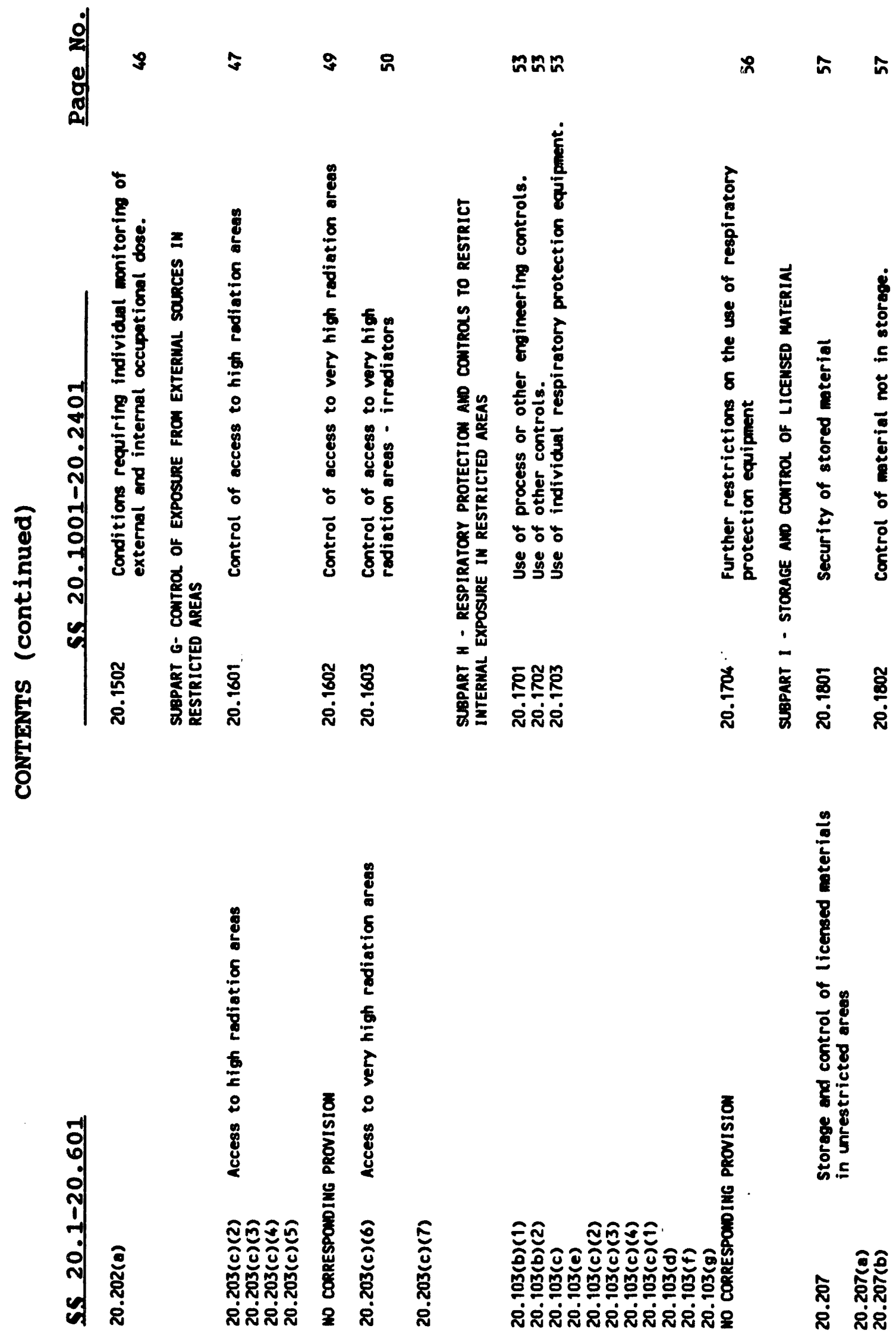




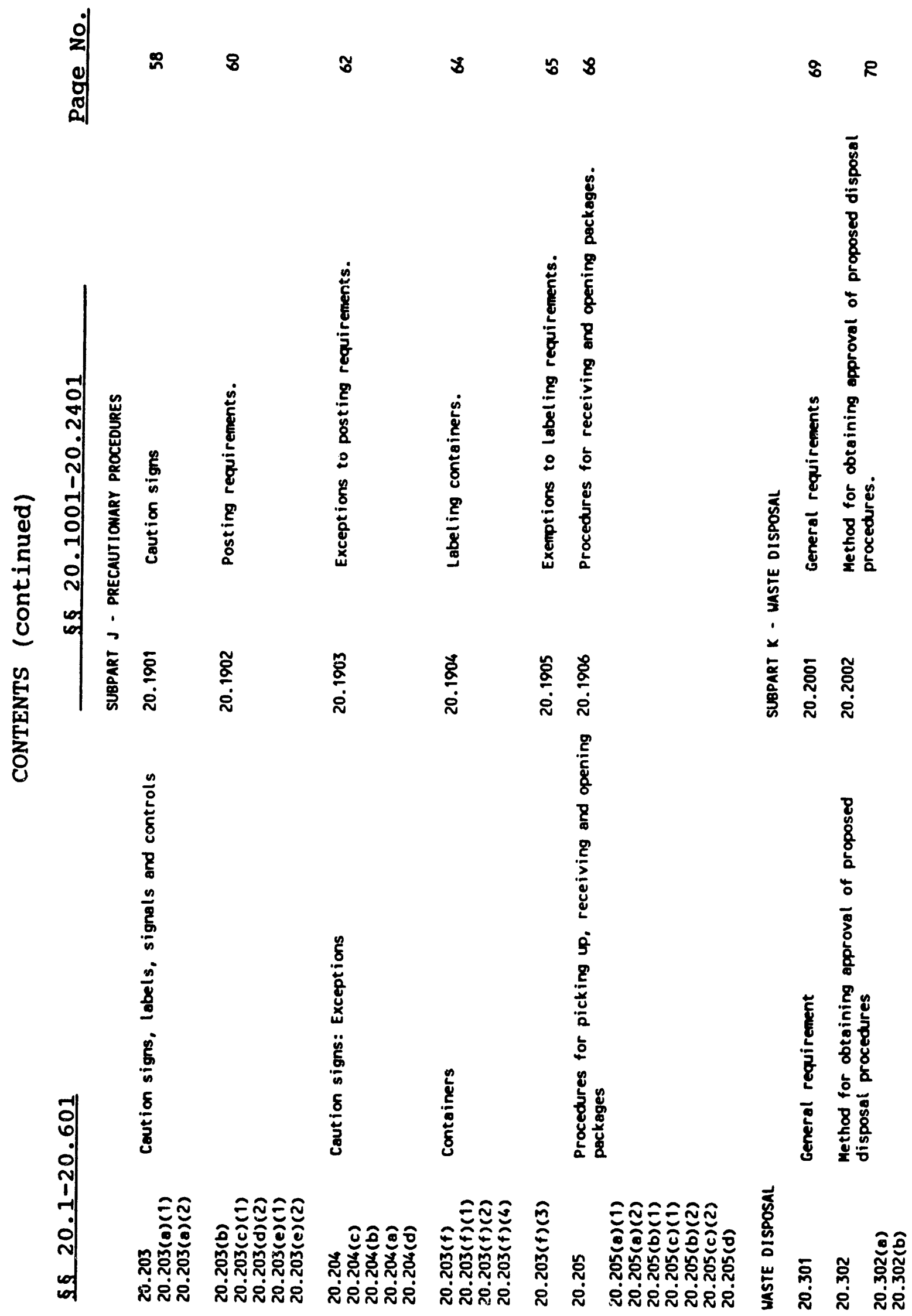




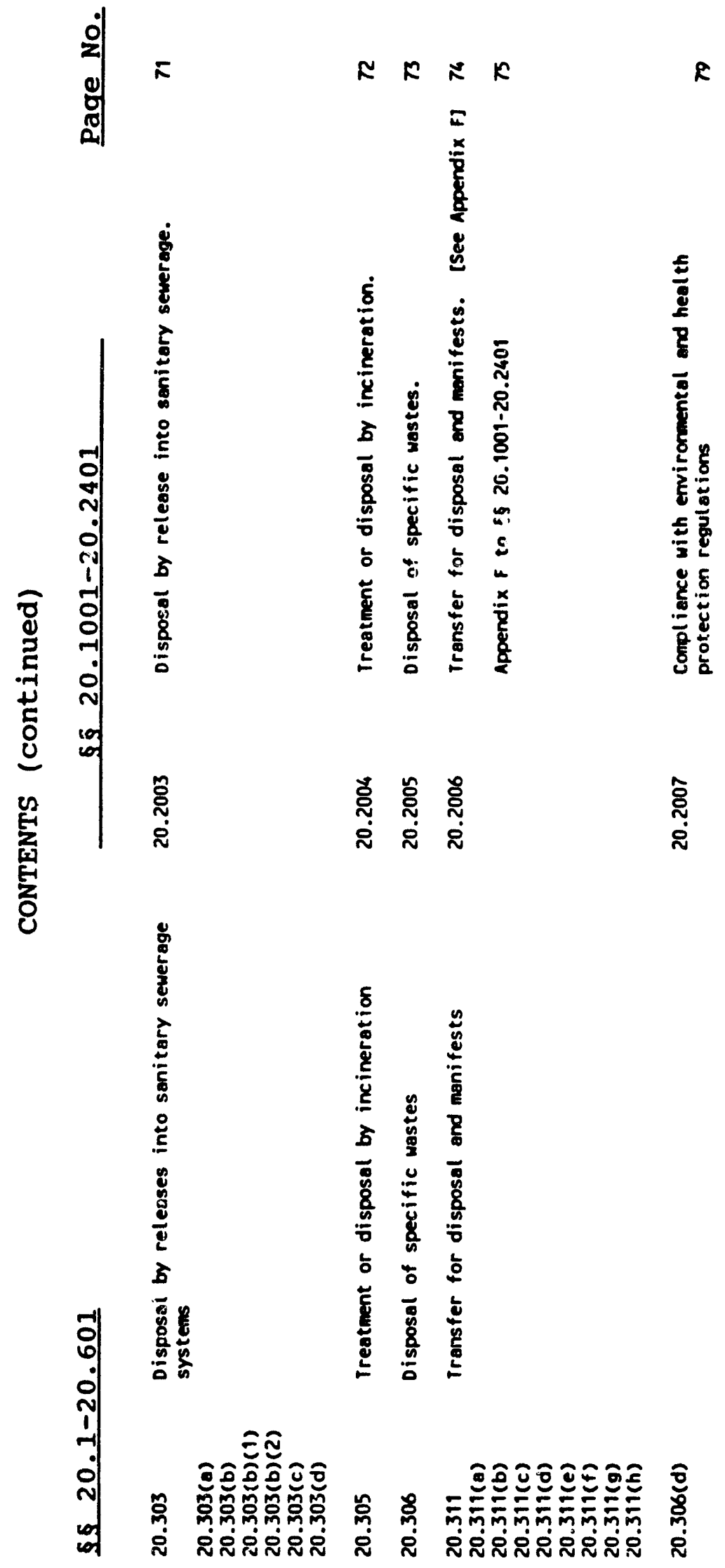




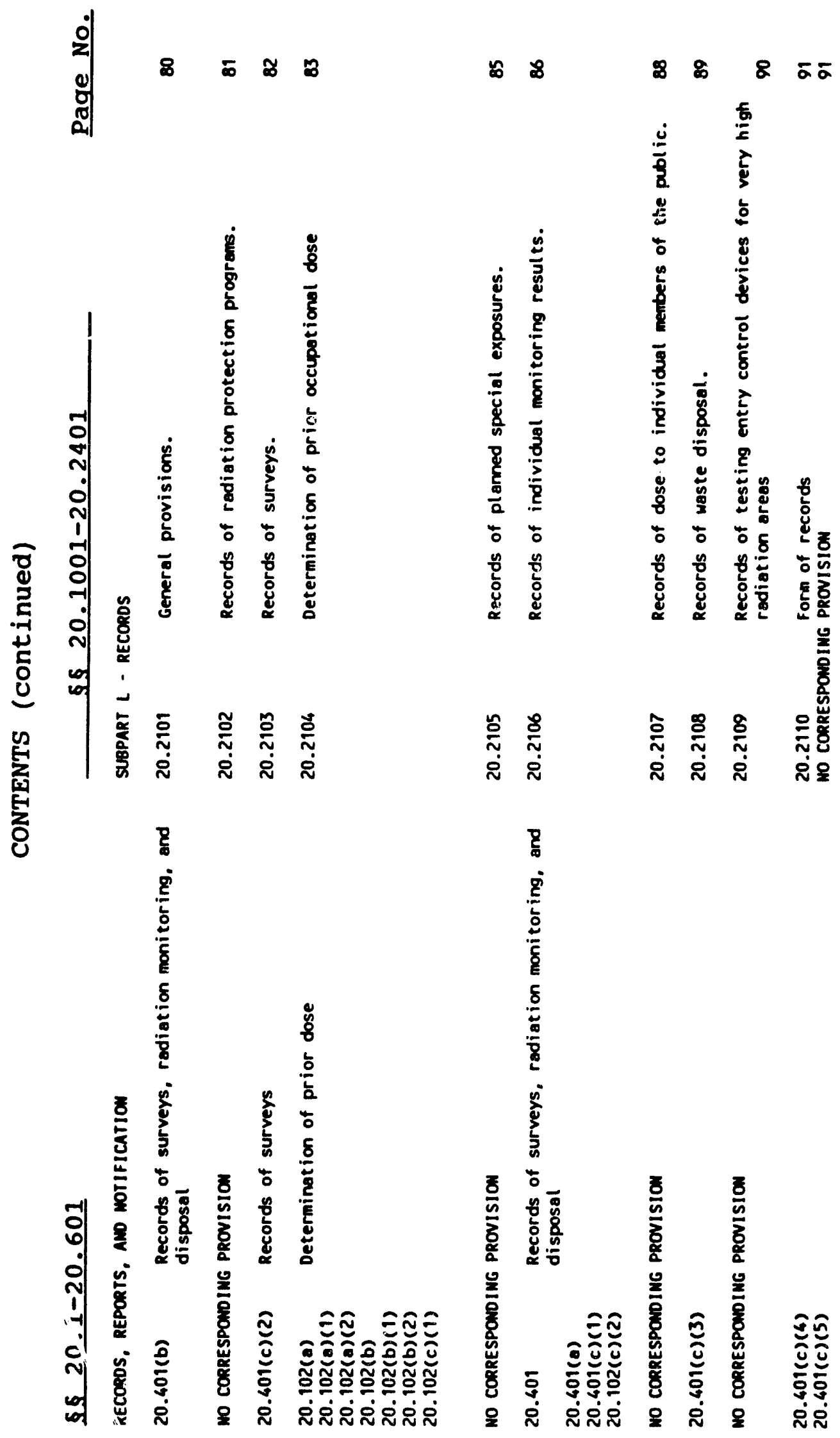




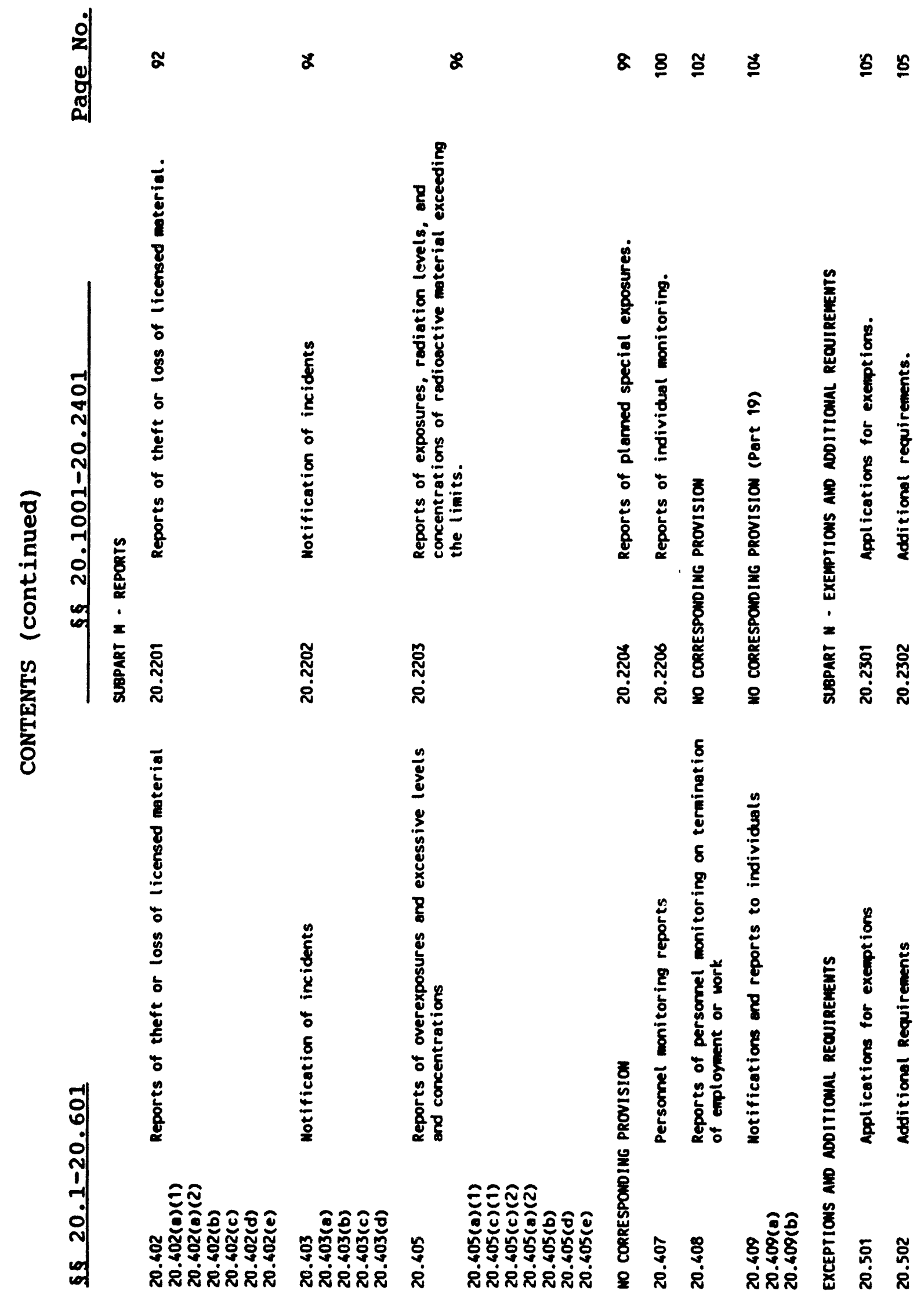



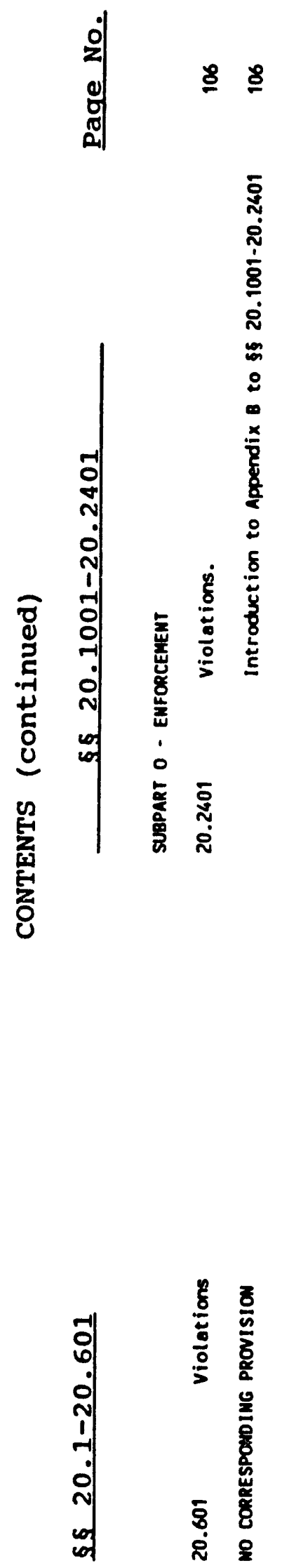

xiv 


\section{ACKNOWLEDGEMENTS}

The comparative text of the provisions of

$\$ \S 20.1-20.601$ with the provisions of

\$ 20.1001-20.2401 were prepared by

SCIENTECH, Inc. under Contract No.

NRC-04-89-067, "Technical Assistance for Part

20 Implementation." 


\section{INTRODUCTION}

The comparative text in this NUREG is organized to show the full text of each set of regulations in a side-by side format for ease of comparison. The text of $\$ \$ 20.1-20.601$ is on the left-hand side and the text of $\$ \S 20.1001-20.2401$ is on the right-hand side of each page. The sections appear in the table in numerical order according to $\$ \S 20.1001-20.2401$, with the $\$ \$ 20.1-20.601$ sections shifted and moved so that they appear opposite the corresponding sections of $\$ \S 20.1001-20.2401$. An arrow above the $\$ \$ 20.1-20.1-20.601$ provision indicates that the corresponding provision in $\$ \$ 20.1001-20.2401$ contains the same requirements as the \$\$20.1-20.601 provision. Where there is no provision in either $\$ 20.1-20.601$ or $\$ \$ 20.1001-20.2401$, this is indicated by "No Corresponding Provision" in the appropriate column. The table is arranged so that each provision appear: in its entirety in a portion of the table. Where a particular provision requires more space than that available on a single page and continues to the next, this is indicated by a note at the bottom of the page. As an additional identifying aid, the applicable section number for the text for $\$ \S 20.1001-20.2401$ is indicated at the top of each page. Footnotes appear in the table with the section or paragraph which they amplify. The presence of a footnote is indicated by "FOOTNOTE" and "END FOOTNOTE" at its beginning and end, respectively.

Since \$\$ 20.1-20.601 does not appear in numerical order in the table, an index to these sections is provided at the end of the document. This index lists these sections in numerical order and indicates the page number where each section appears in the table.

Although every effort has been made to ensure that the text of both $\S 20.1-20.601$ and $\S \S 20.1001-20.2401$ are the same as the text in the code of Federal Regulations and the Federal Register, differences may exist between the wording as it appears in the table and in the Federal Register. If there are any differences between the table in this NUREG and the actual regulation, the wording in the regulation should be used. 


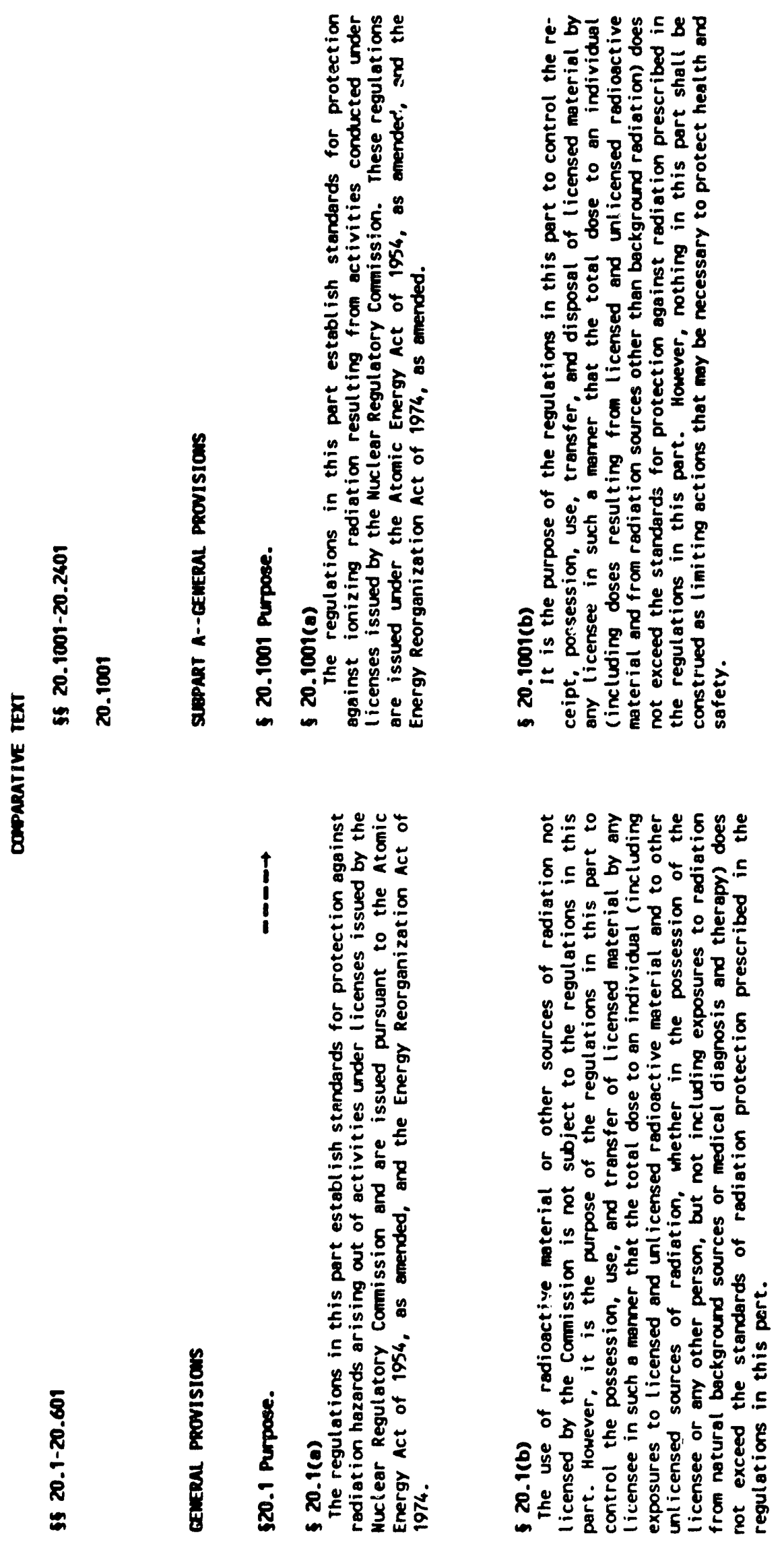



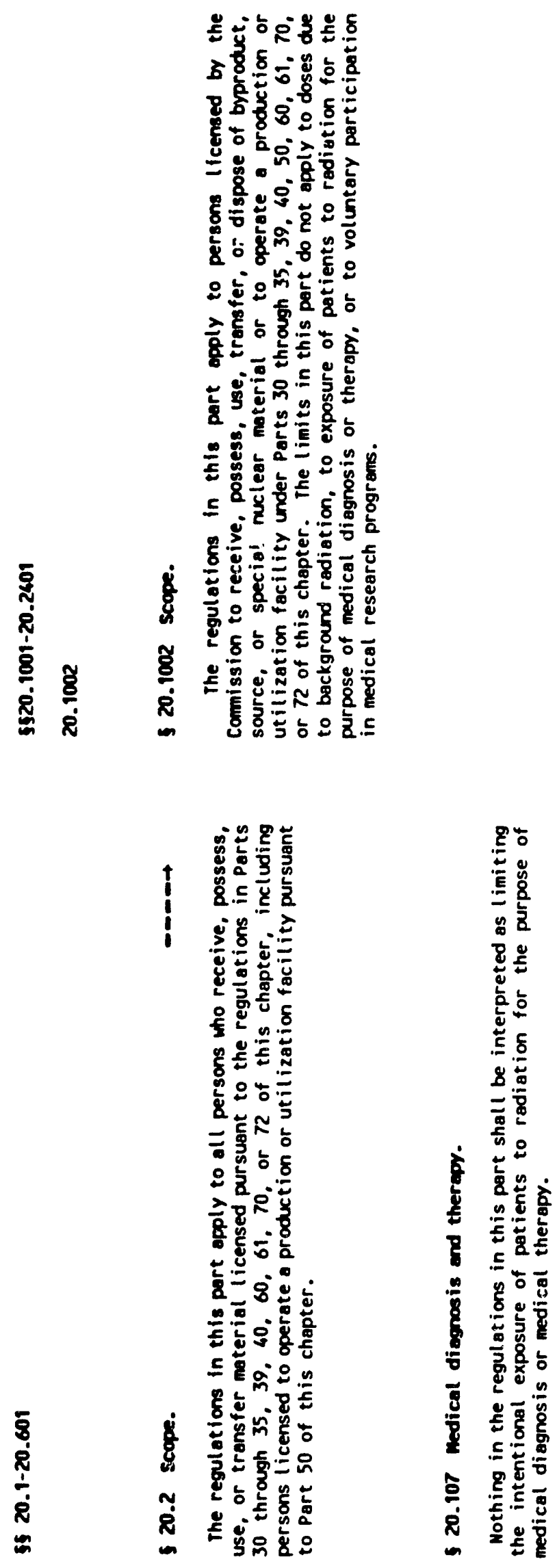


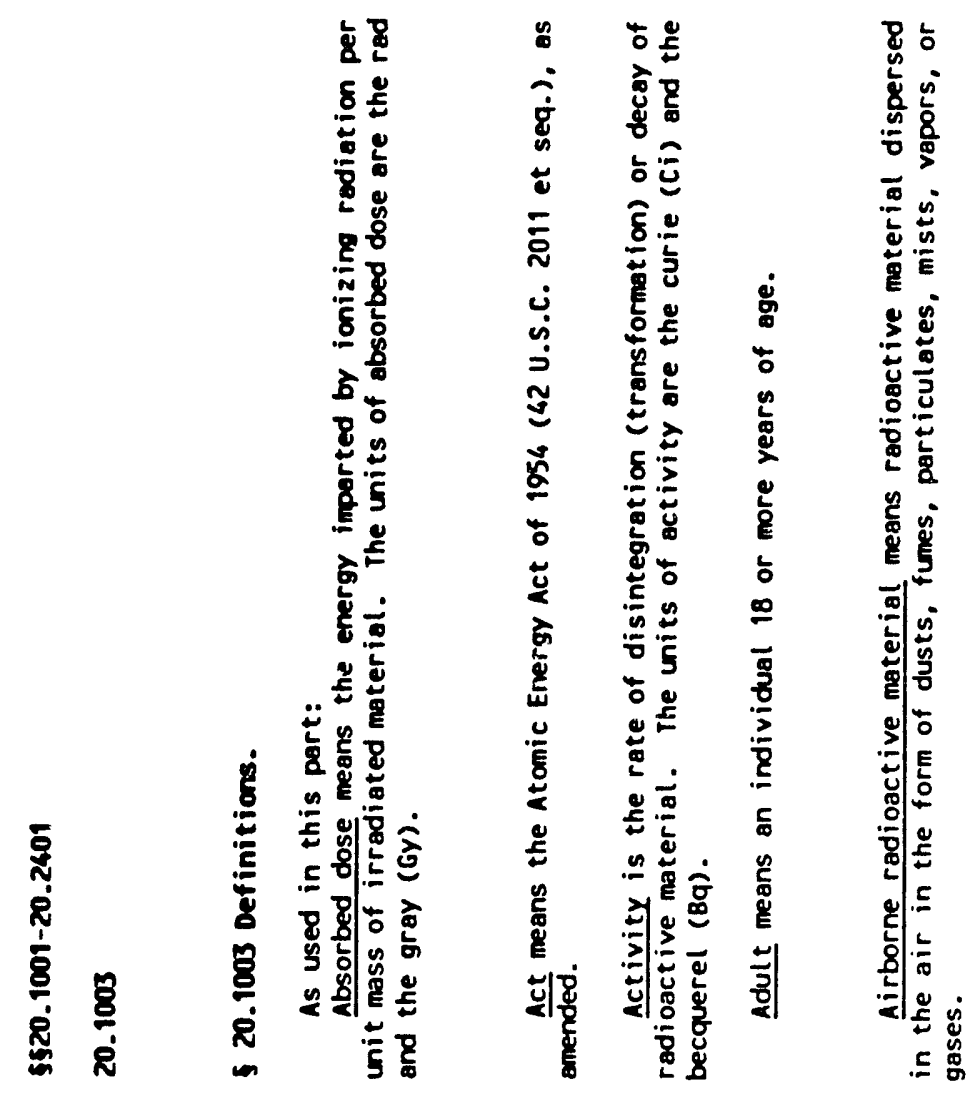

n
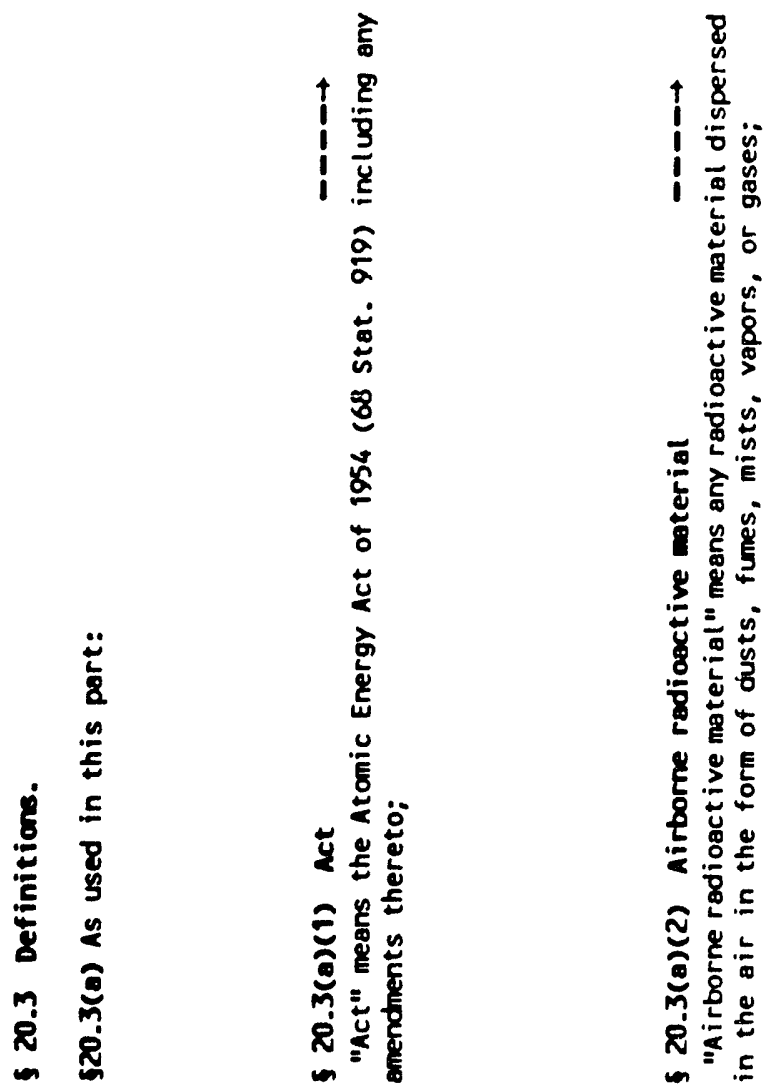

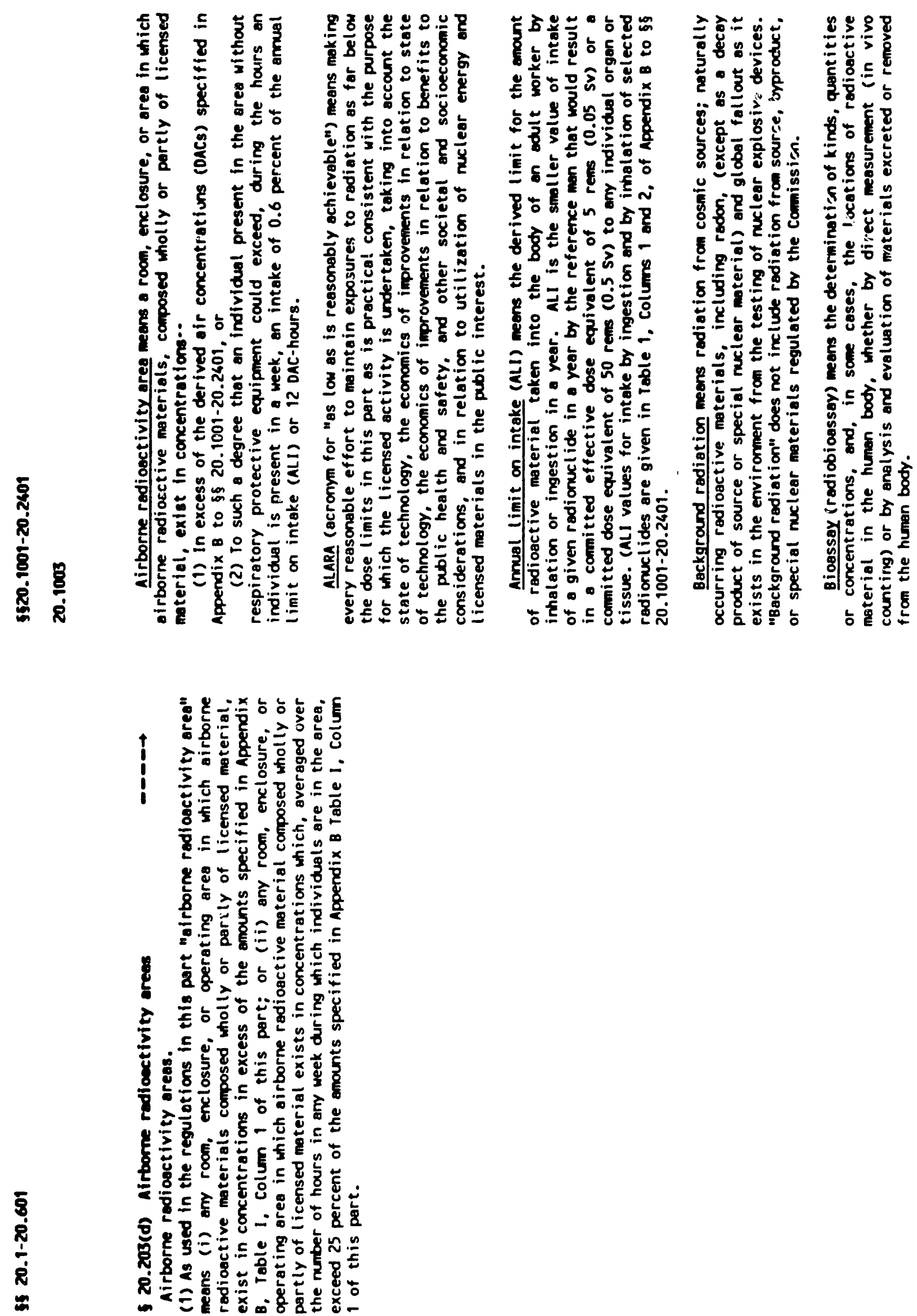

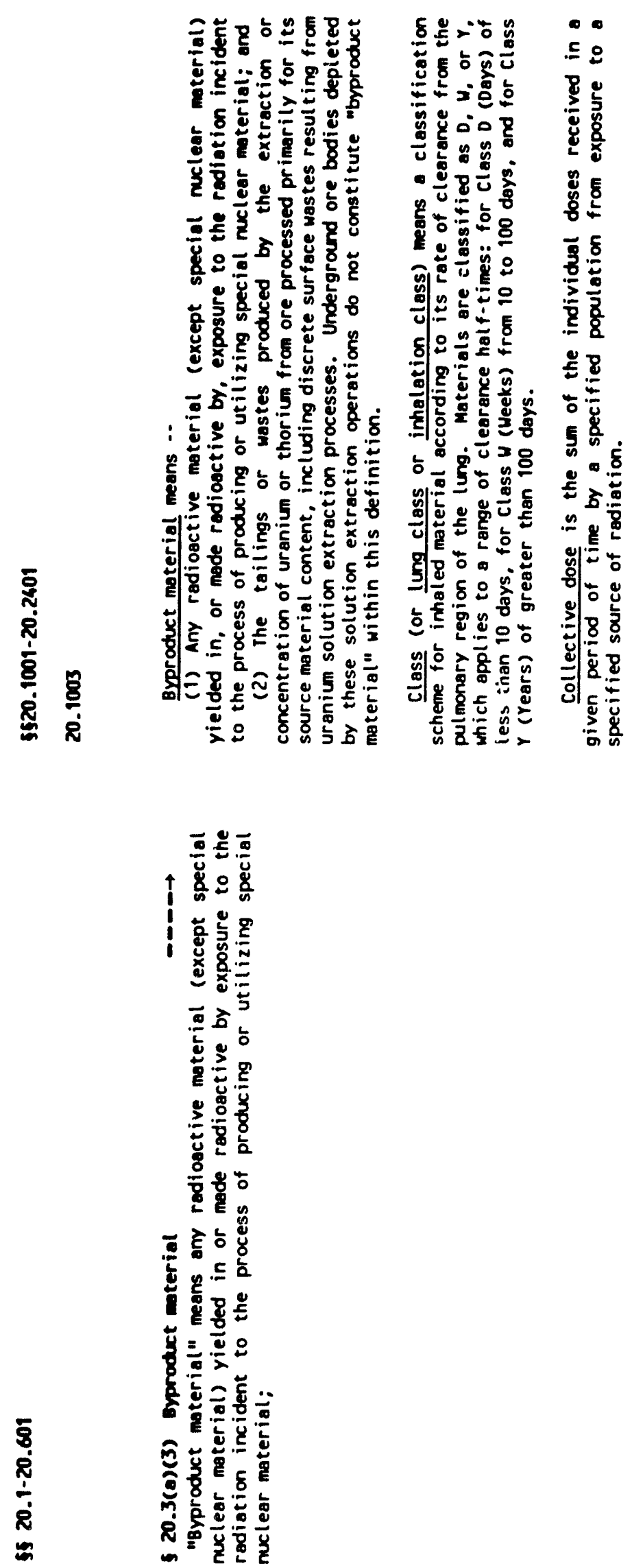


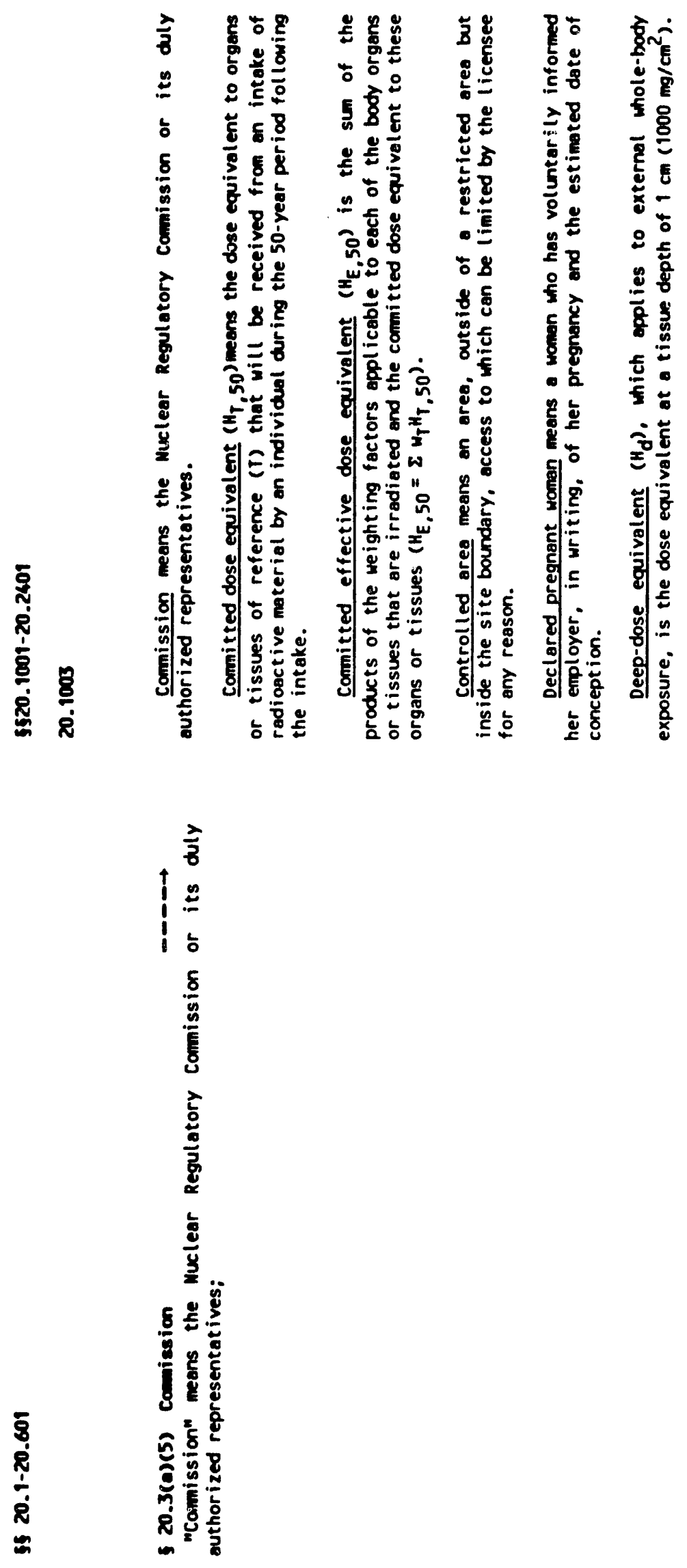




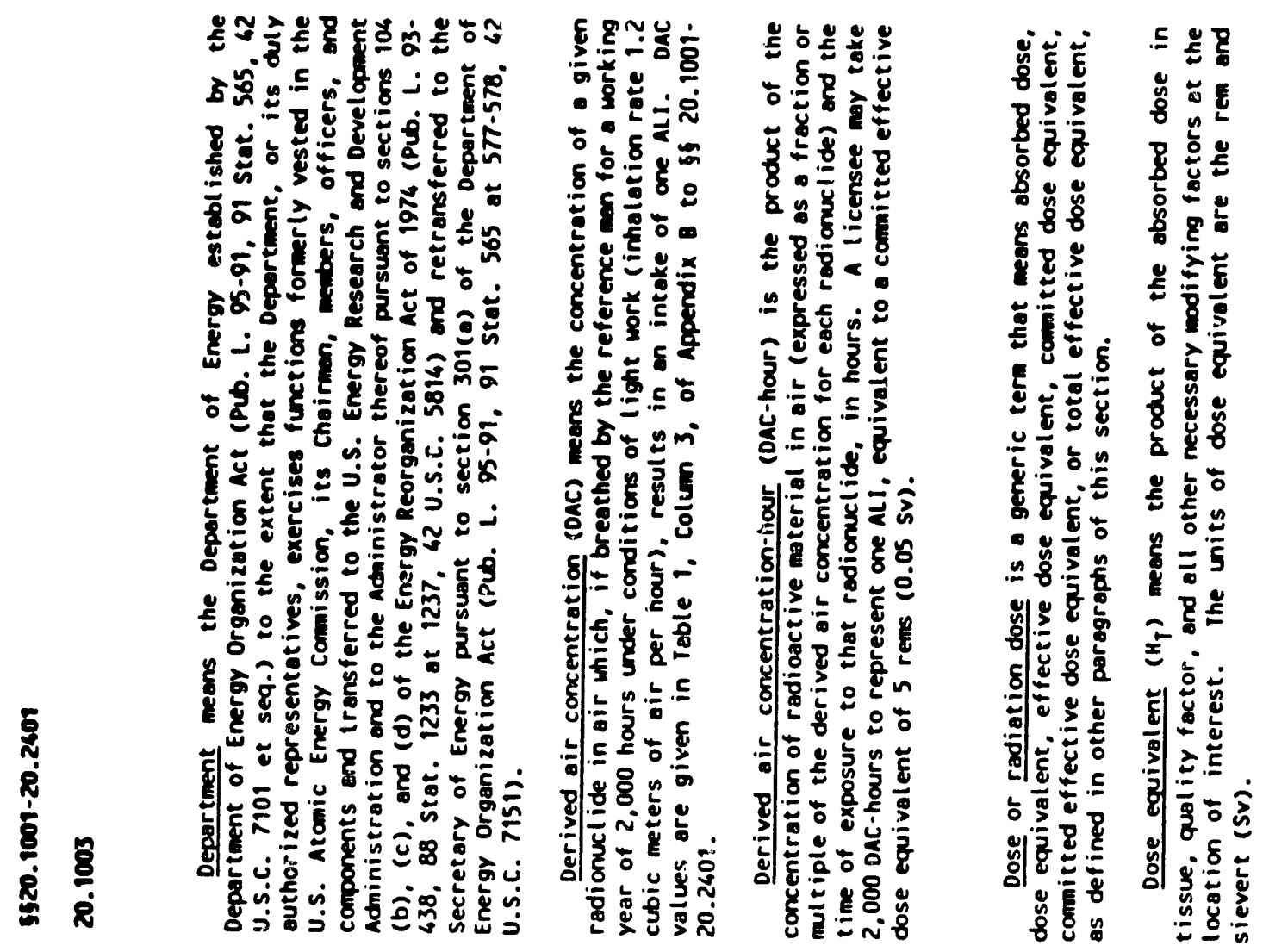

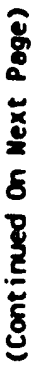

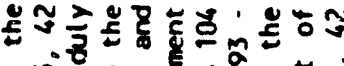

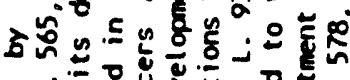

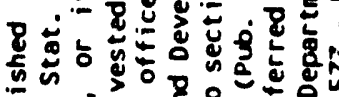

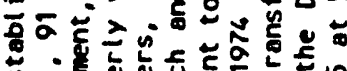

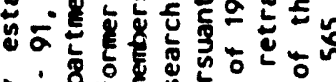

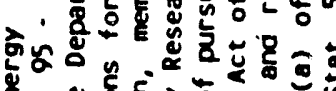

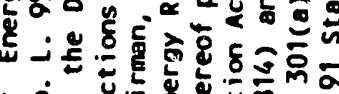

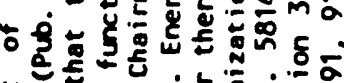

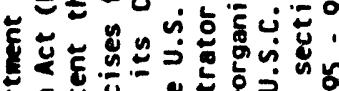

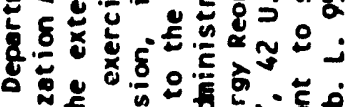

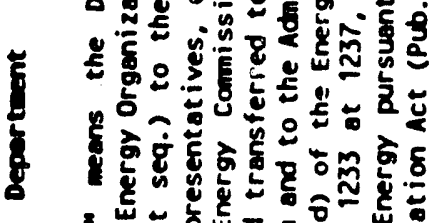

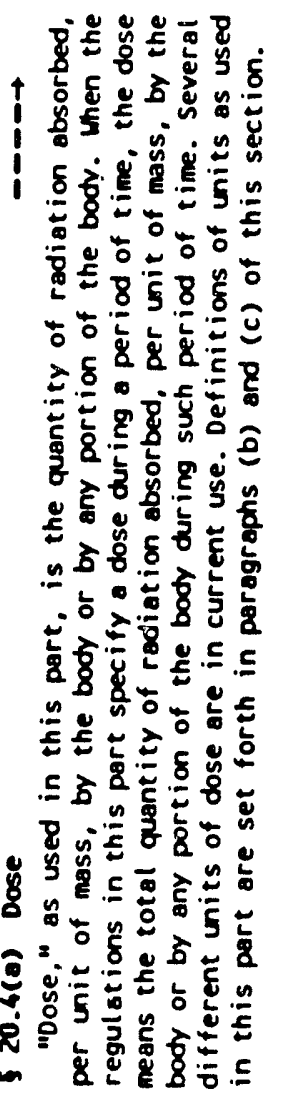



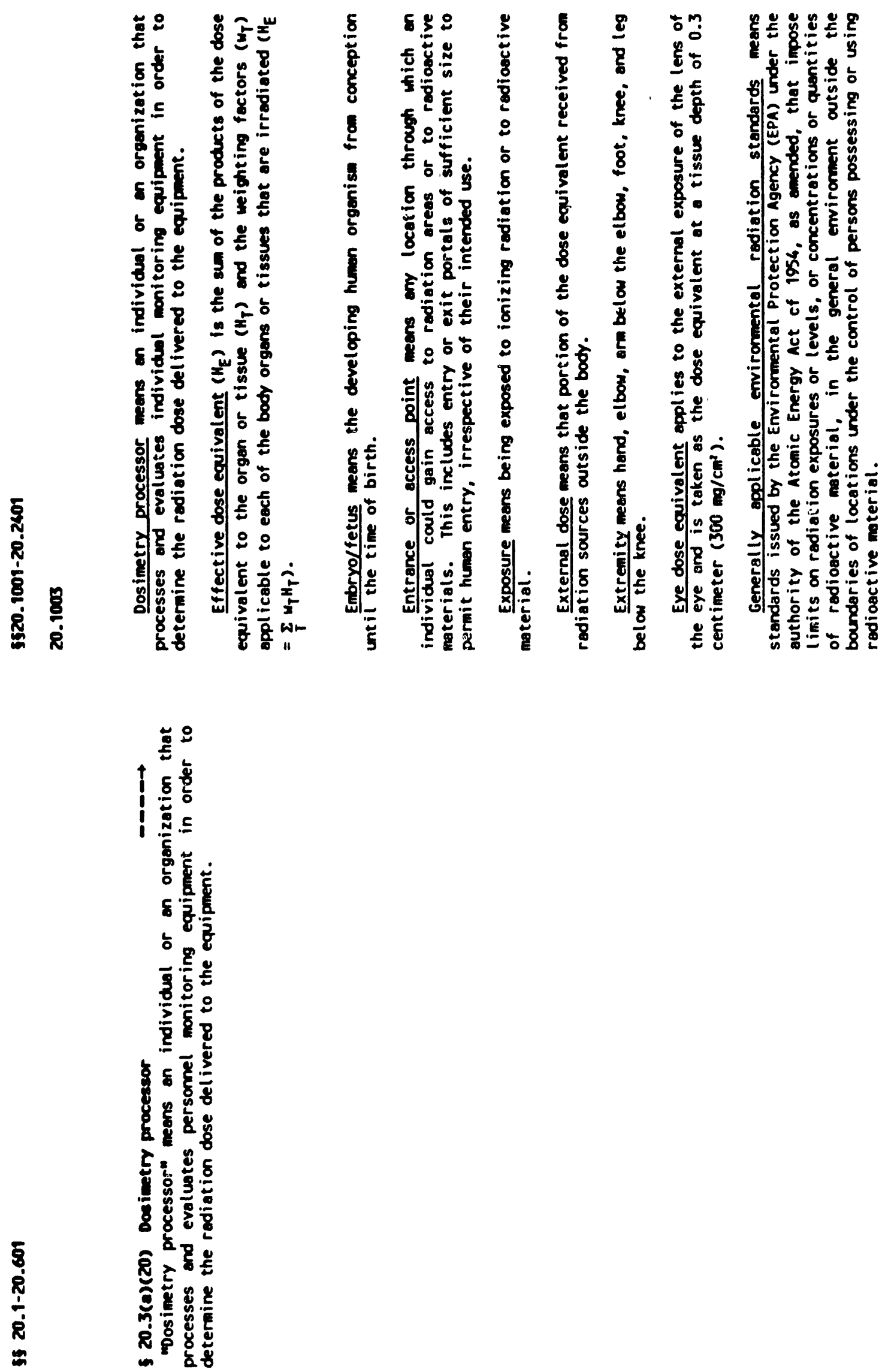

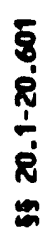




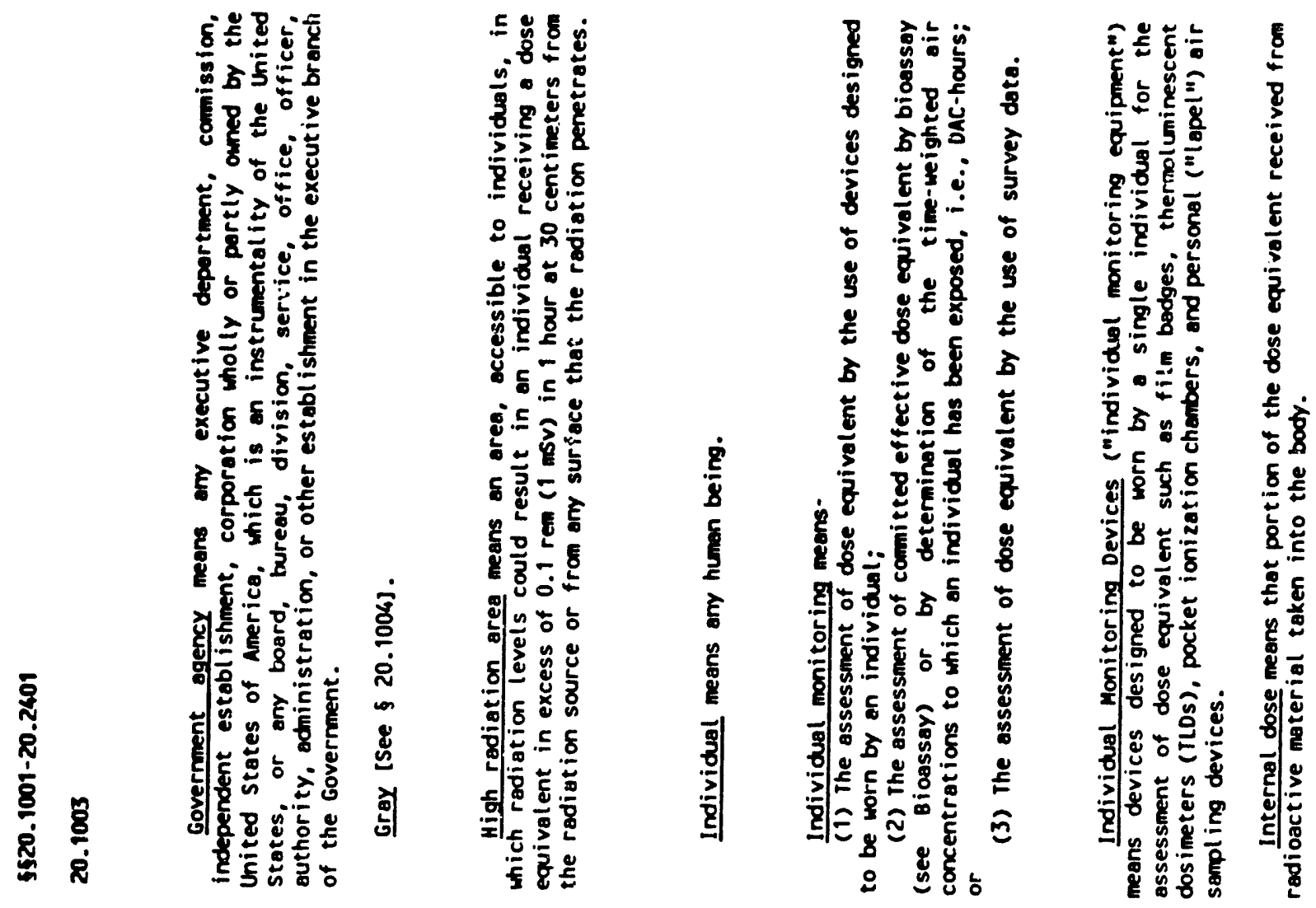

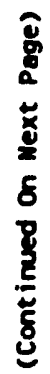
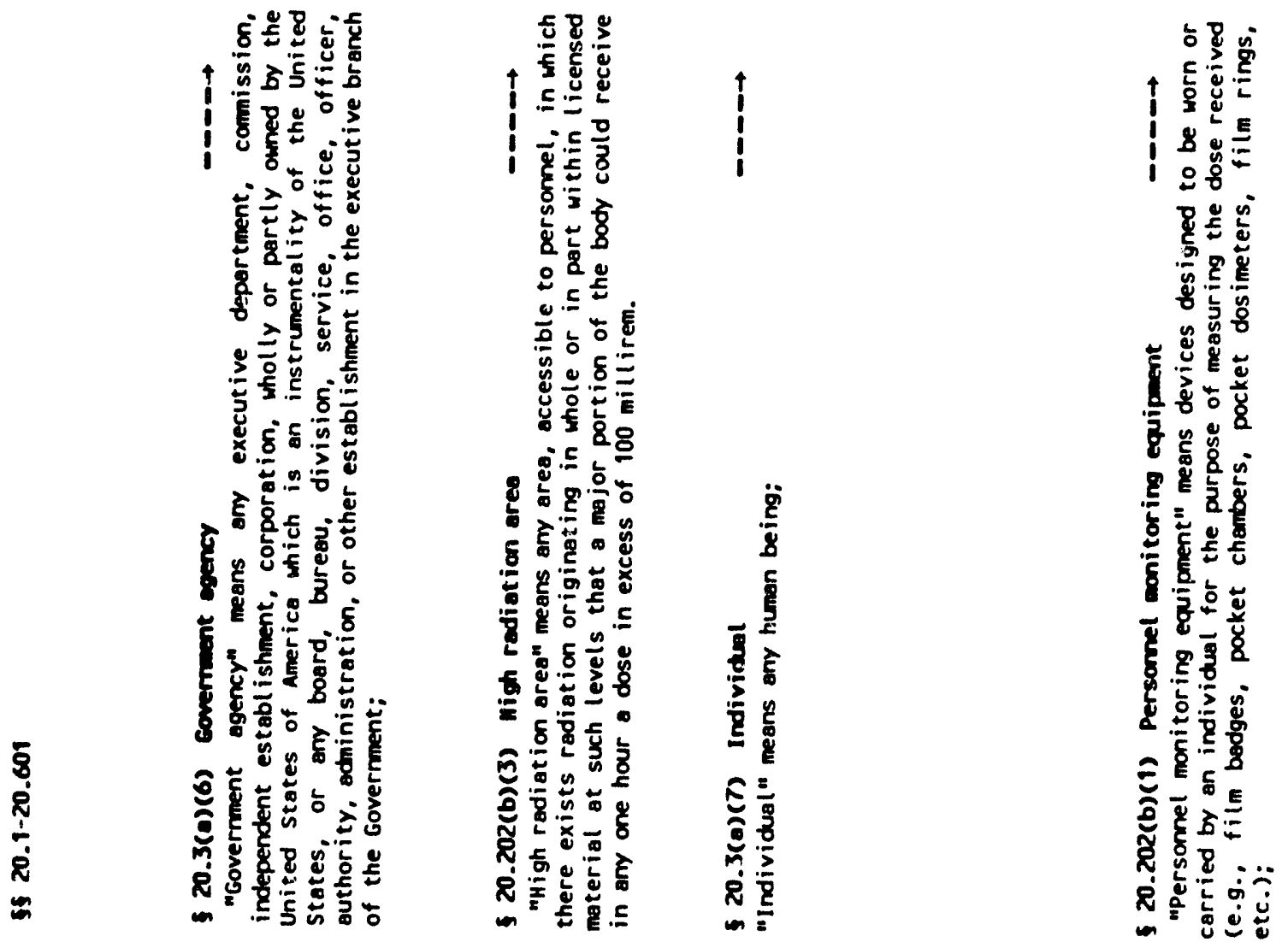


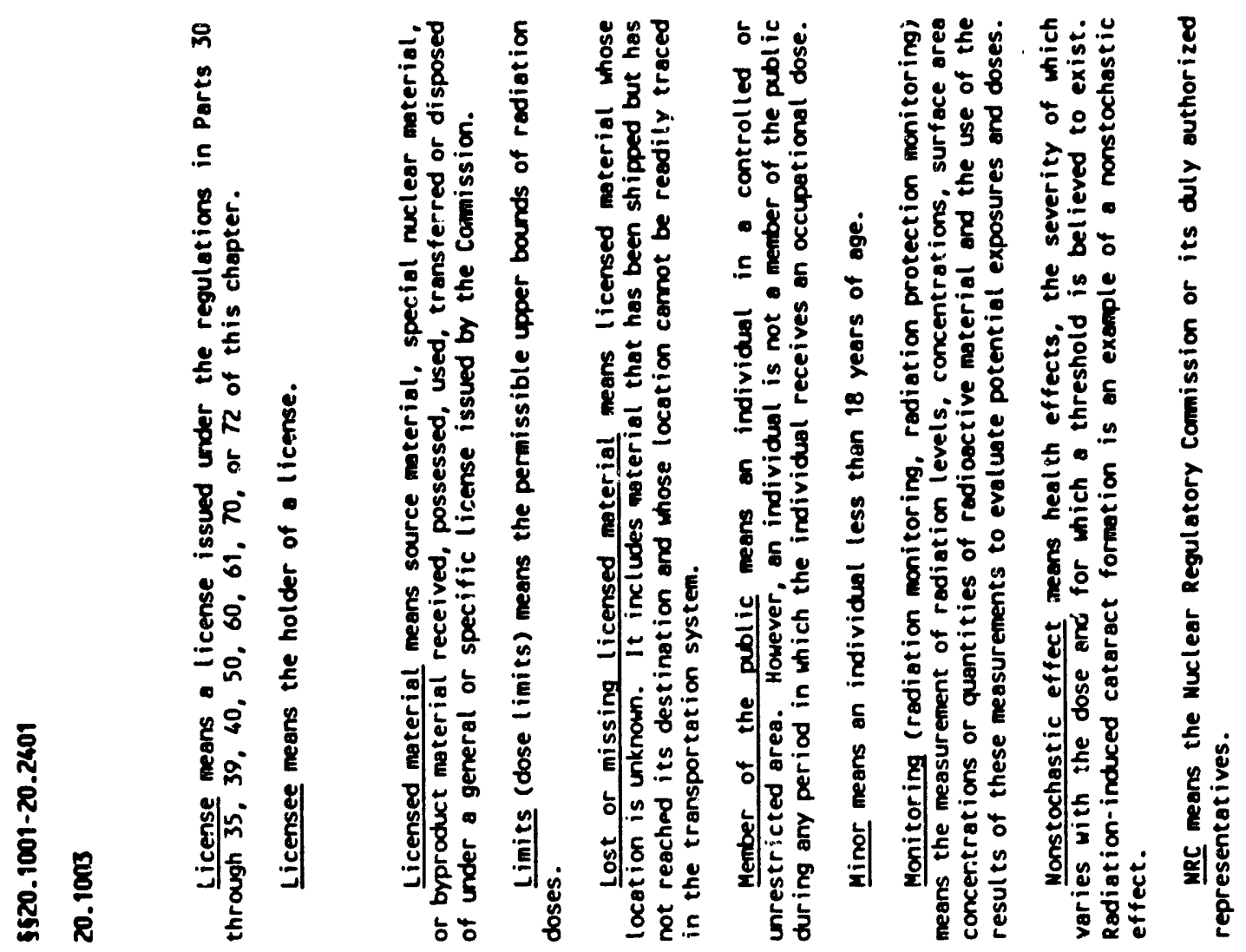

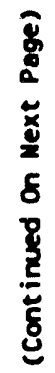

$\simeq$

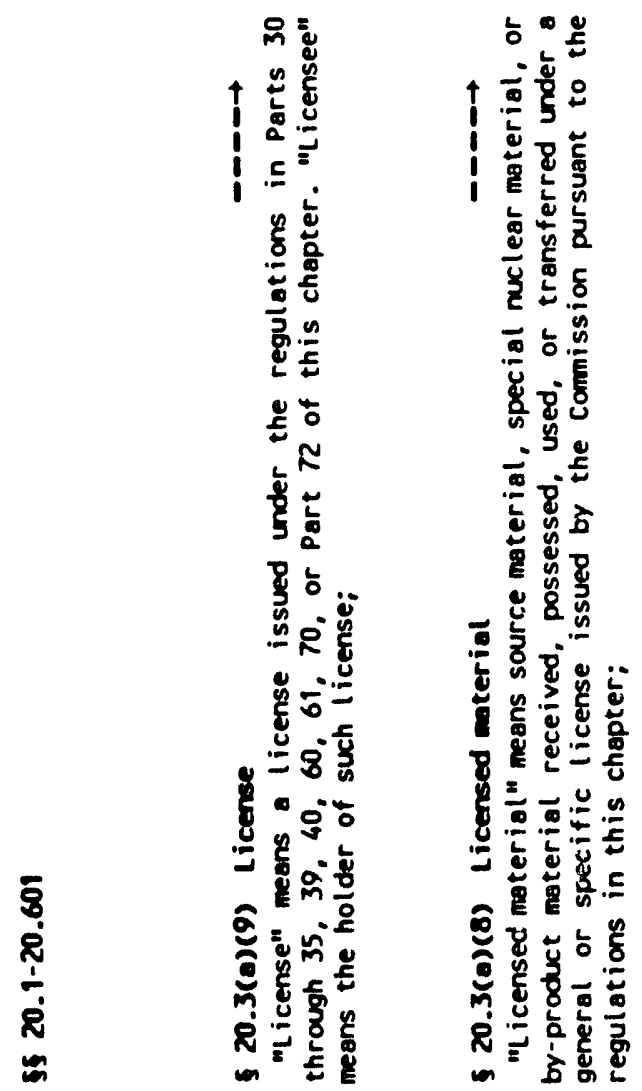



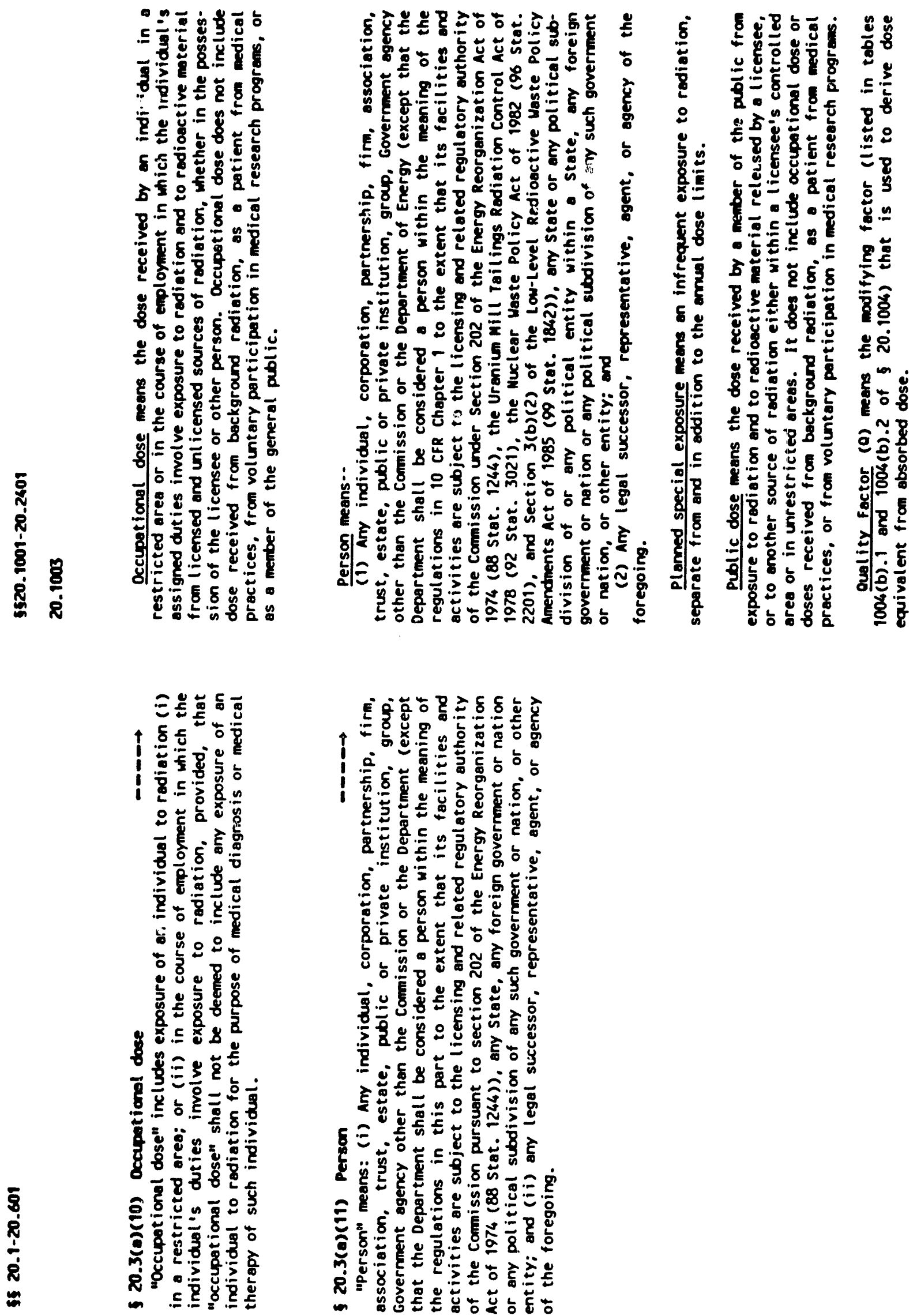

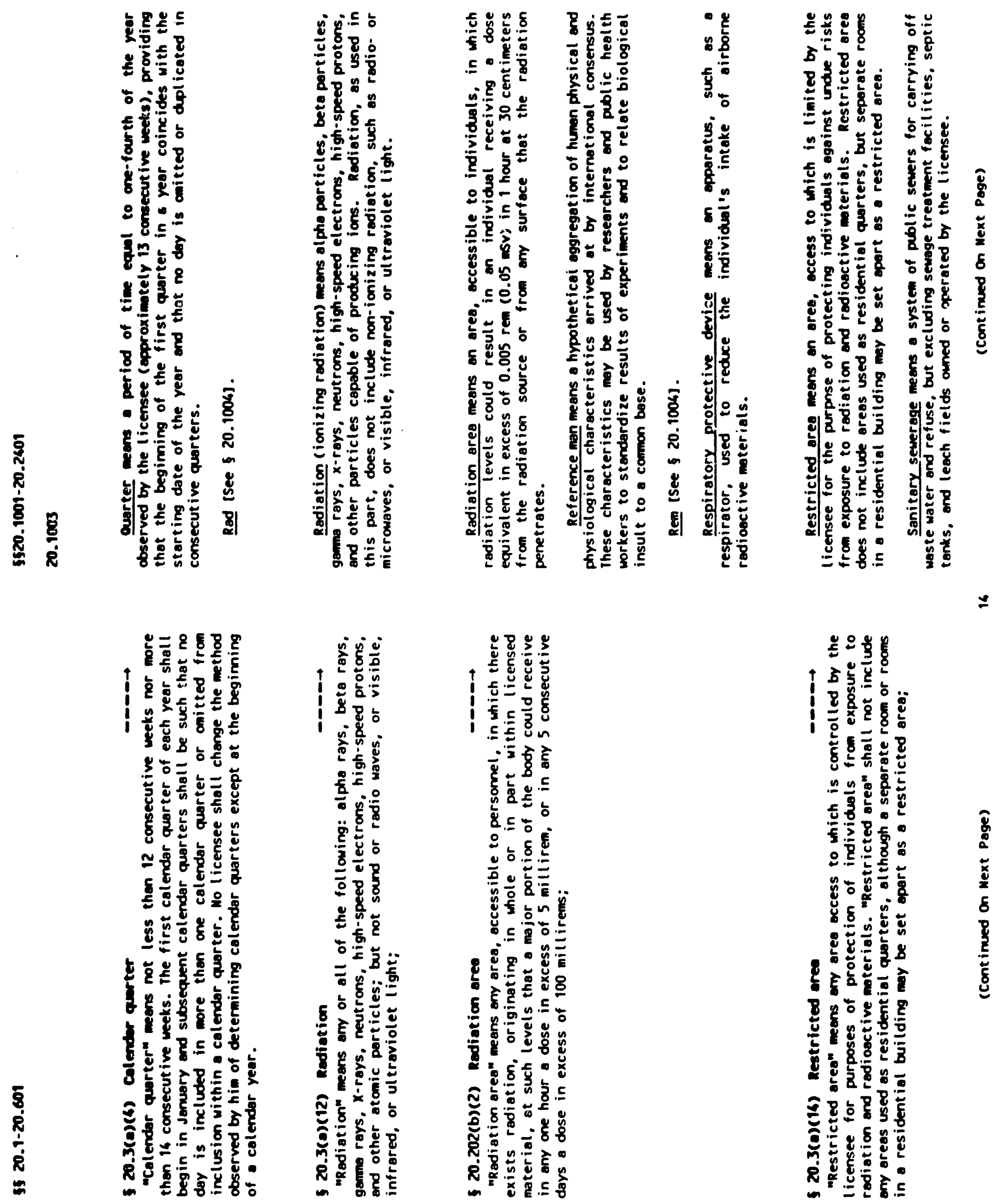

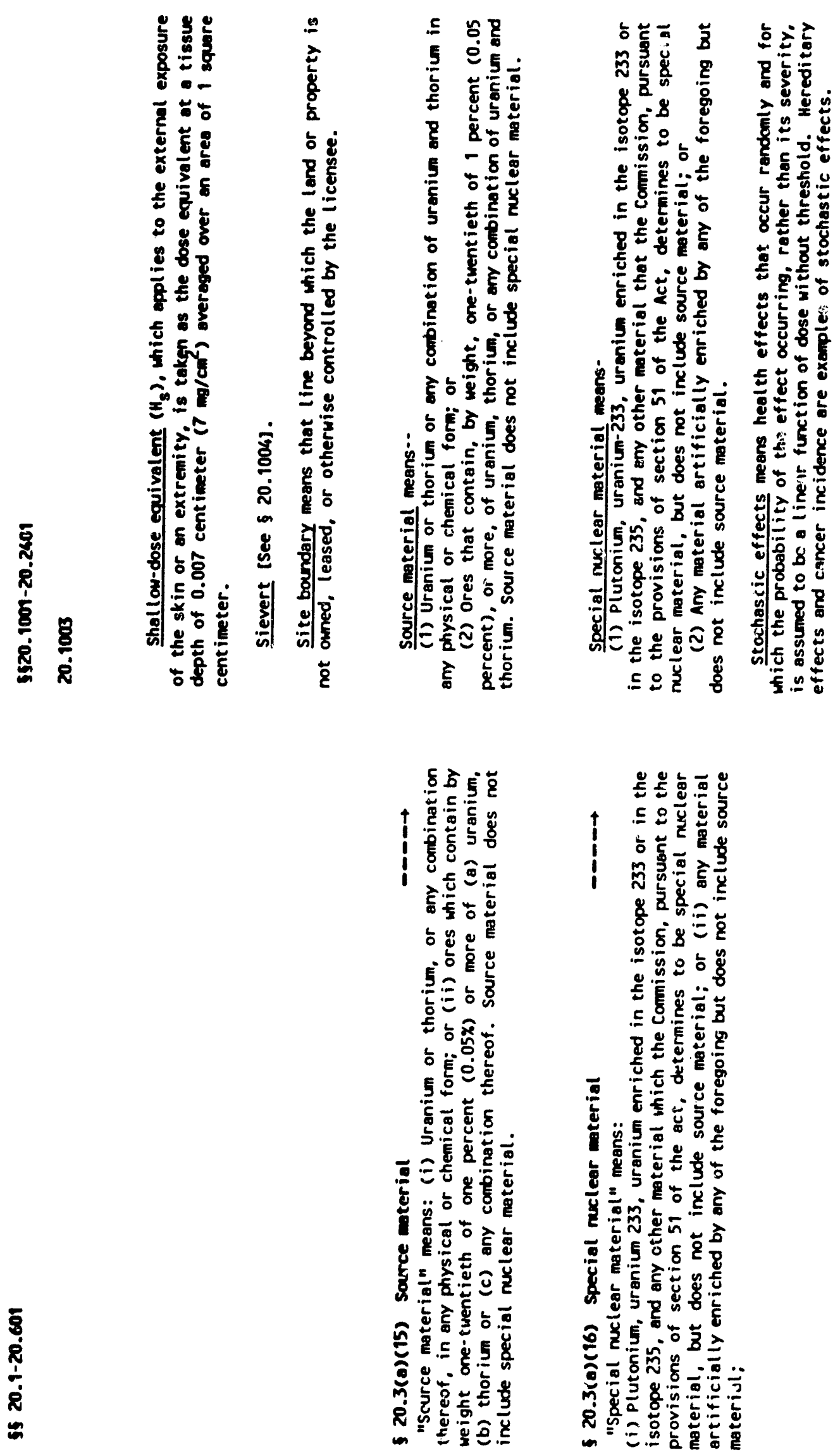

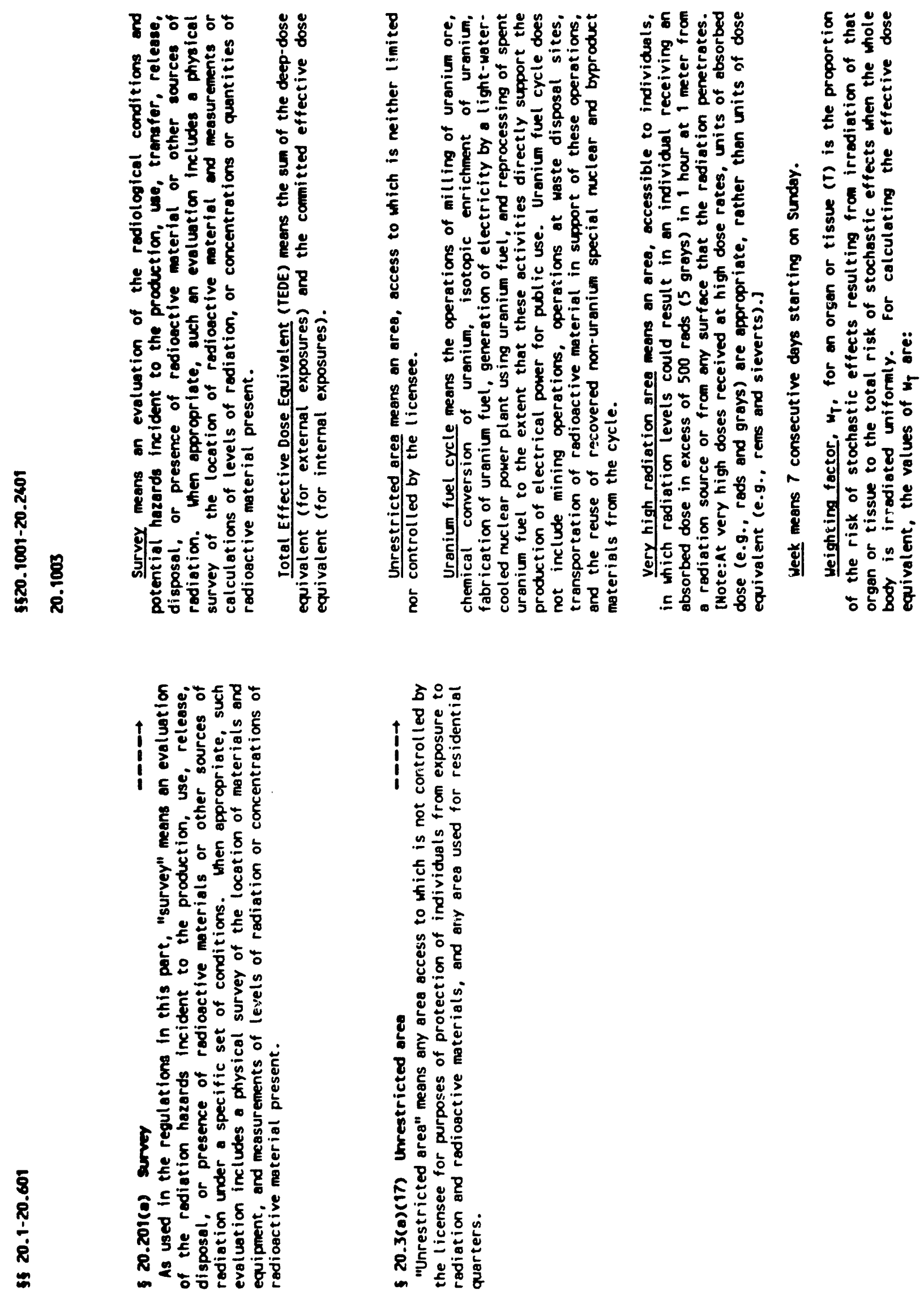


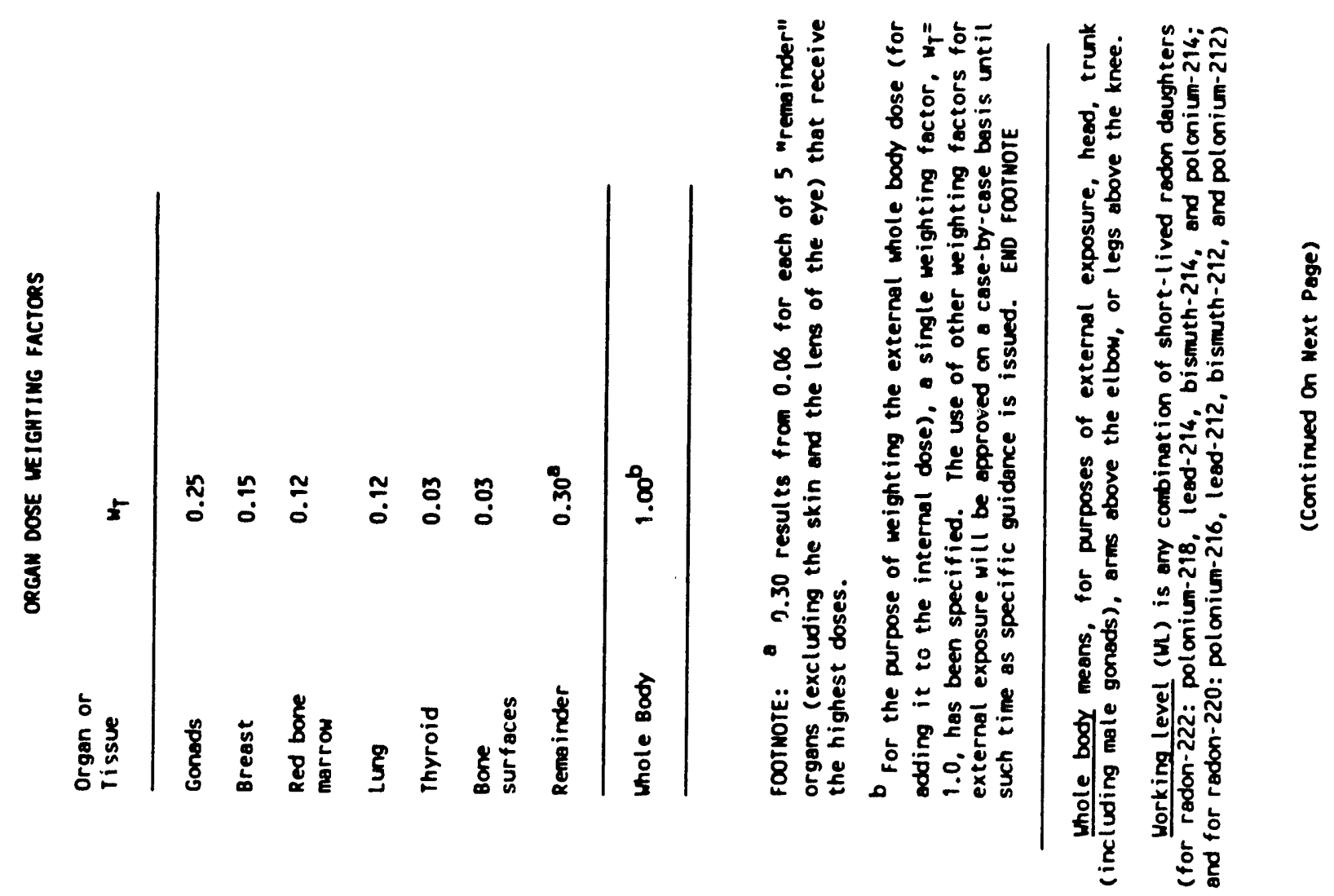

$=$ 

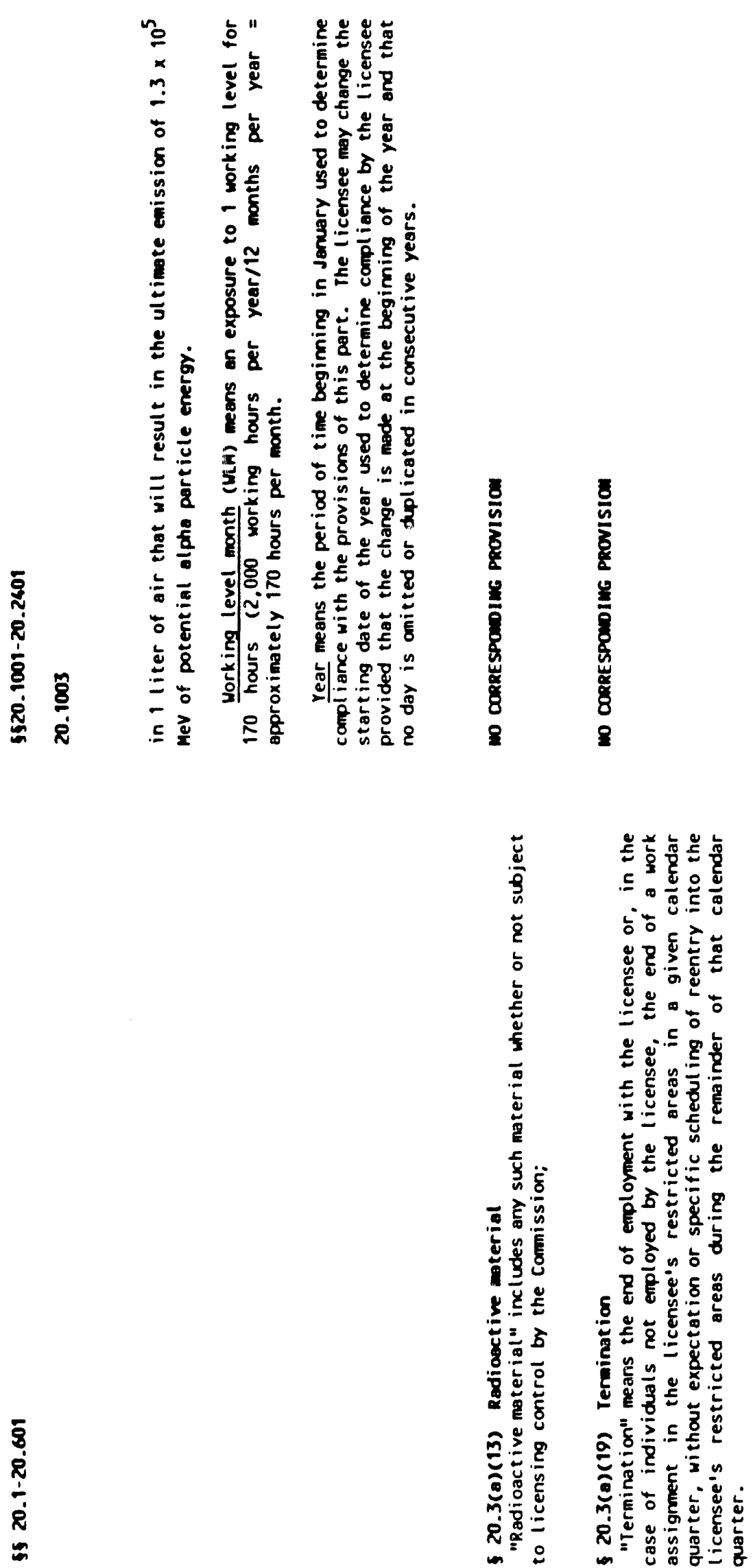

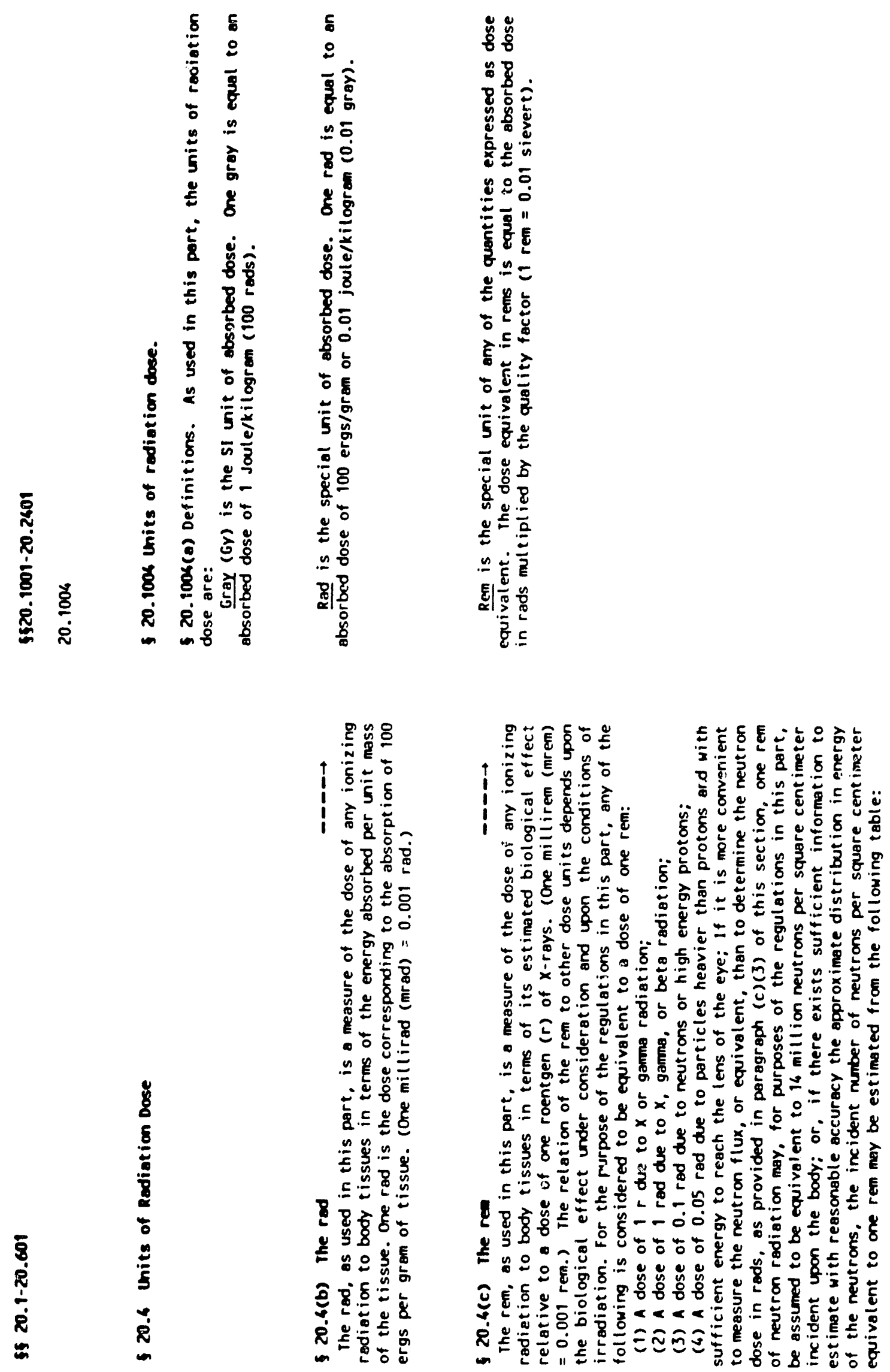

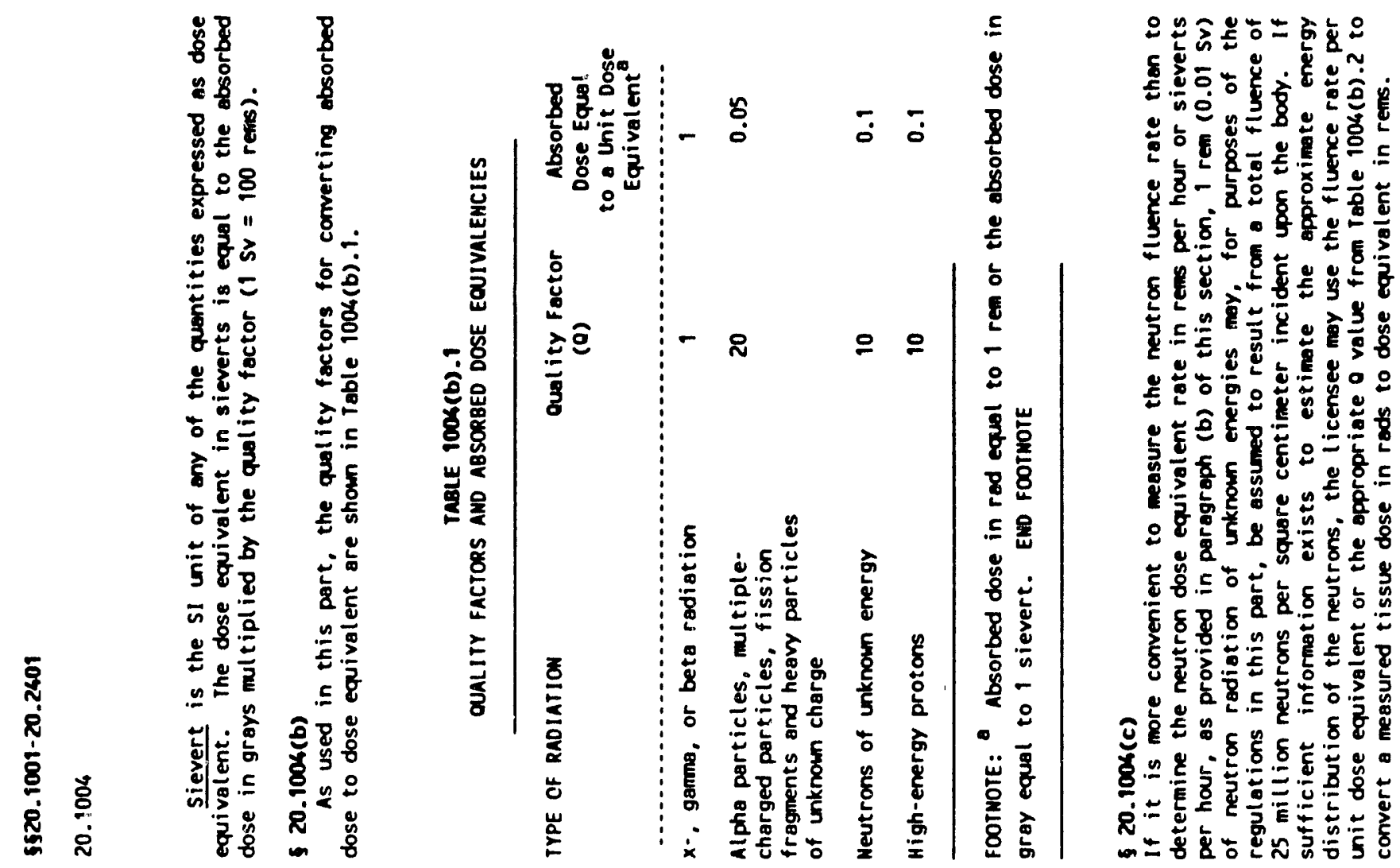


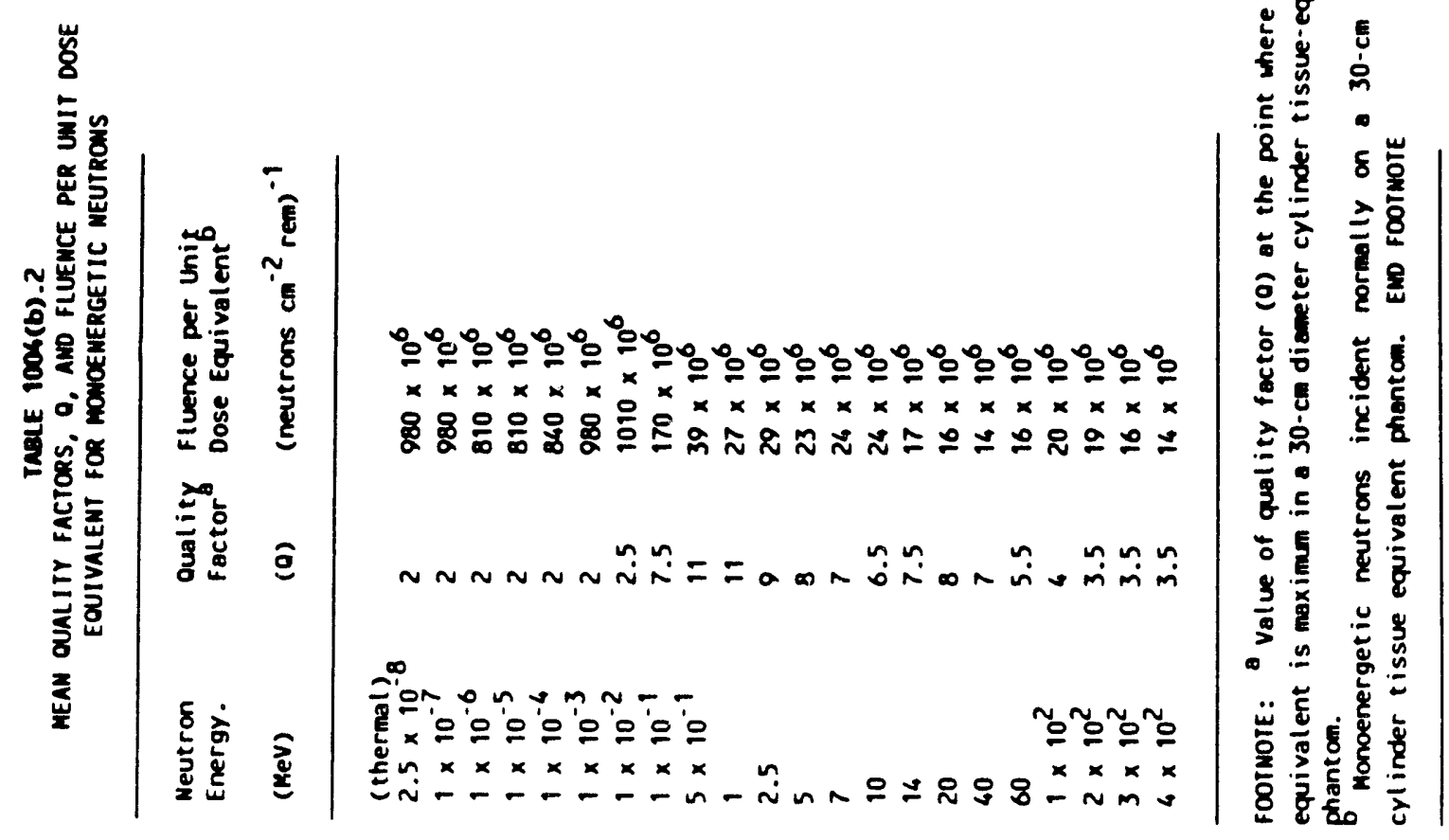



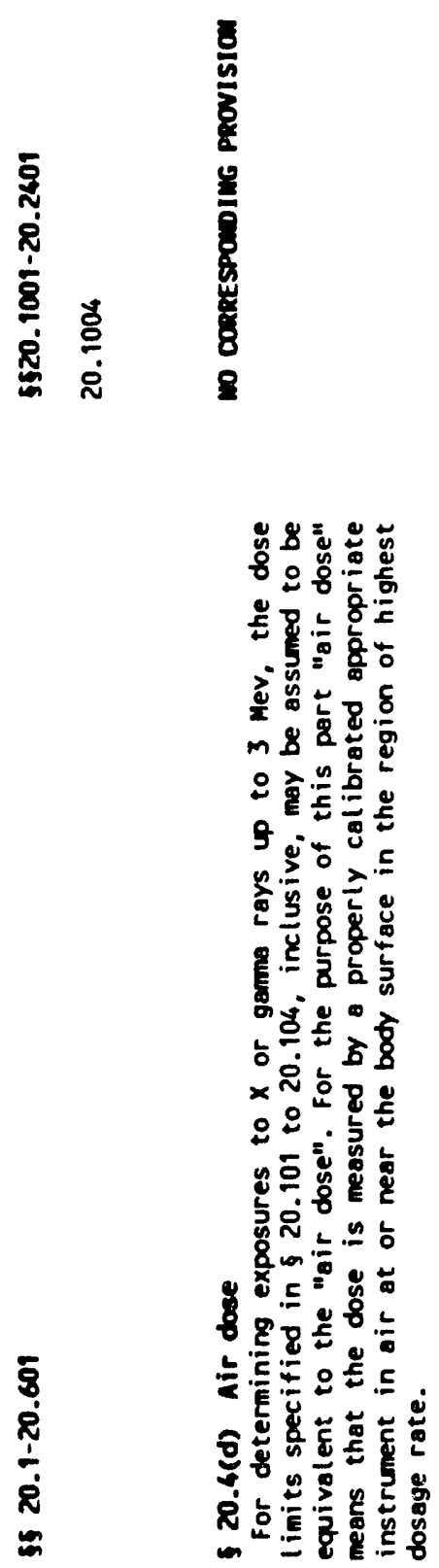

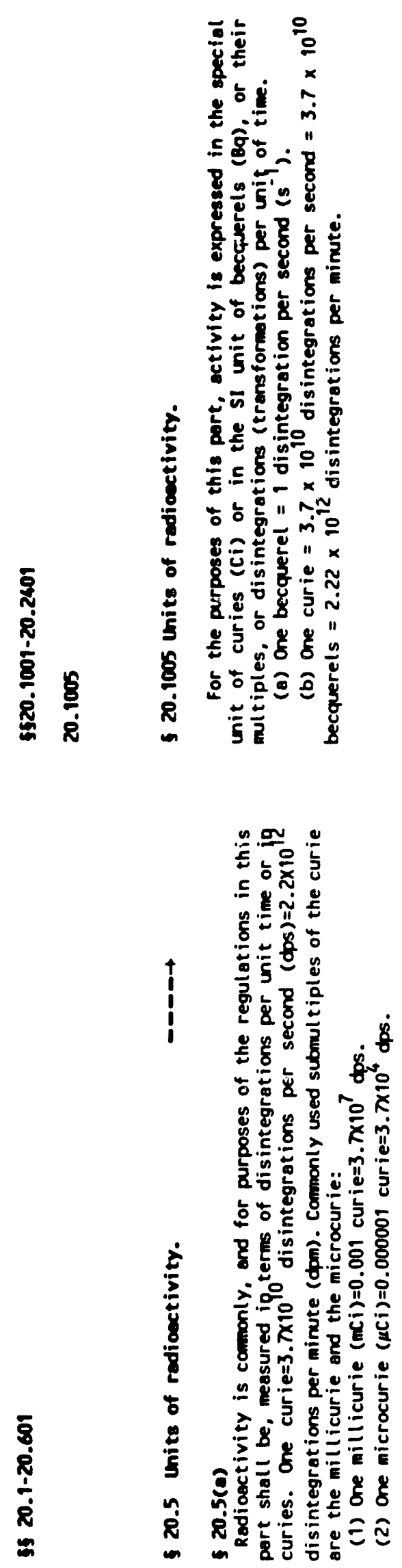


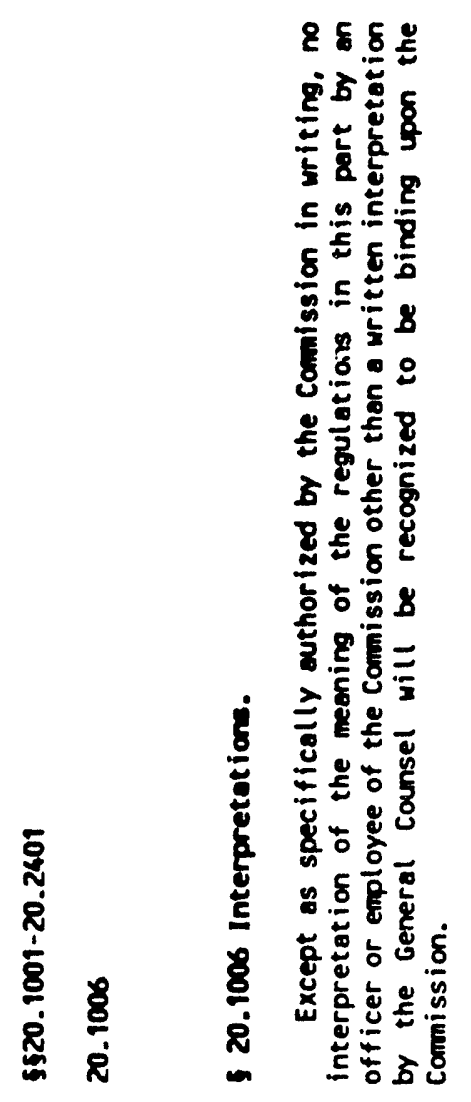

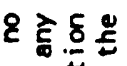

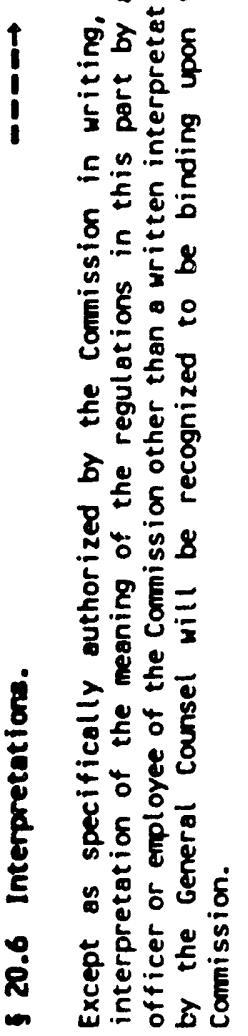



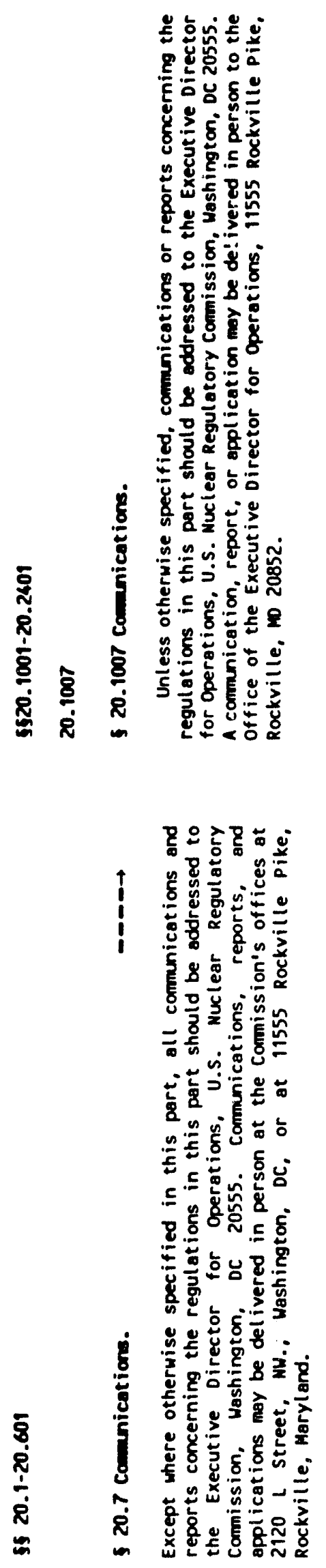


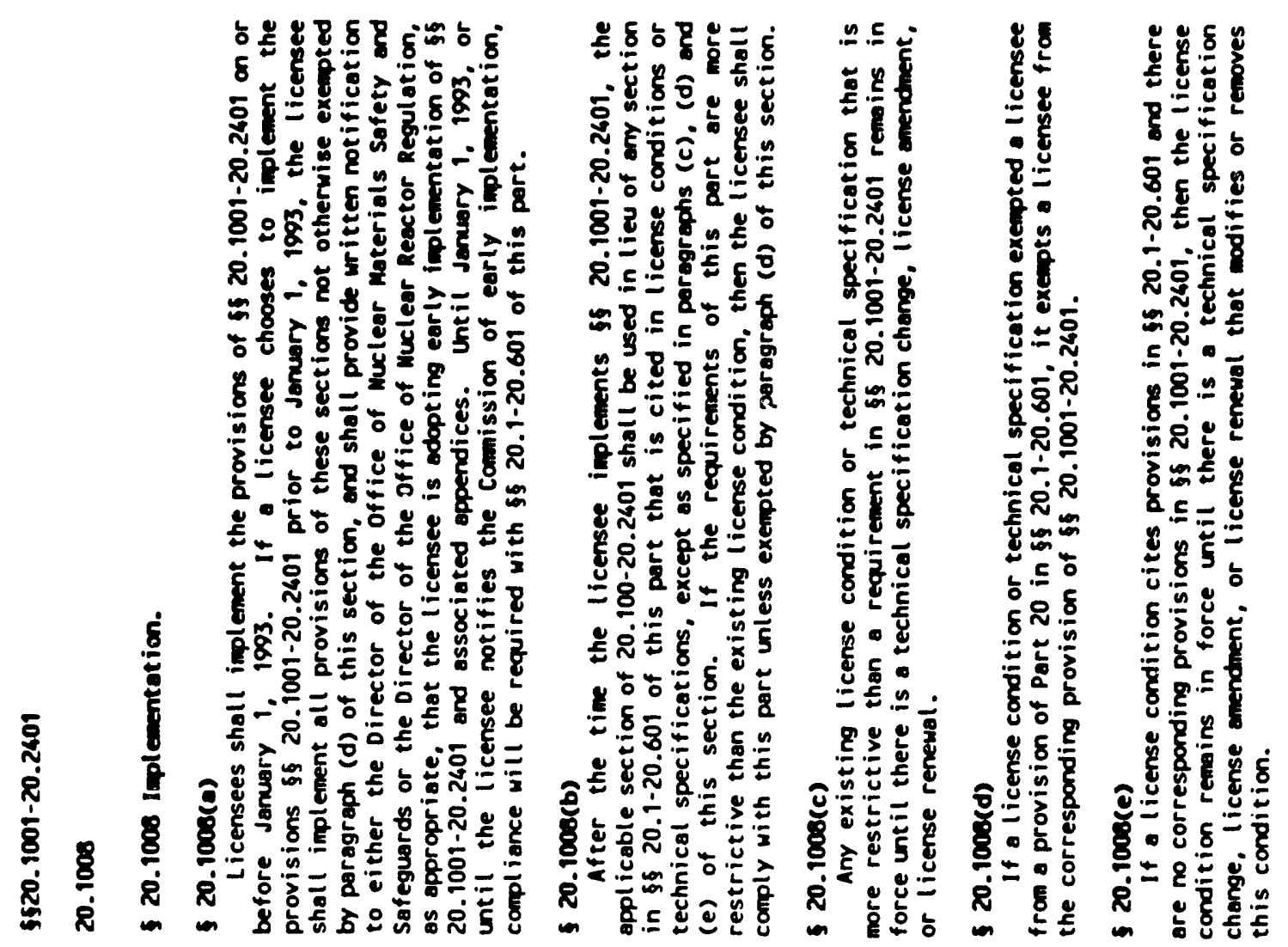




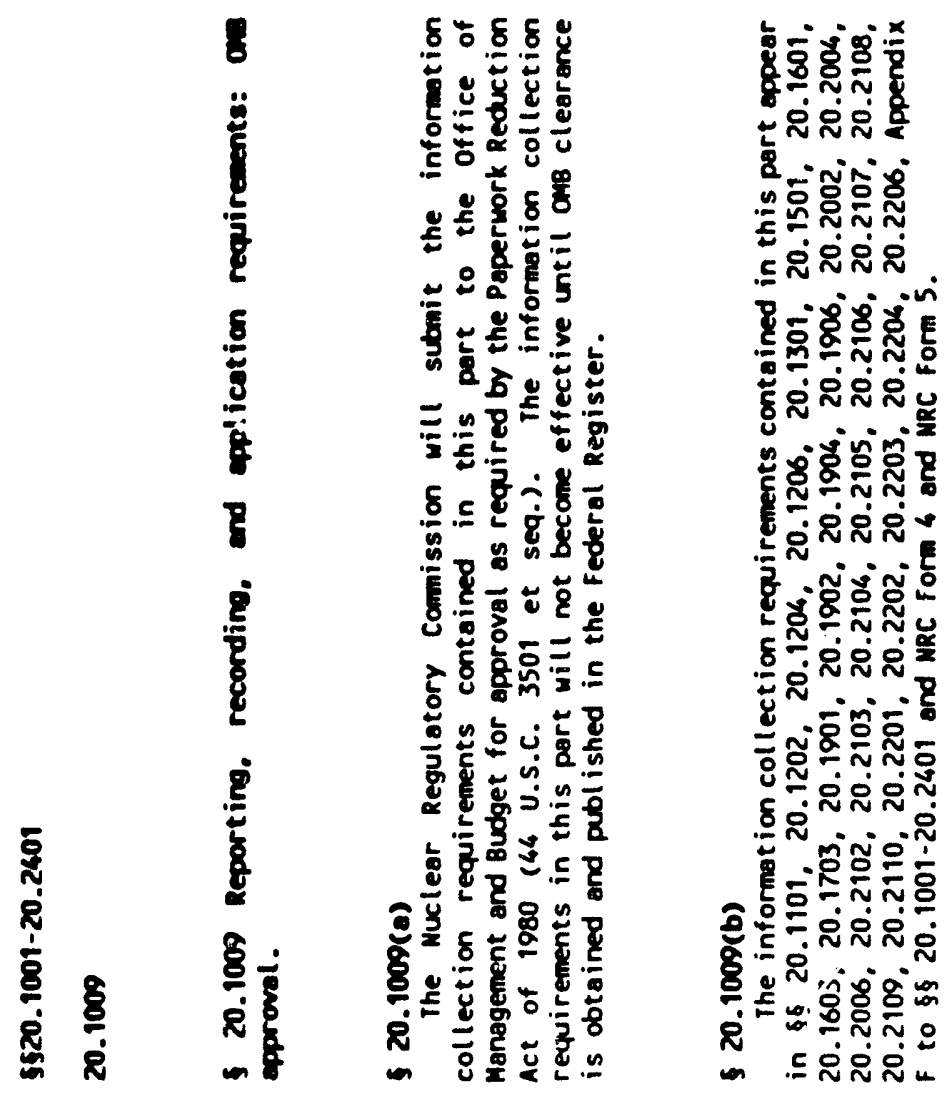

$\hat{\sim}$

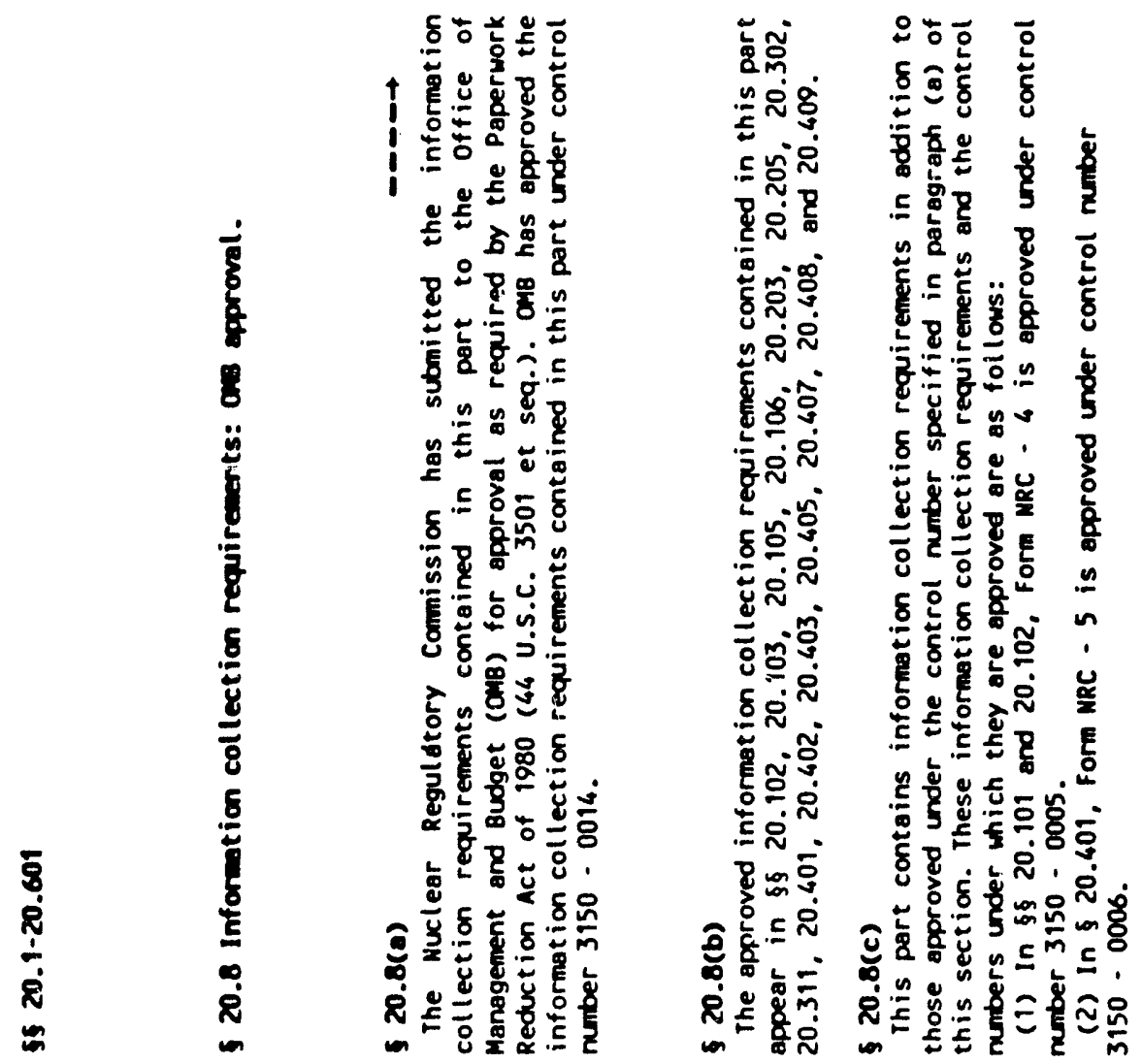




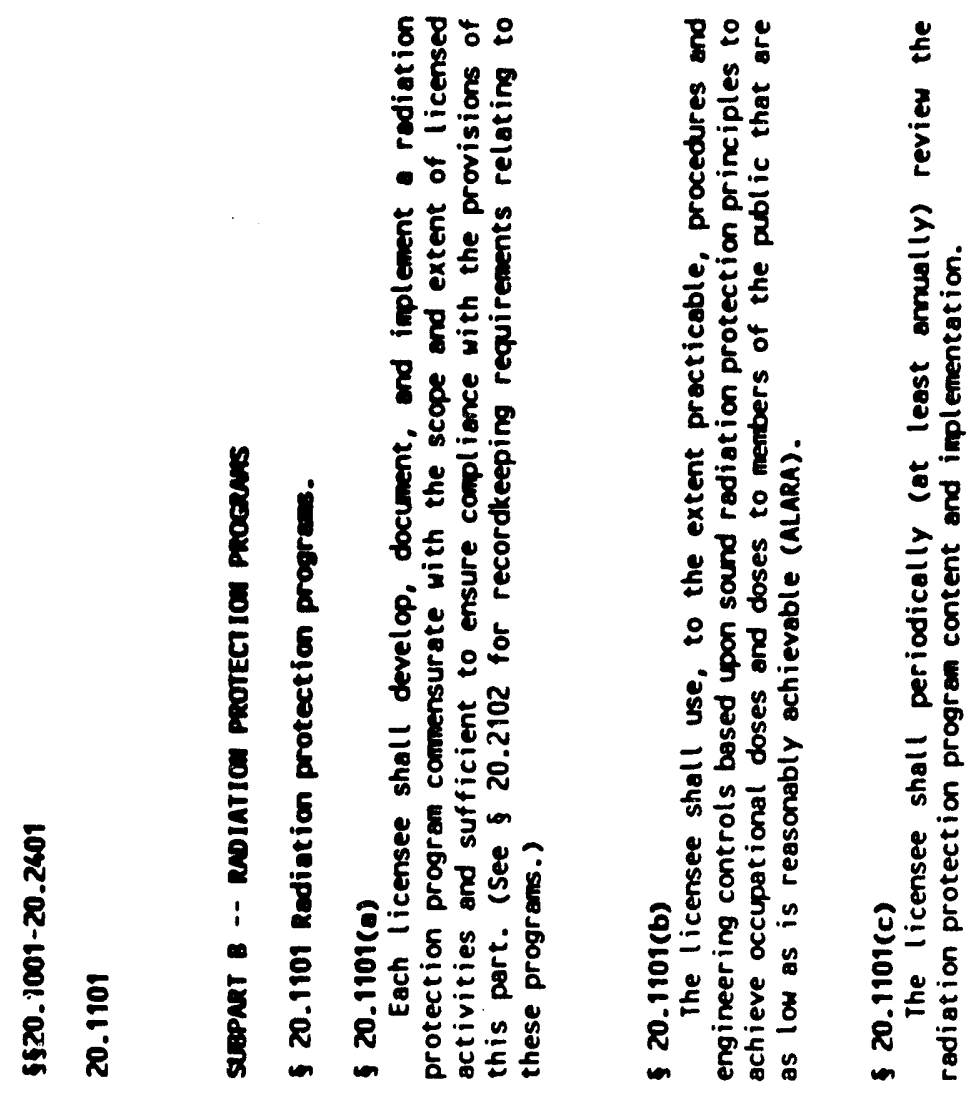

జ

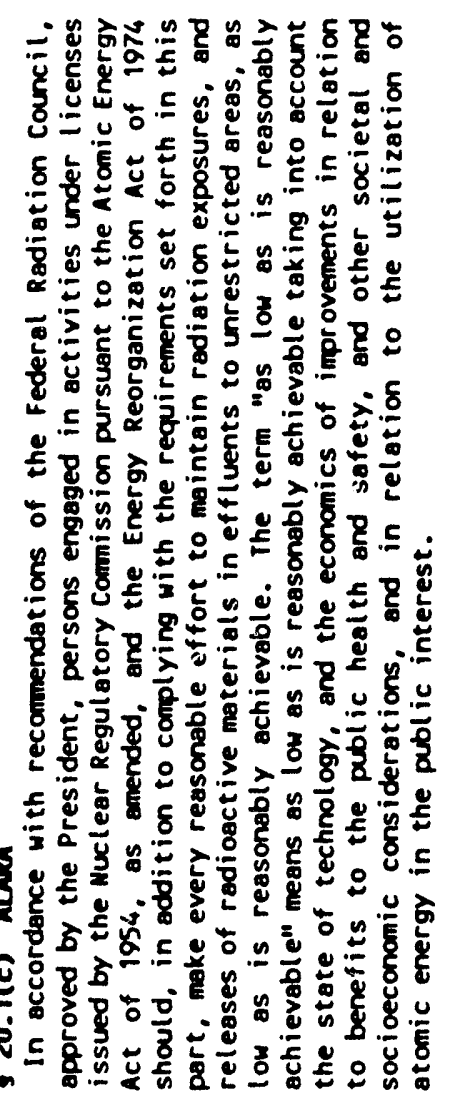



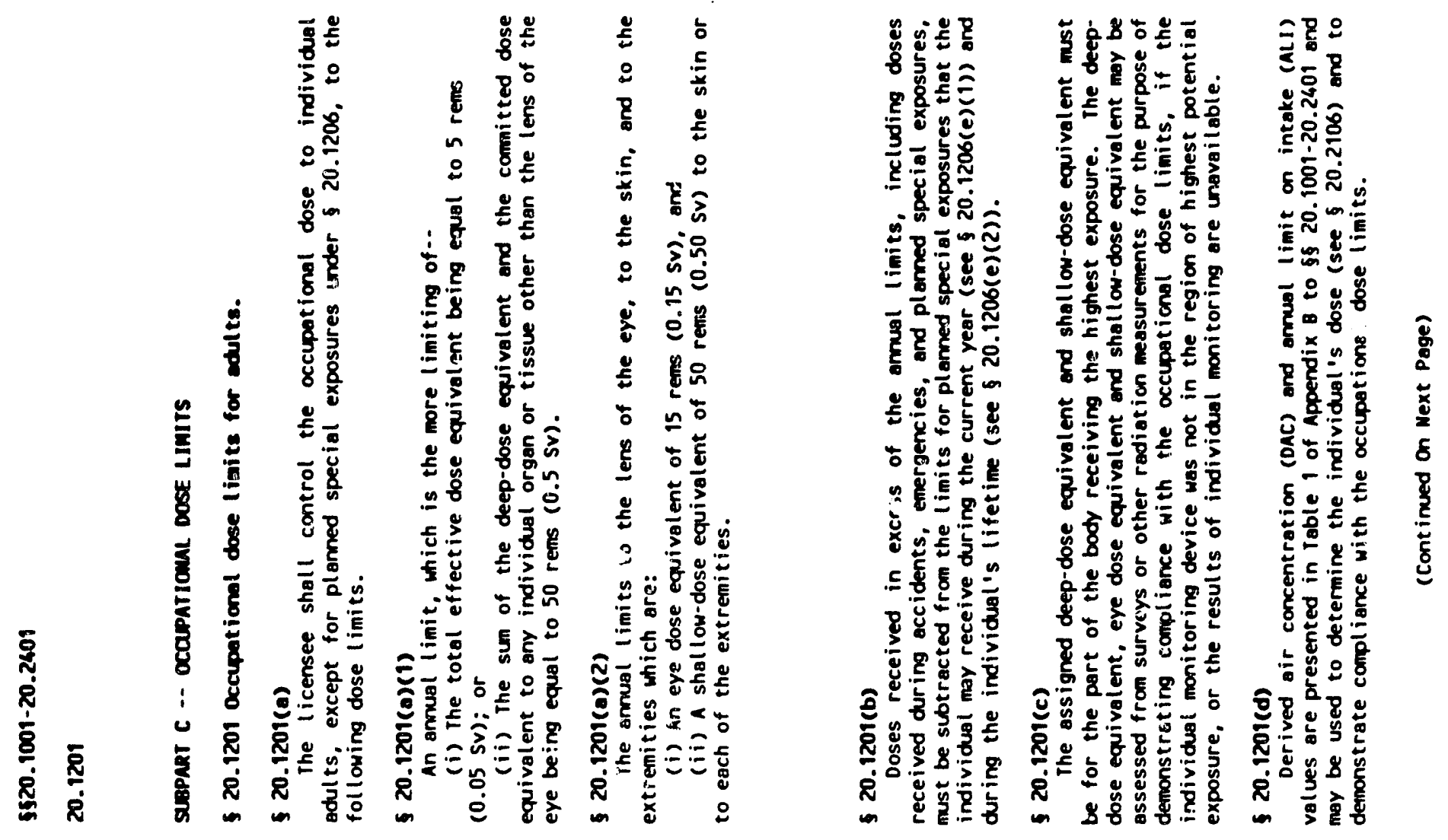

2
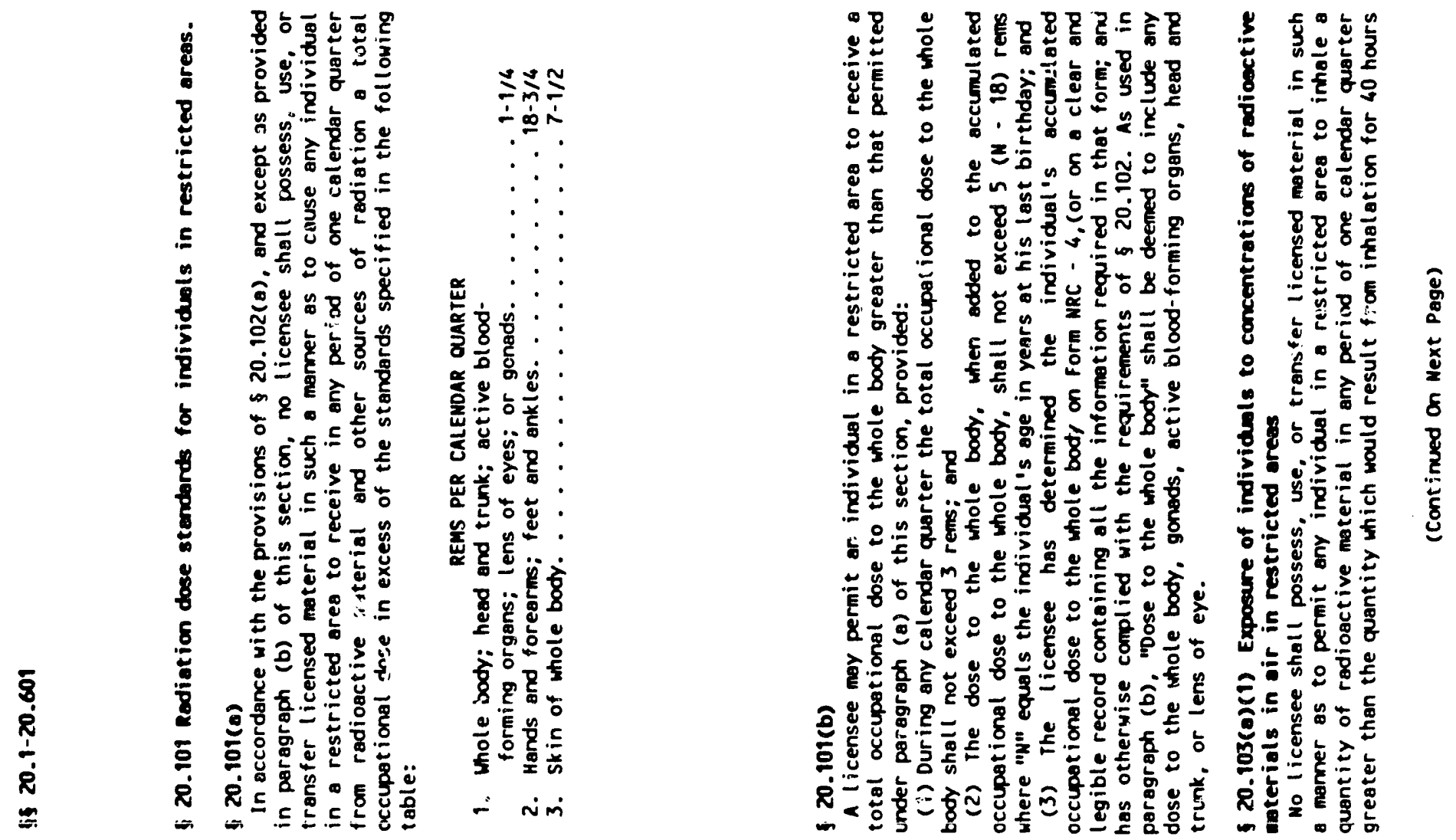


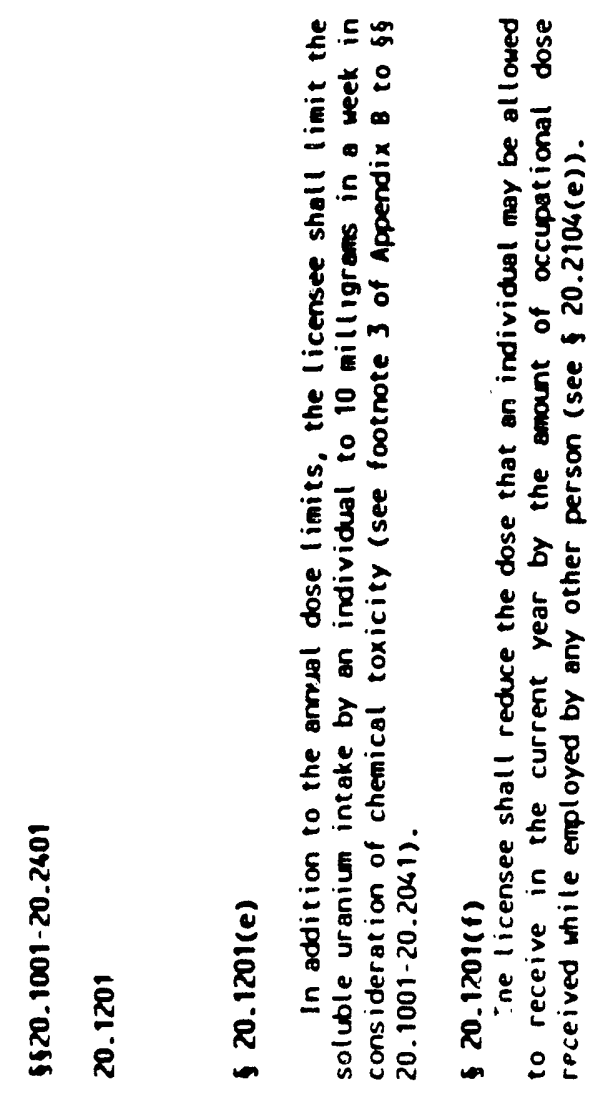

요

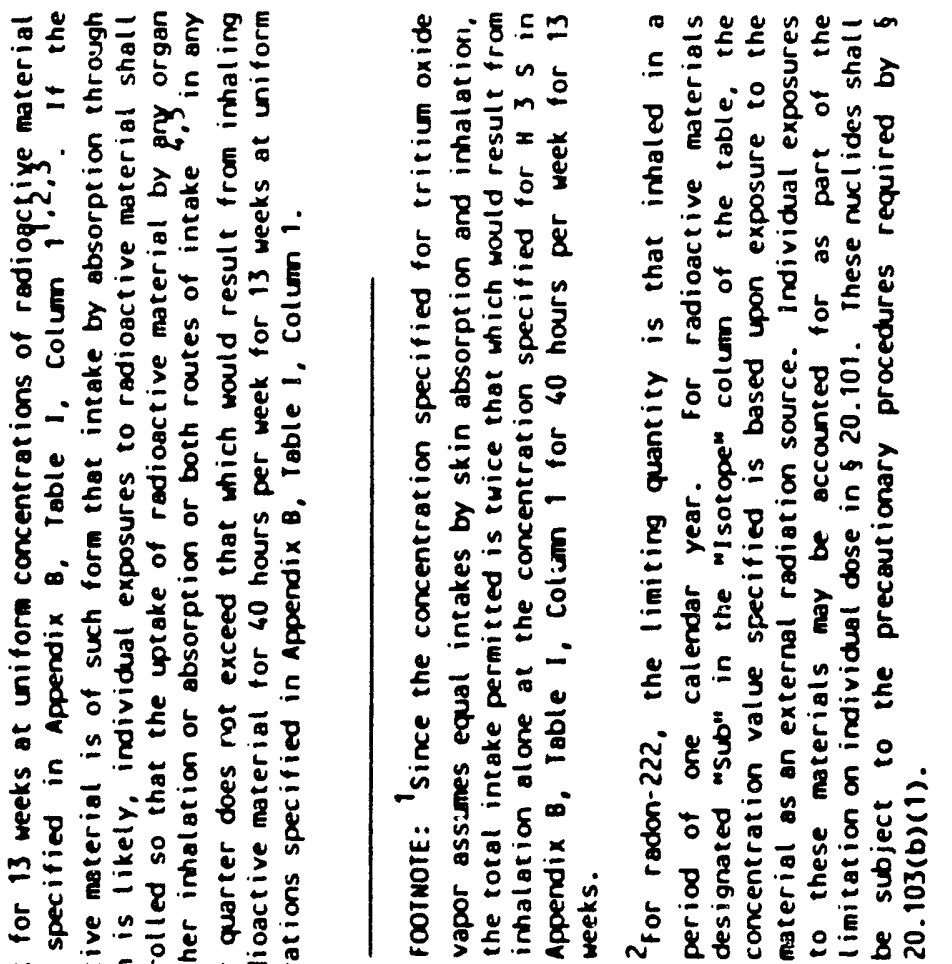

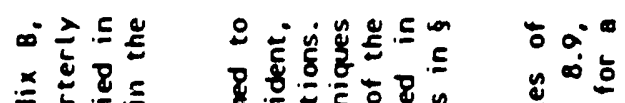

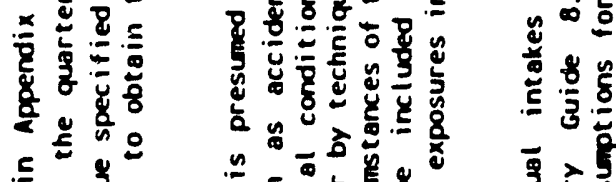

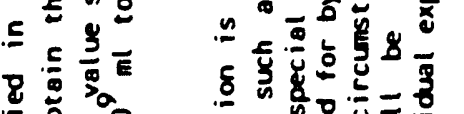

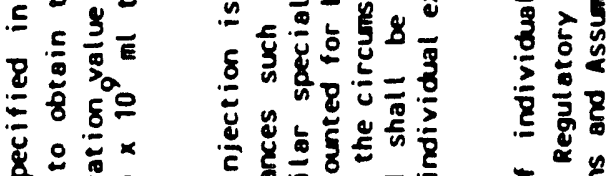

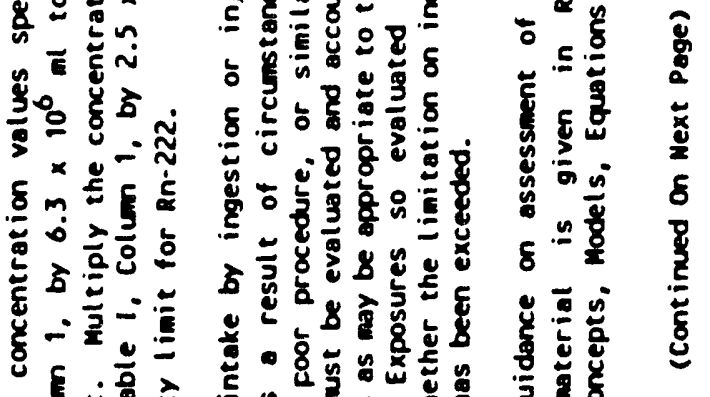

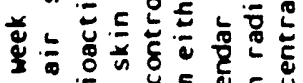

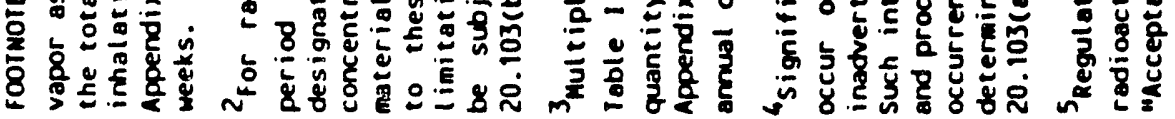




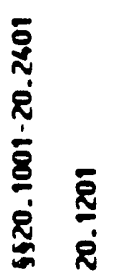

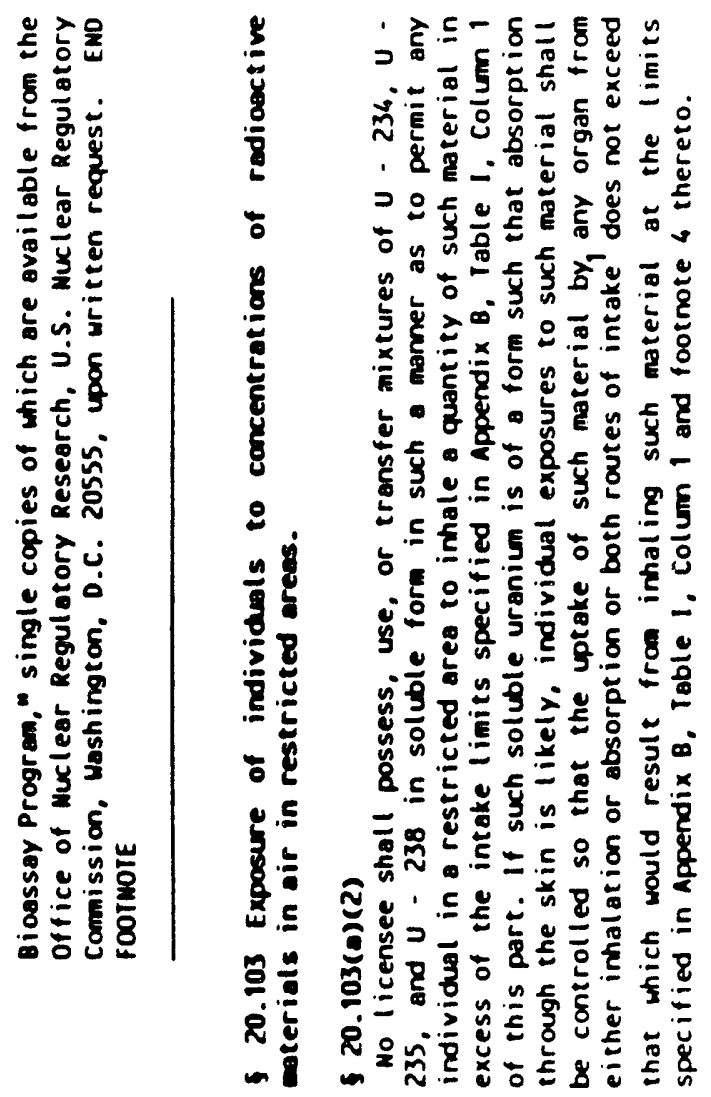

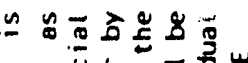

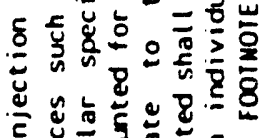

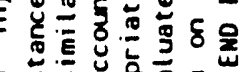

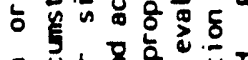

18

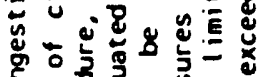

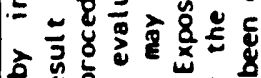

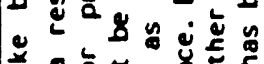

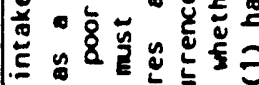

ट

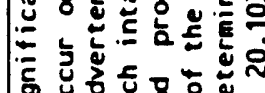

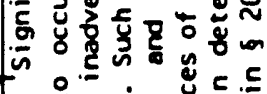

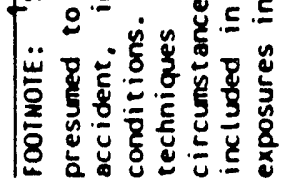

5
8
1
1
வ்
2

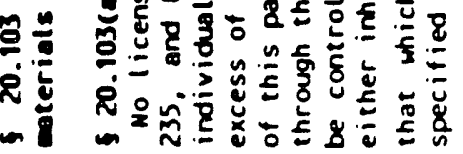




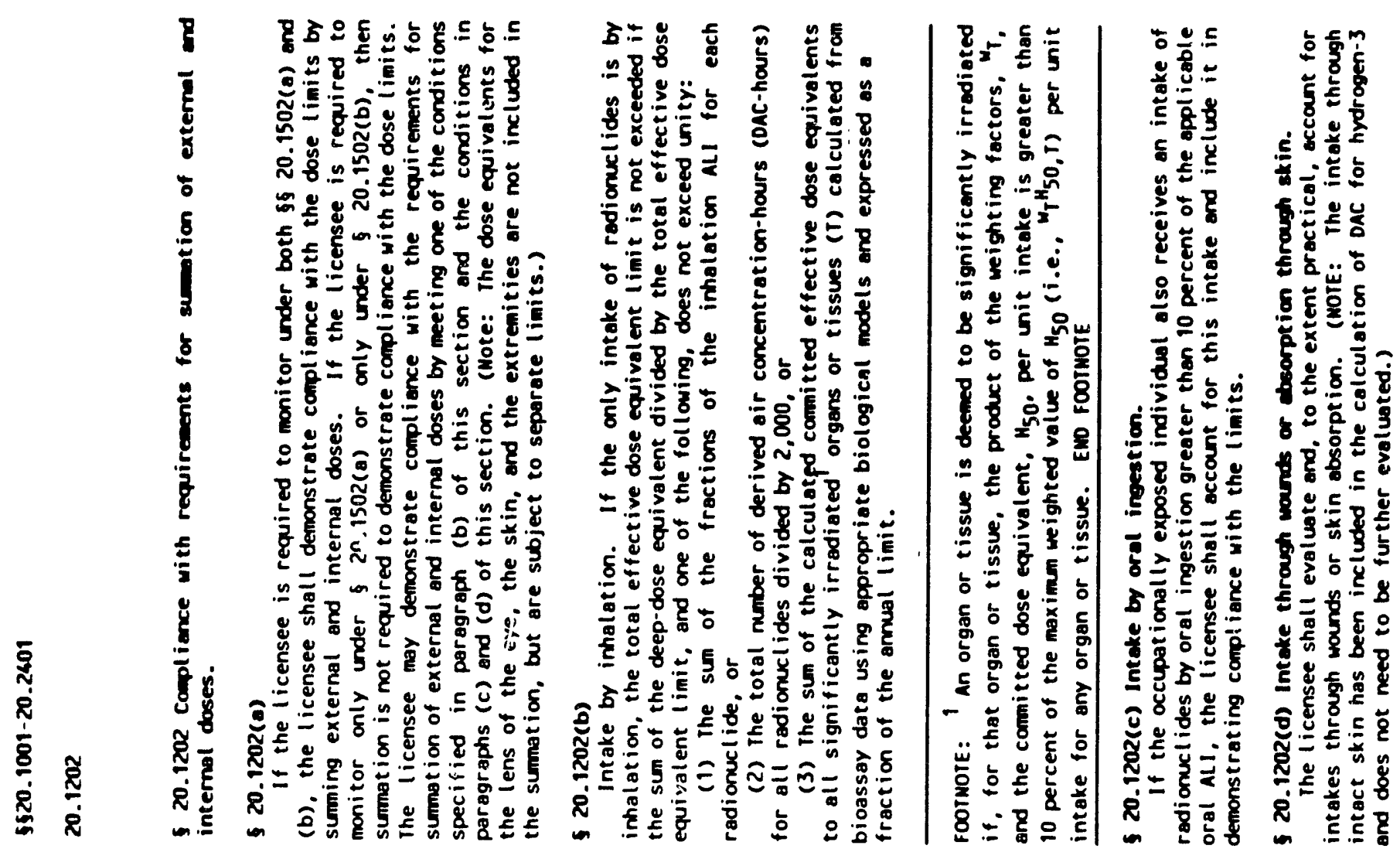



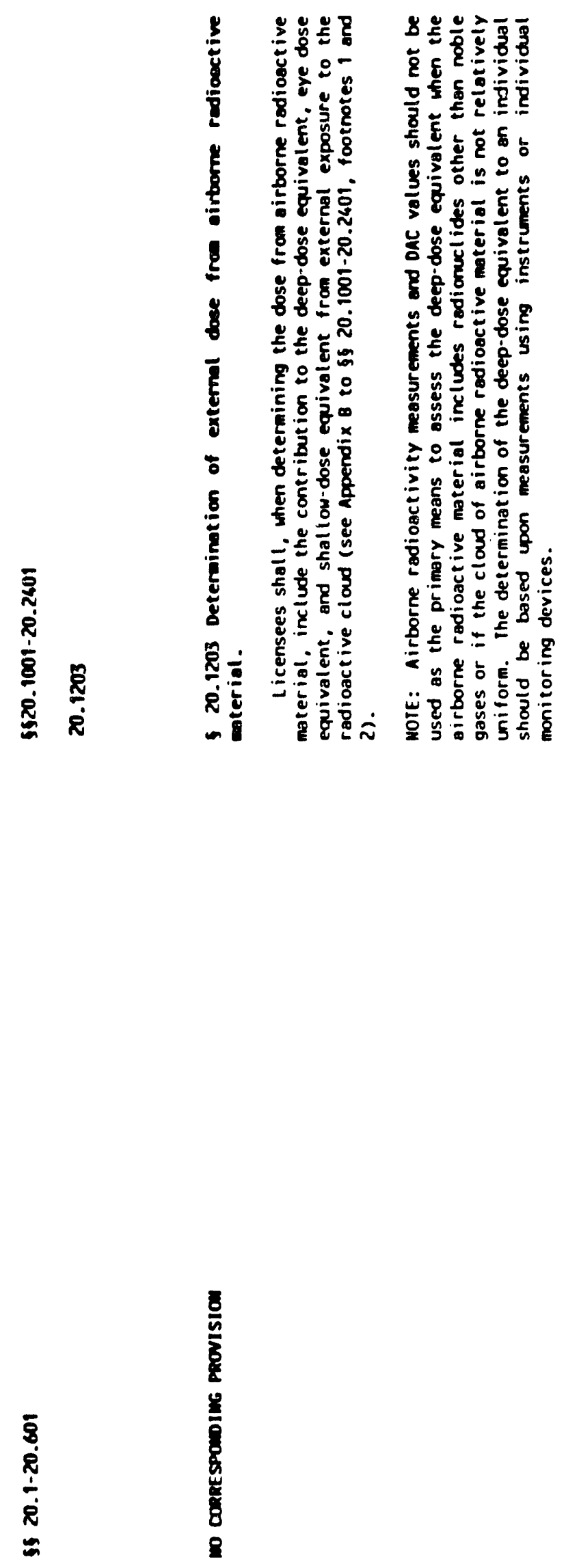


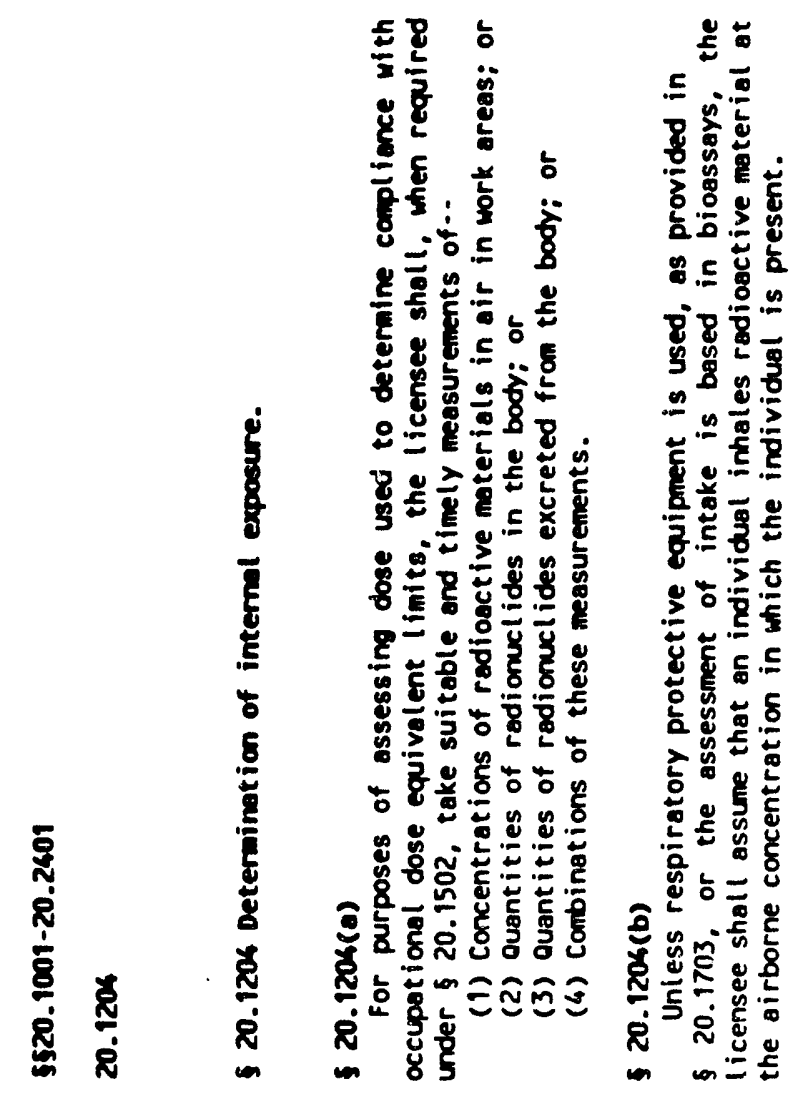

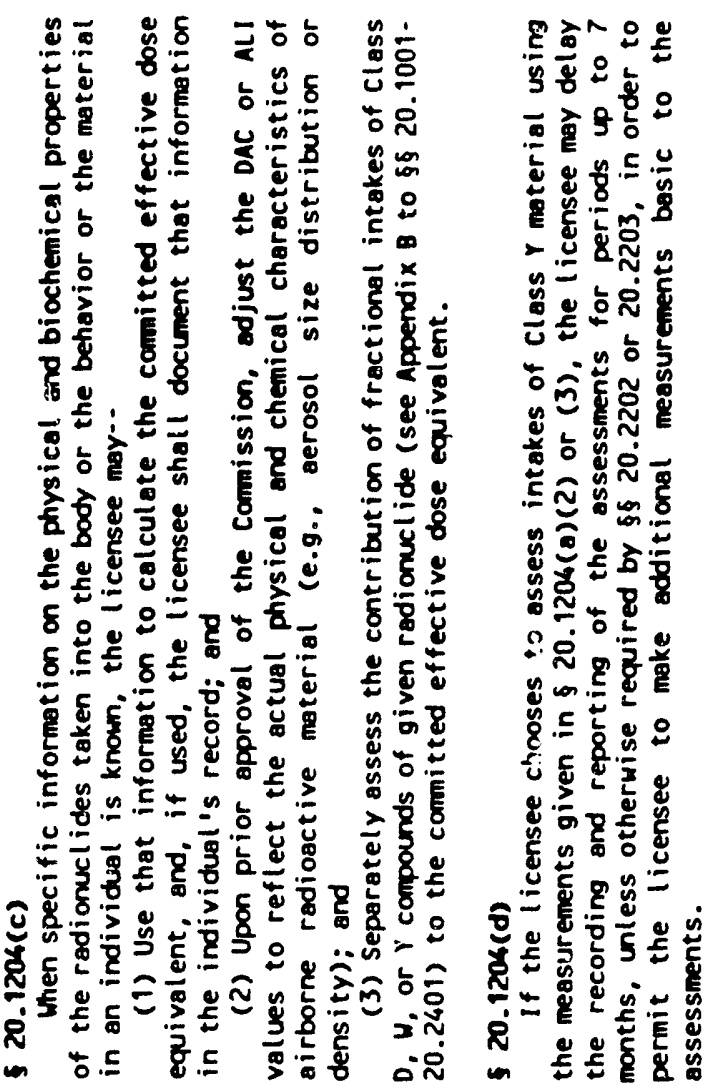

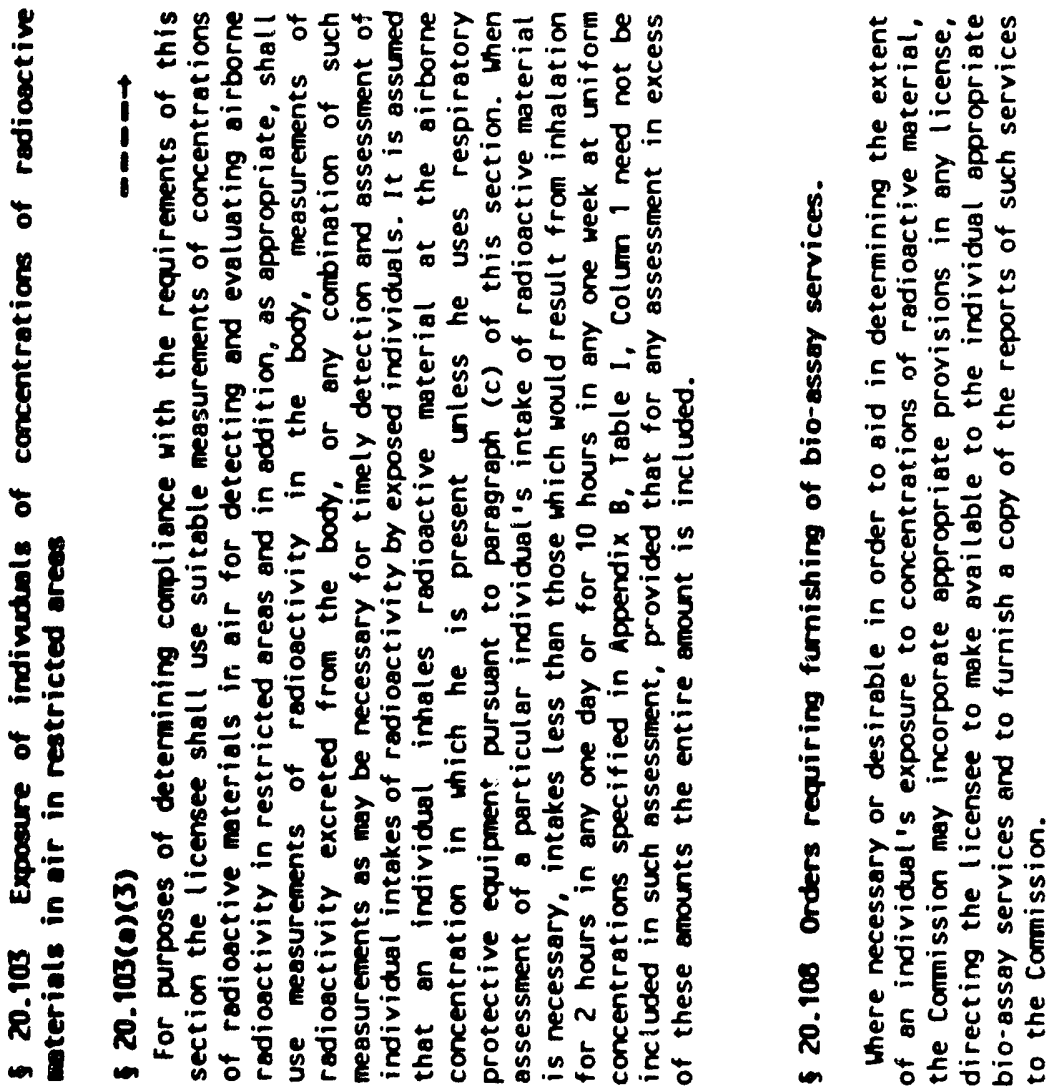




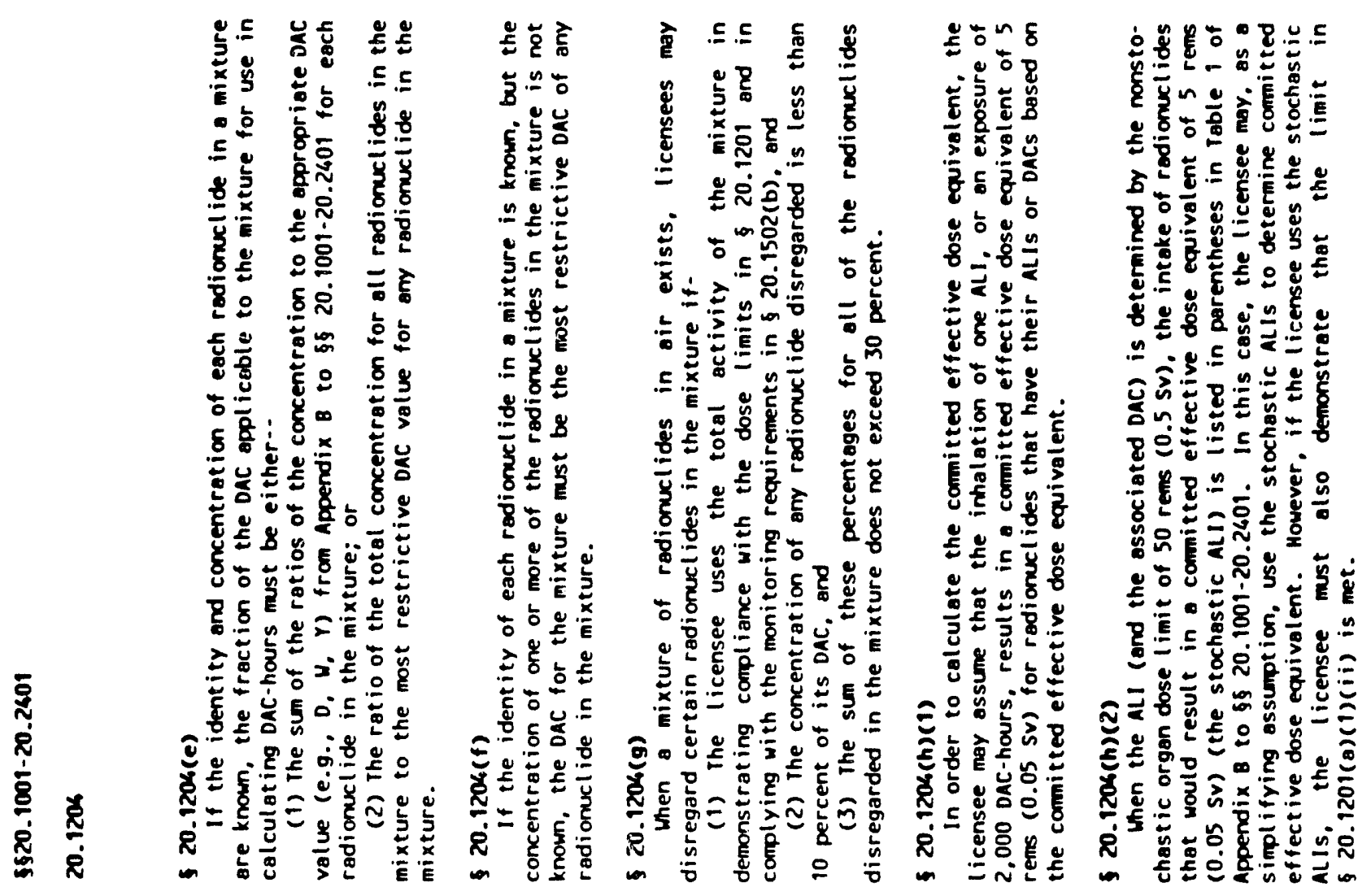




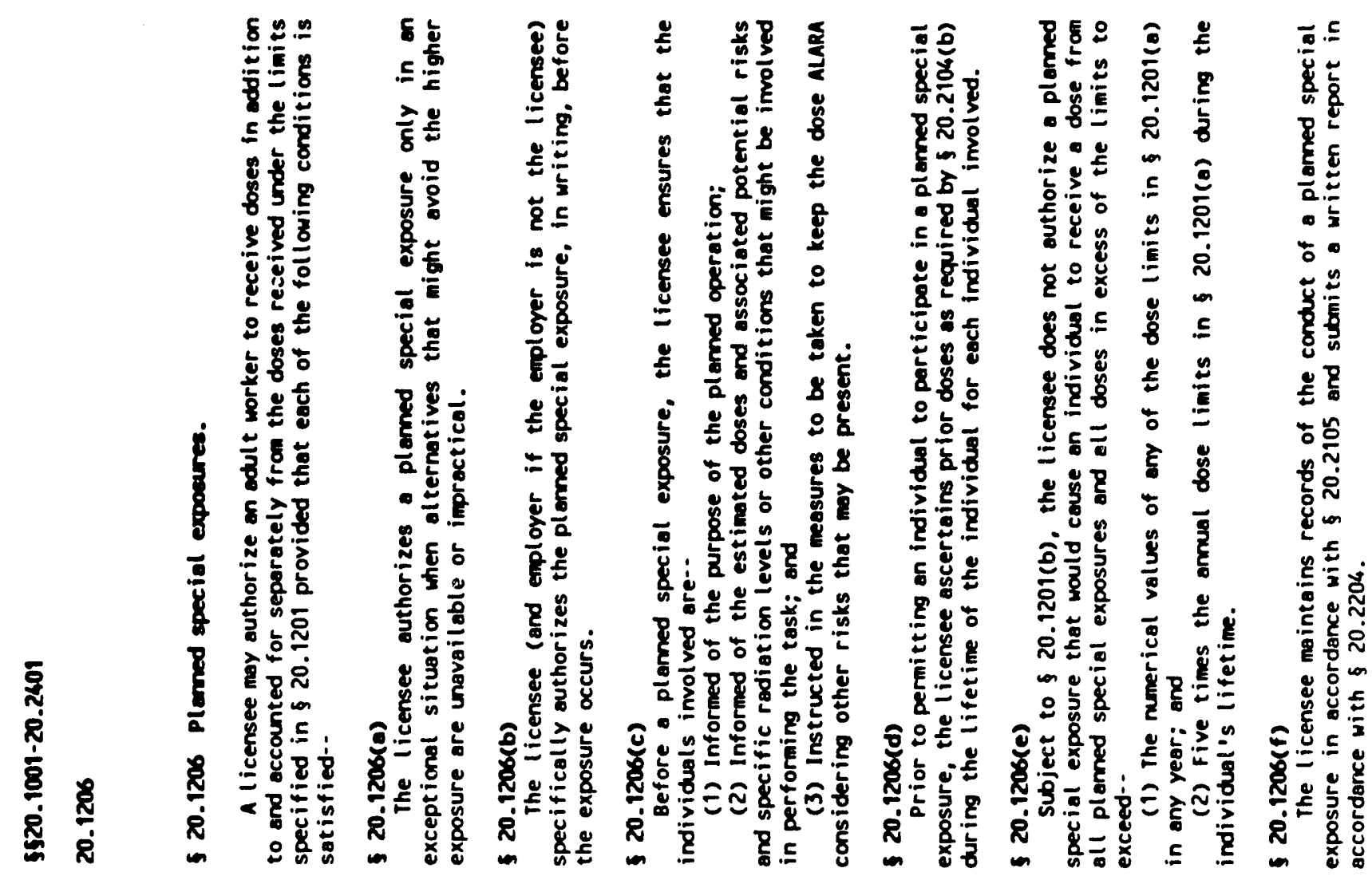




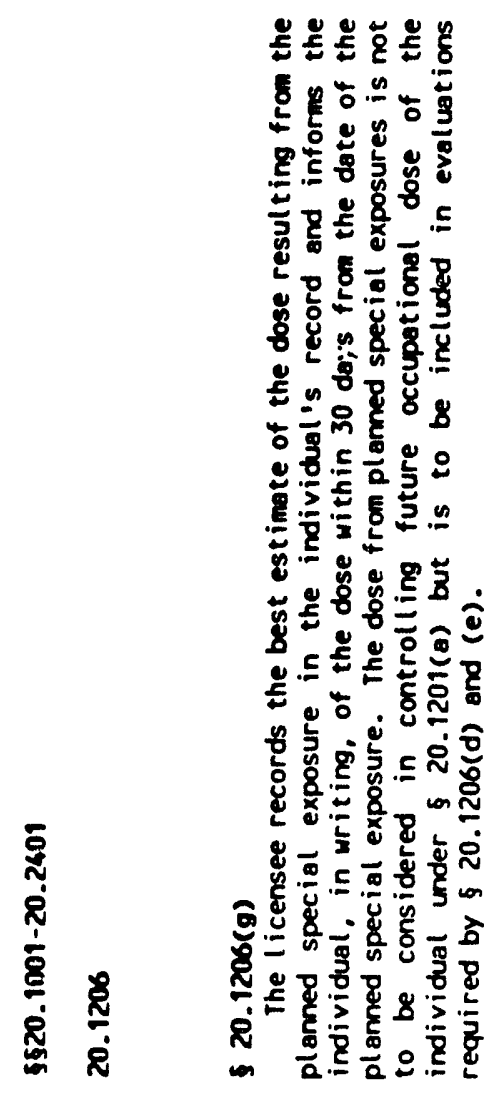

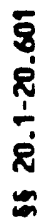




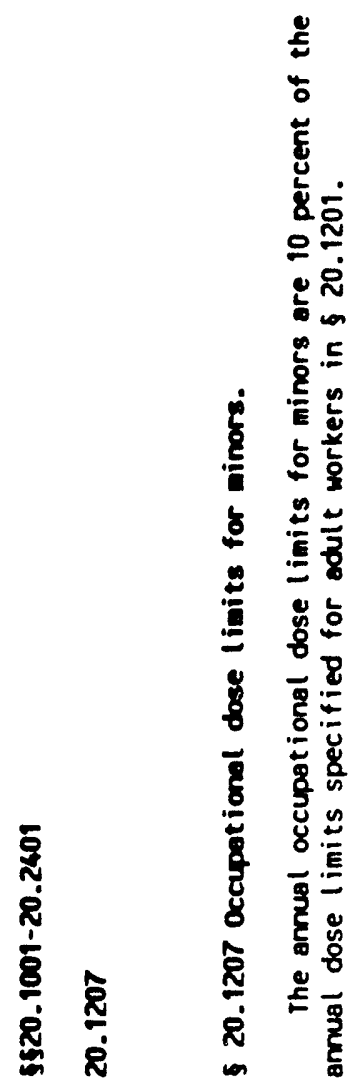

m

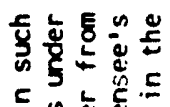

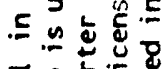

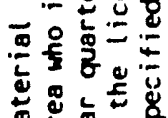

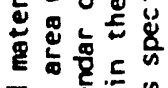

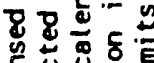

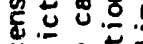

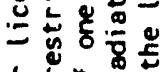

这家

我 8 \%

产造㝴

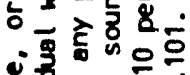

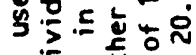

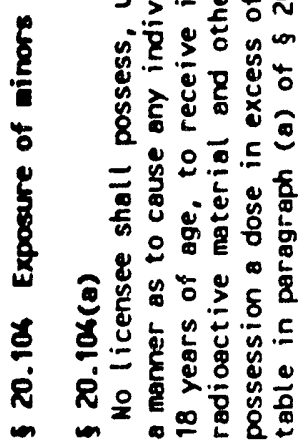

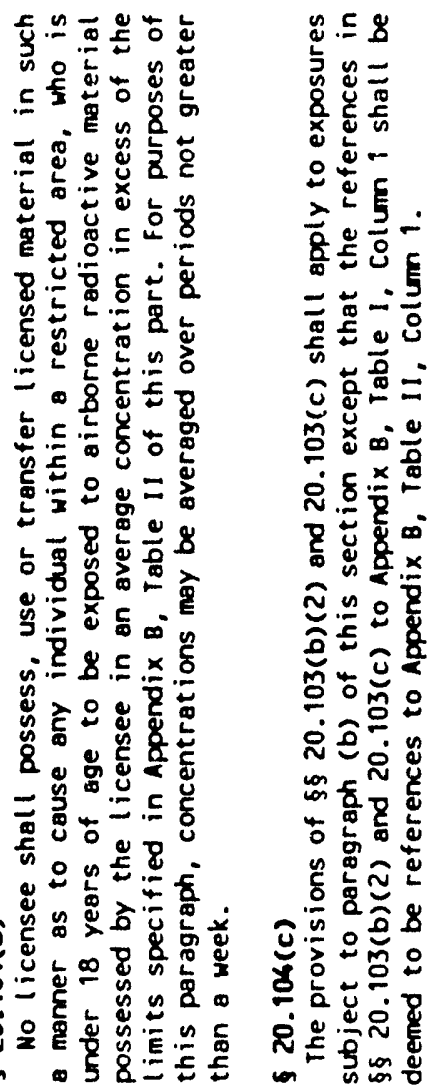




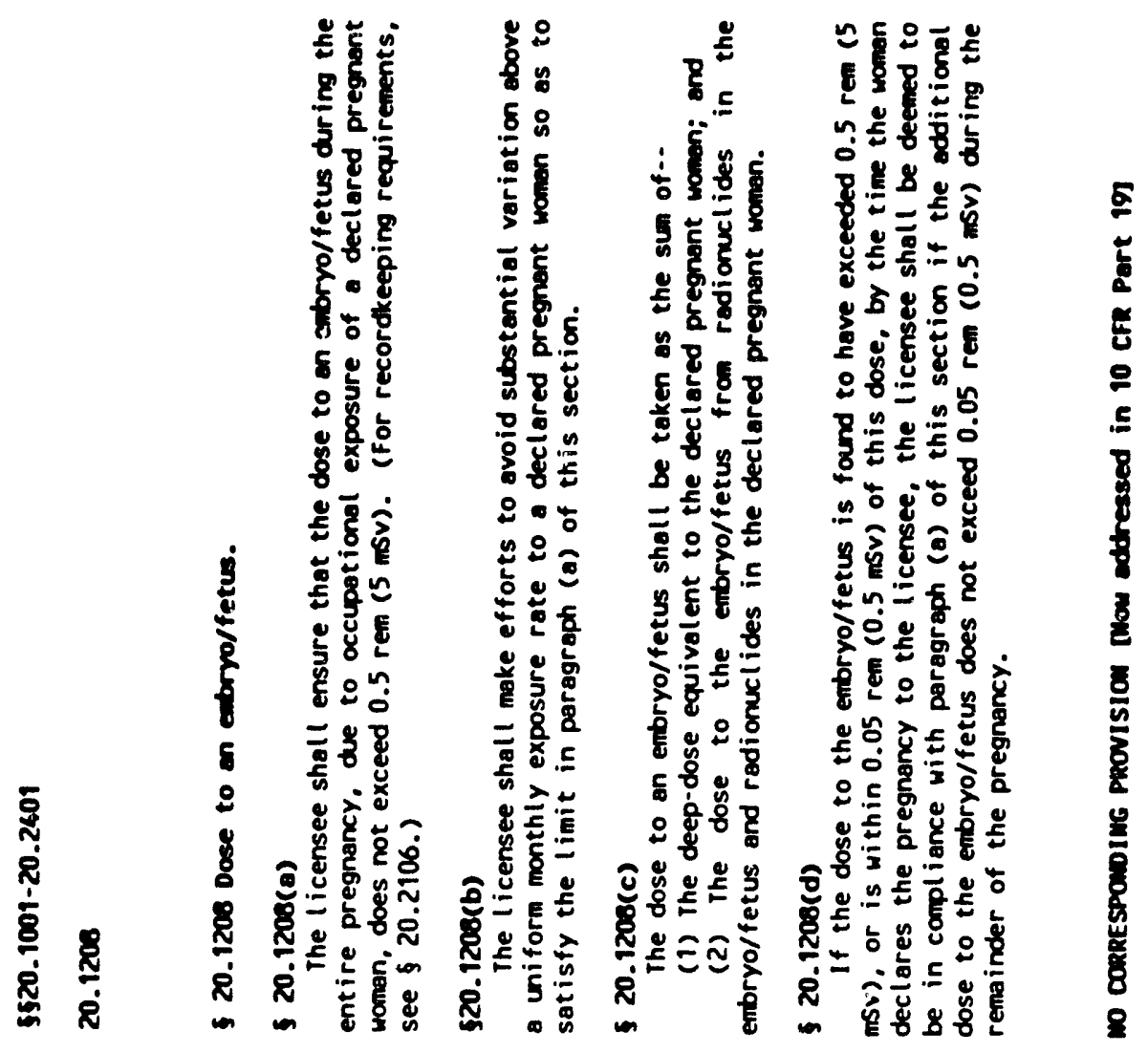

m

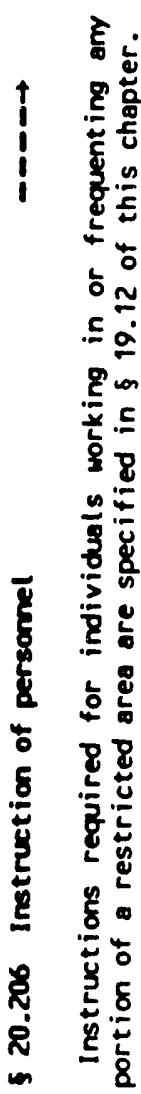



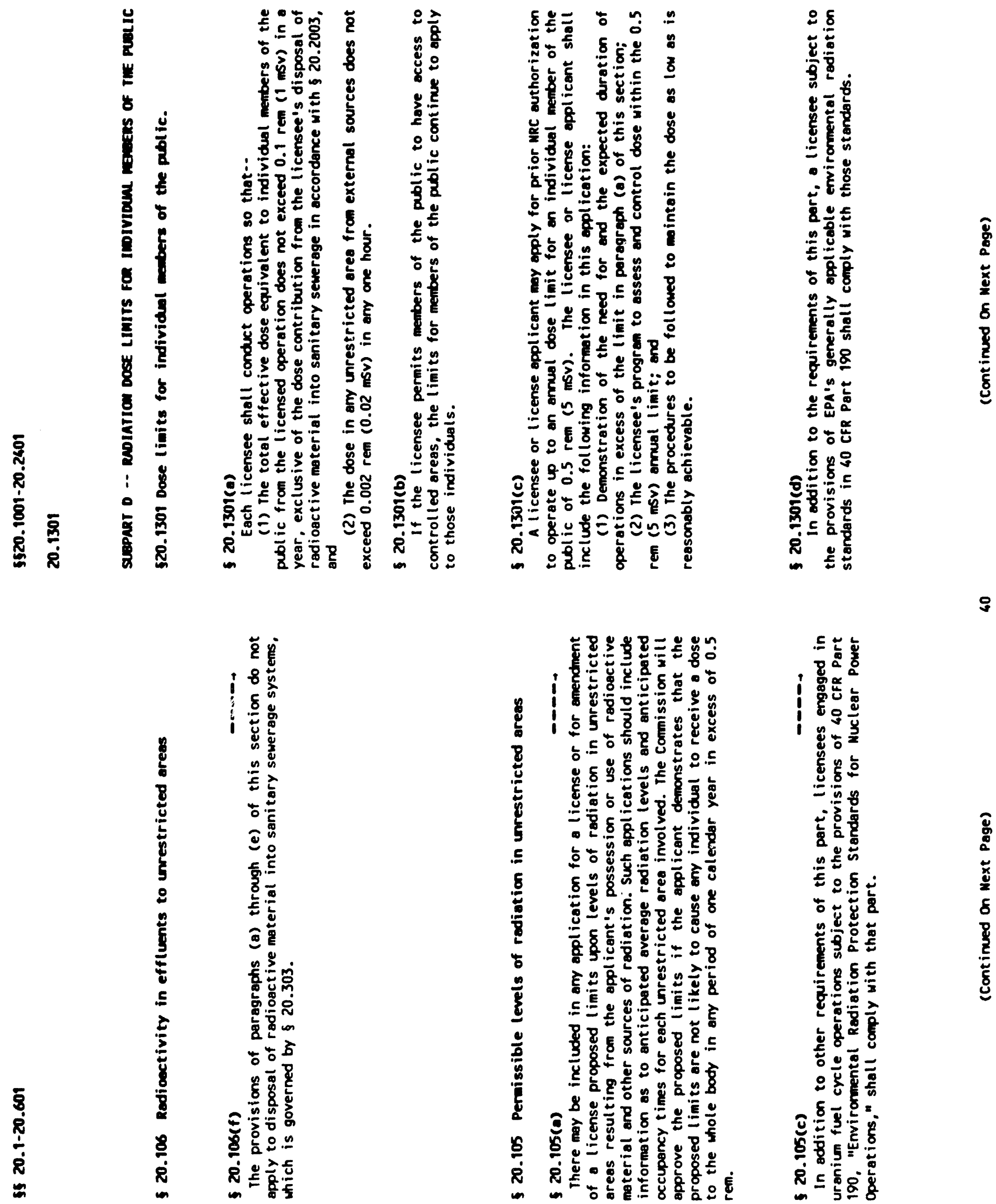

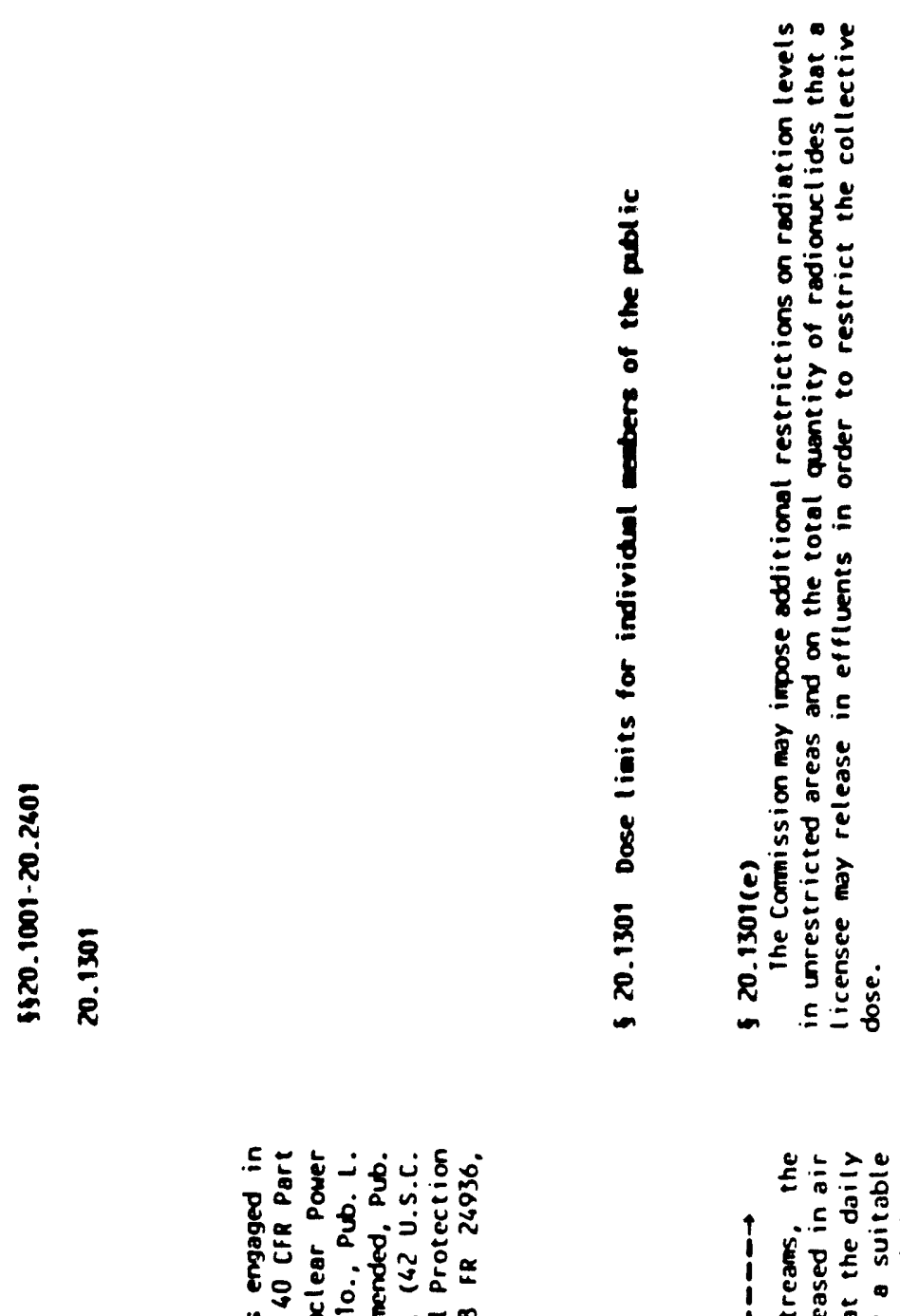

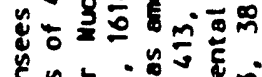

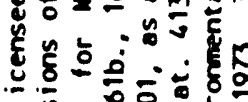

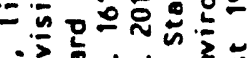

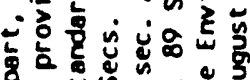

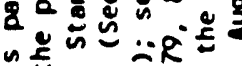

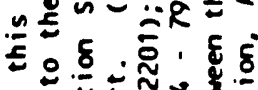

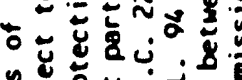

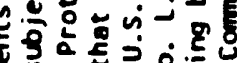

क 8 ₹

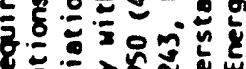

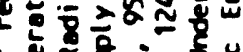

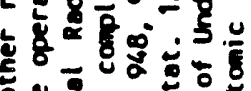

$\overline{8}$

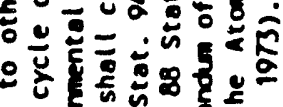
ง

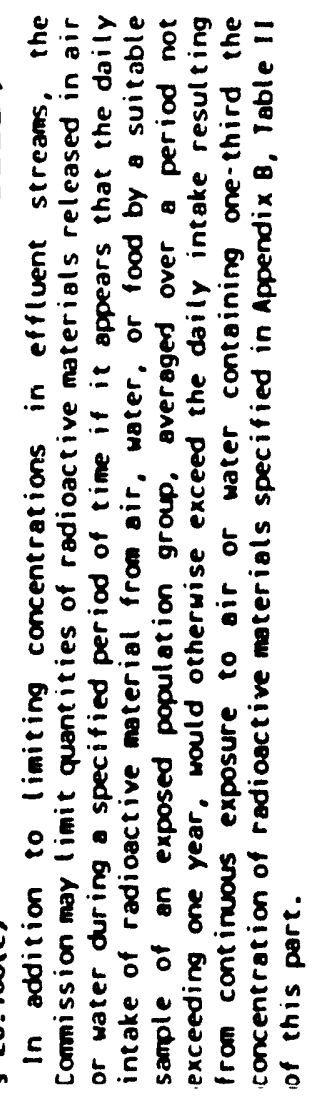



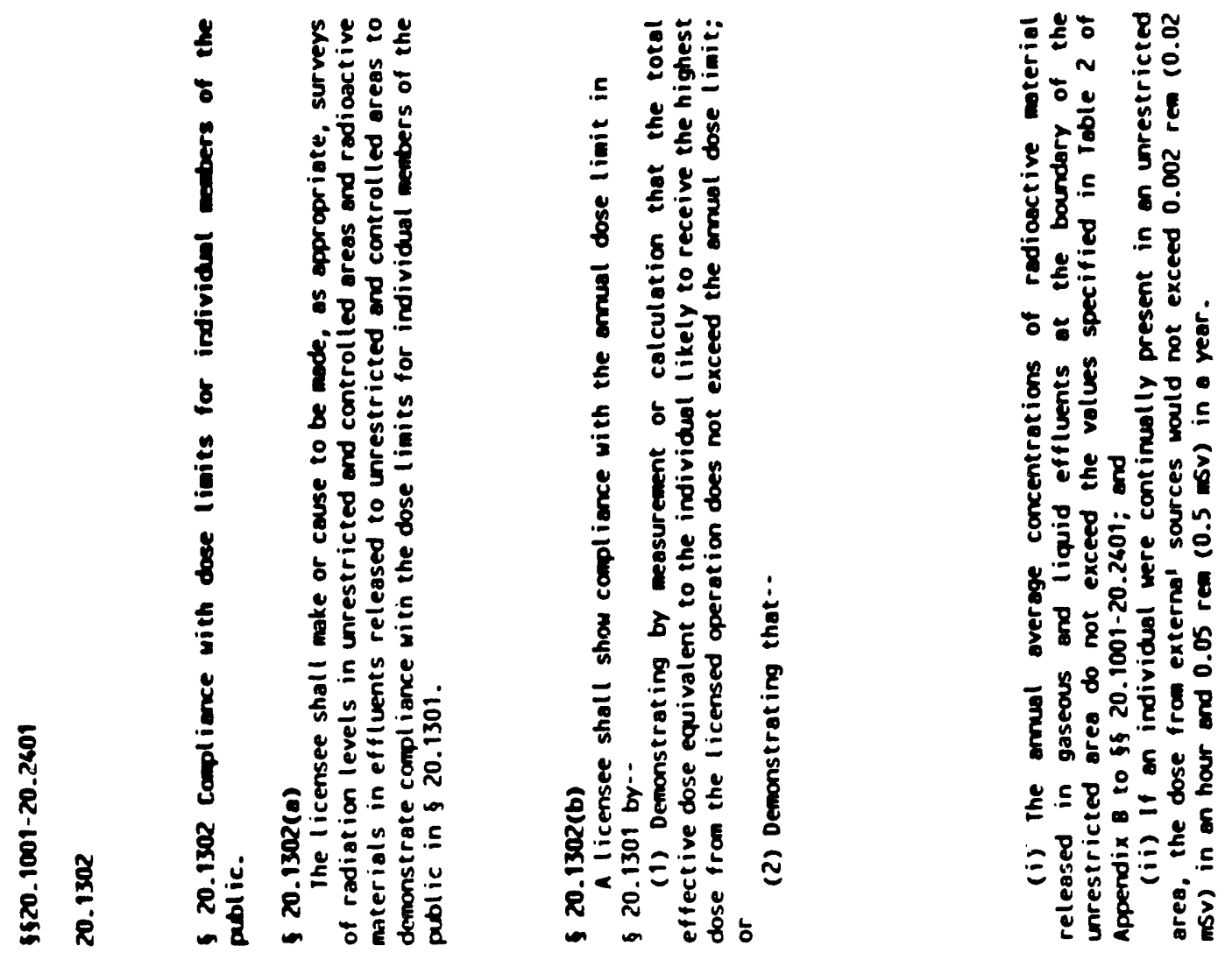

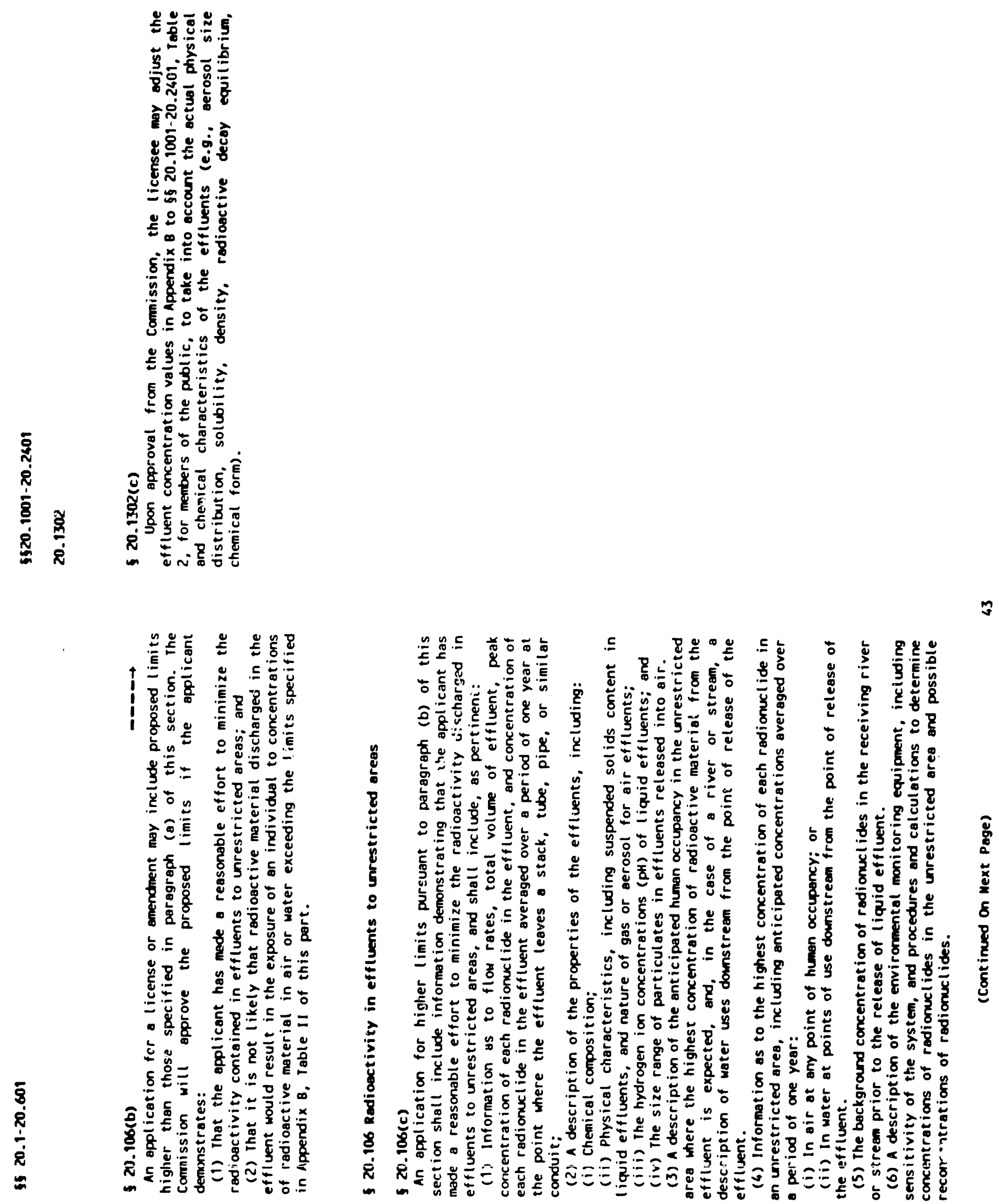

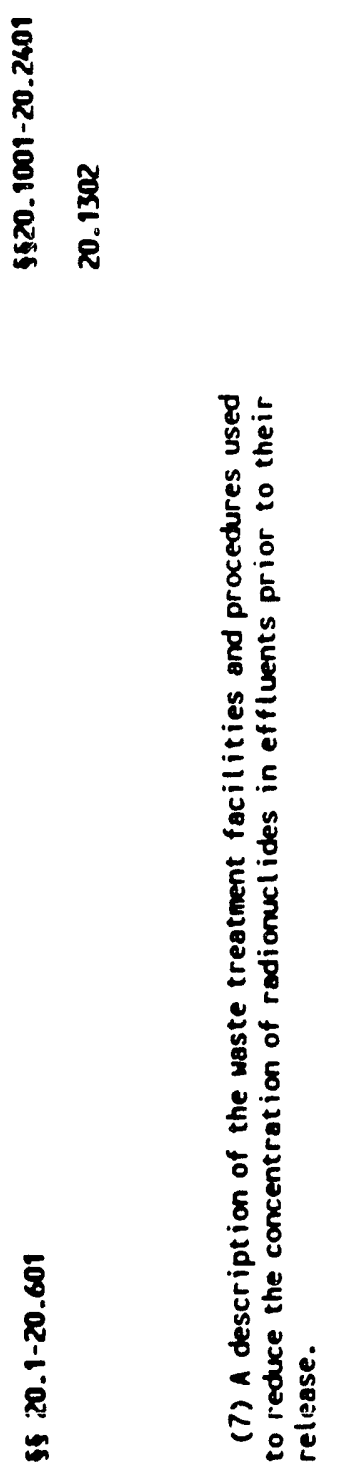


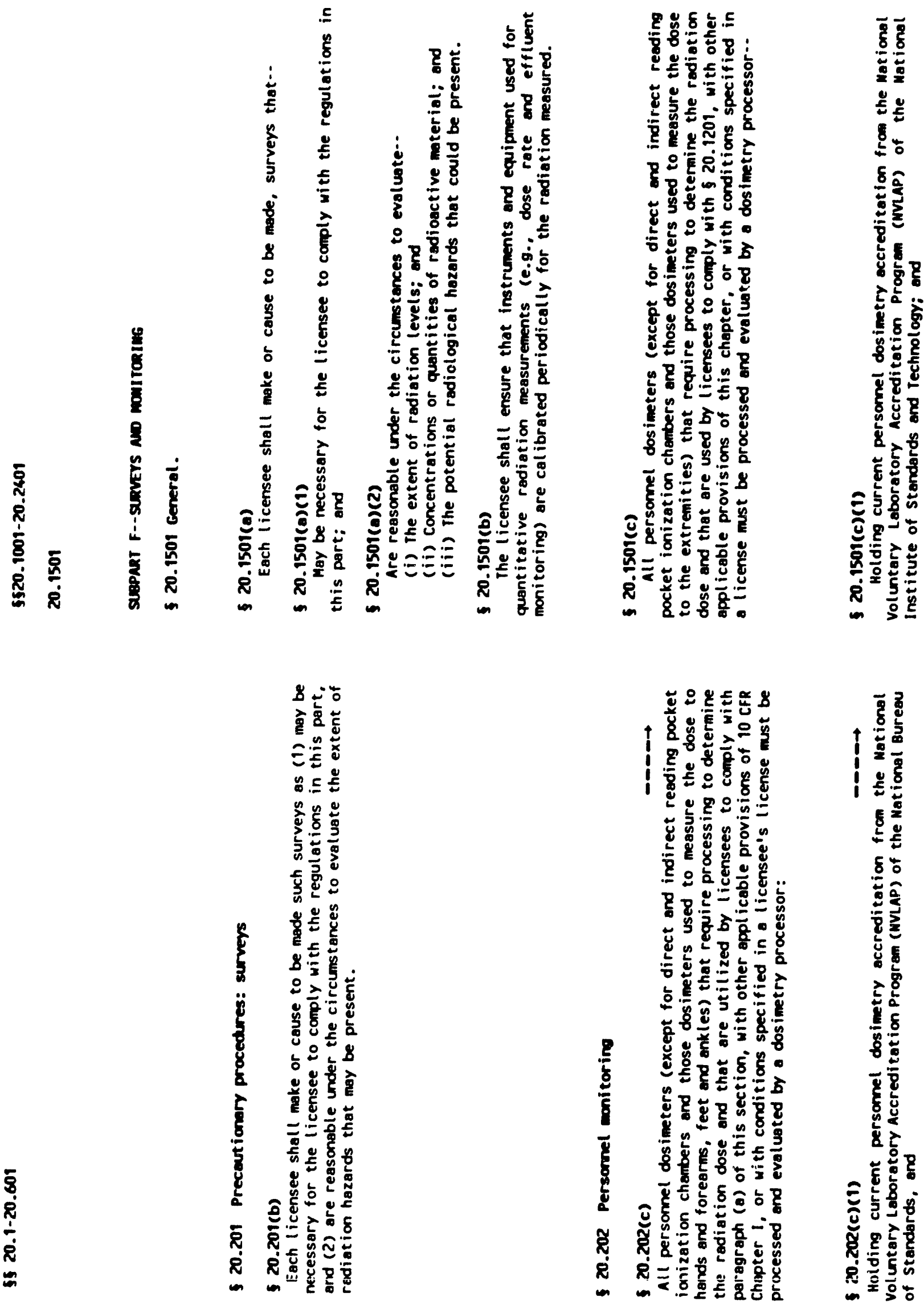



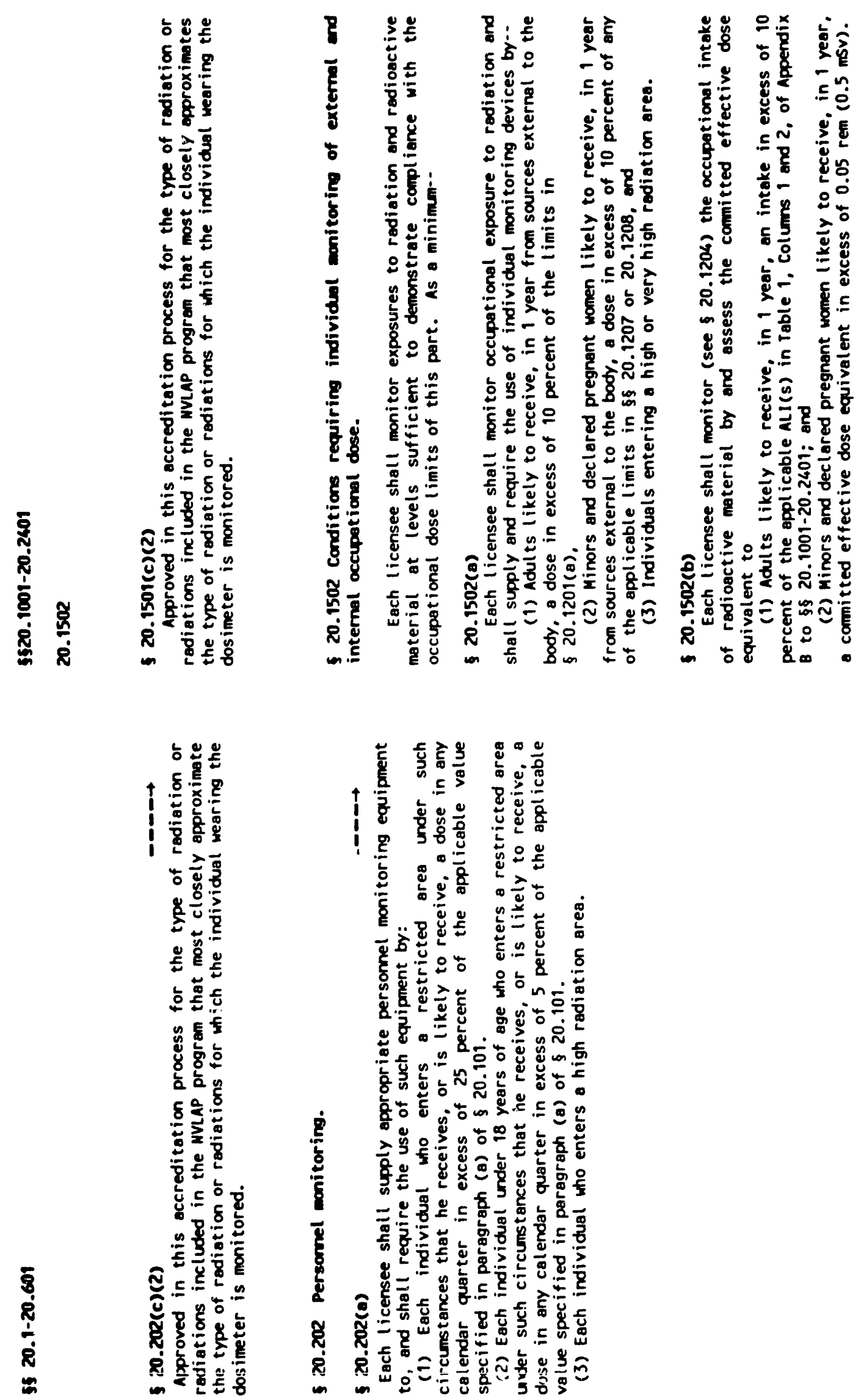


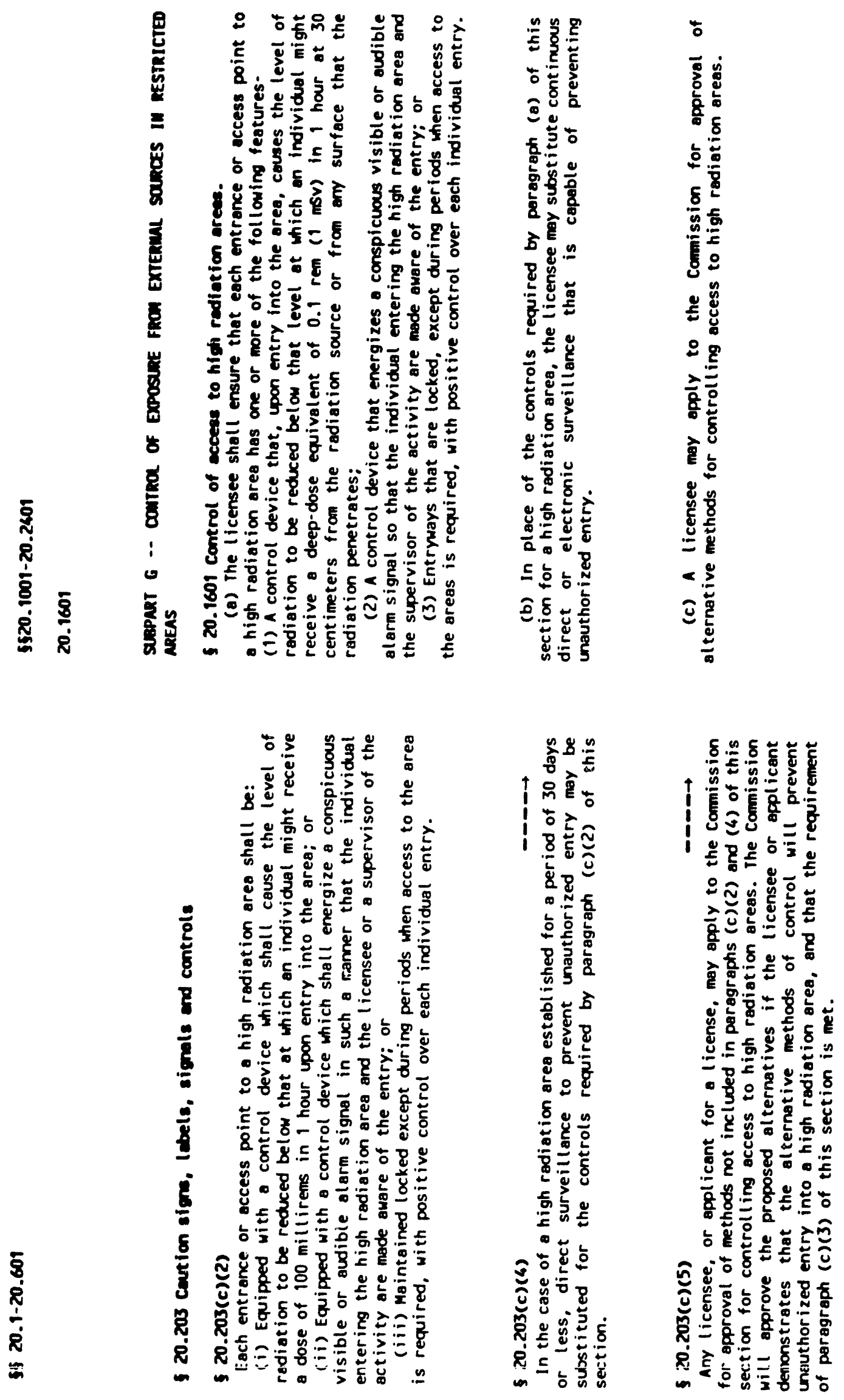



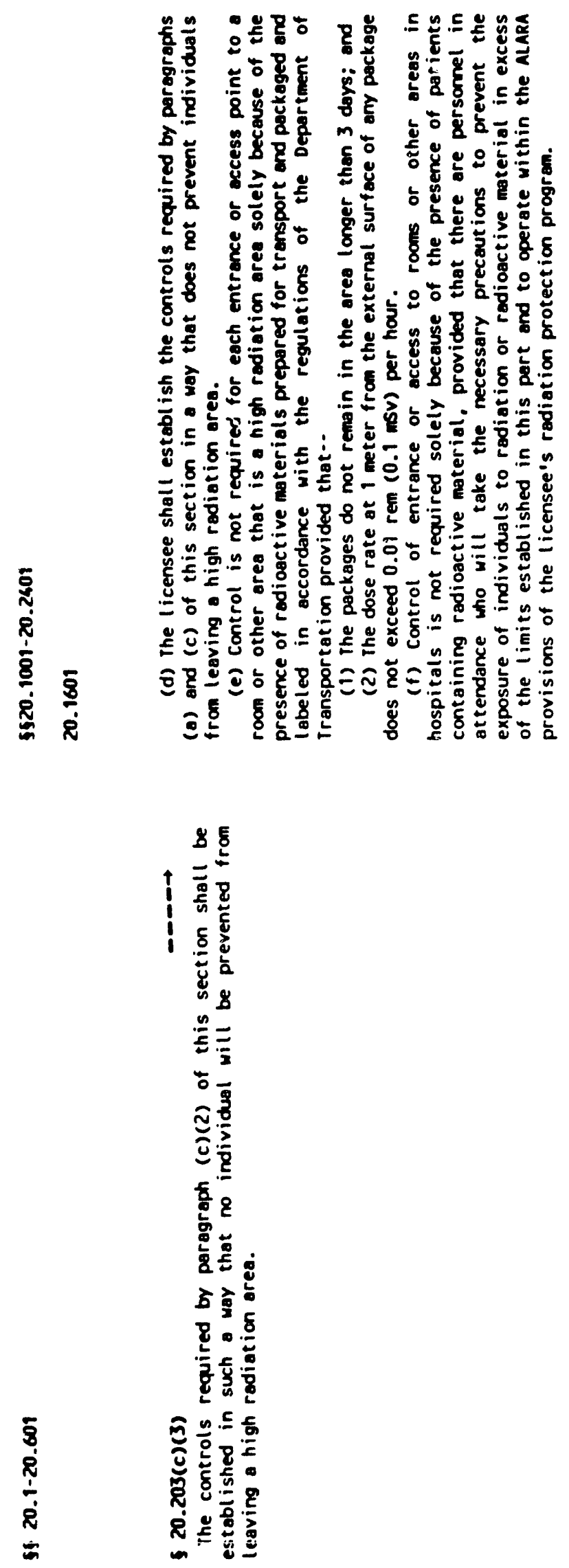


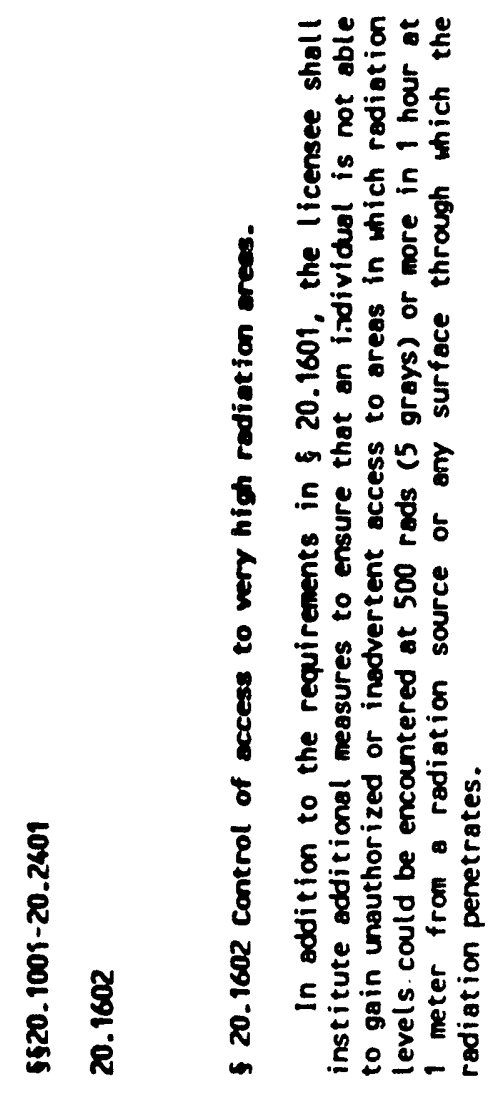



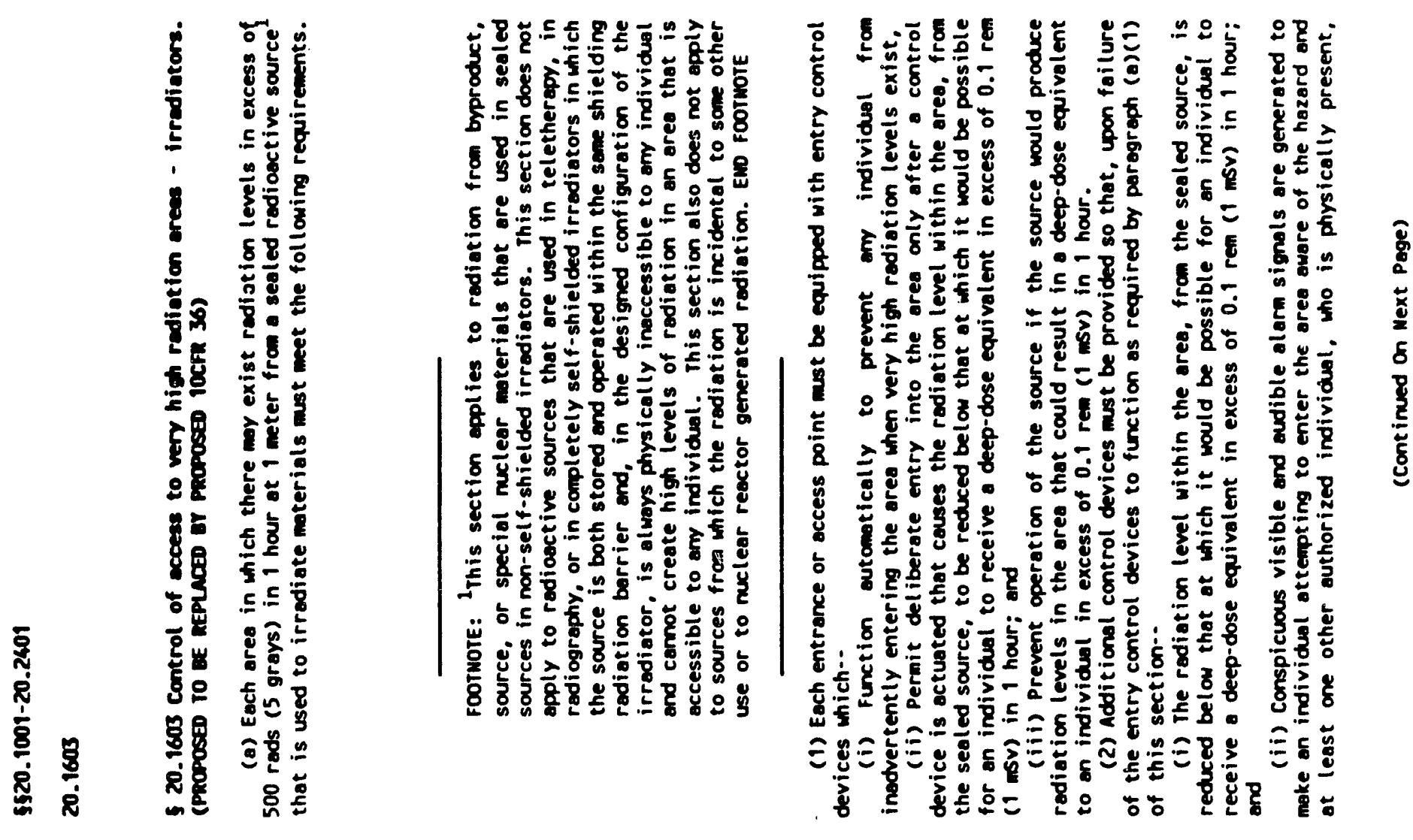

요

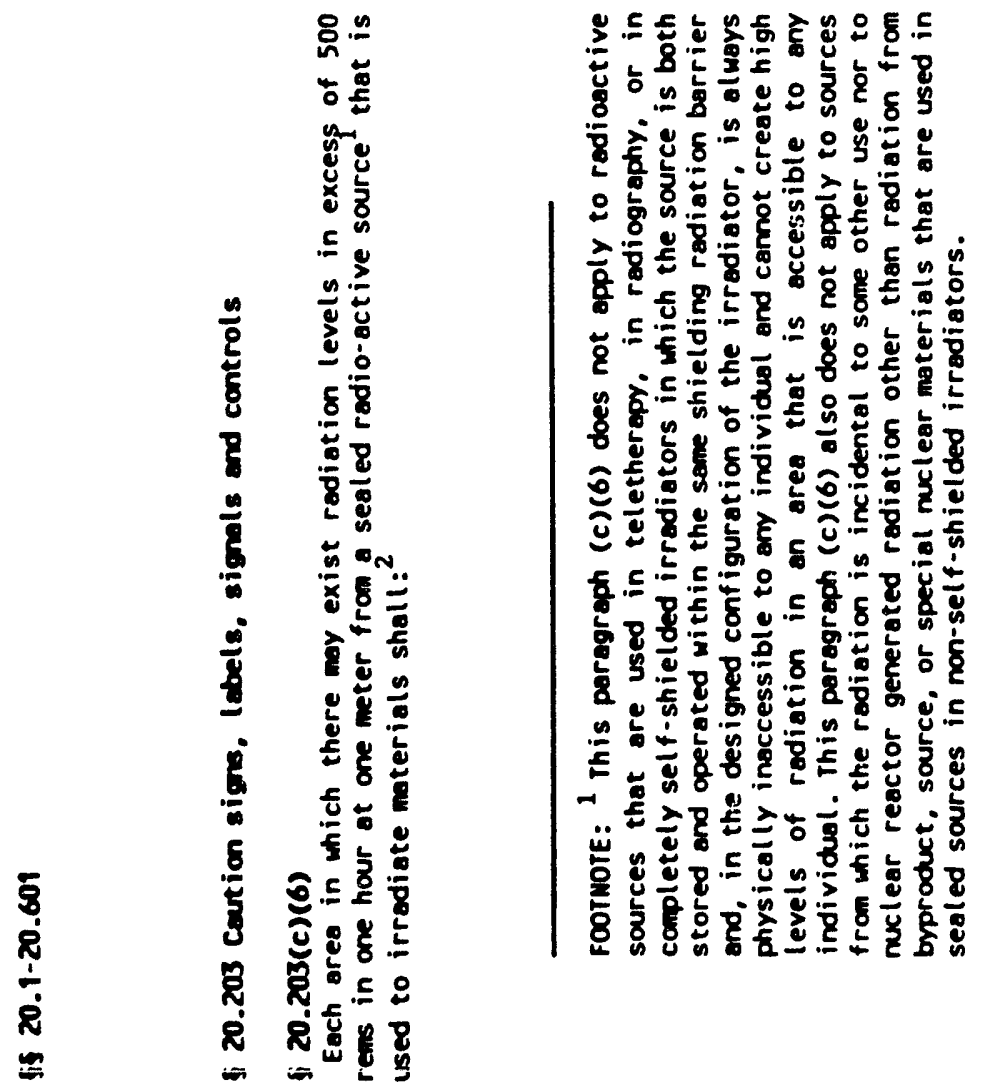
0

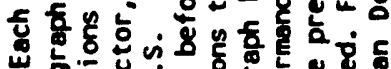

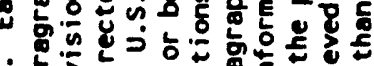

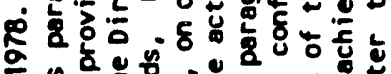

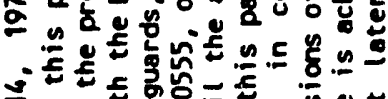

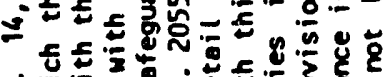

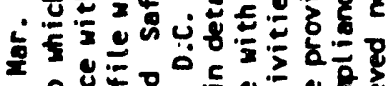

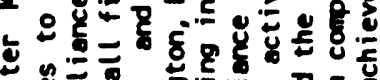
过

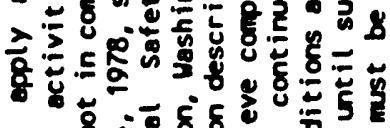

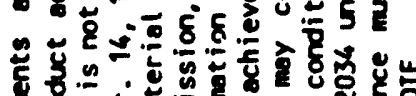

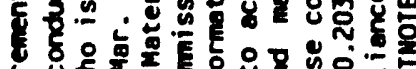

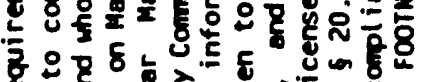

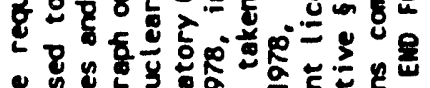

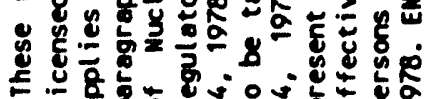

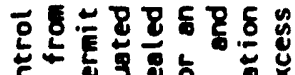

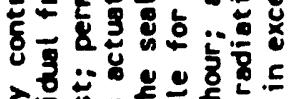
>든. है.

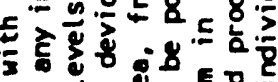
\&

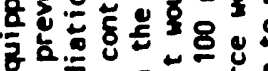

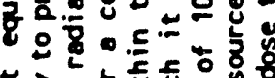

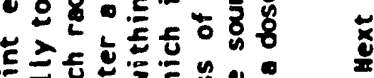

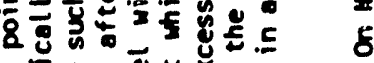
玤

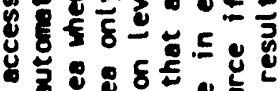

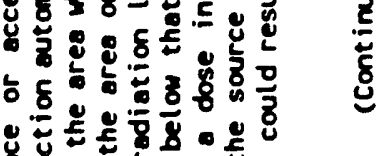
每

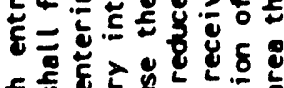
古竞 8 娄它

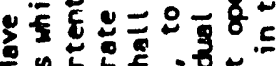

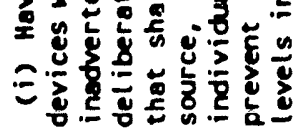




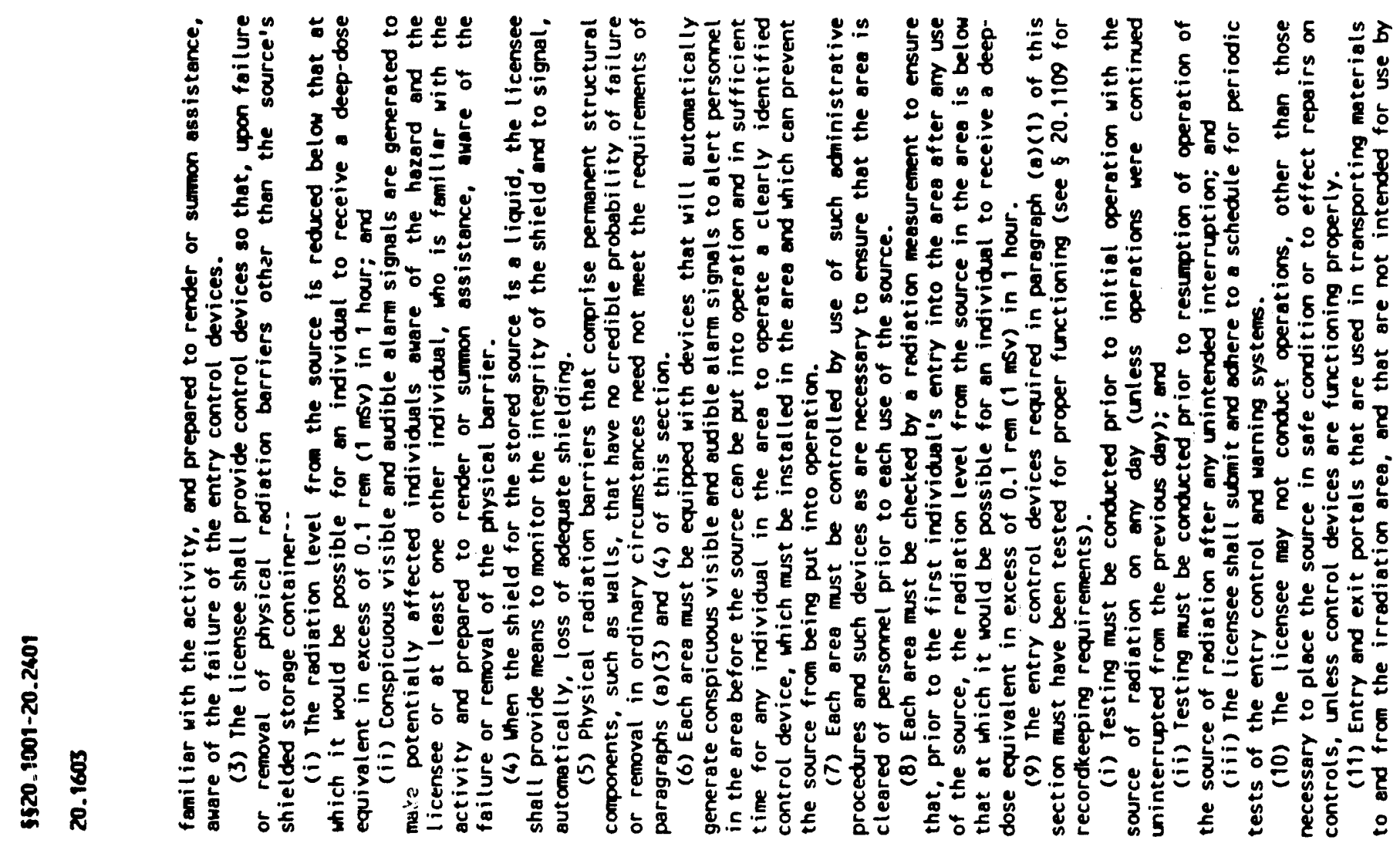

$\pi$

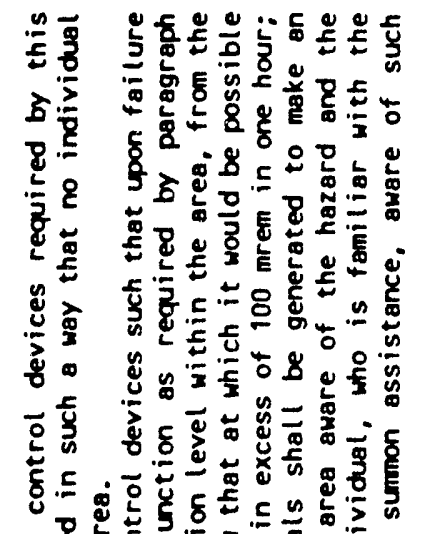

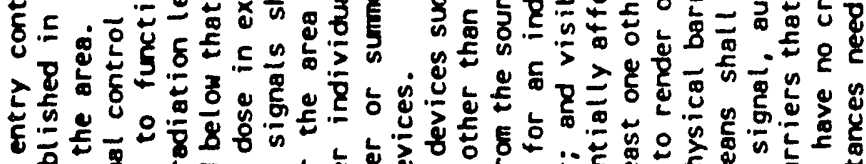

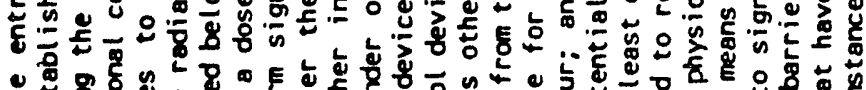
के

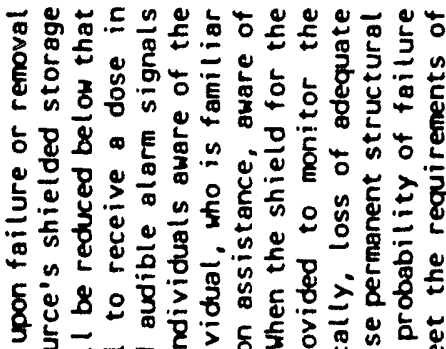

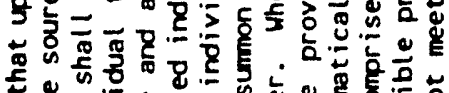

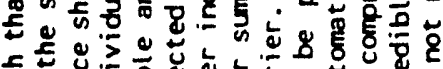

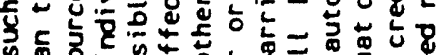

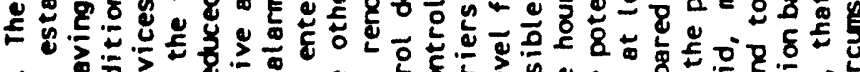

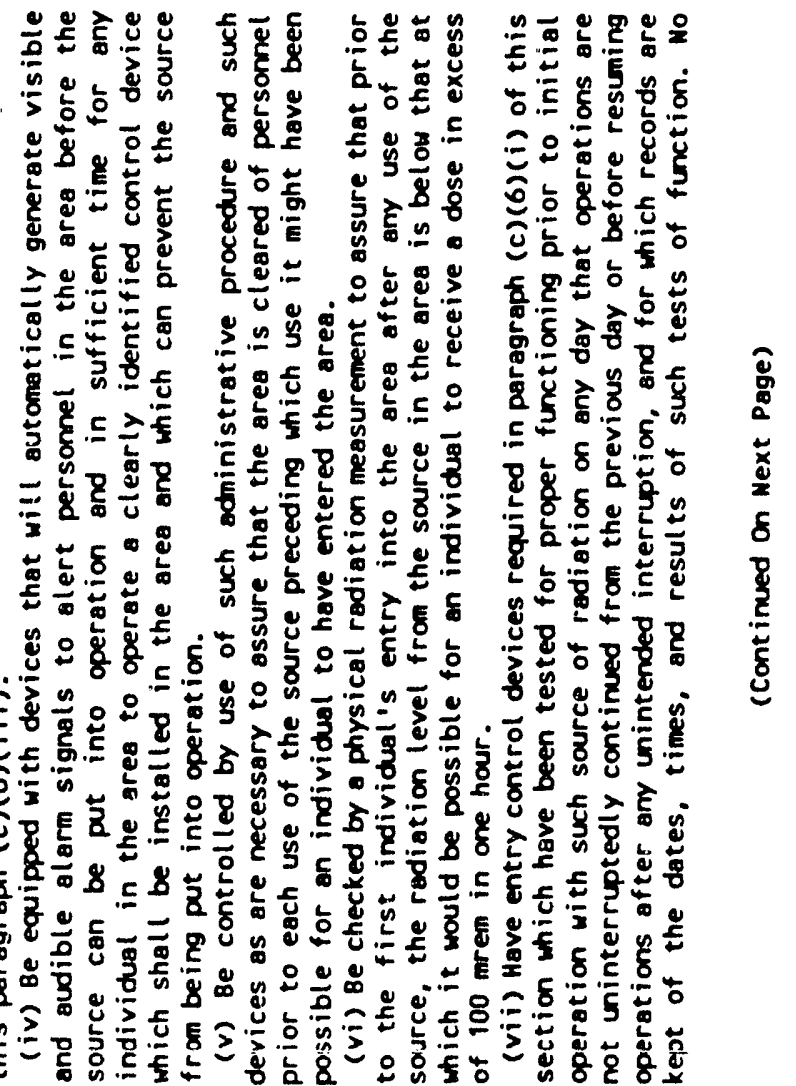



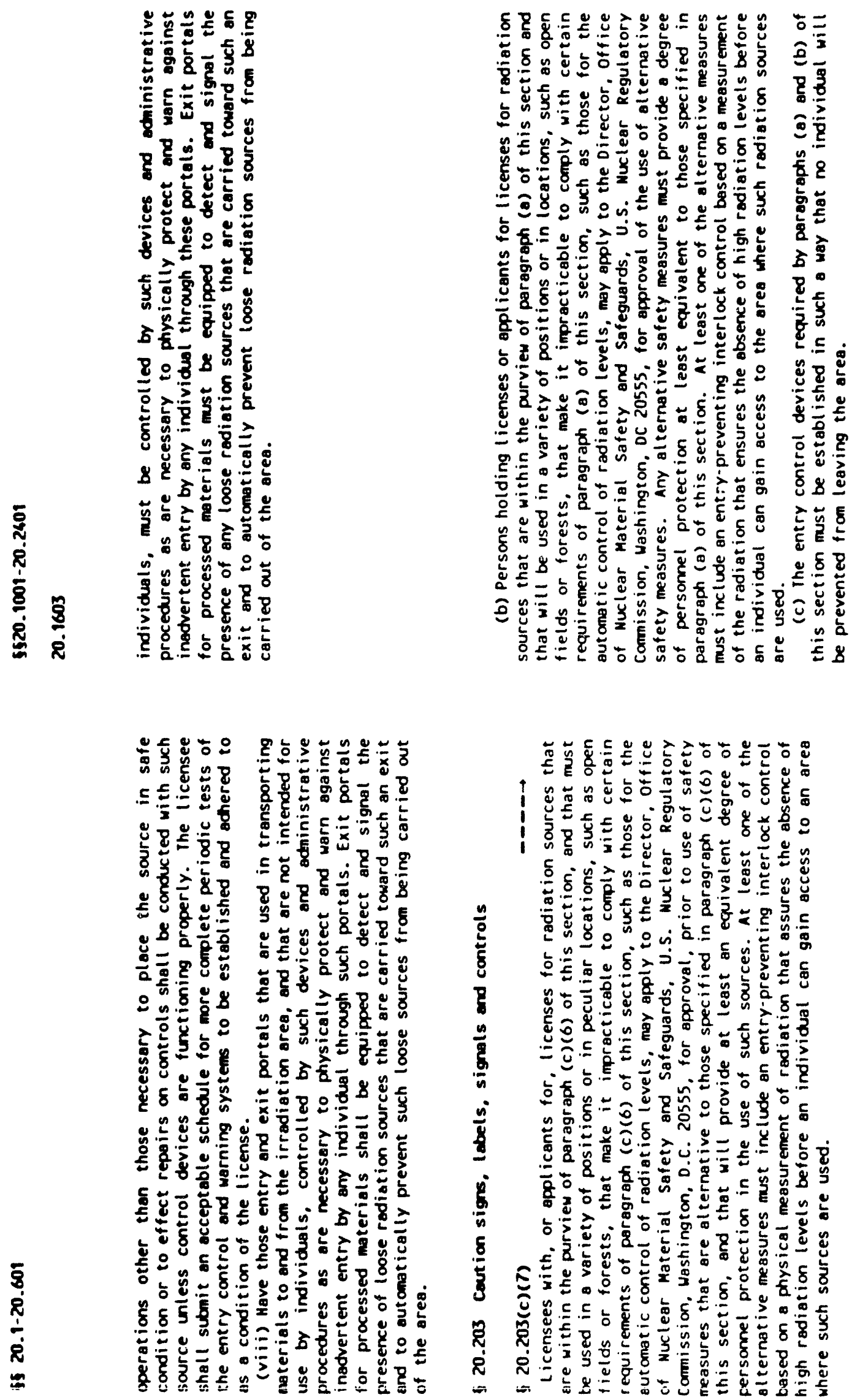

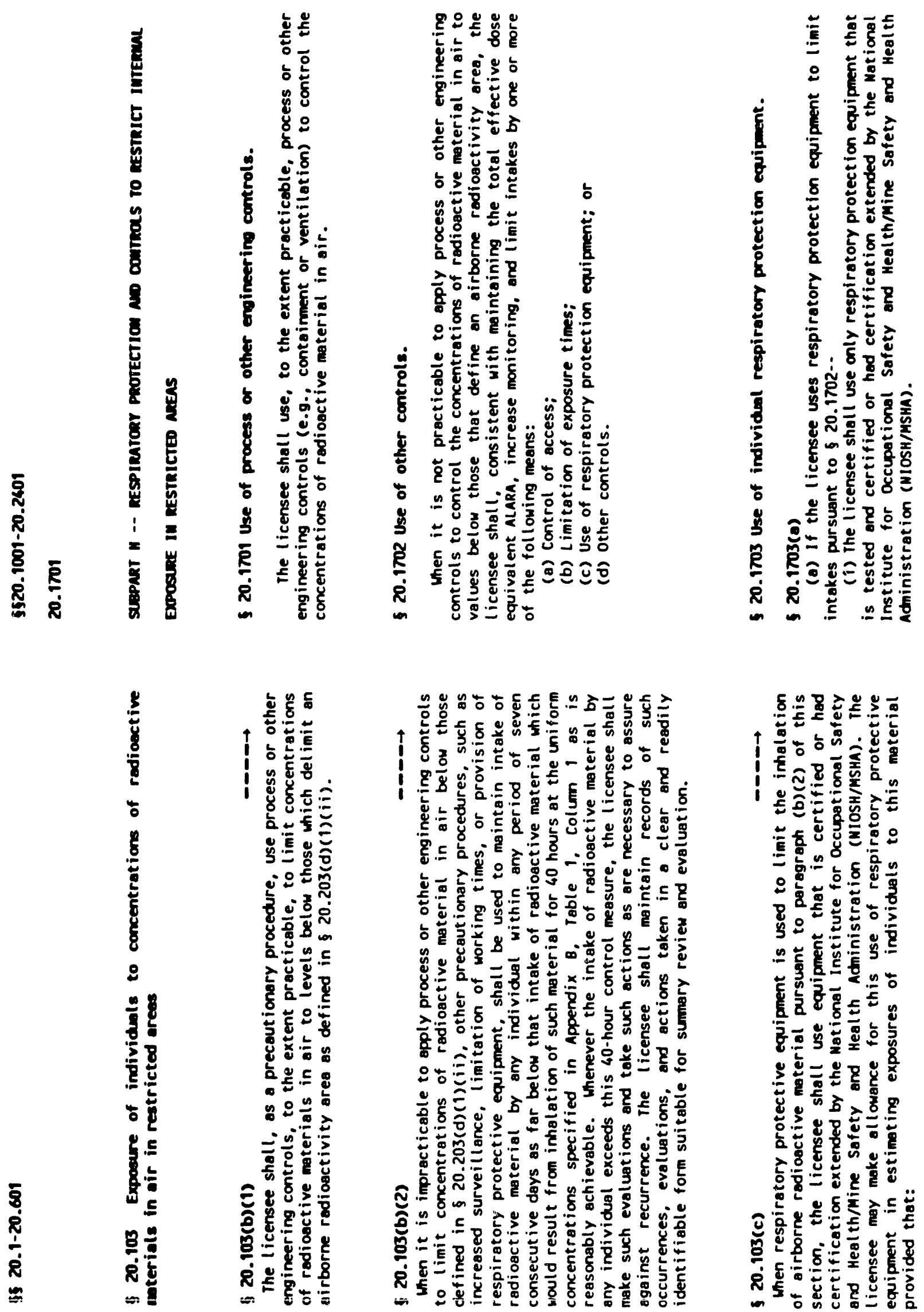

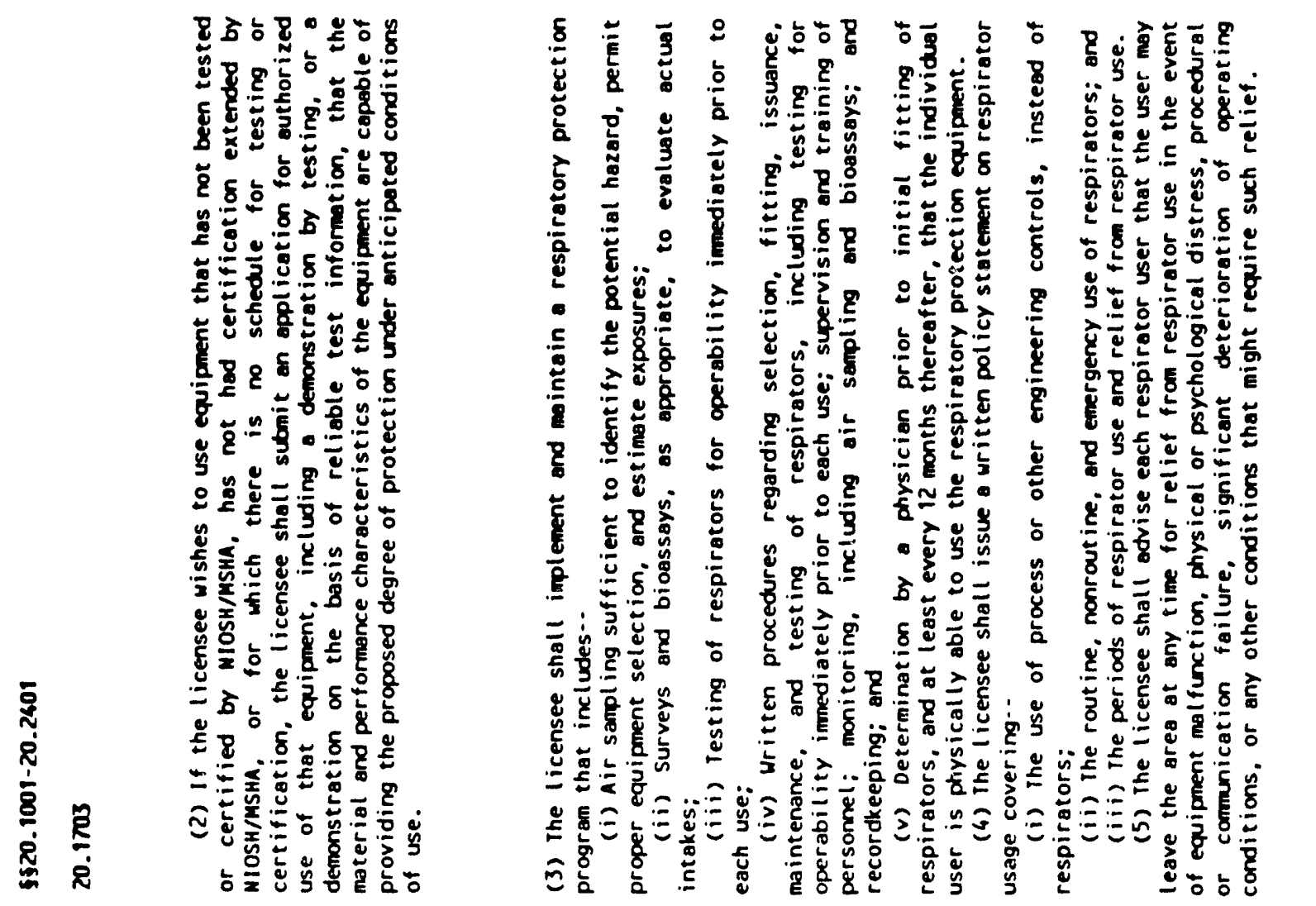

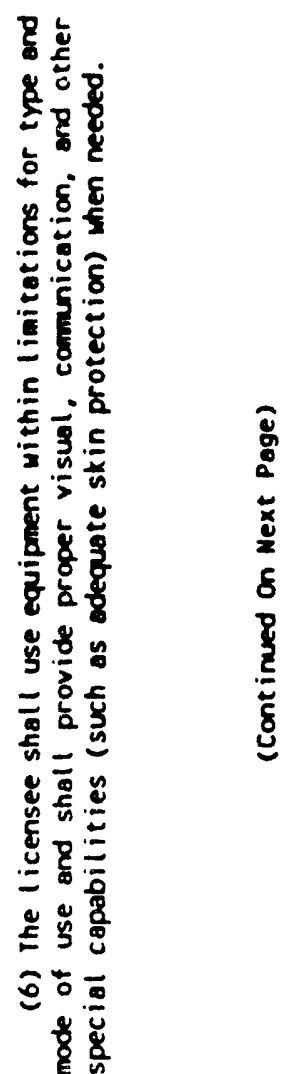
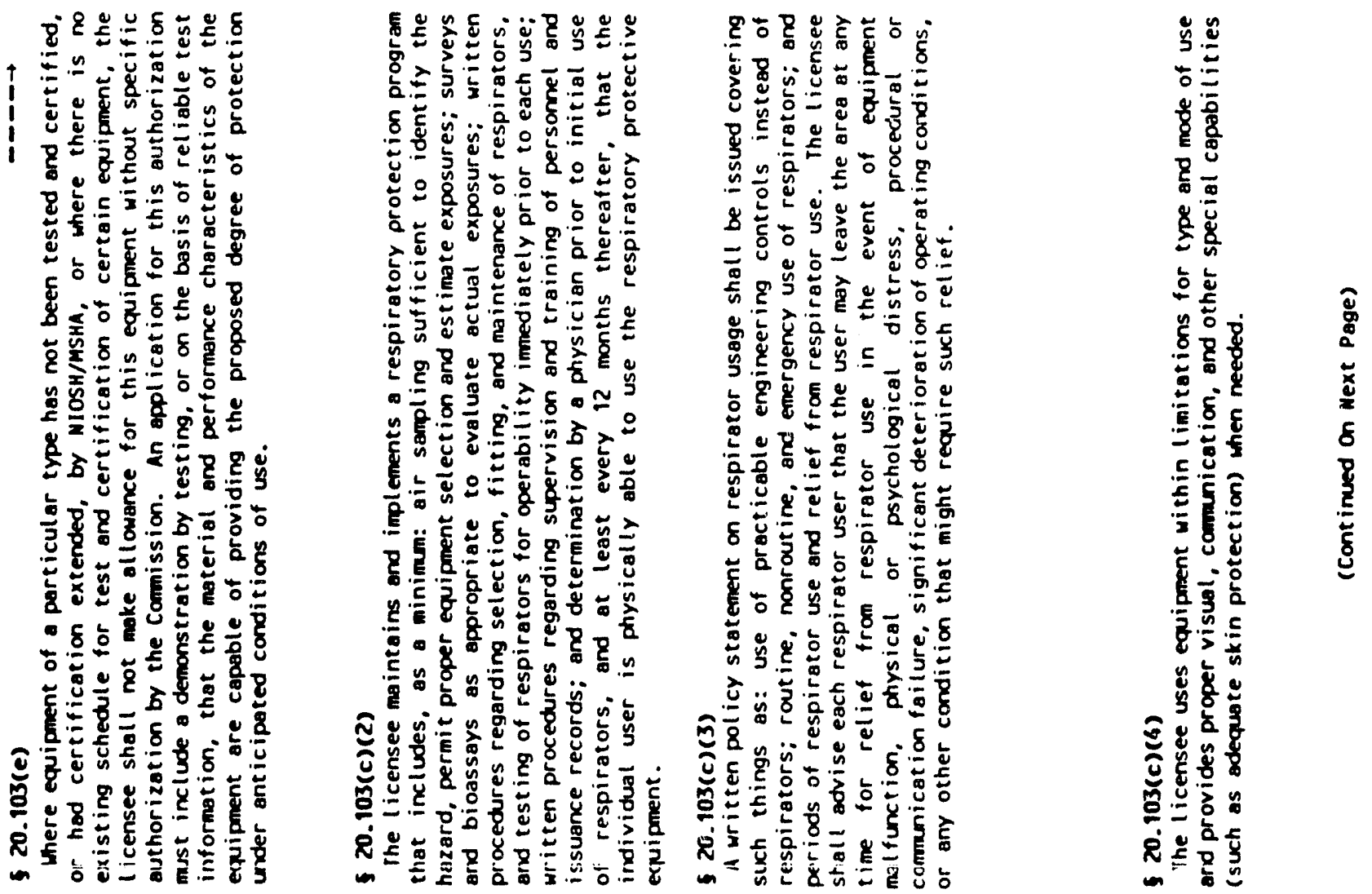


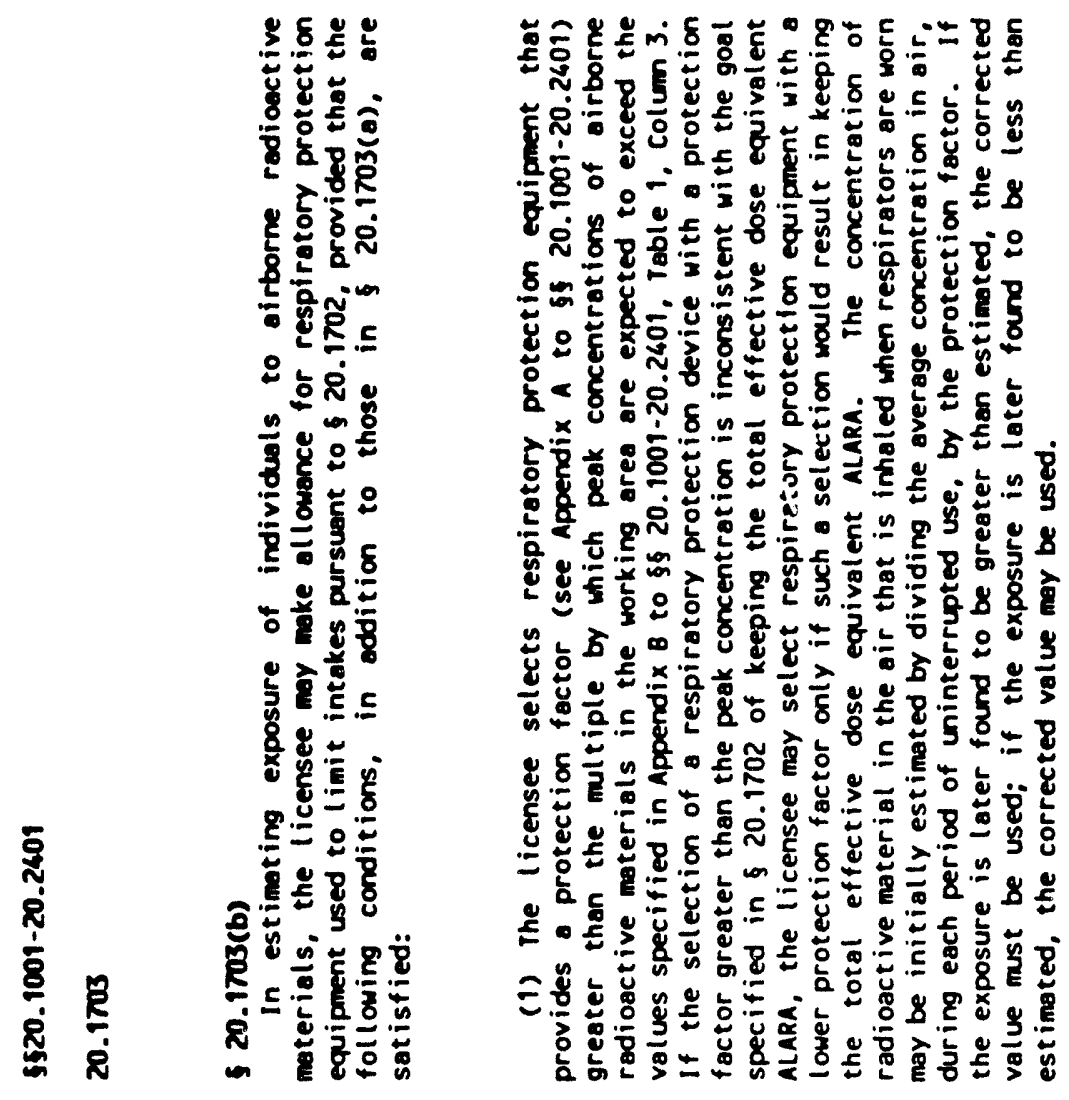

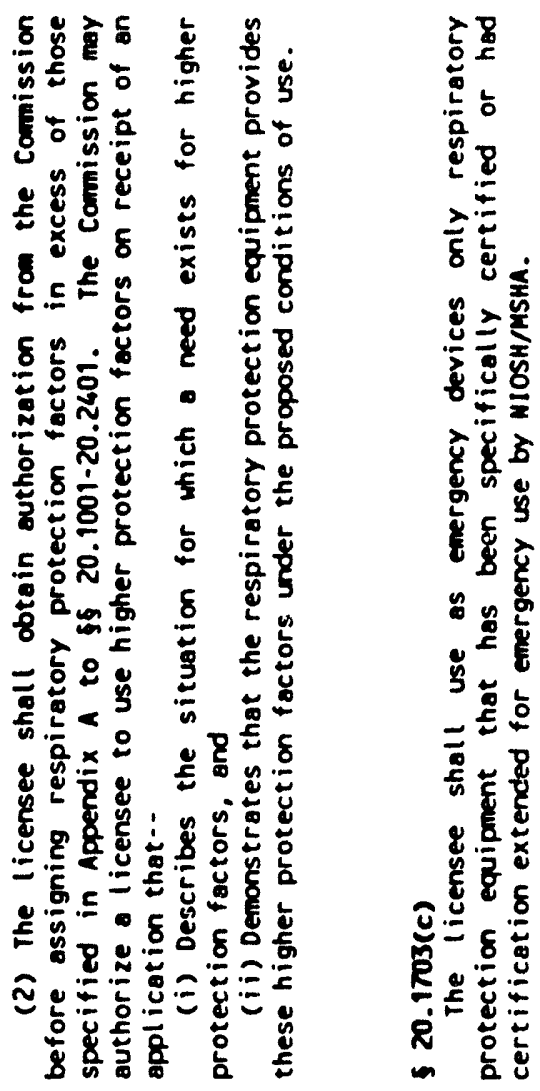



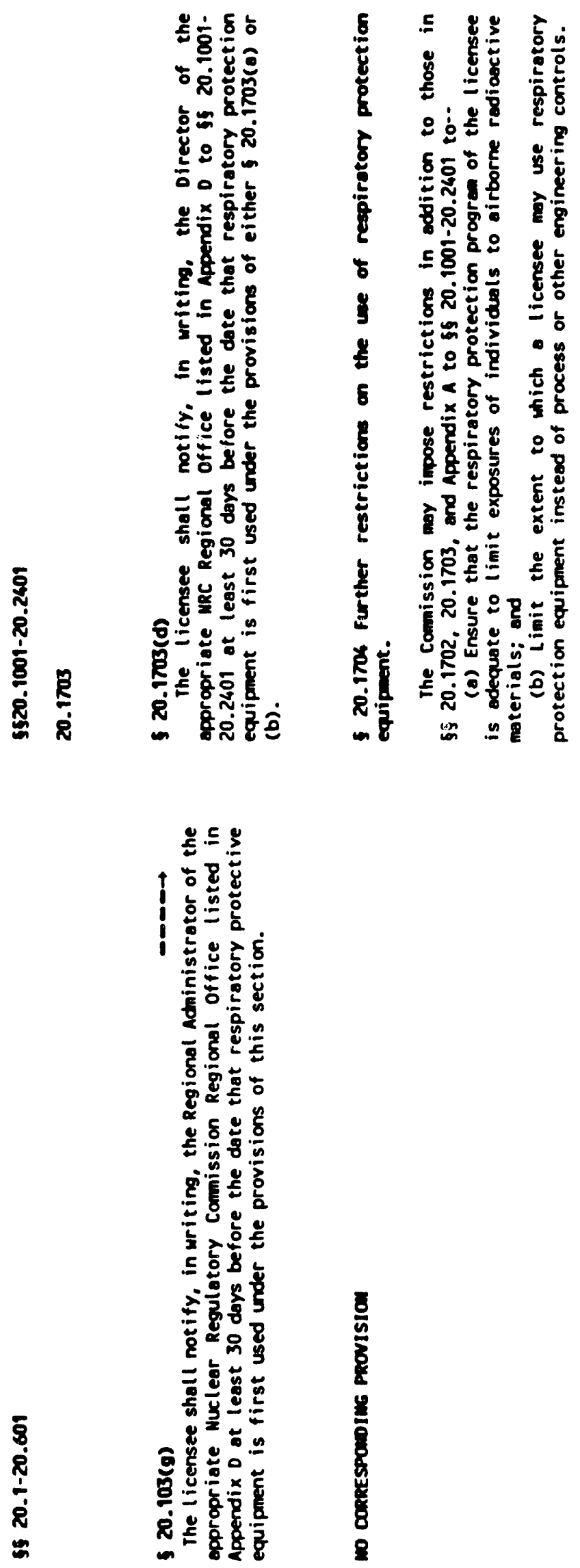


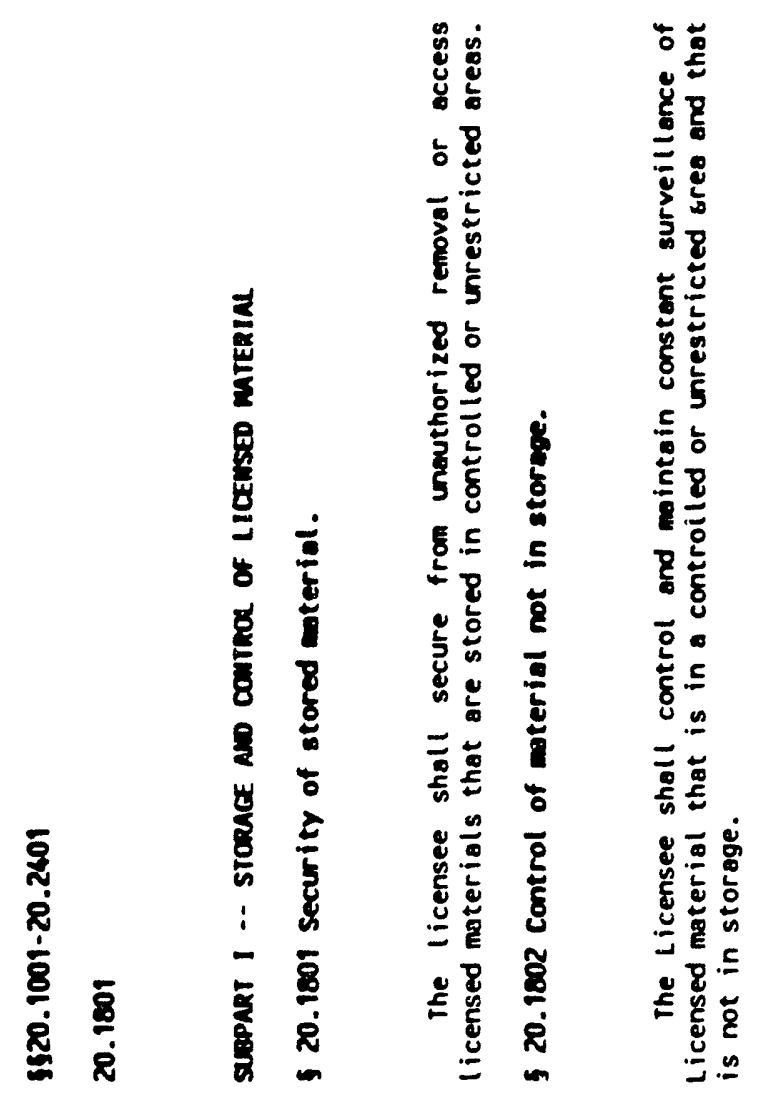

n

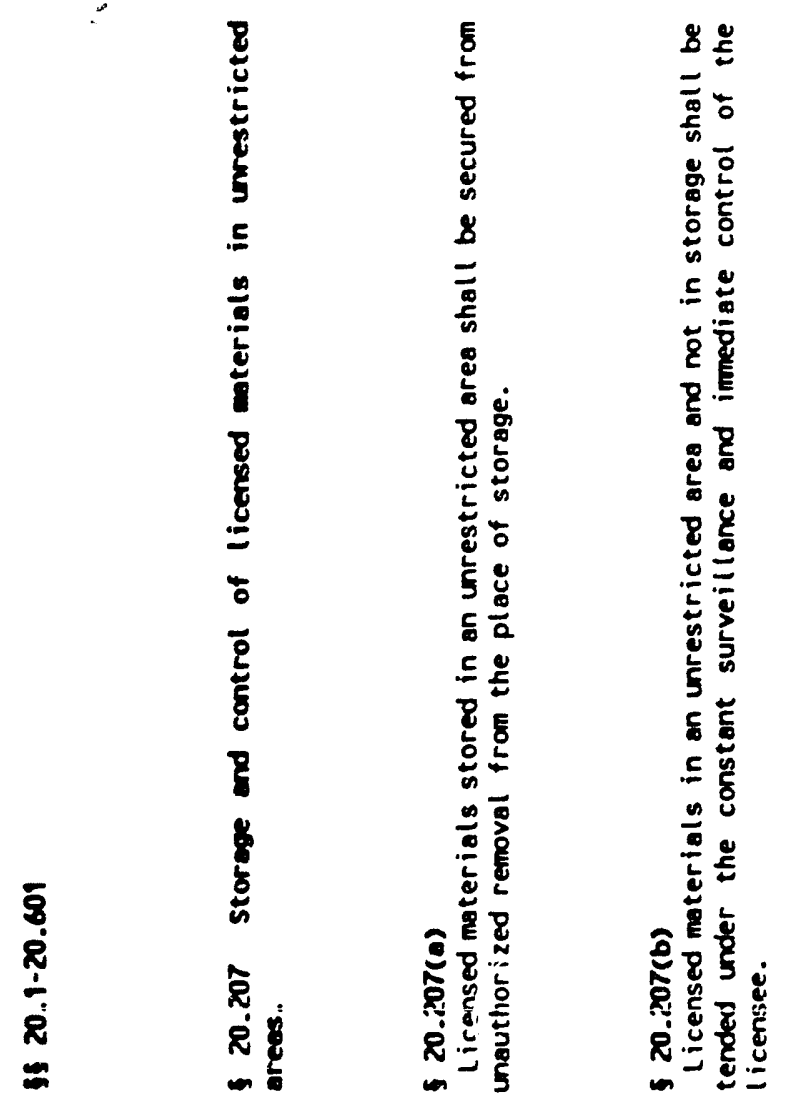




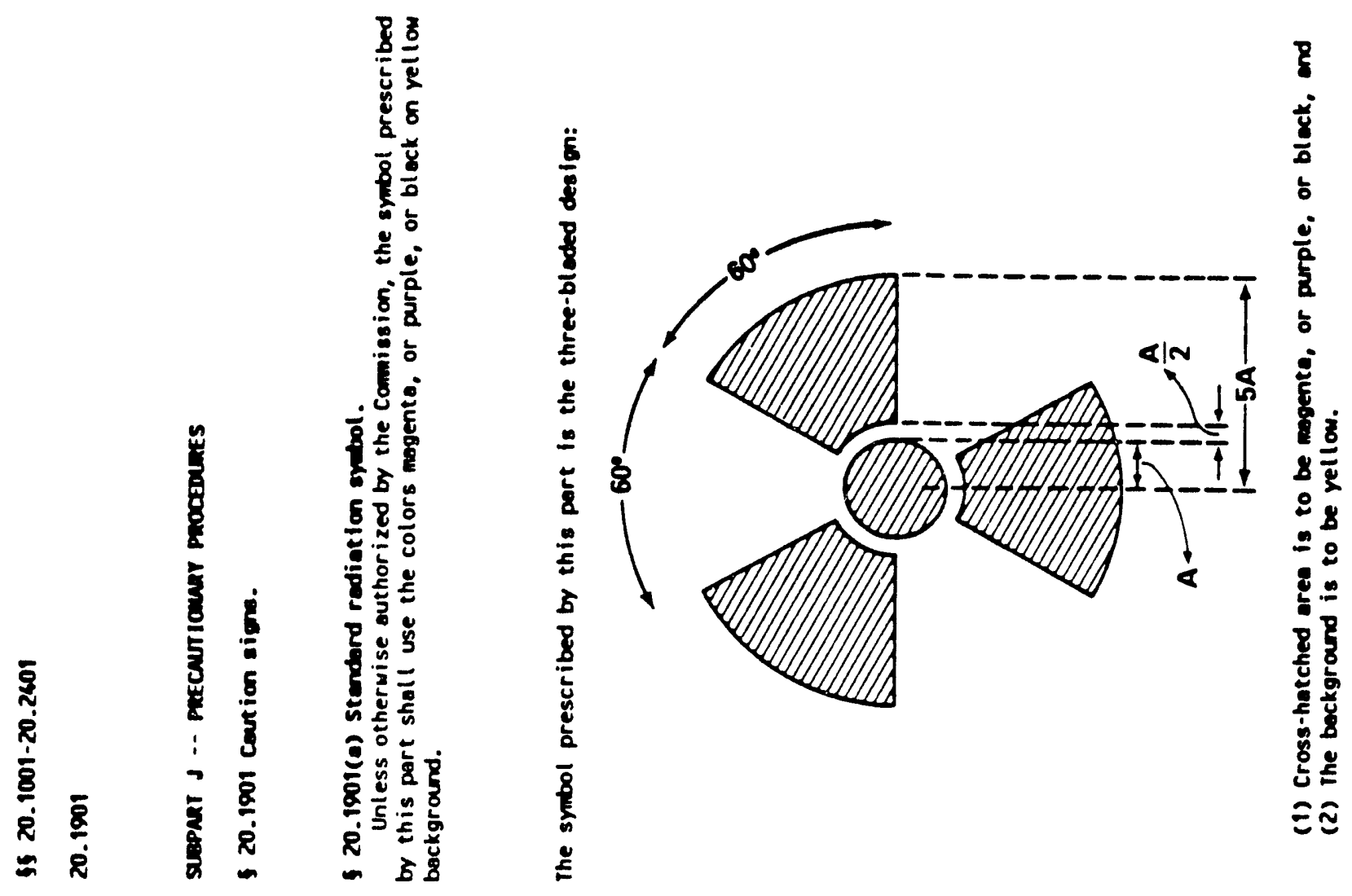

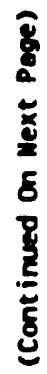

๓̊

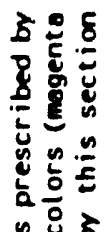

$\frac{0}{8}$
$\frac{8}{8}$
$\frac{5}{8}$
$\frac{5}{8}$

85

के

它

实

造造

它它客

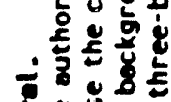

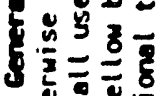

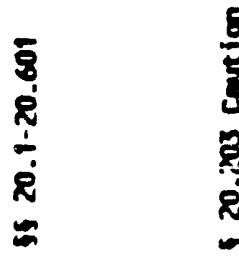

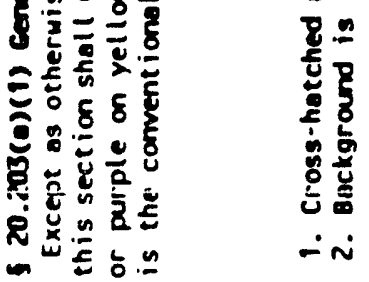

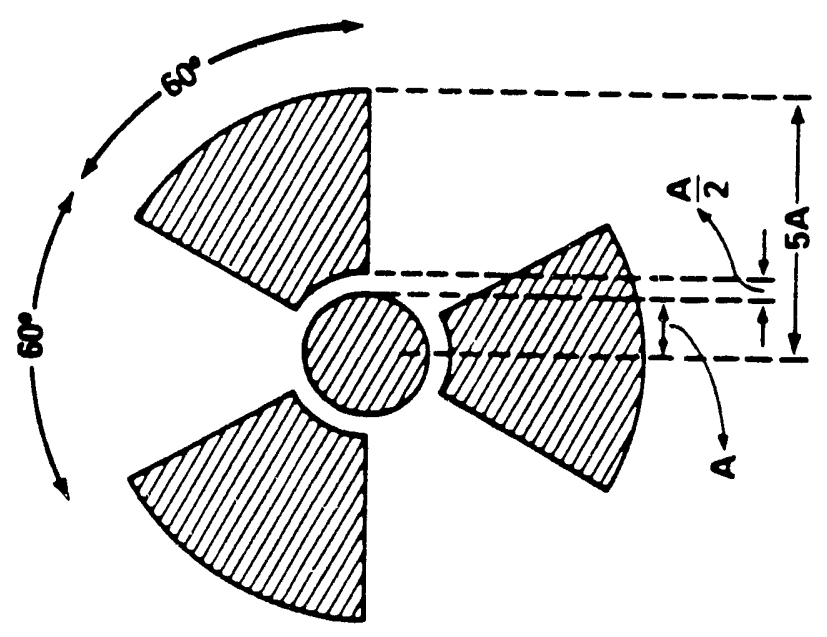

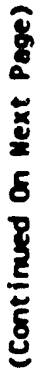



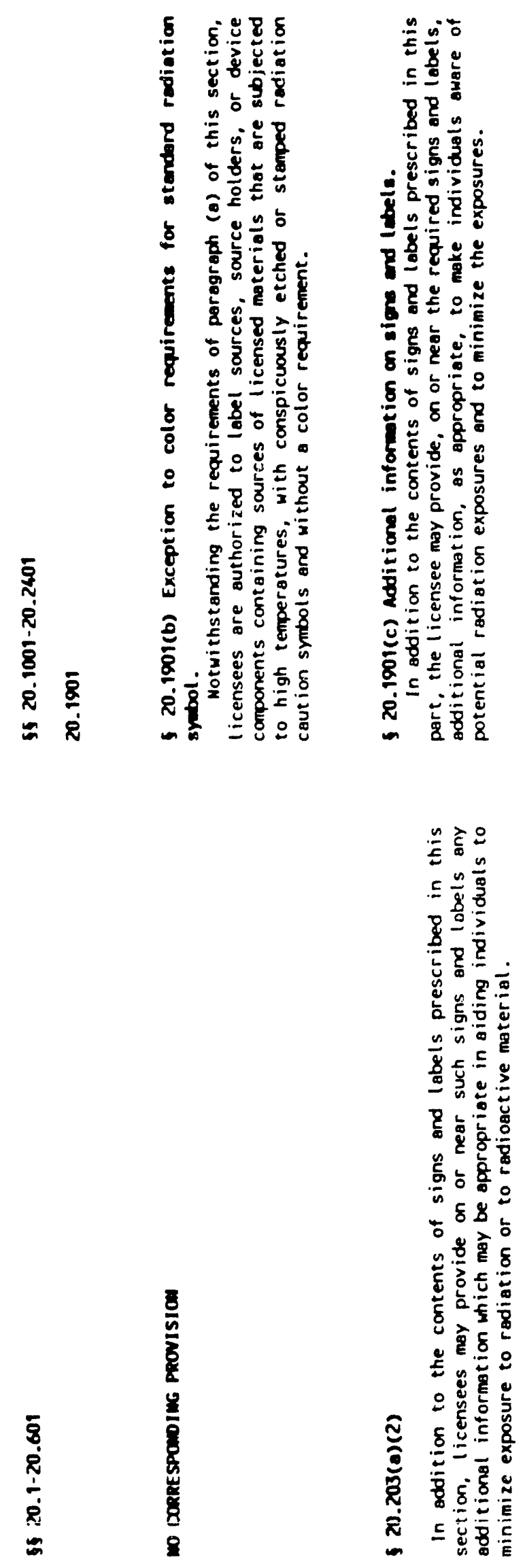

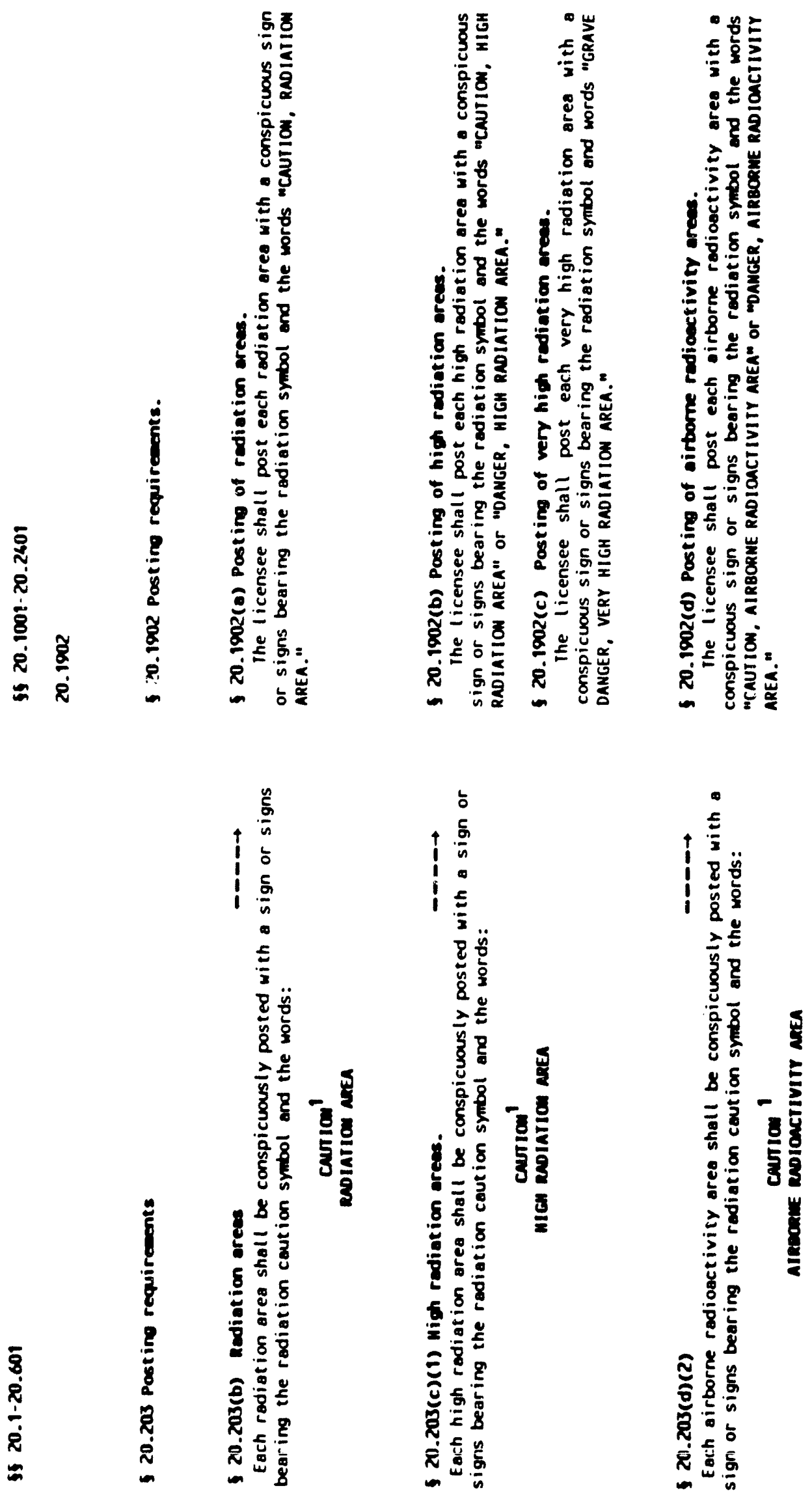

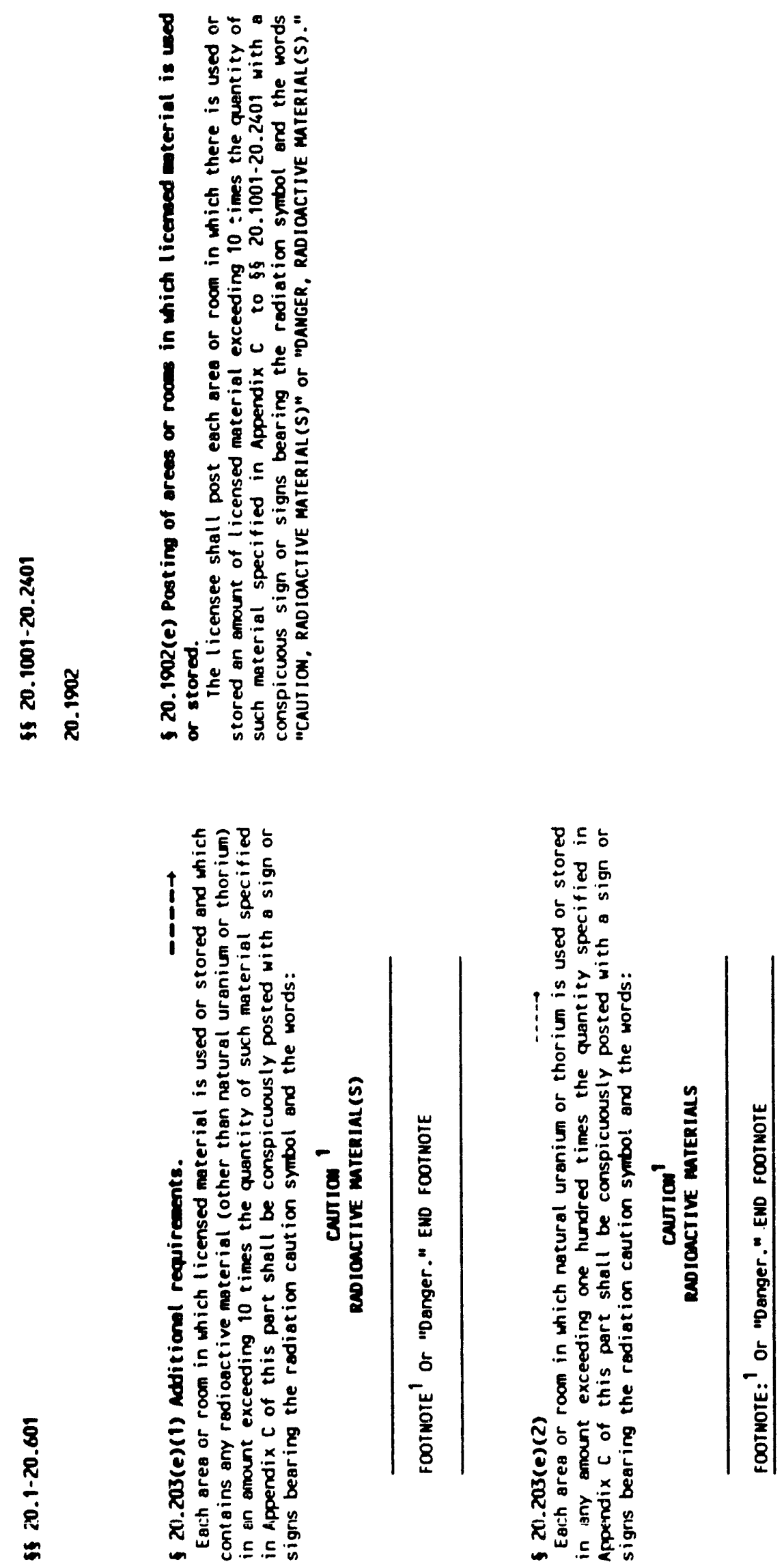

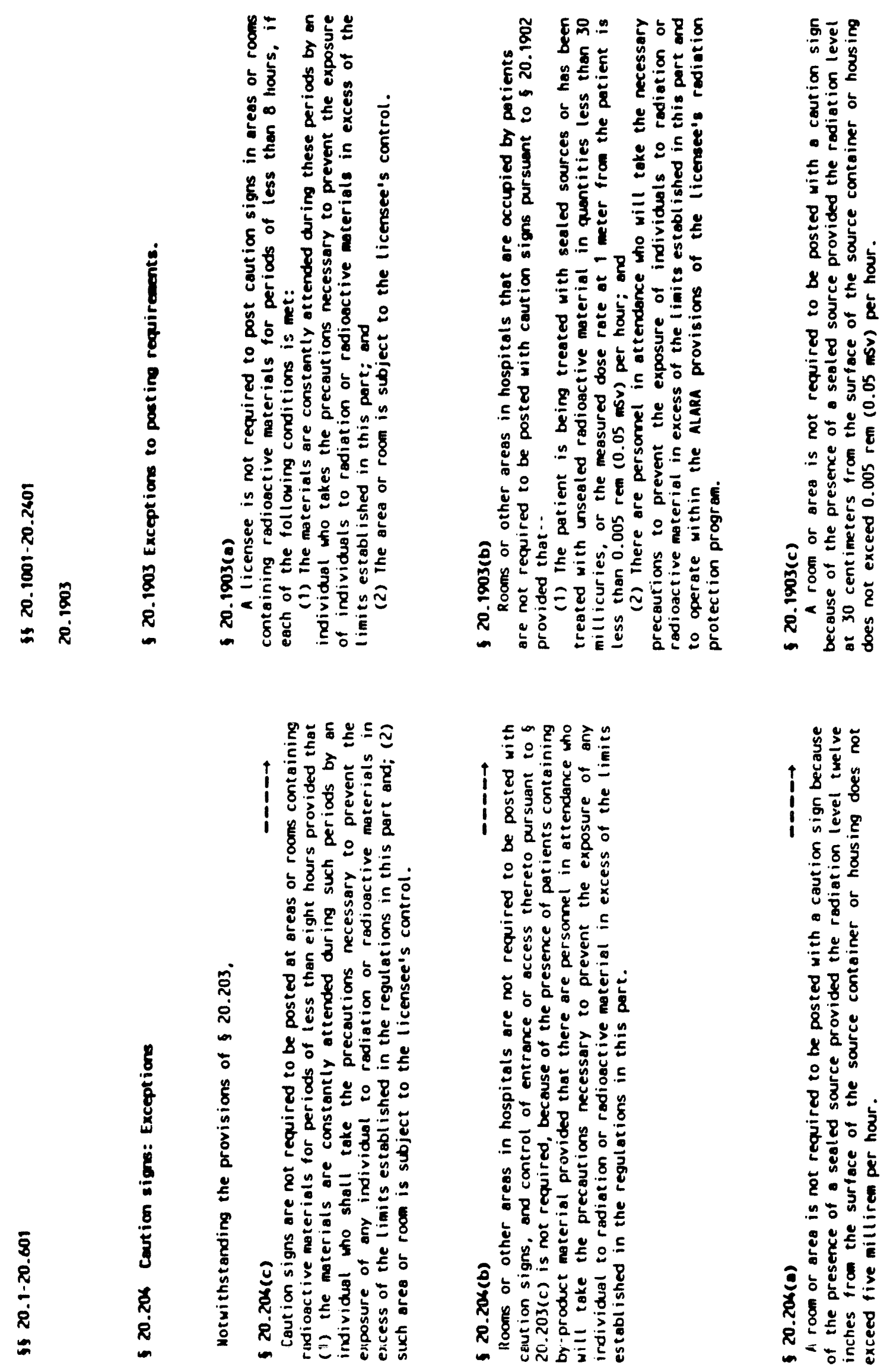

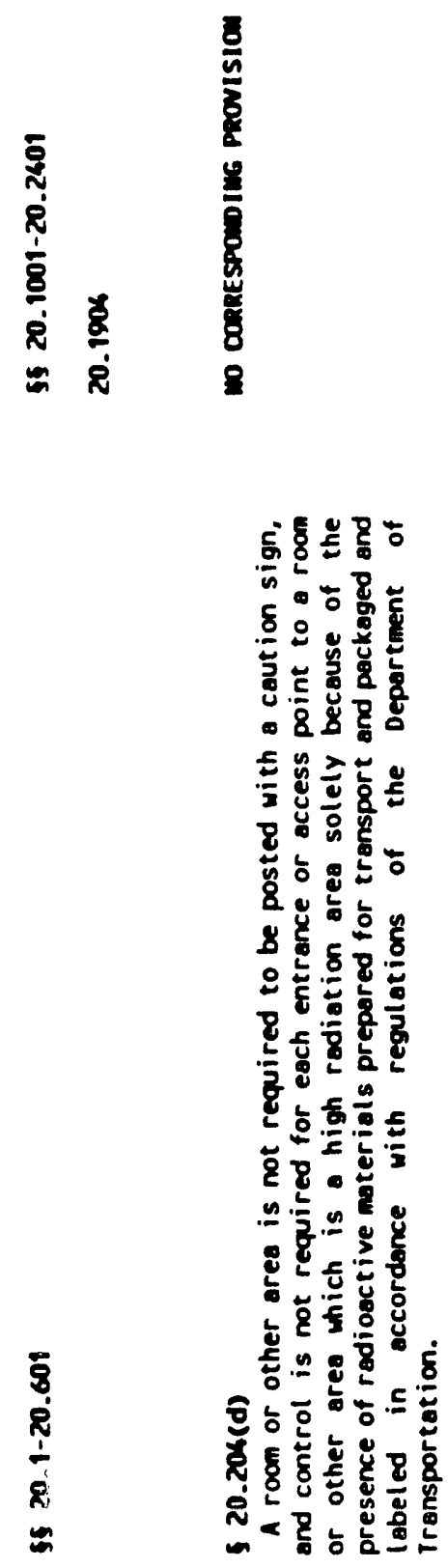

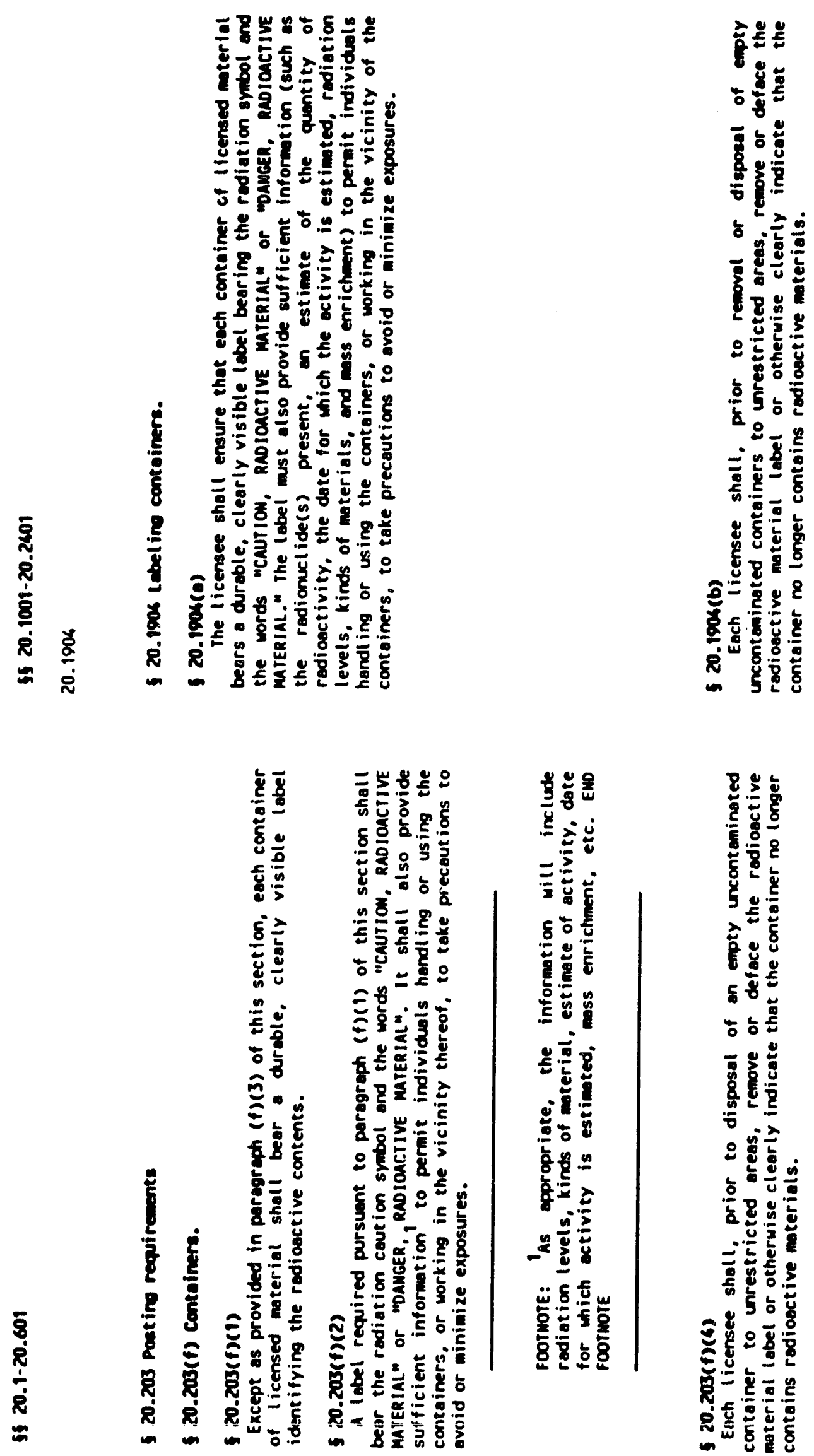


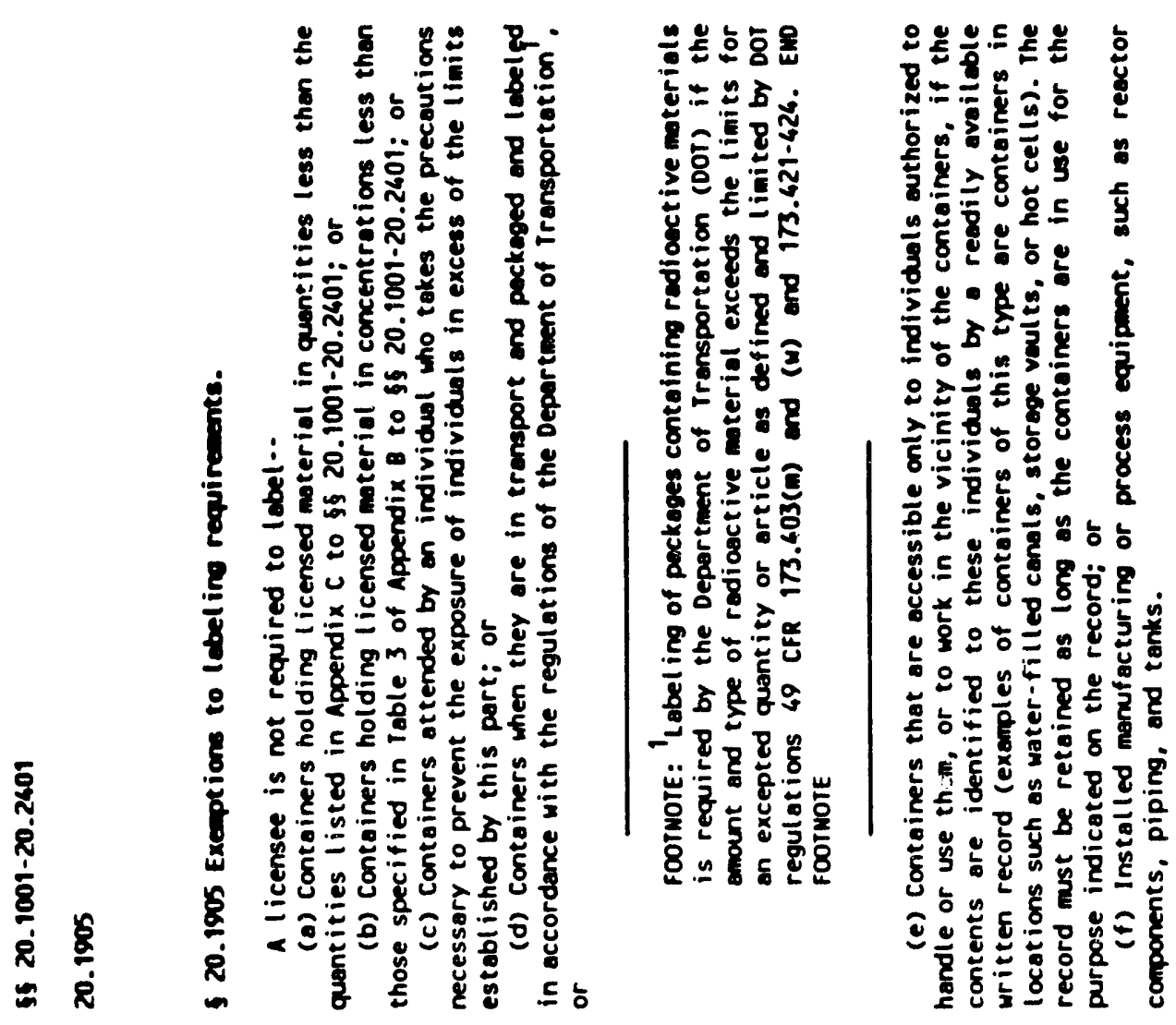



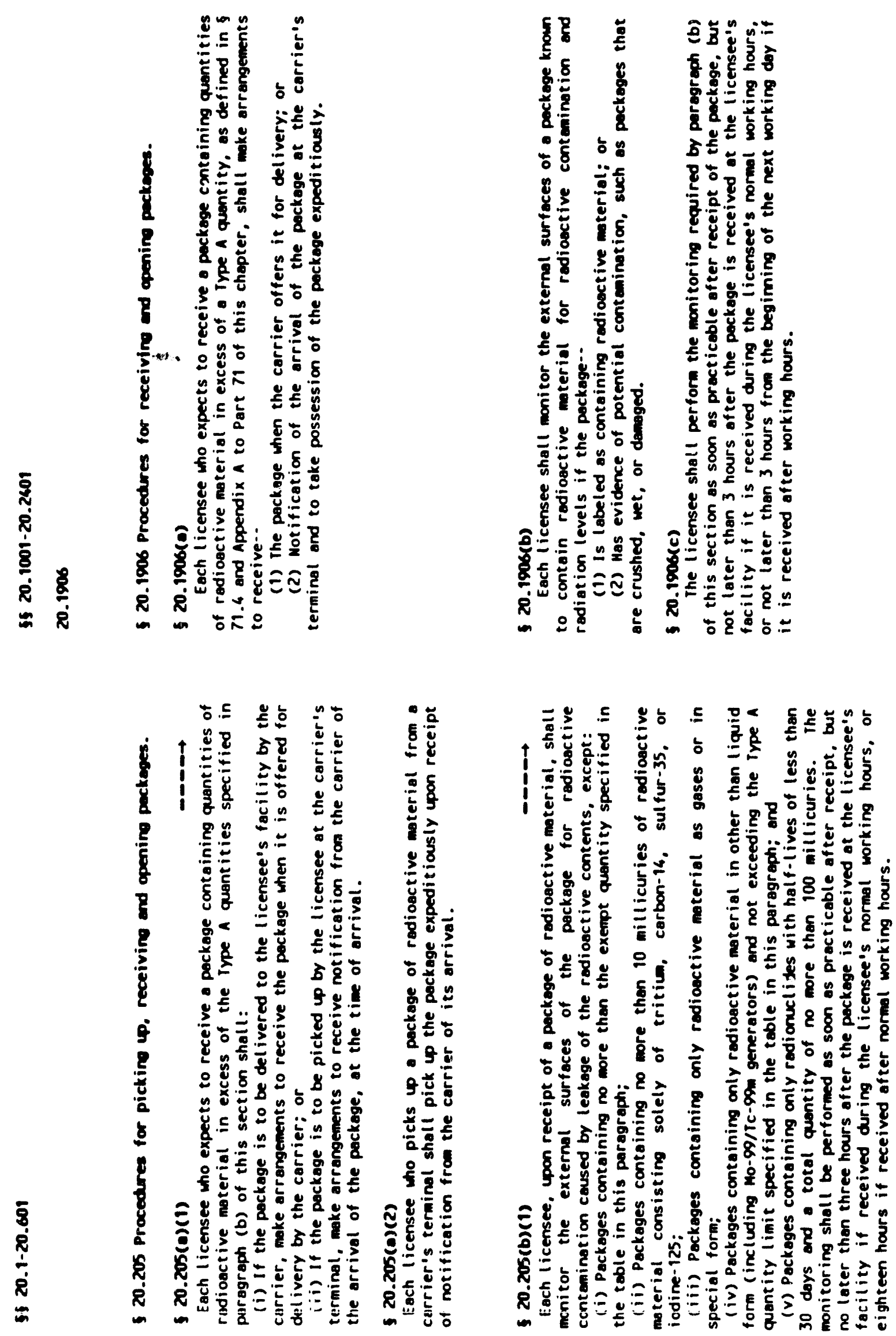

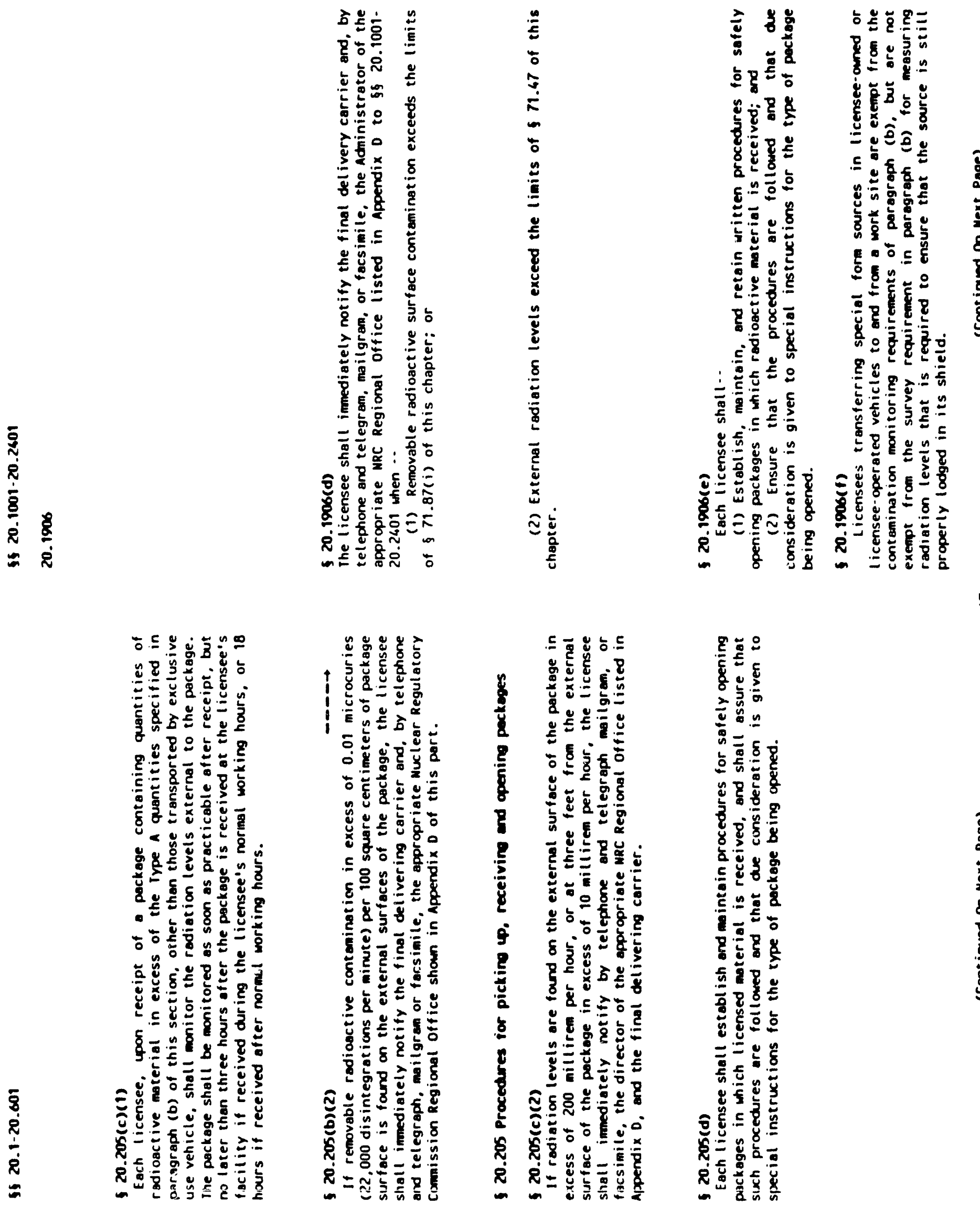

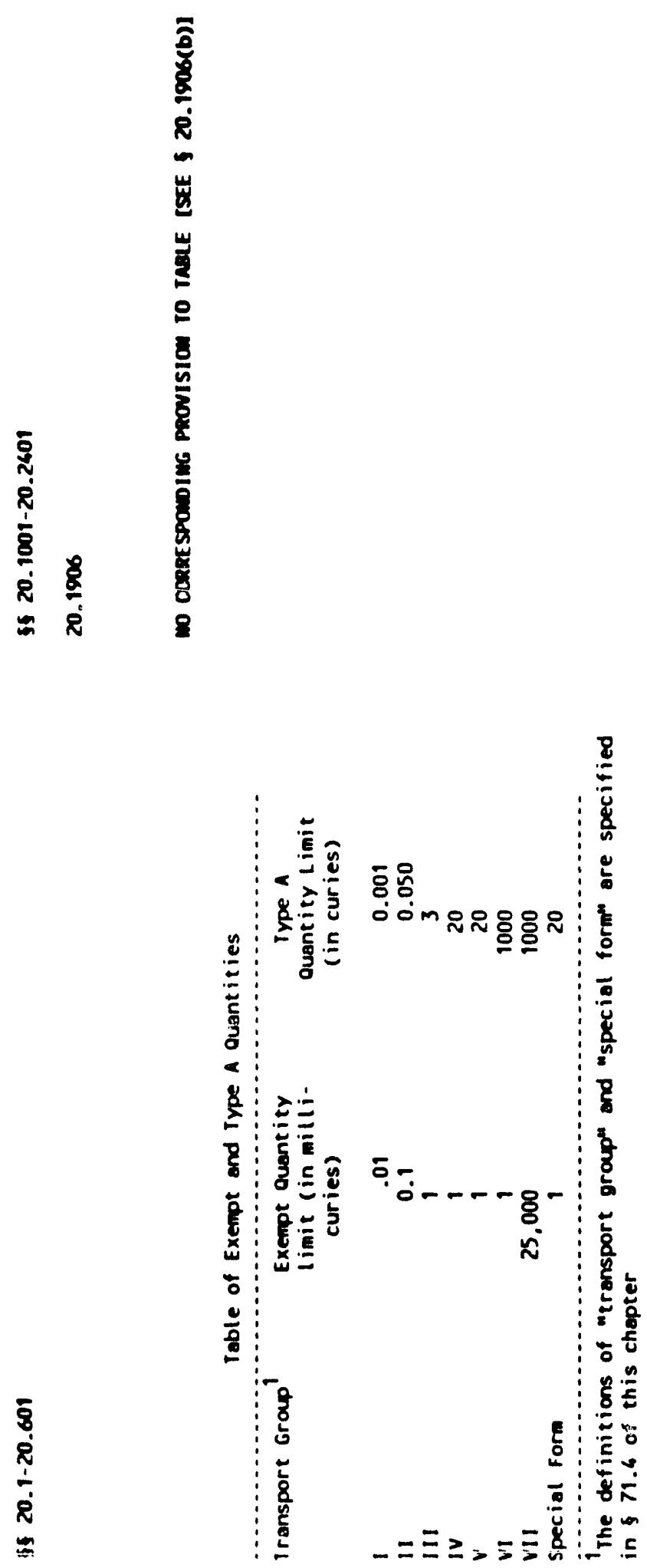

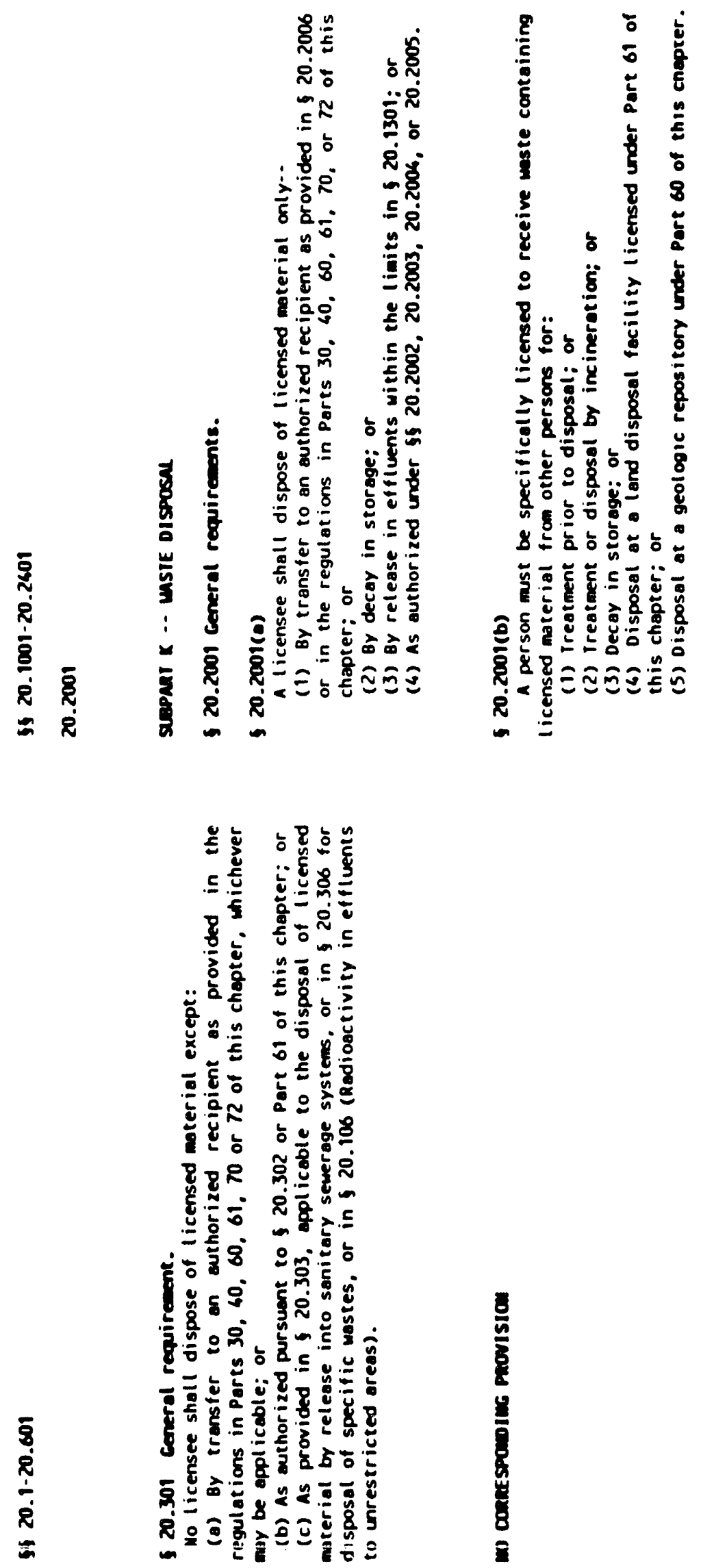

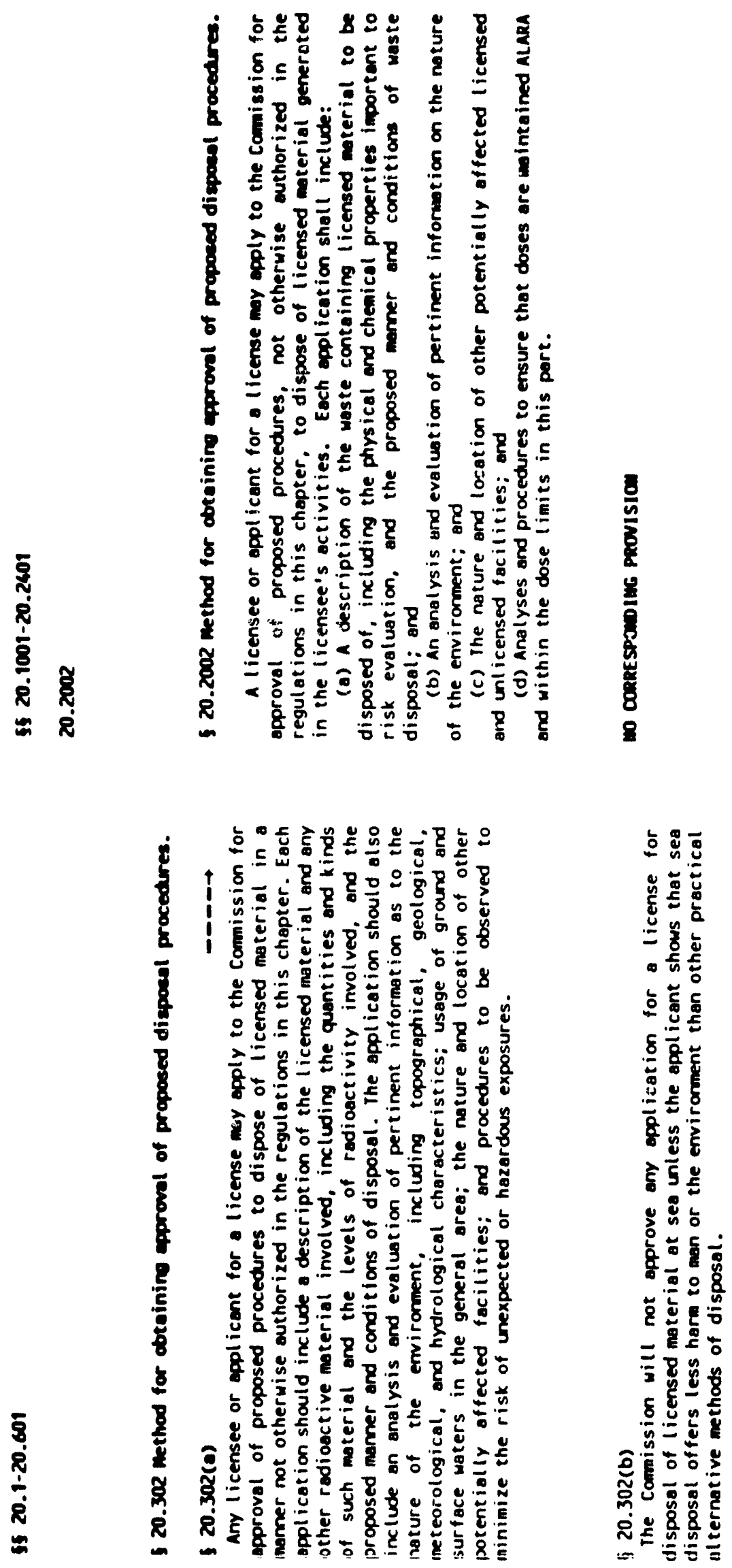

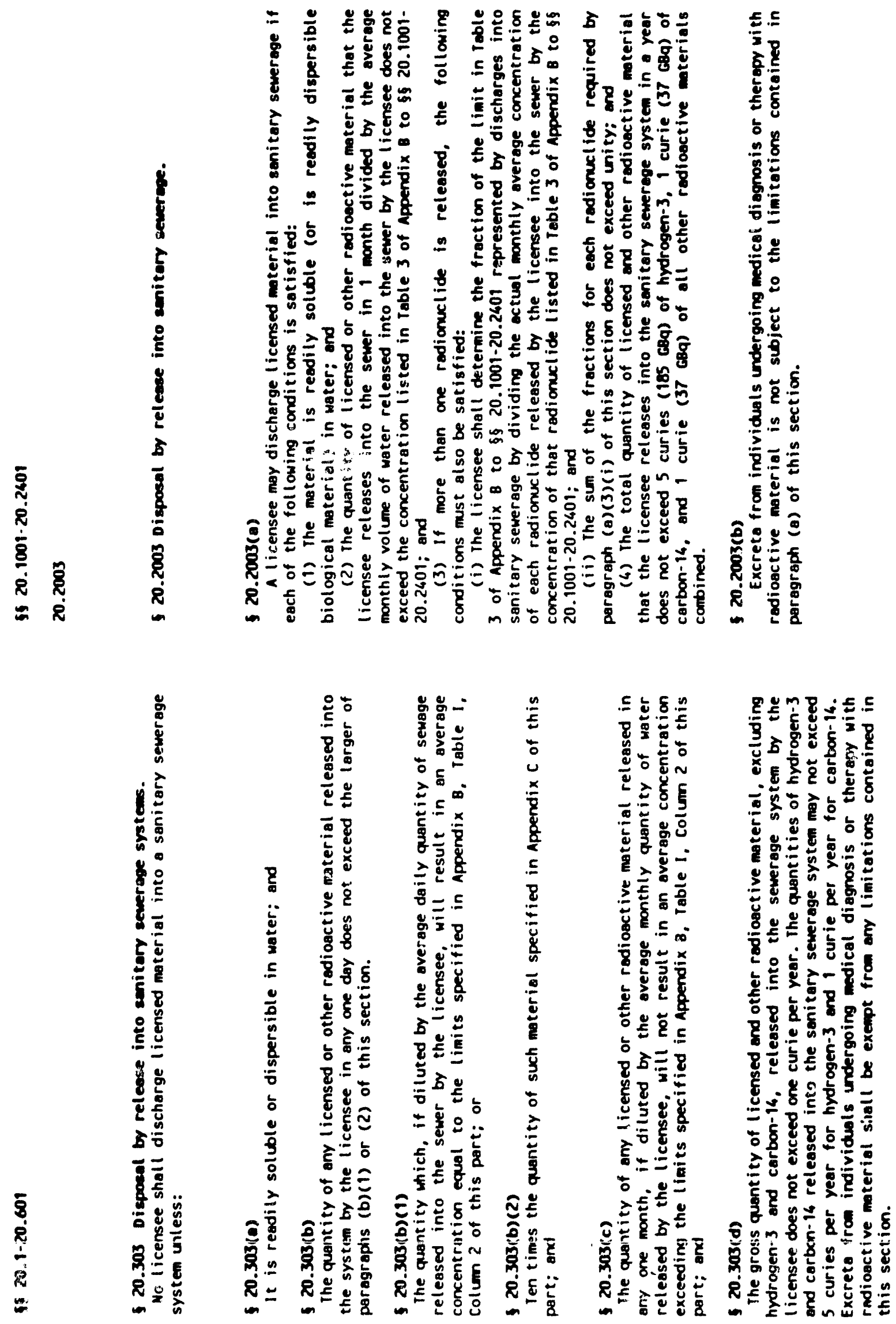


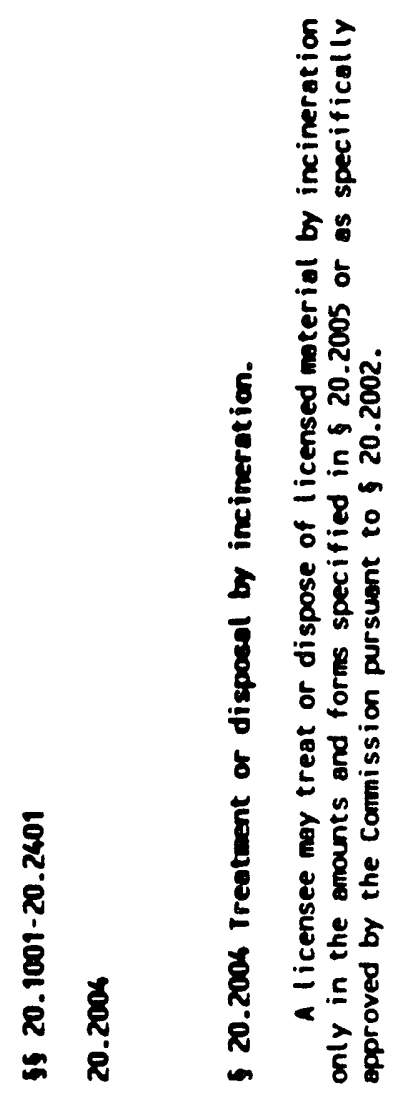

N

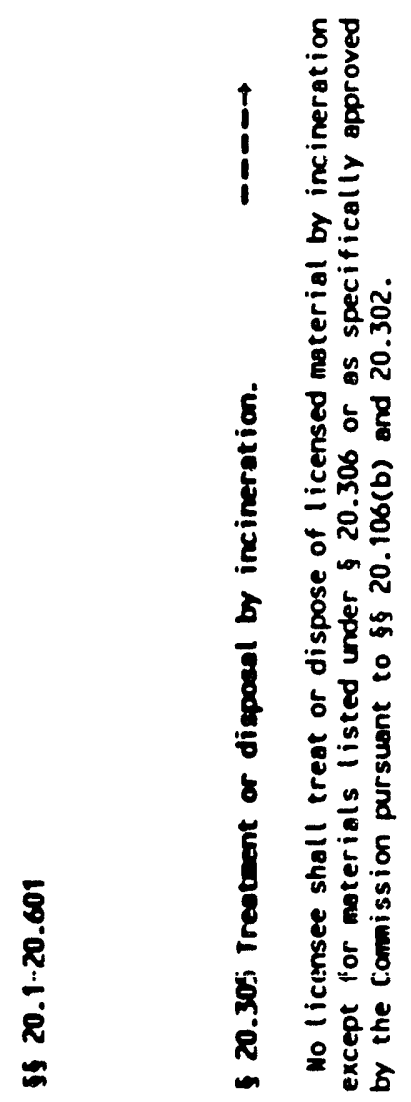



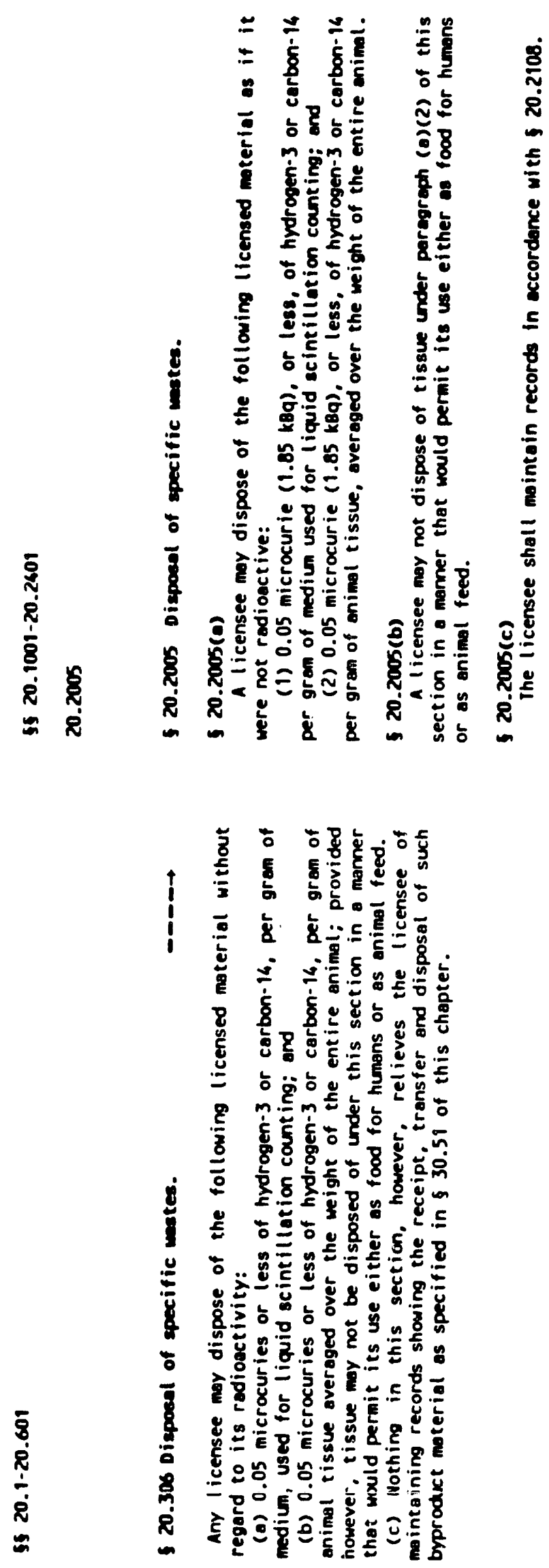

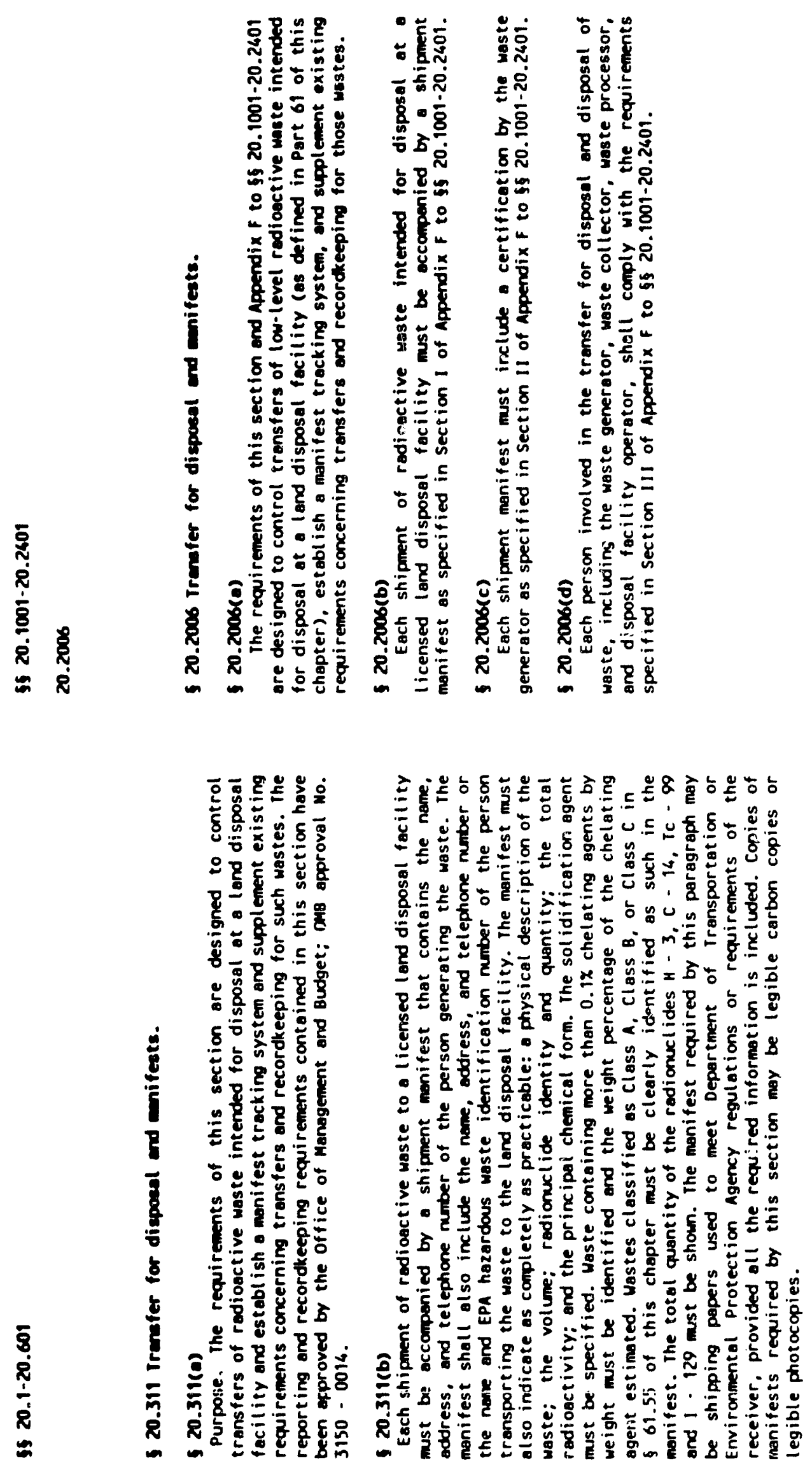

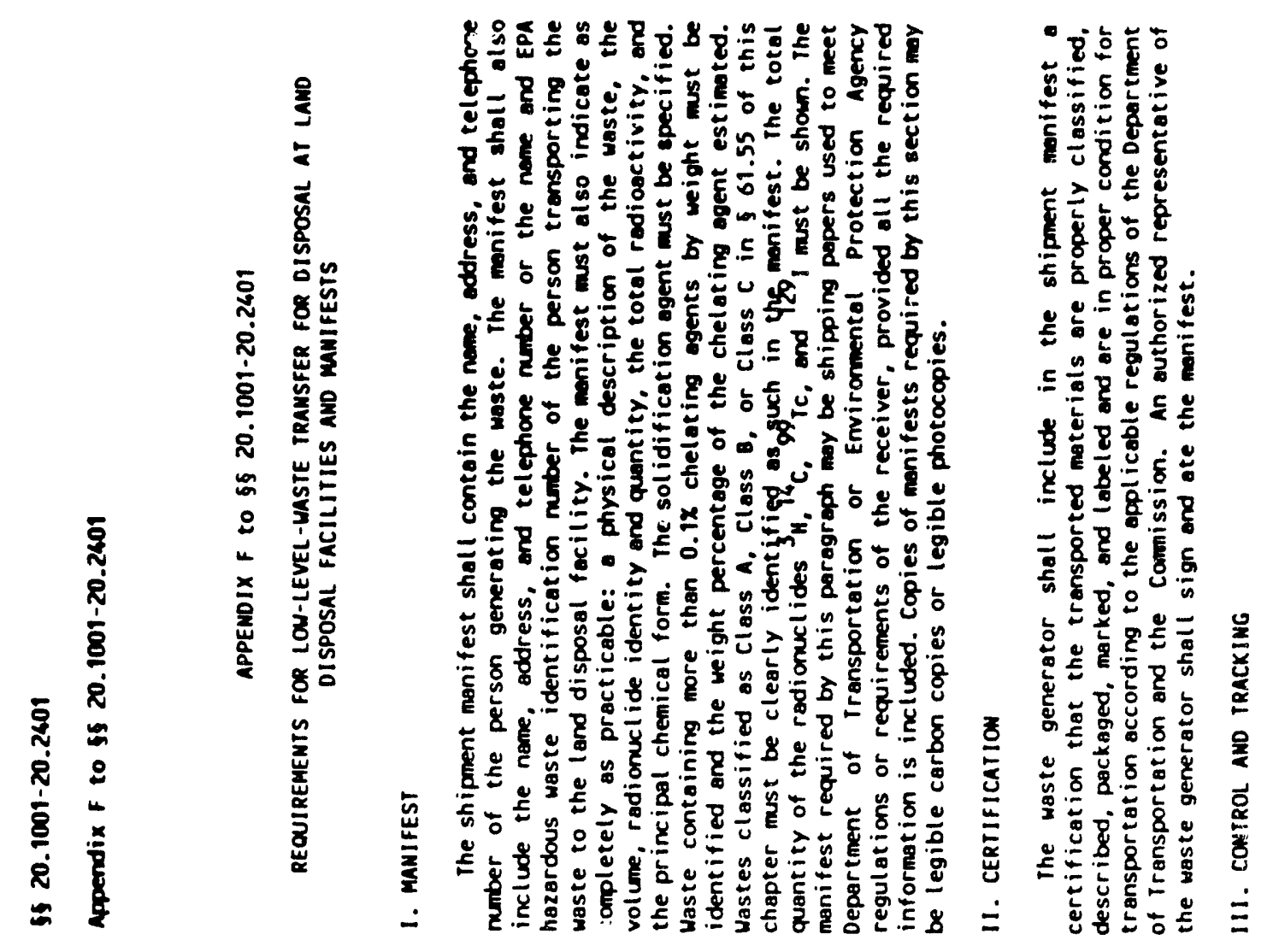

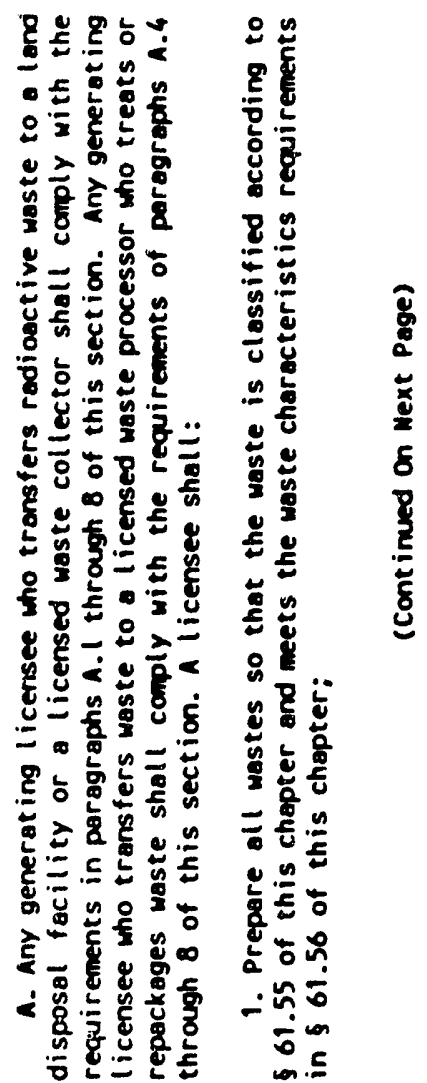

$K$

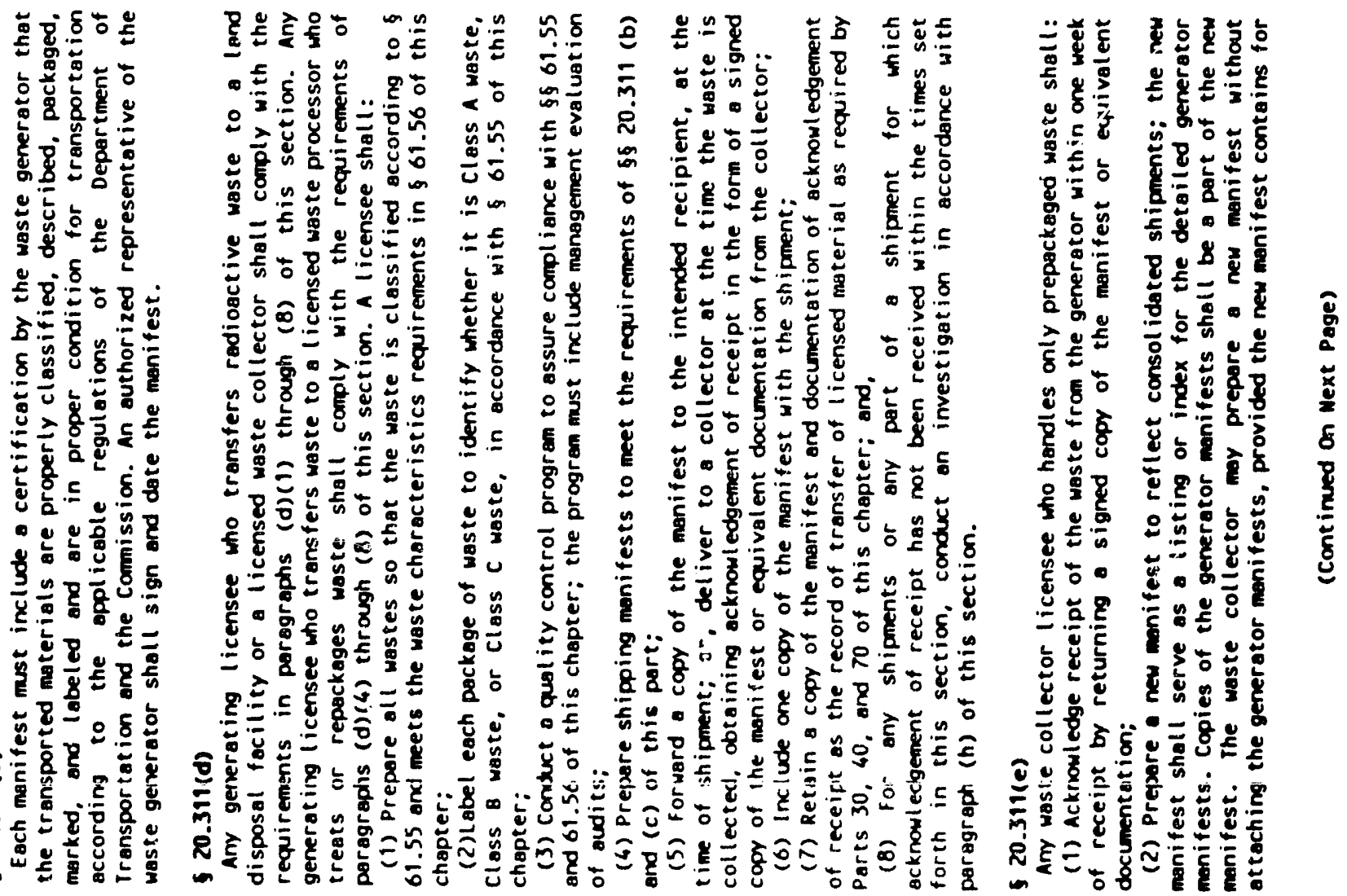




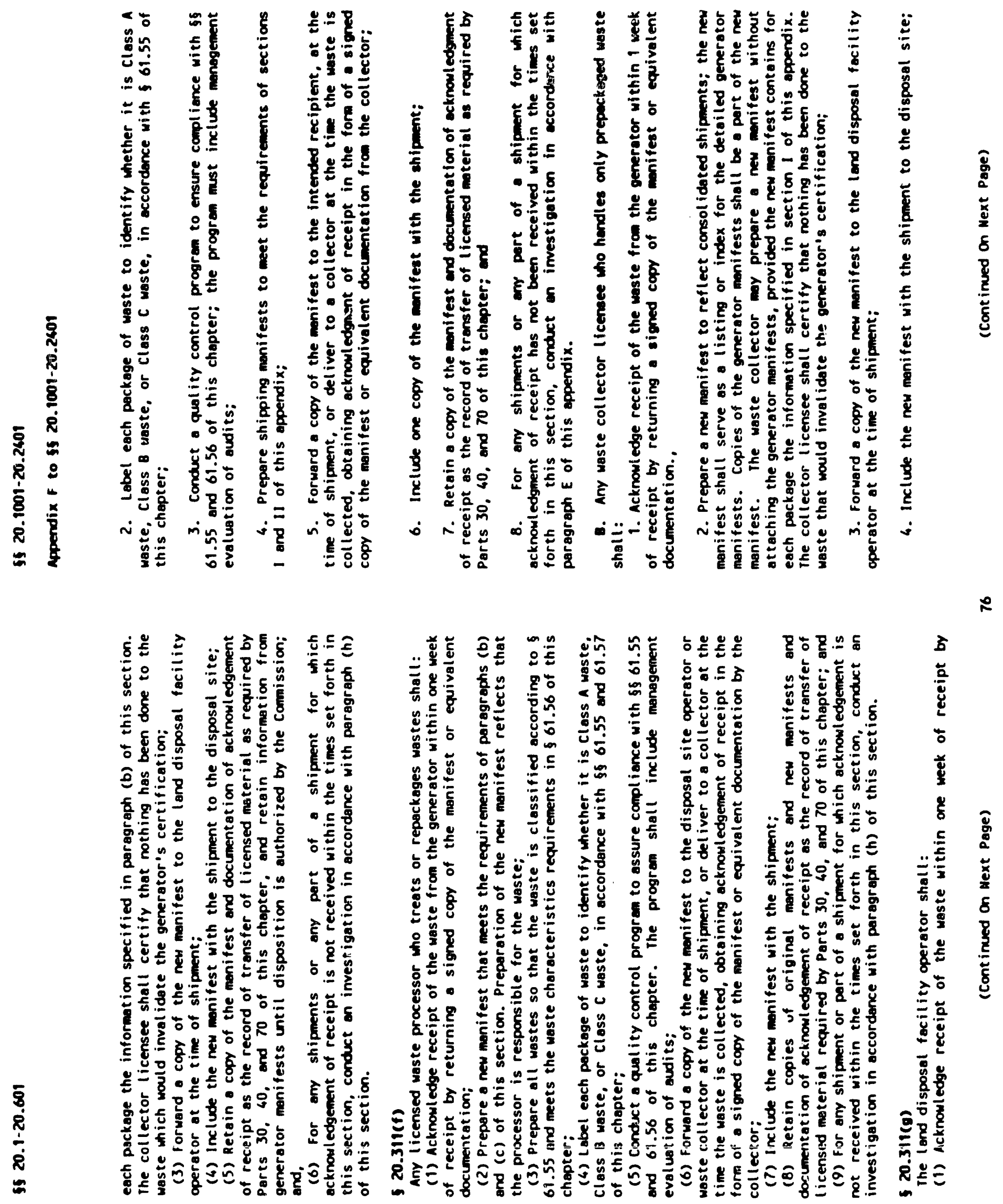




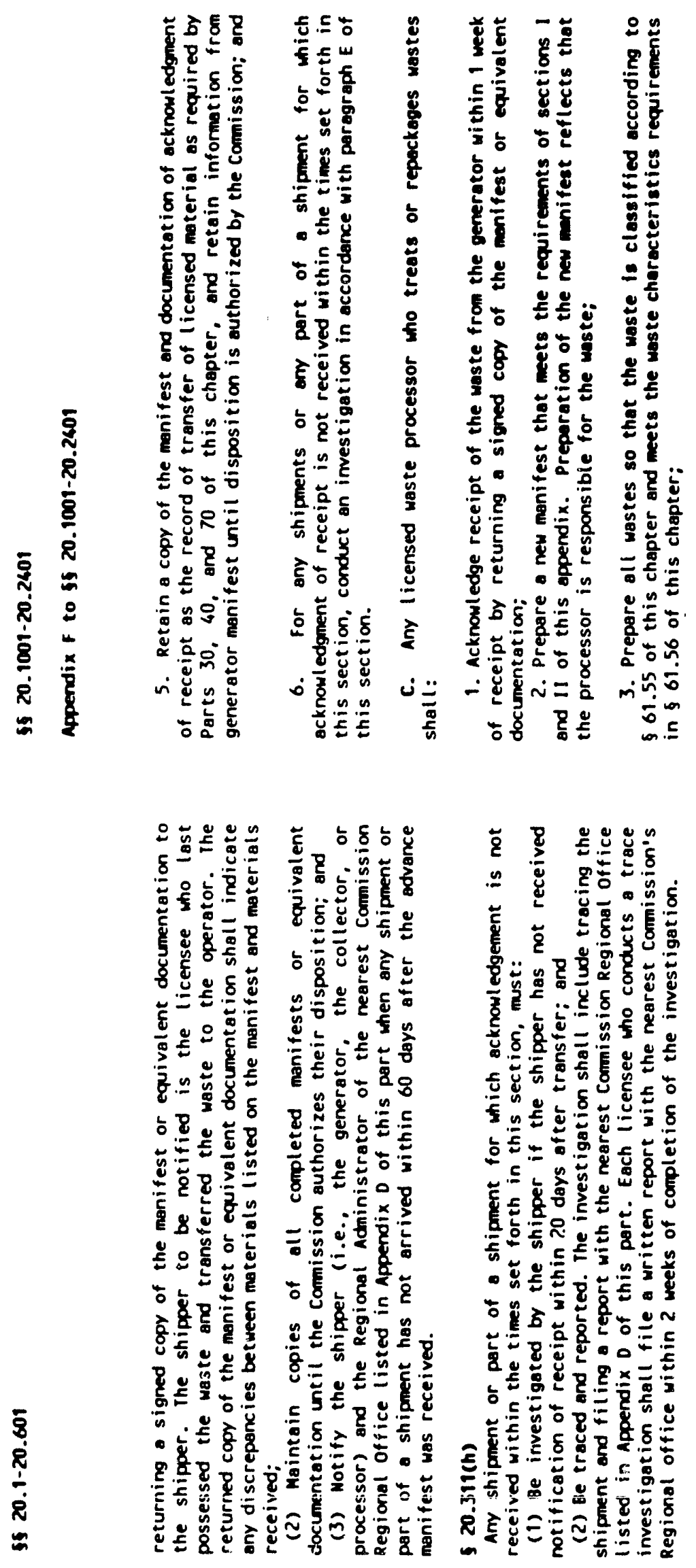




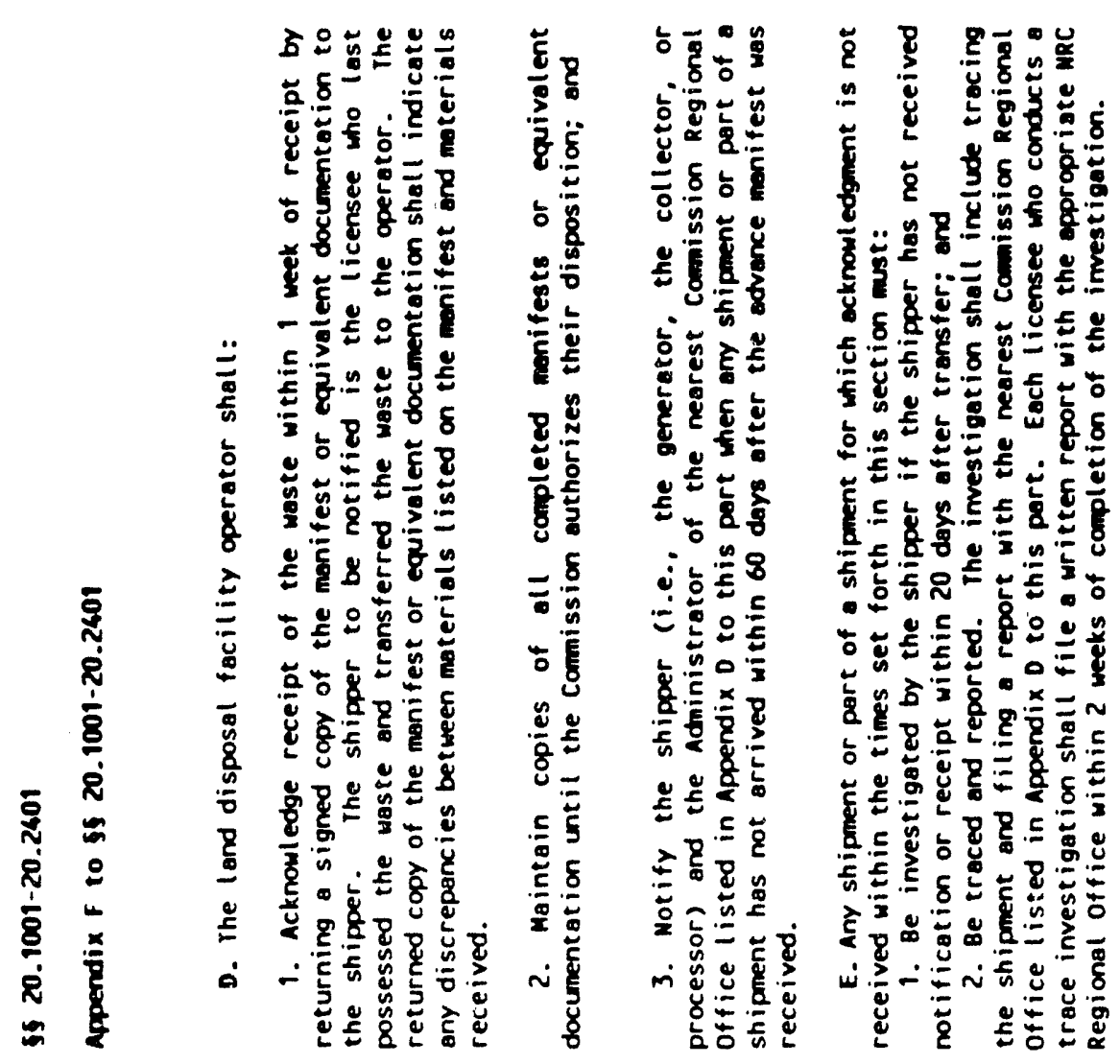

 


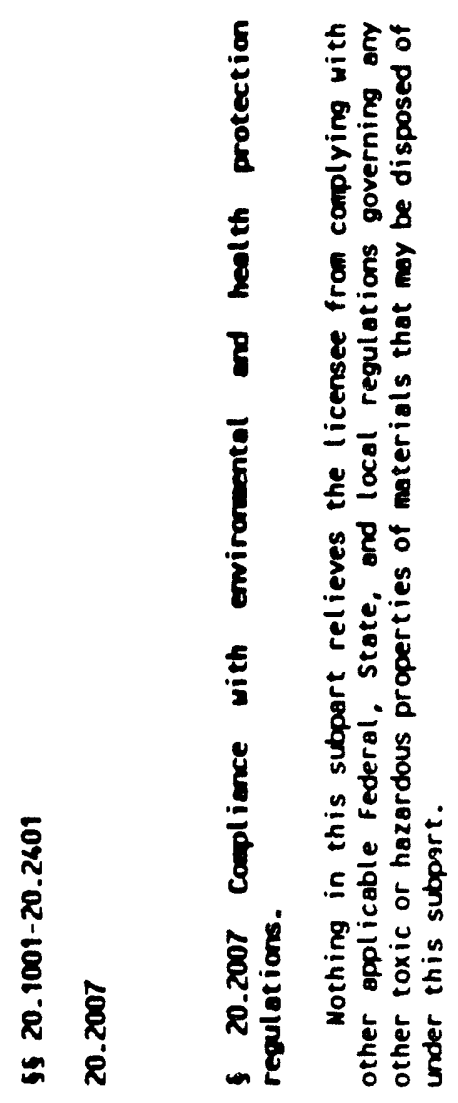

$R$

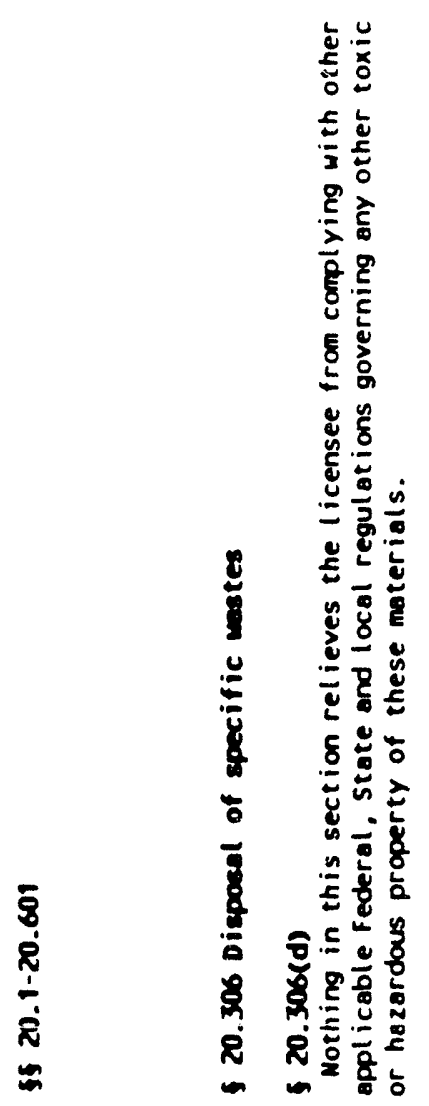



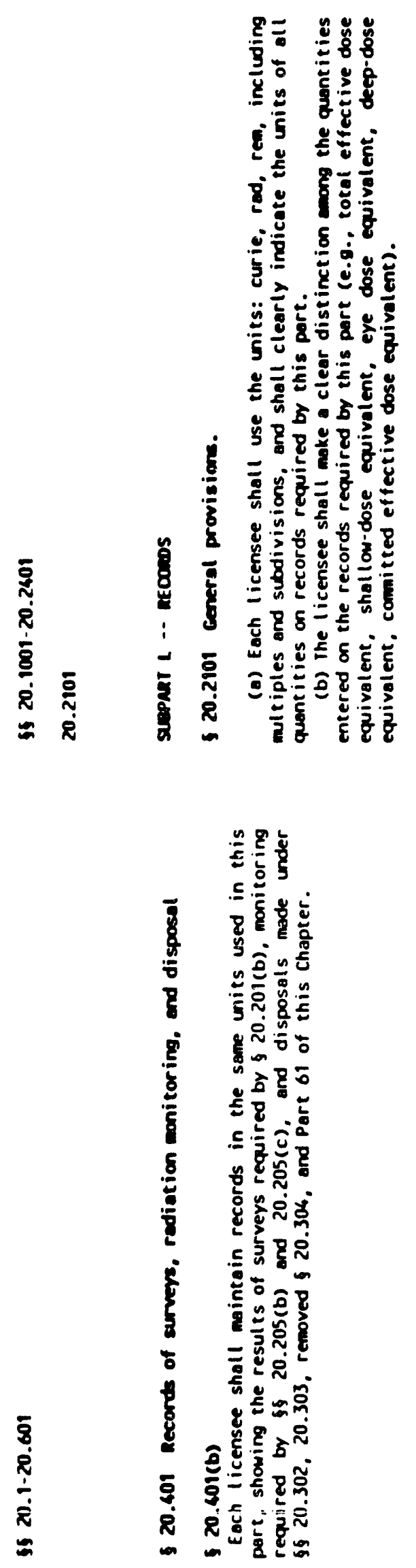

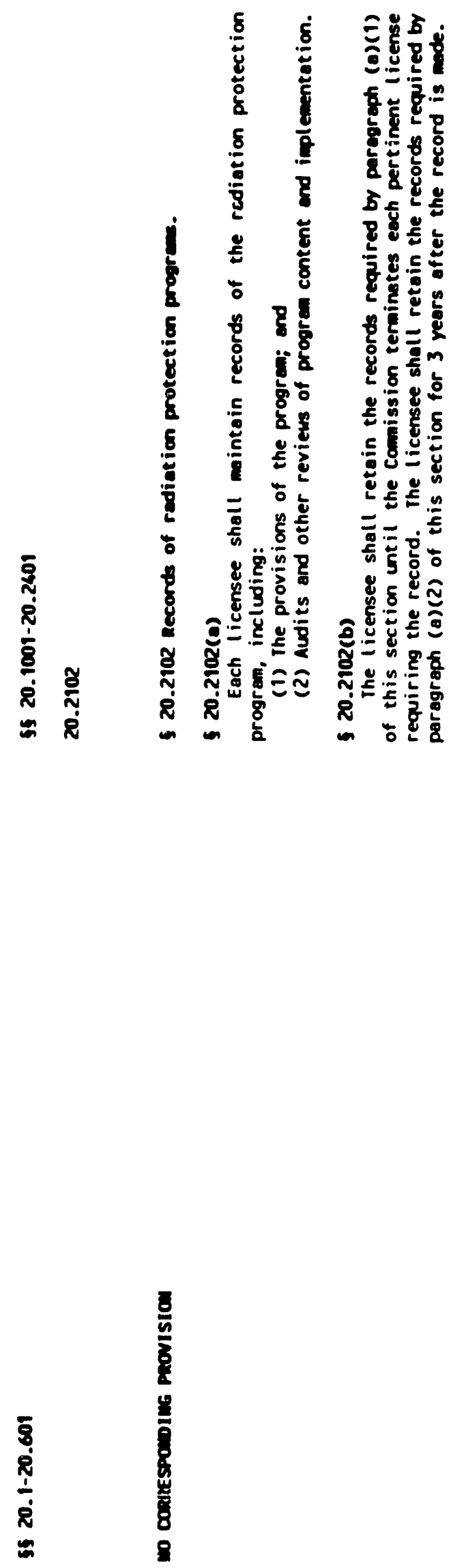


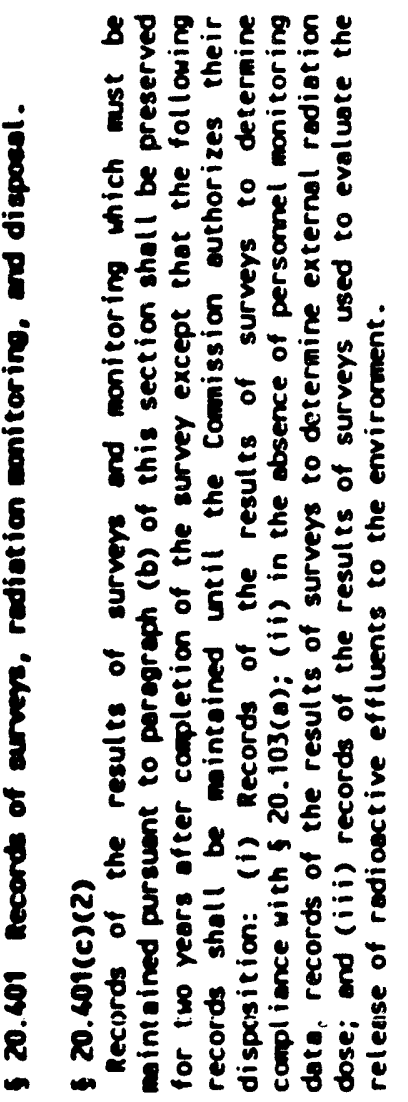




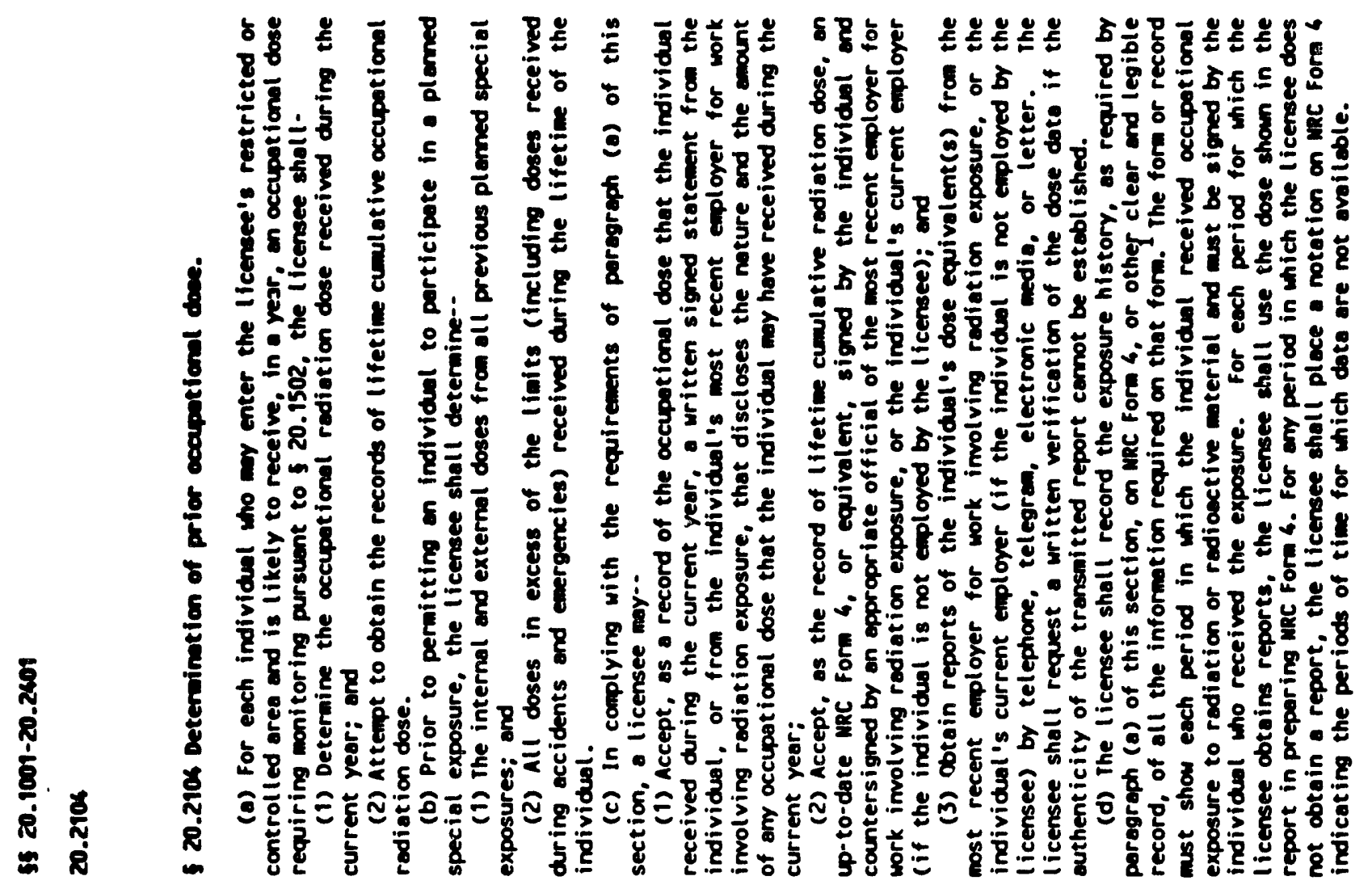

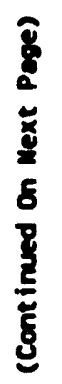

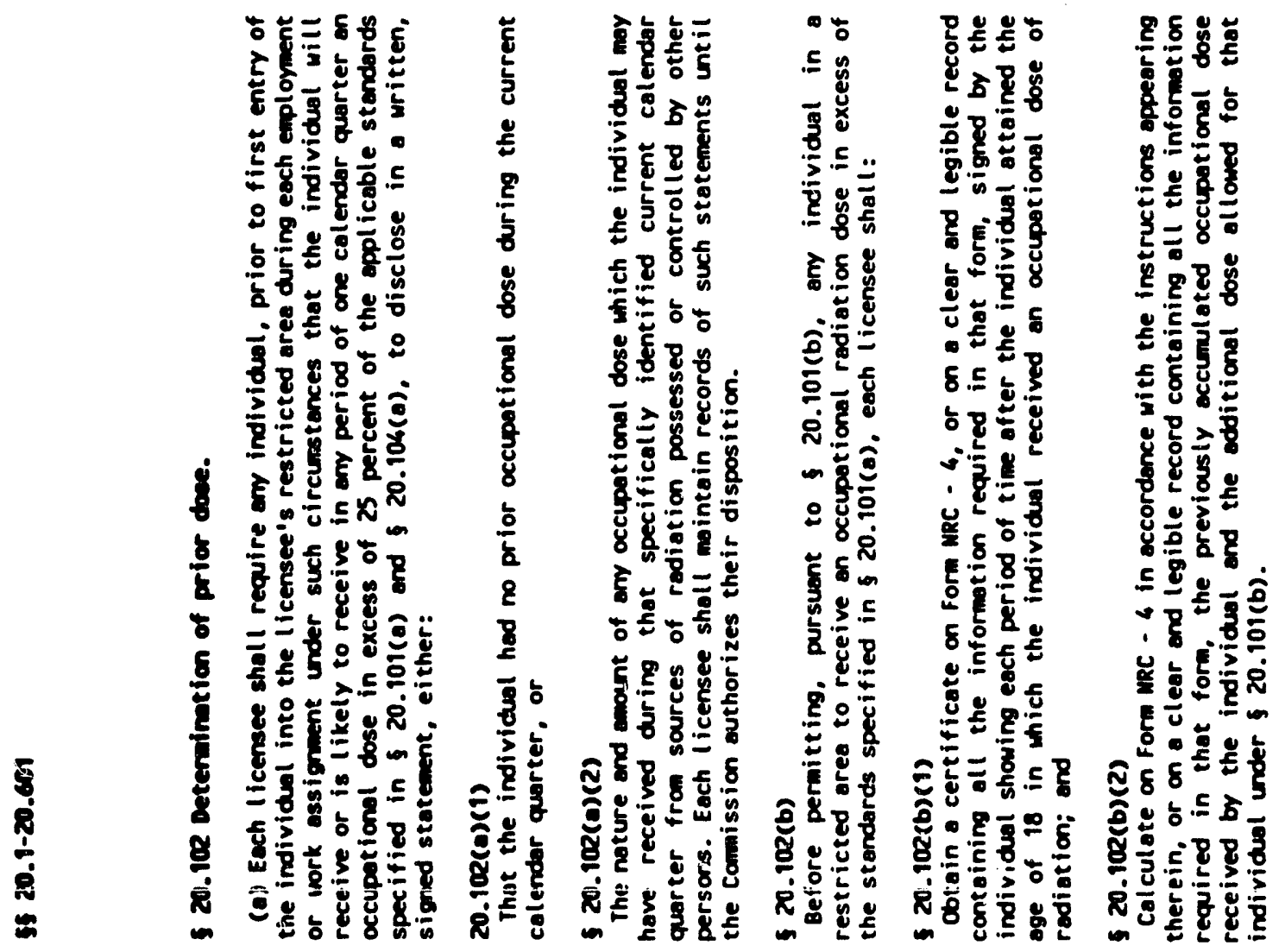



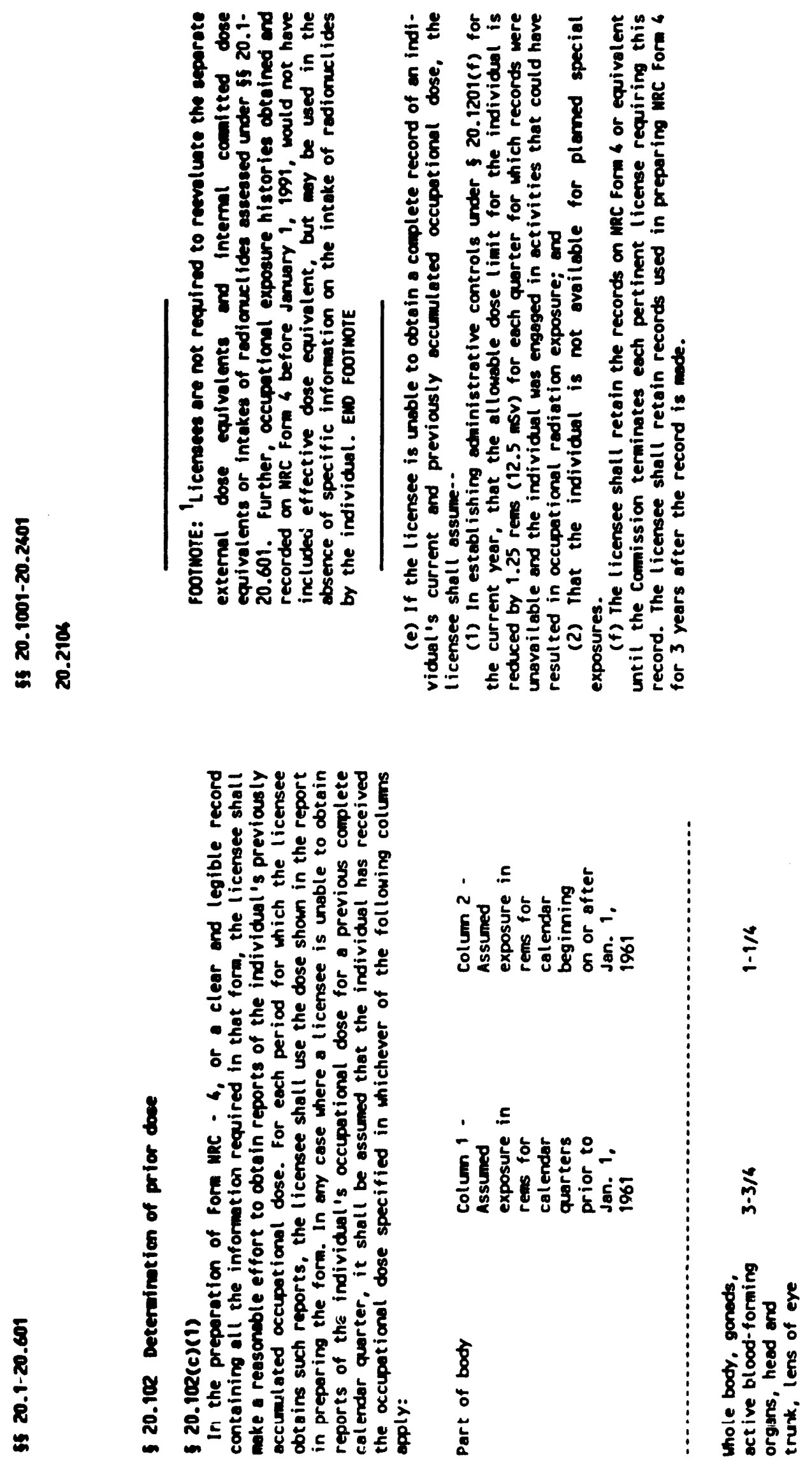

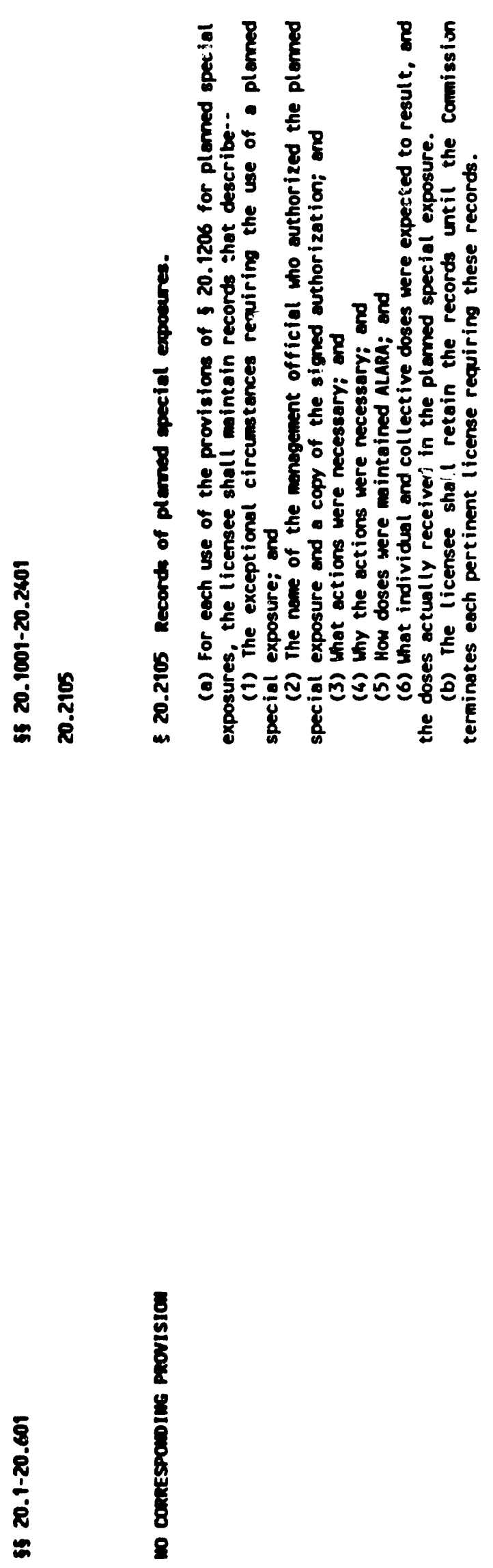


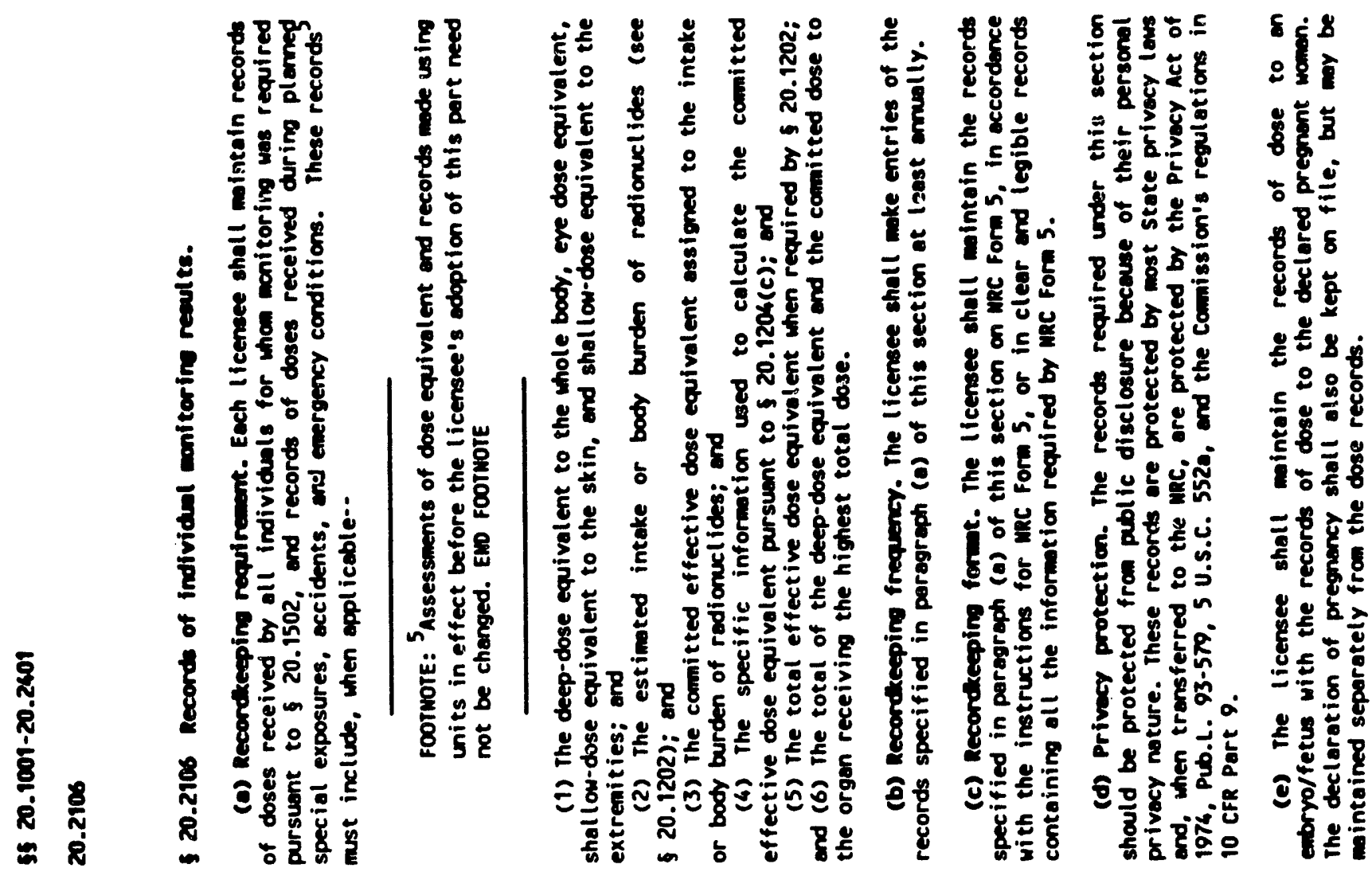

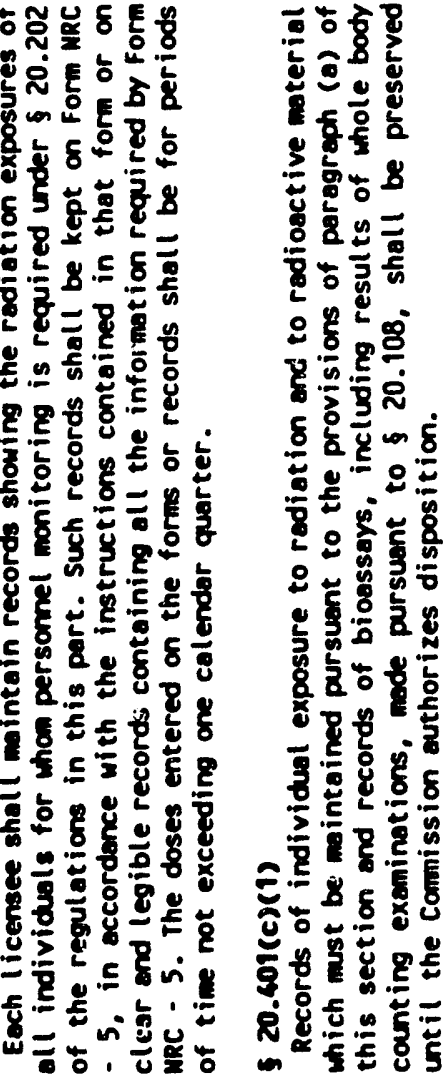




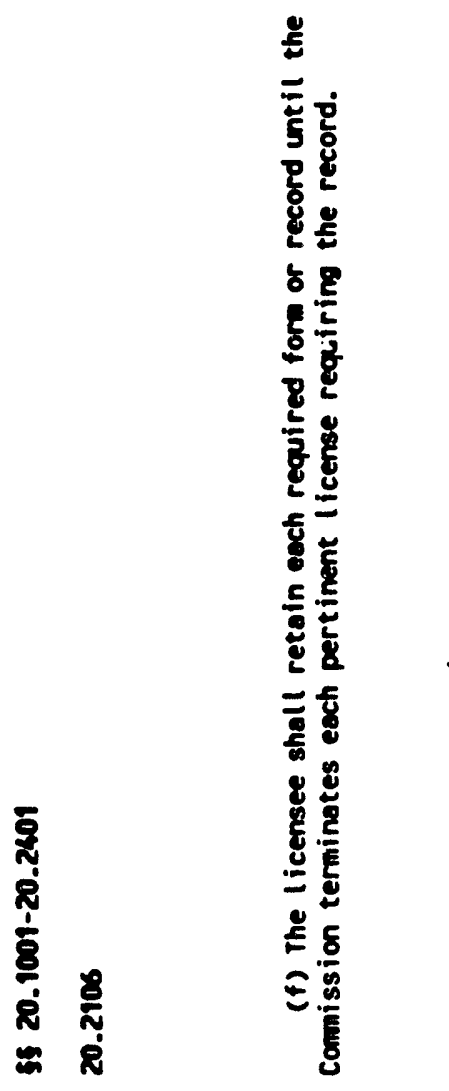

\section{5}

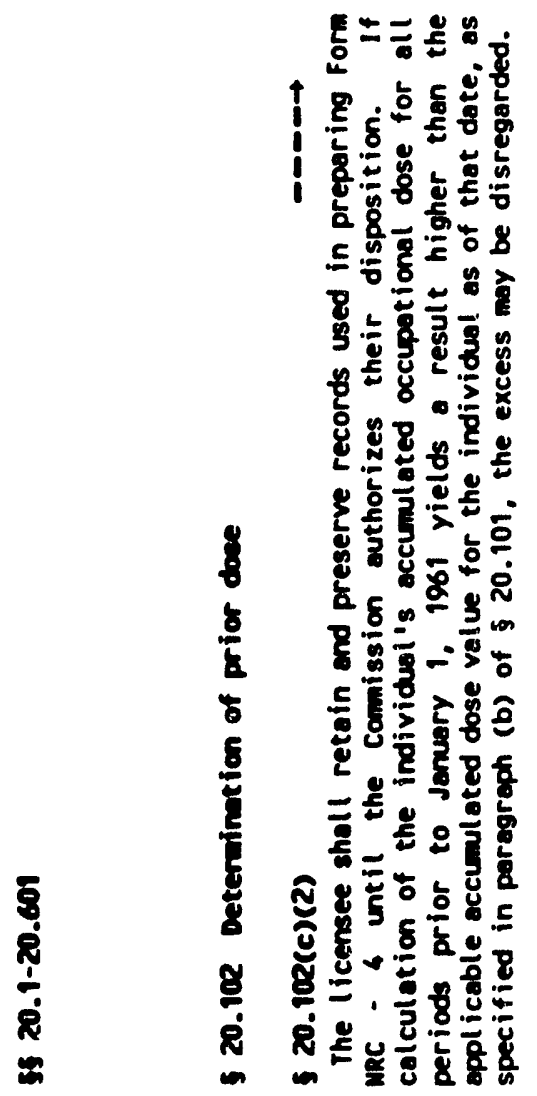



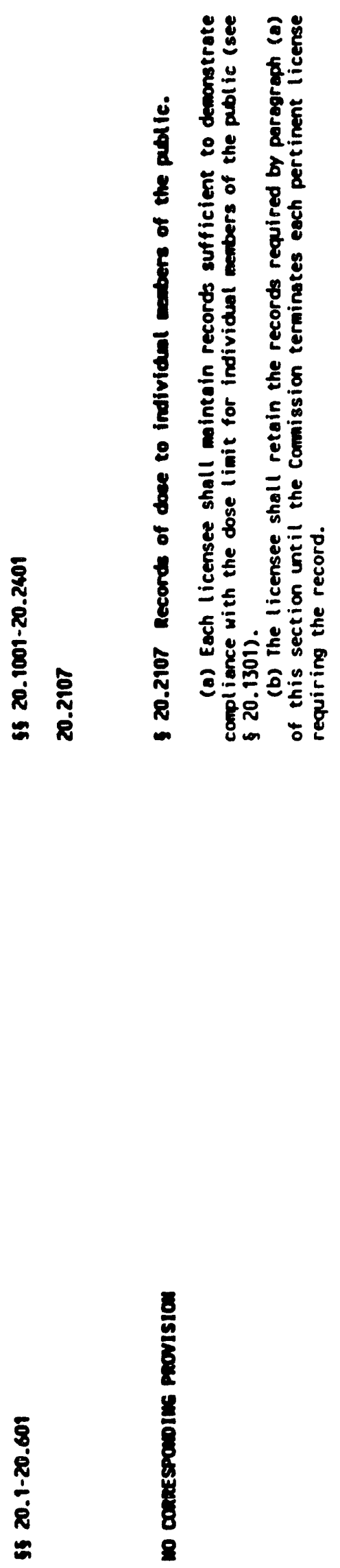


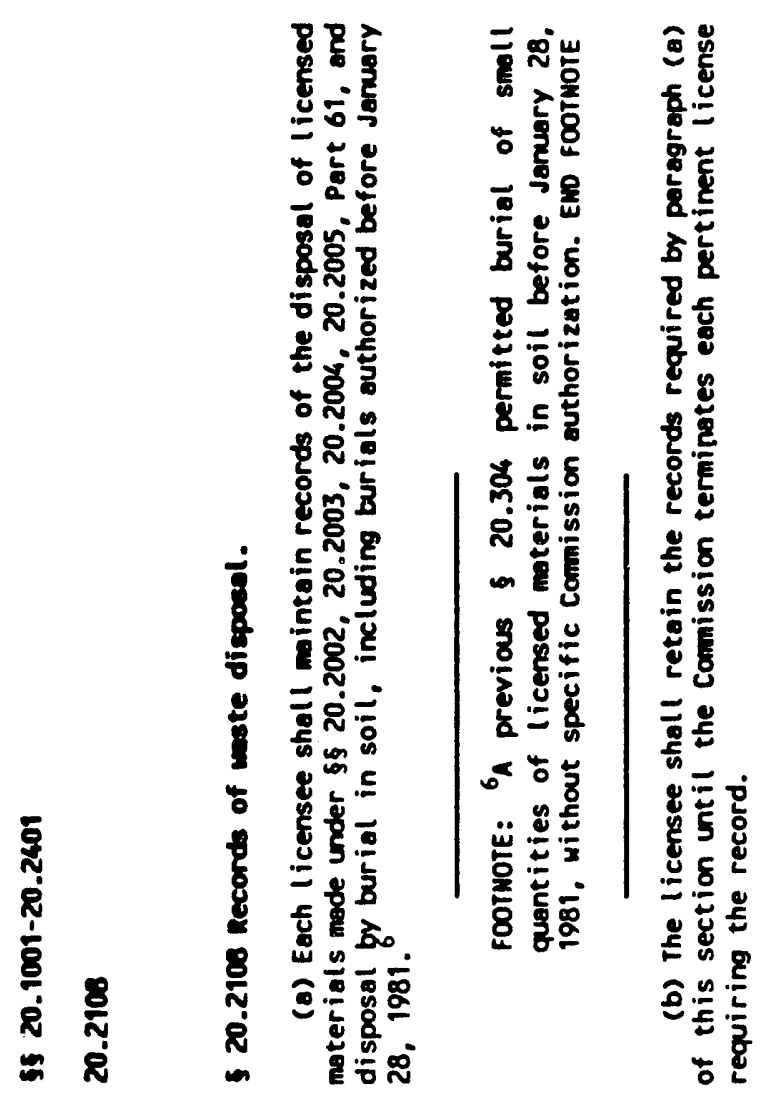

8

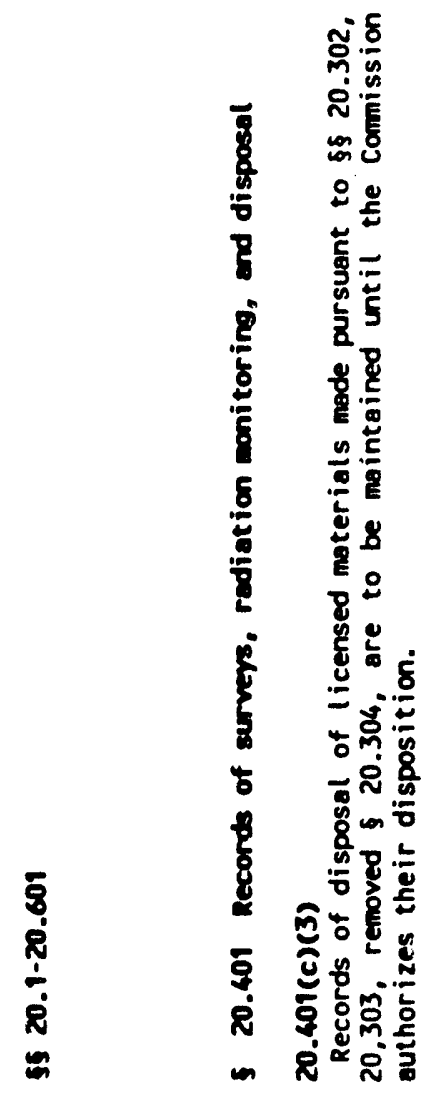



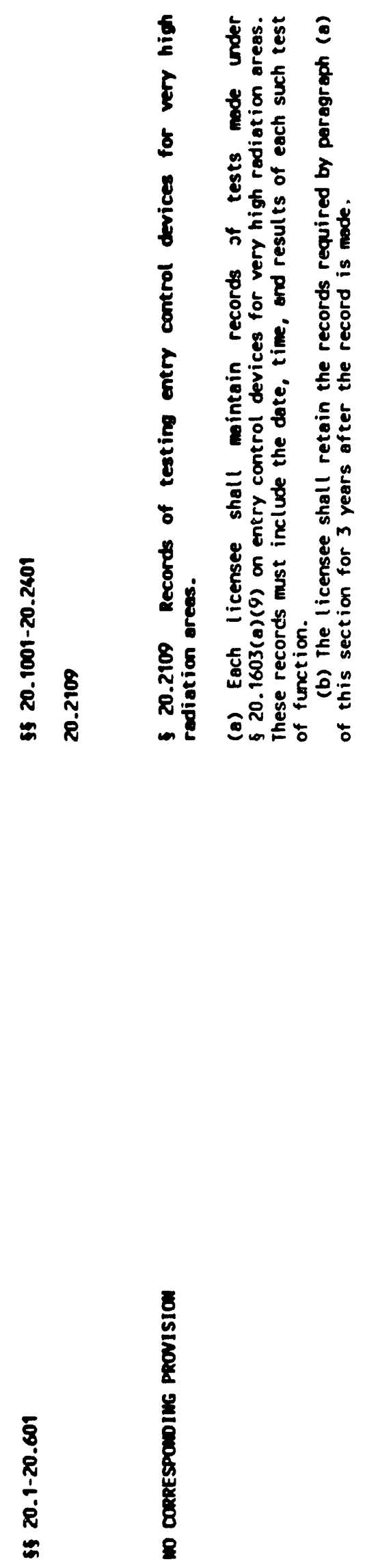

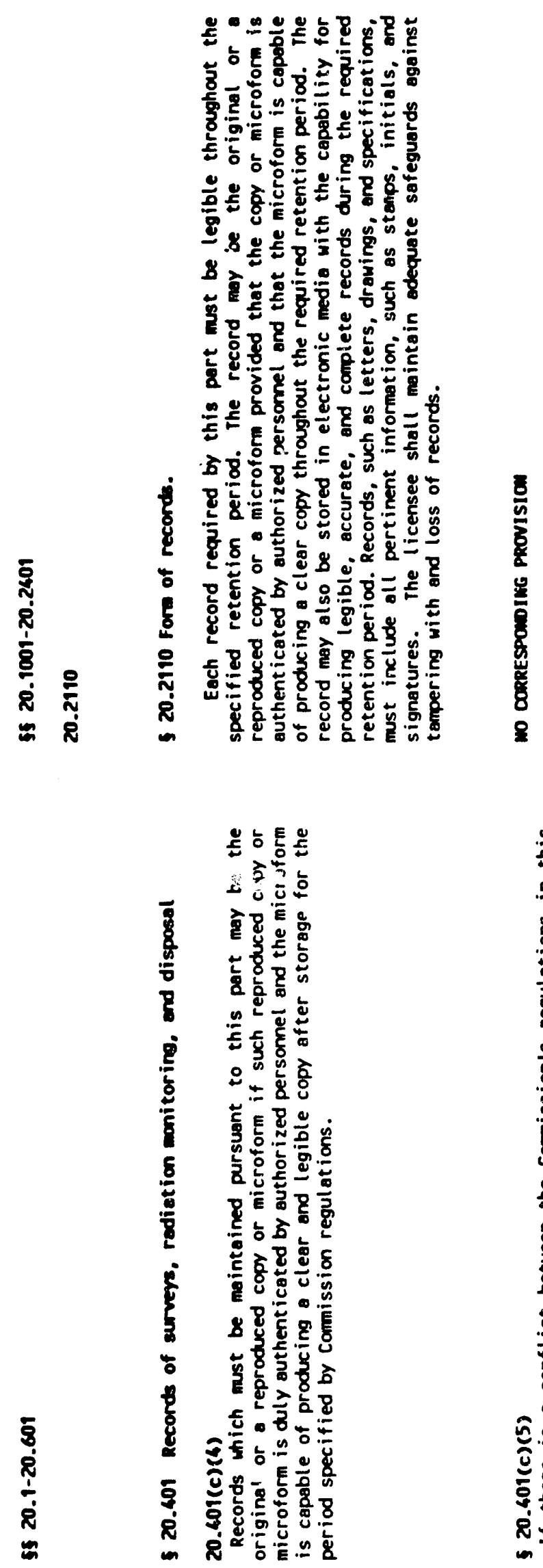

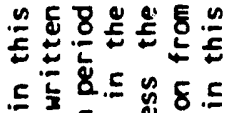

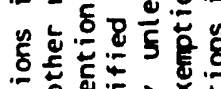

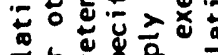

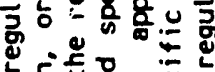

我安 $8=$

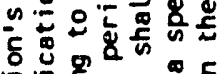

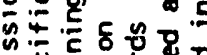

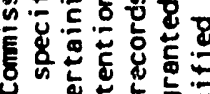

西过的

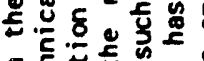

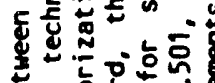

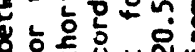

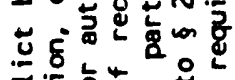

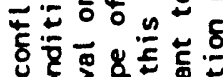

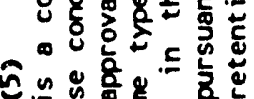

仓。气

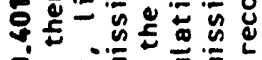

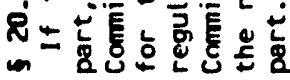




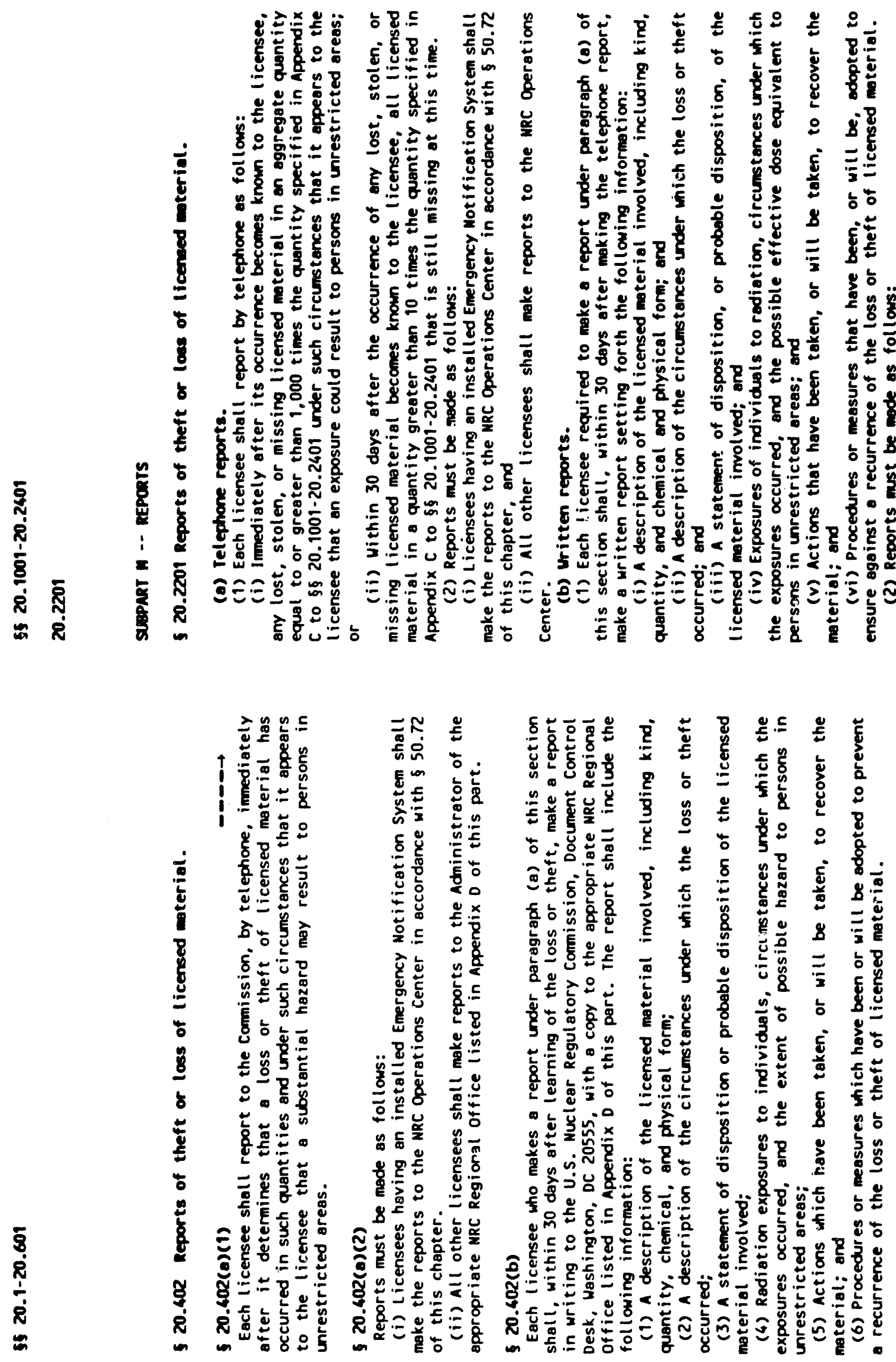



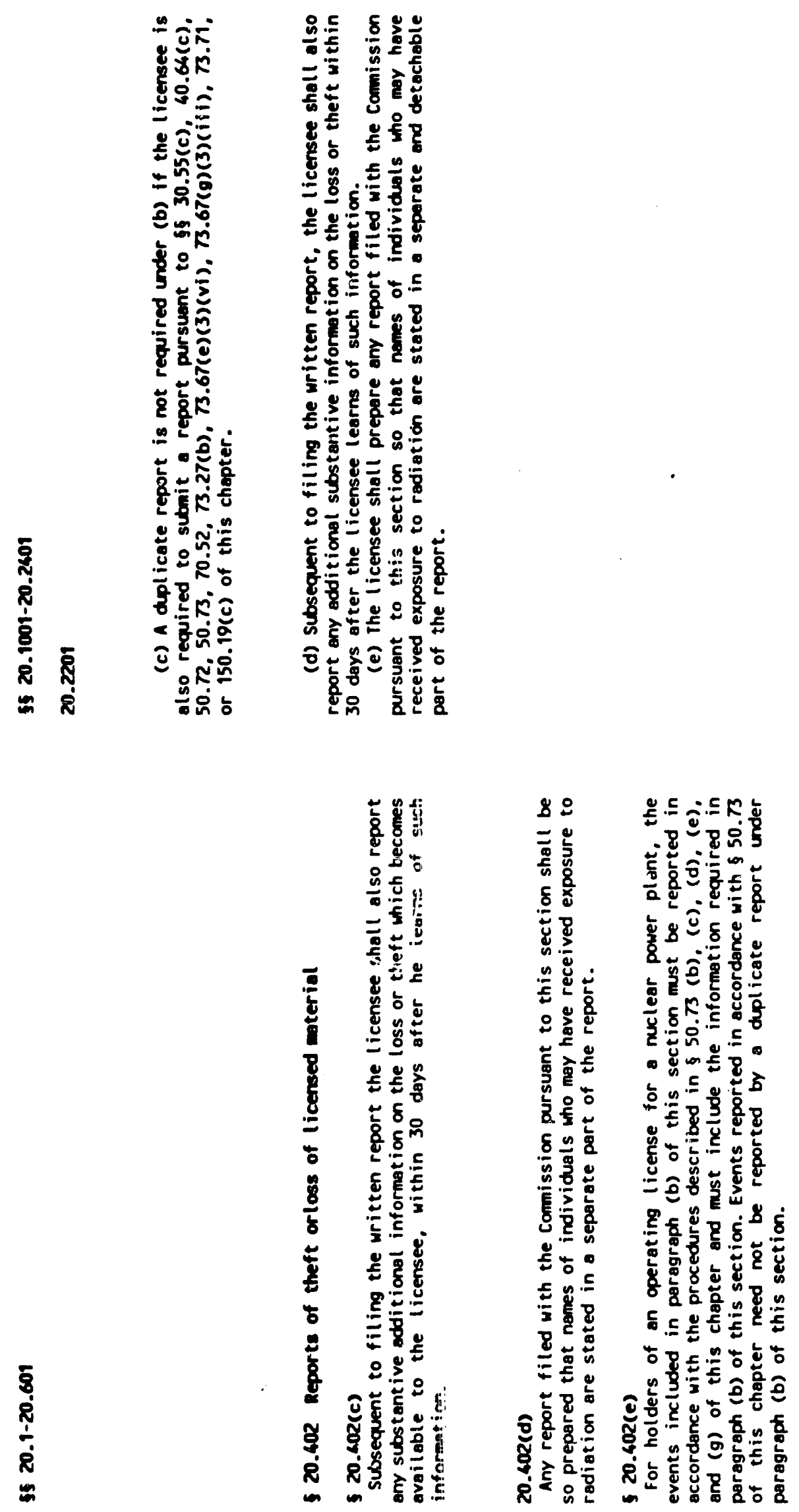

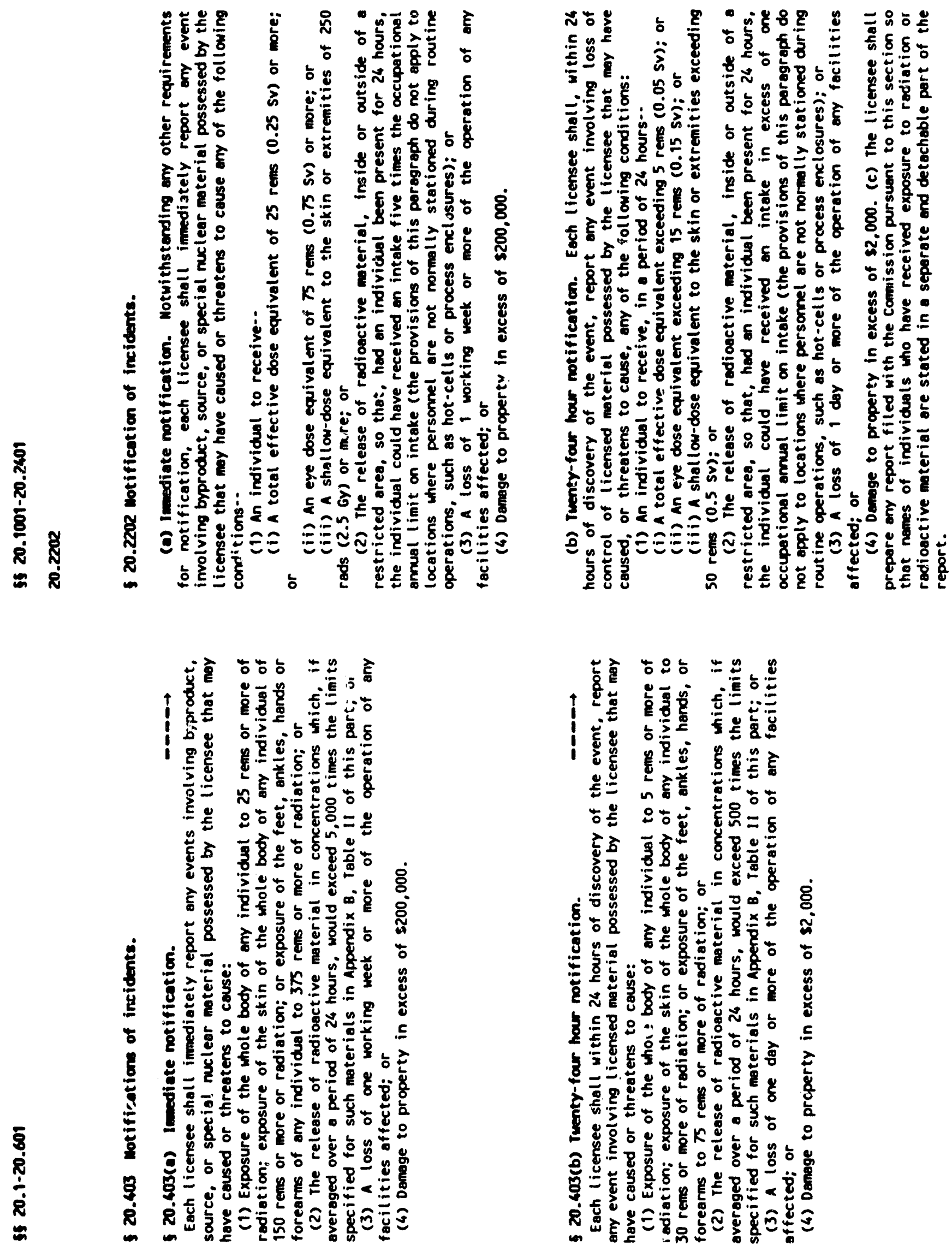

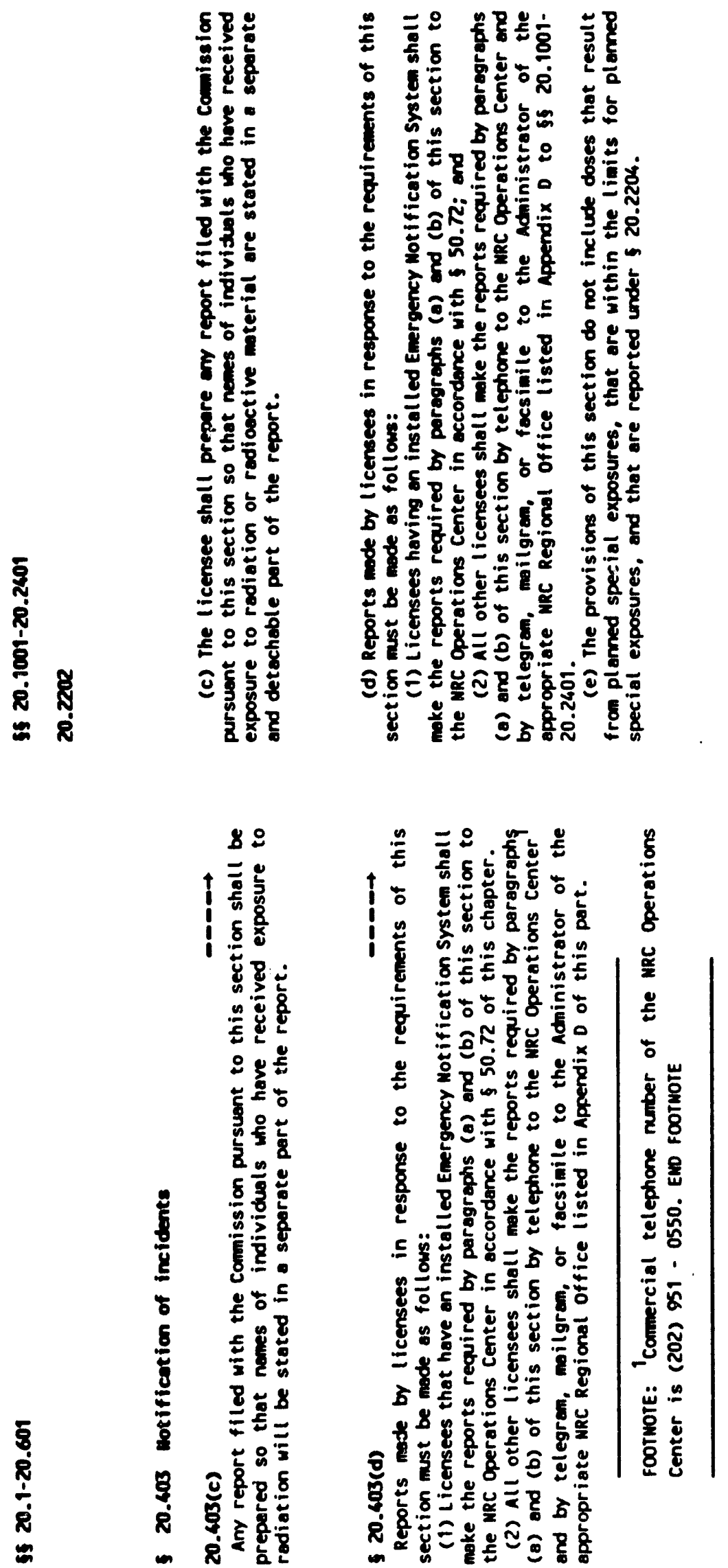


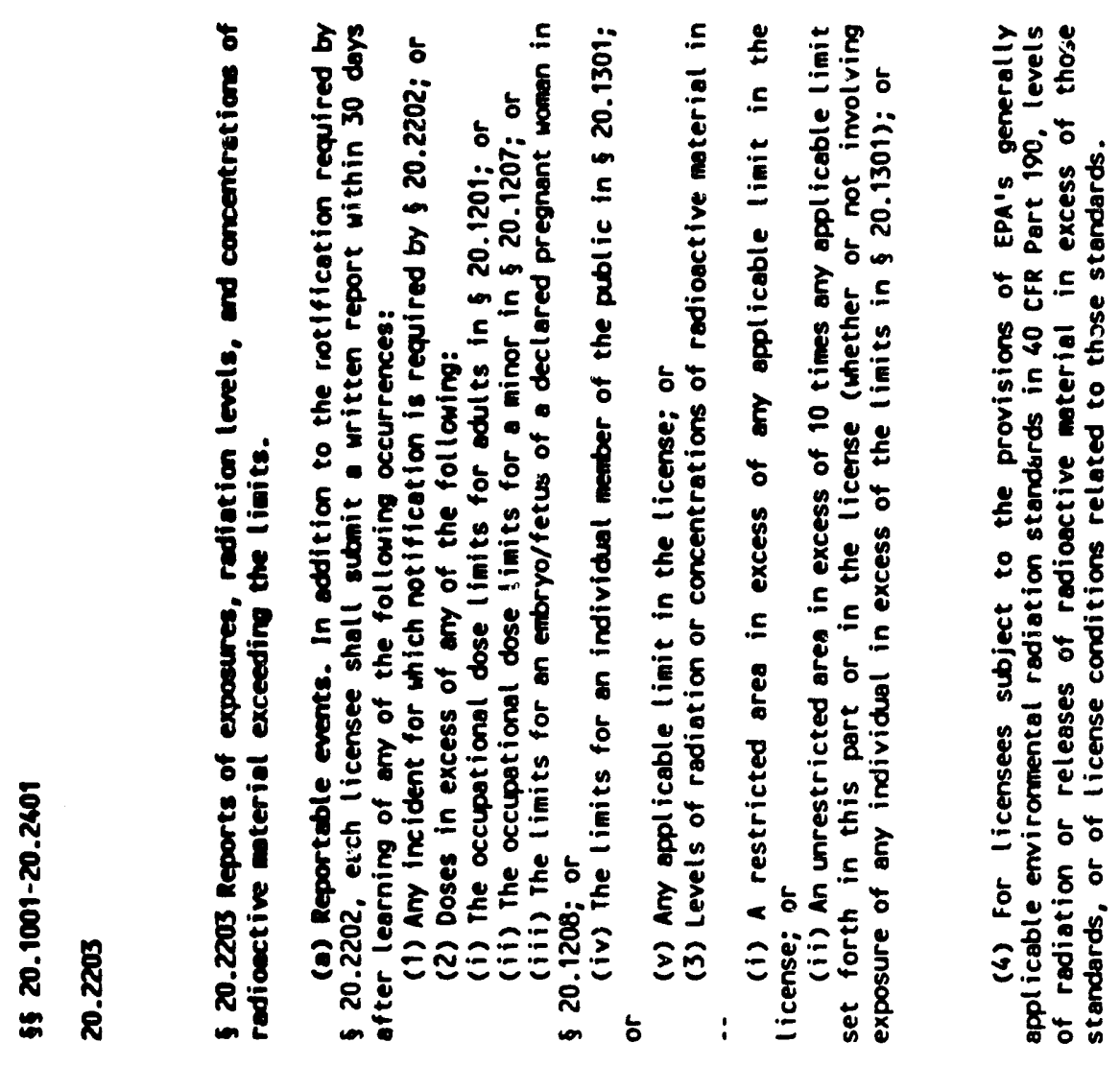

2
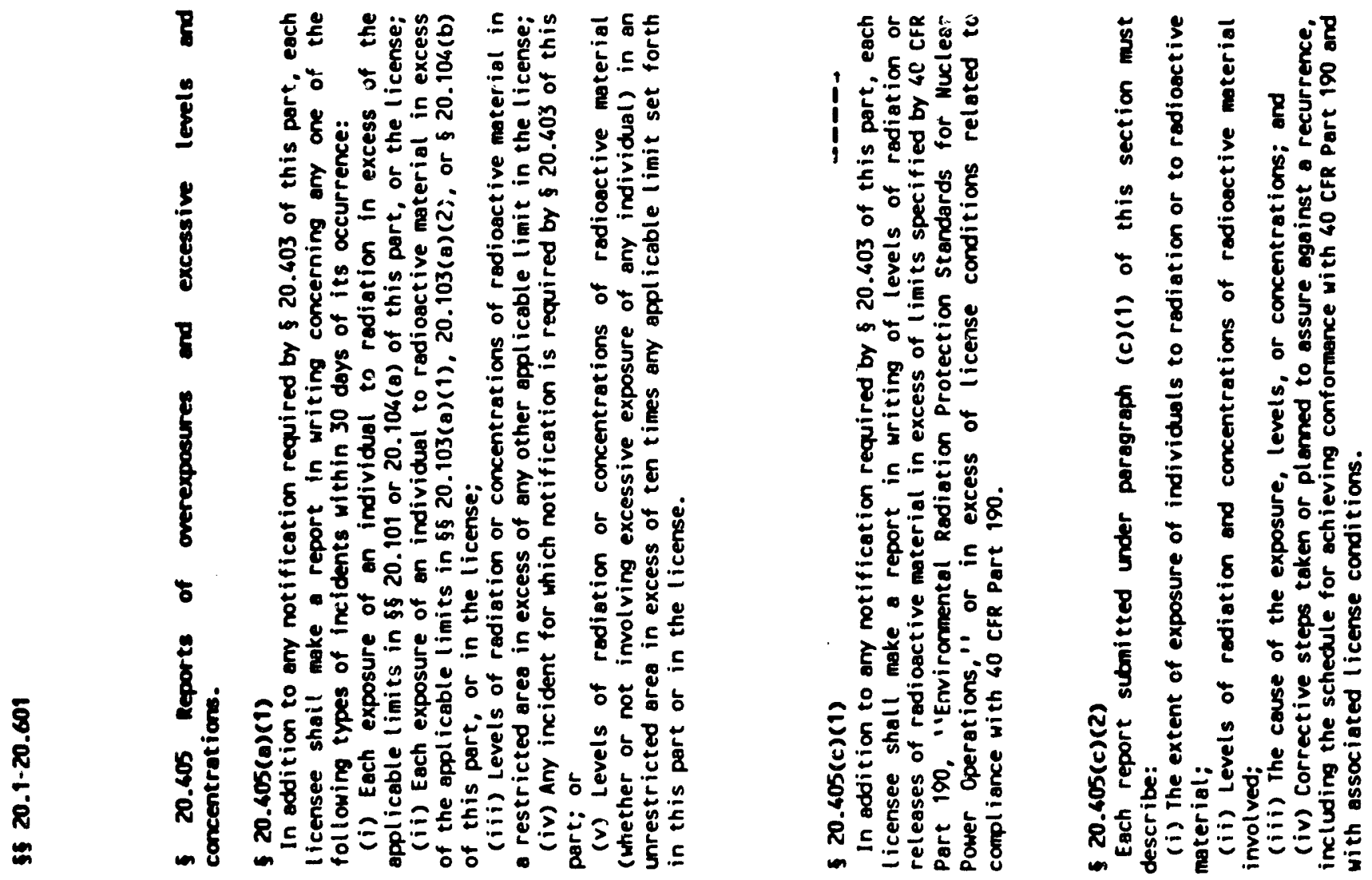

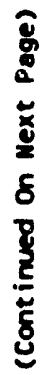




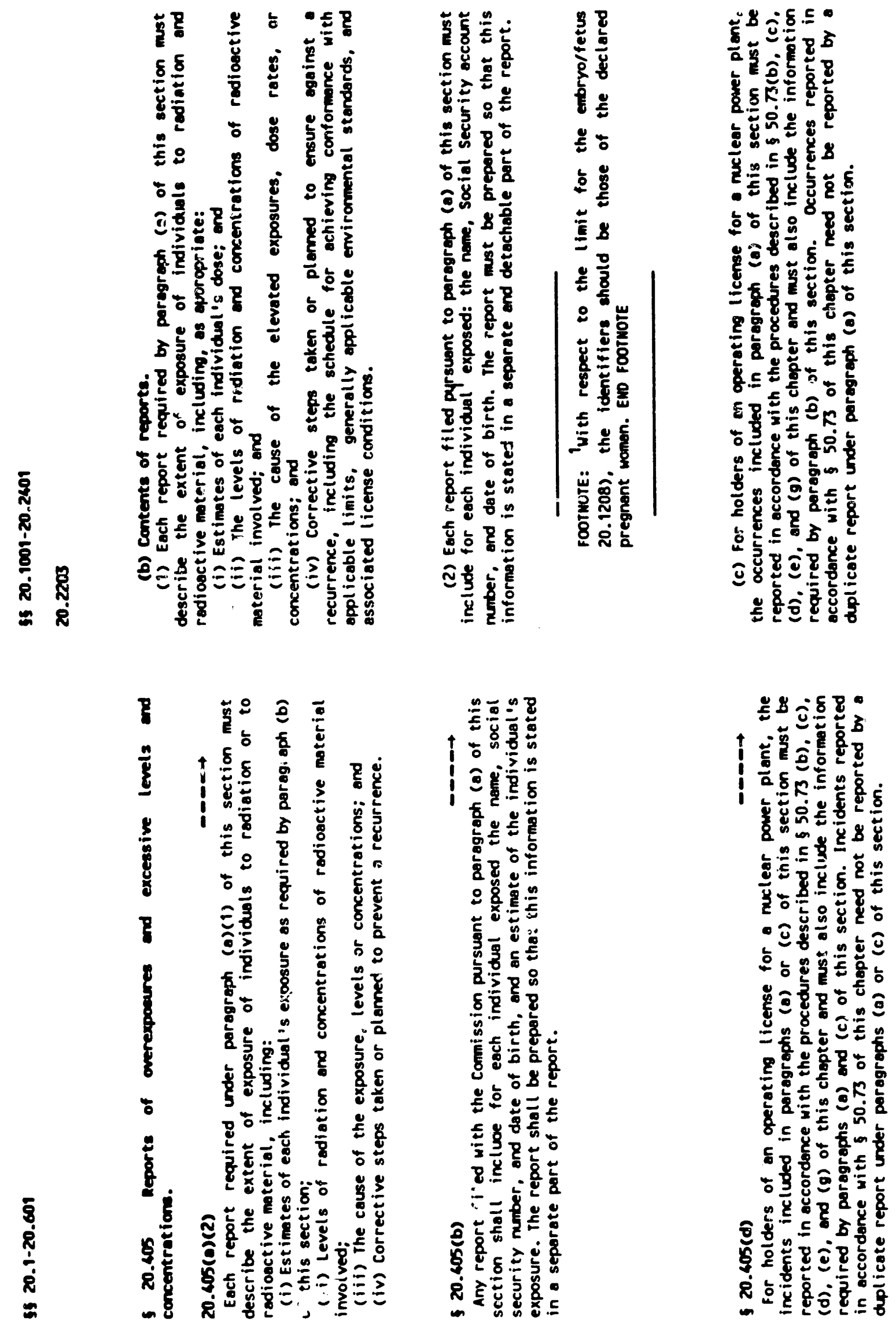



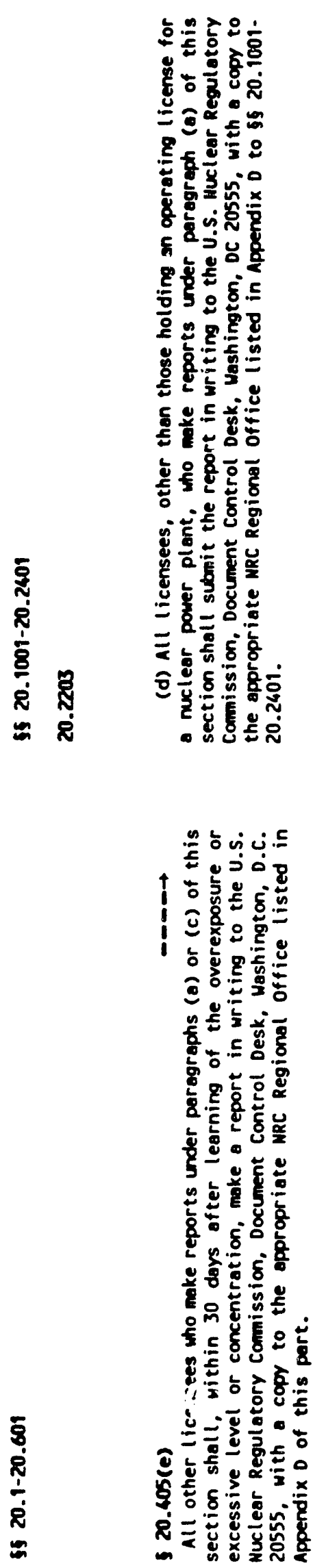

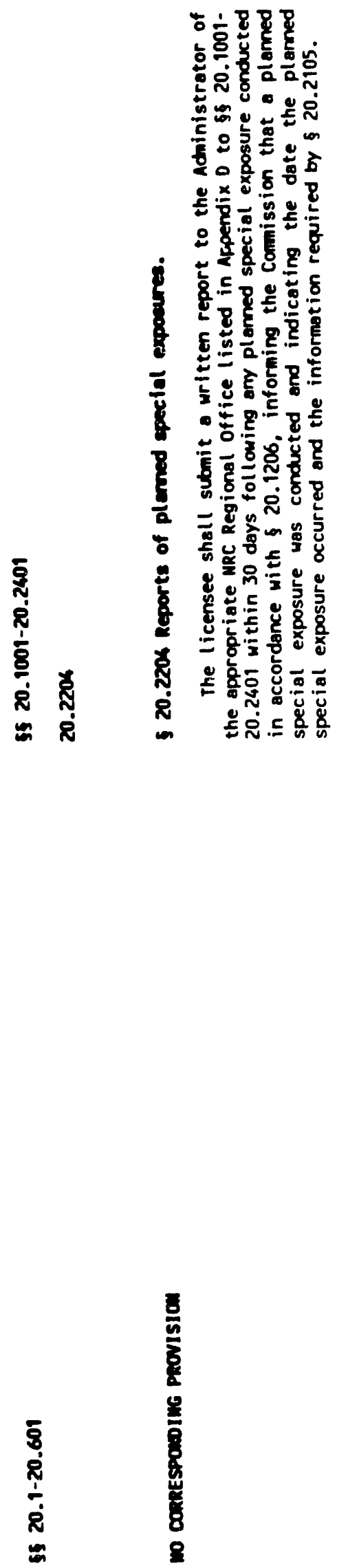


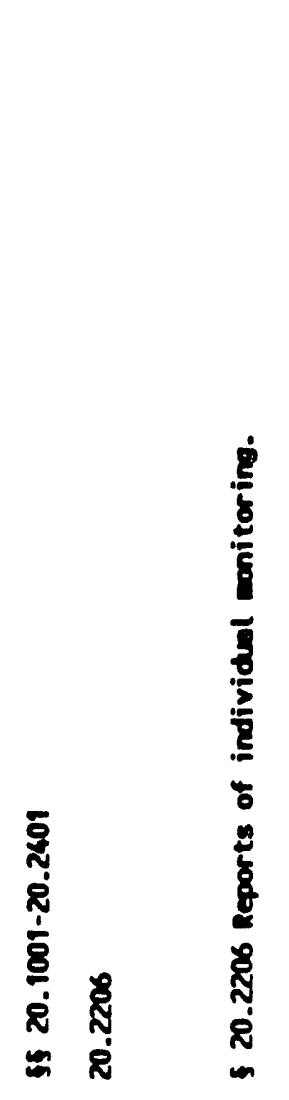

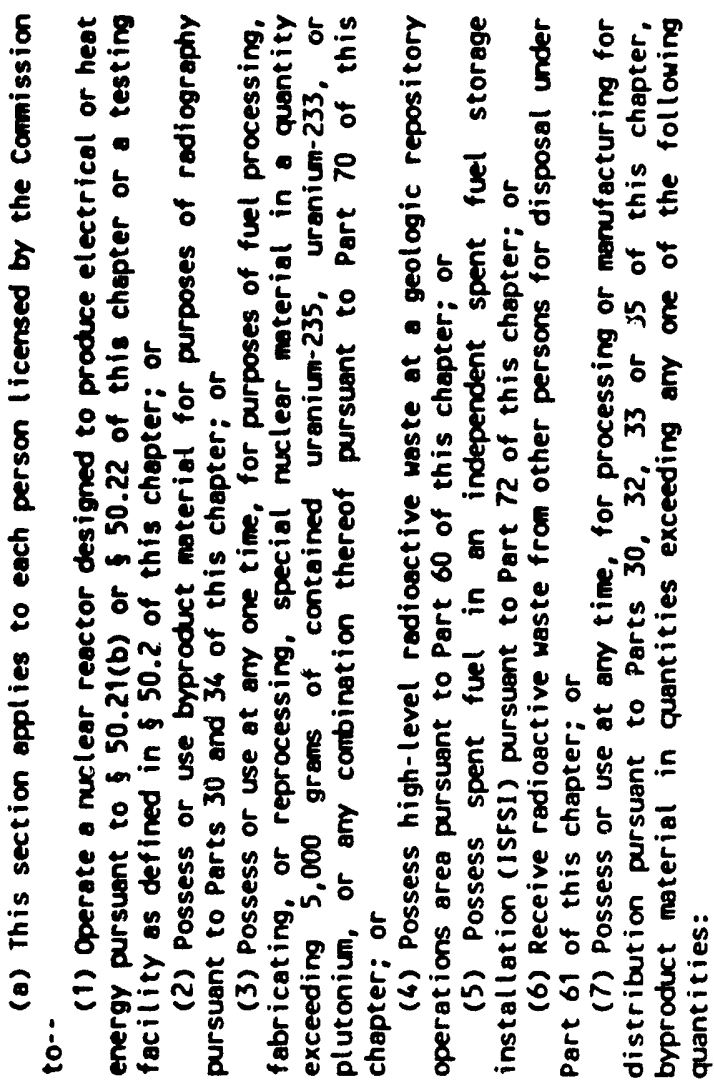

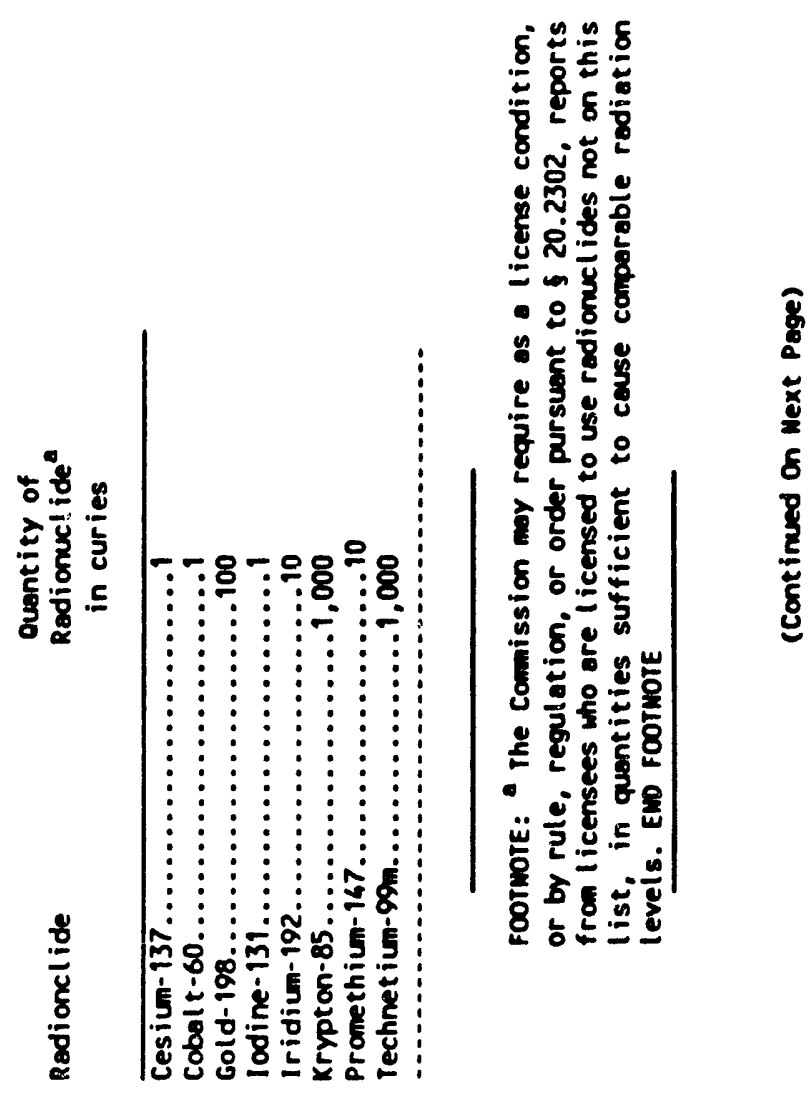

8

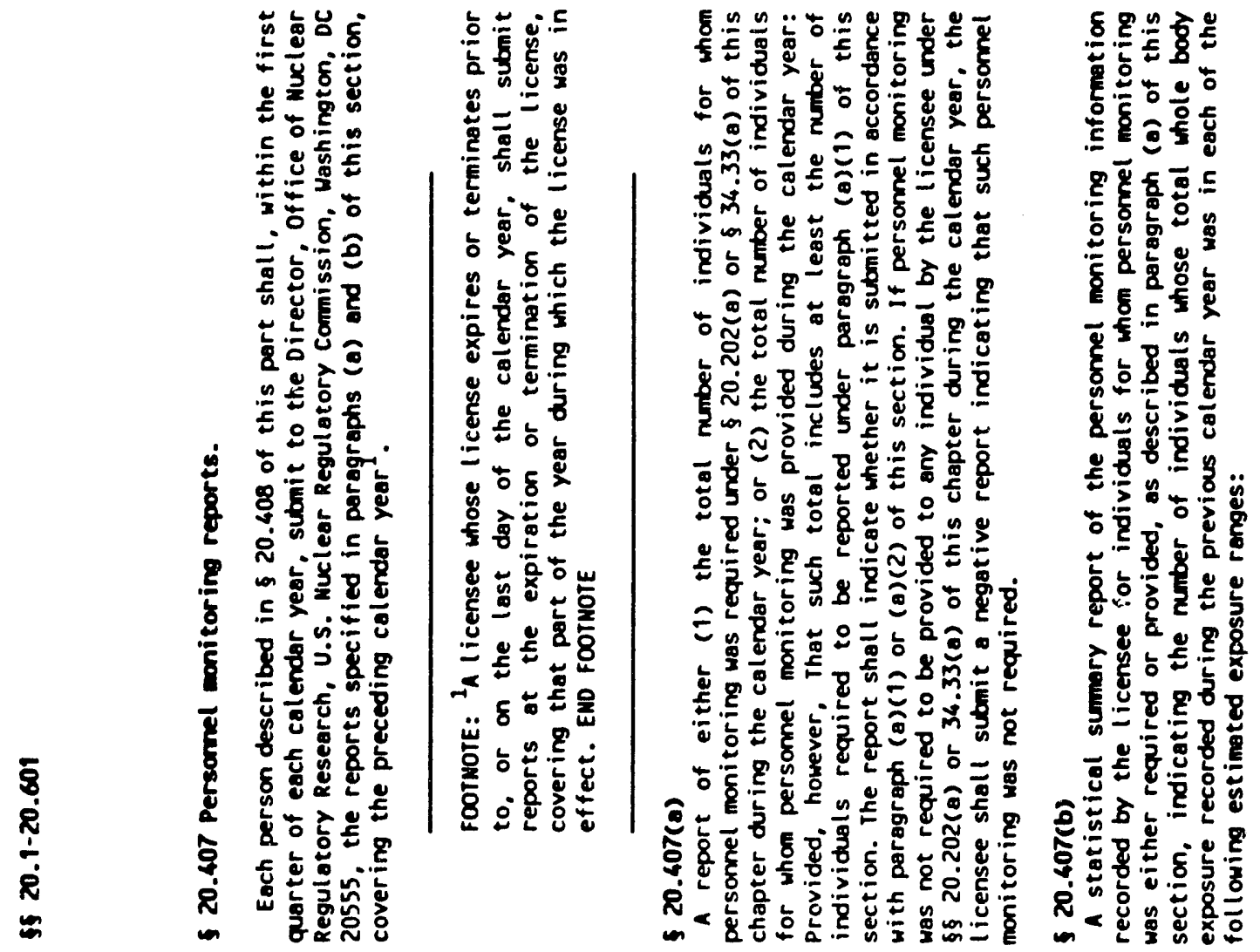



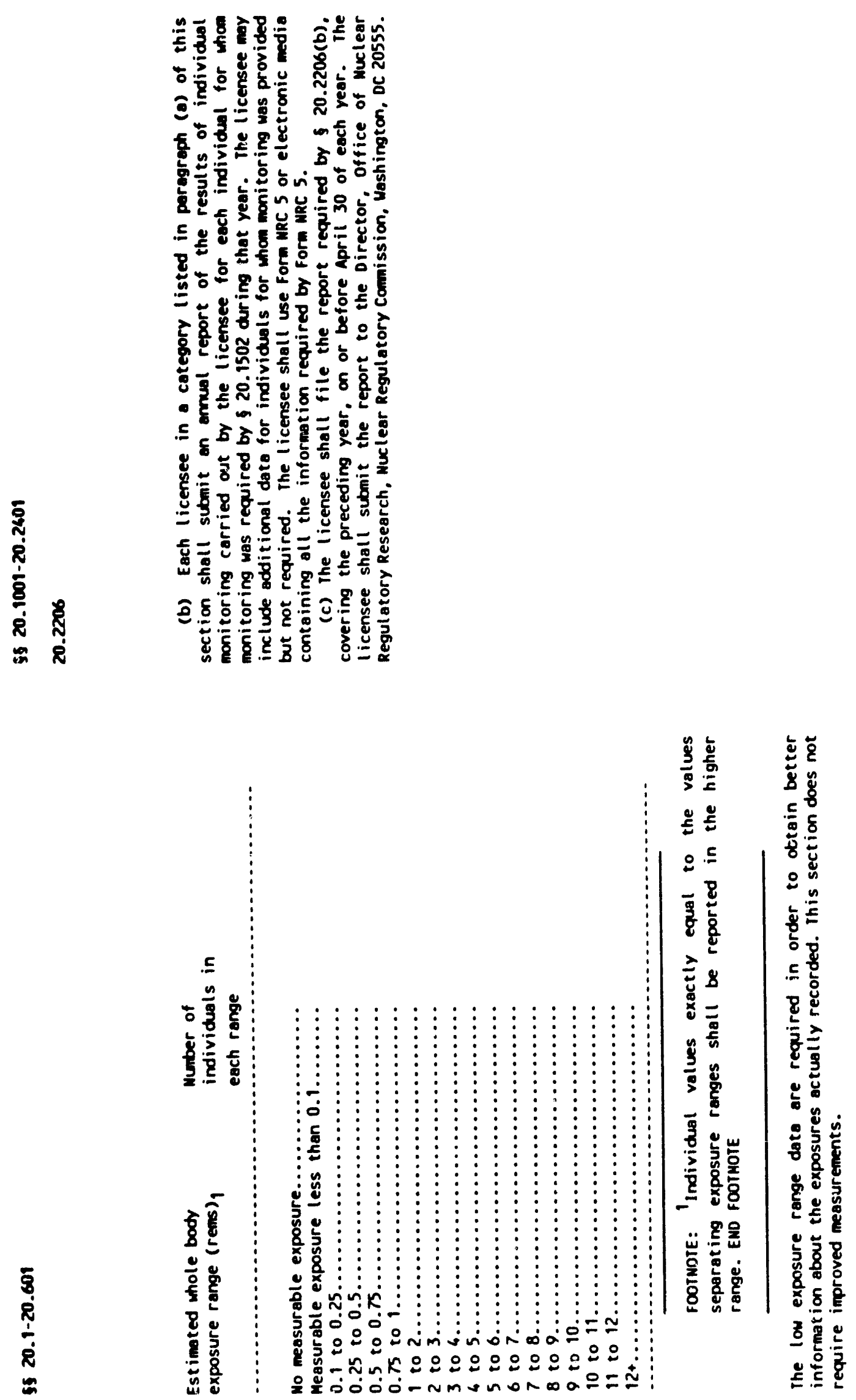

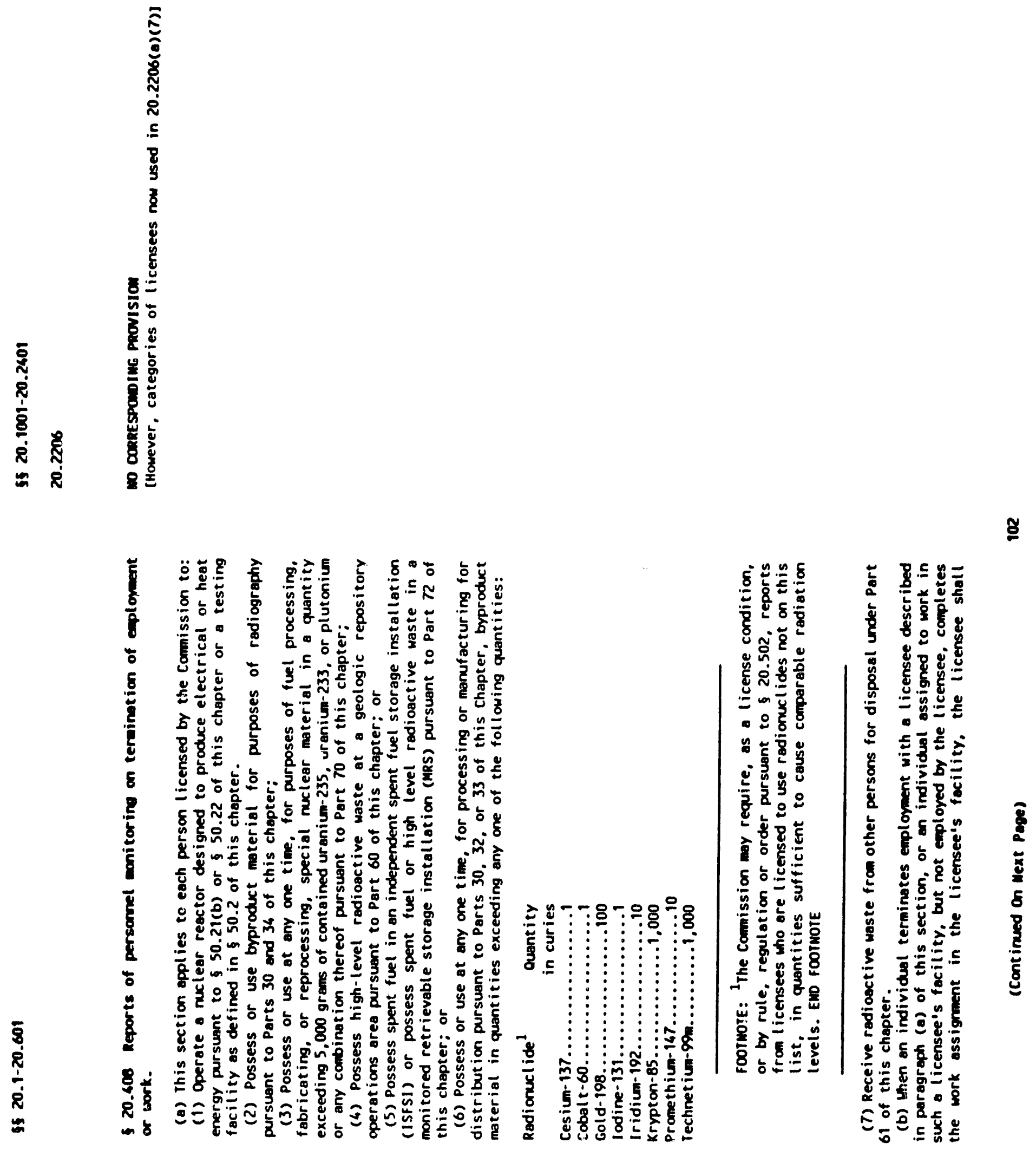

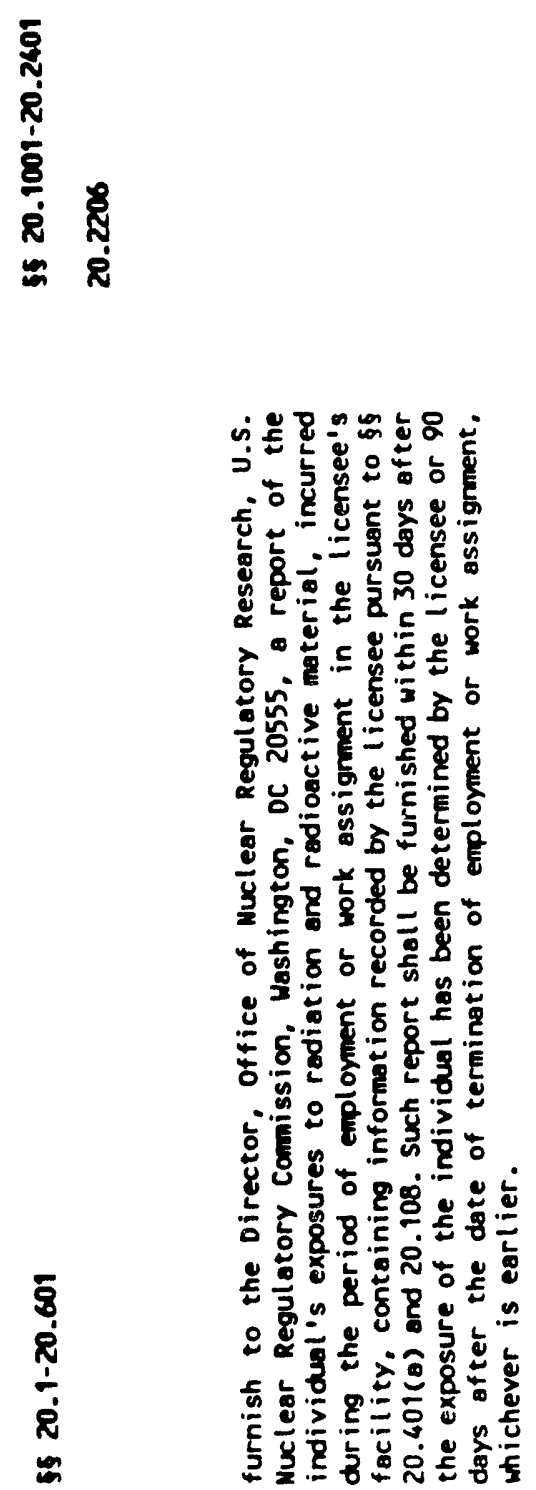

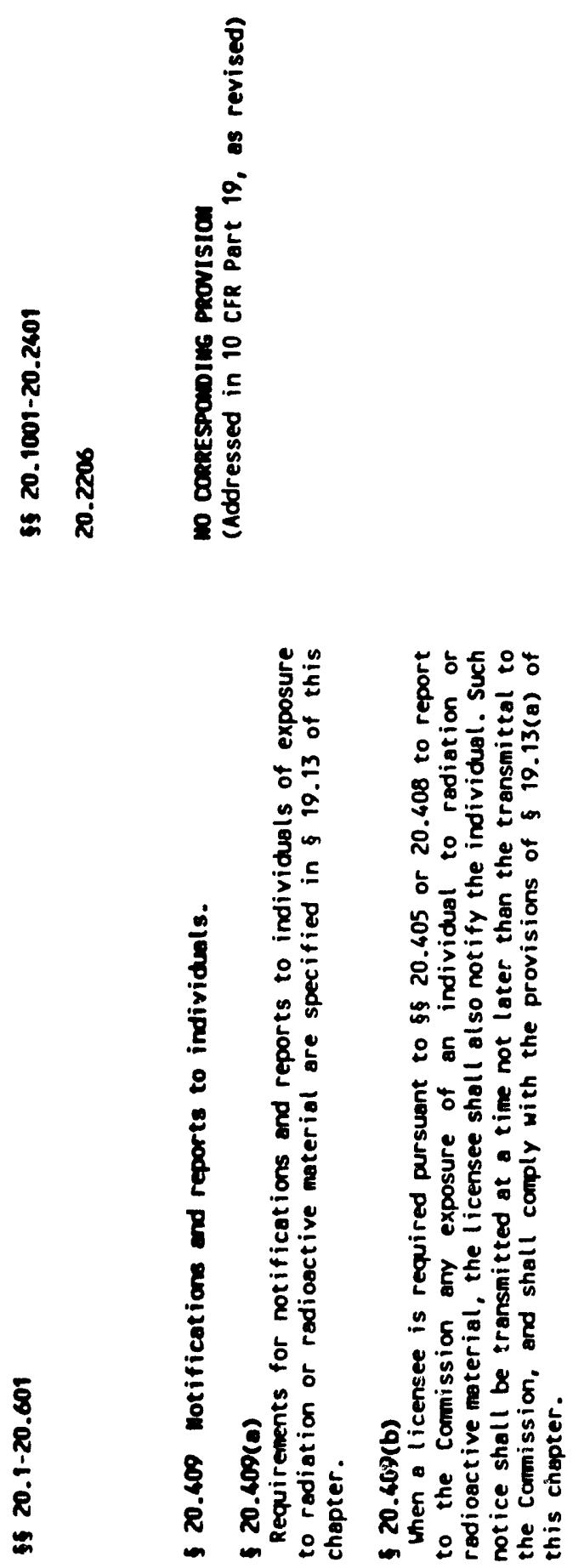

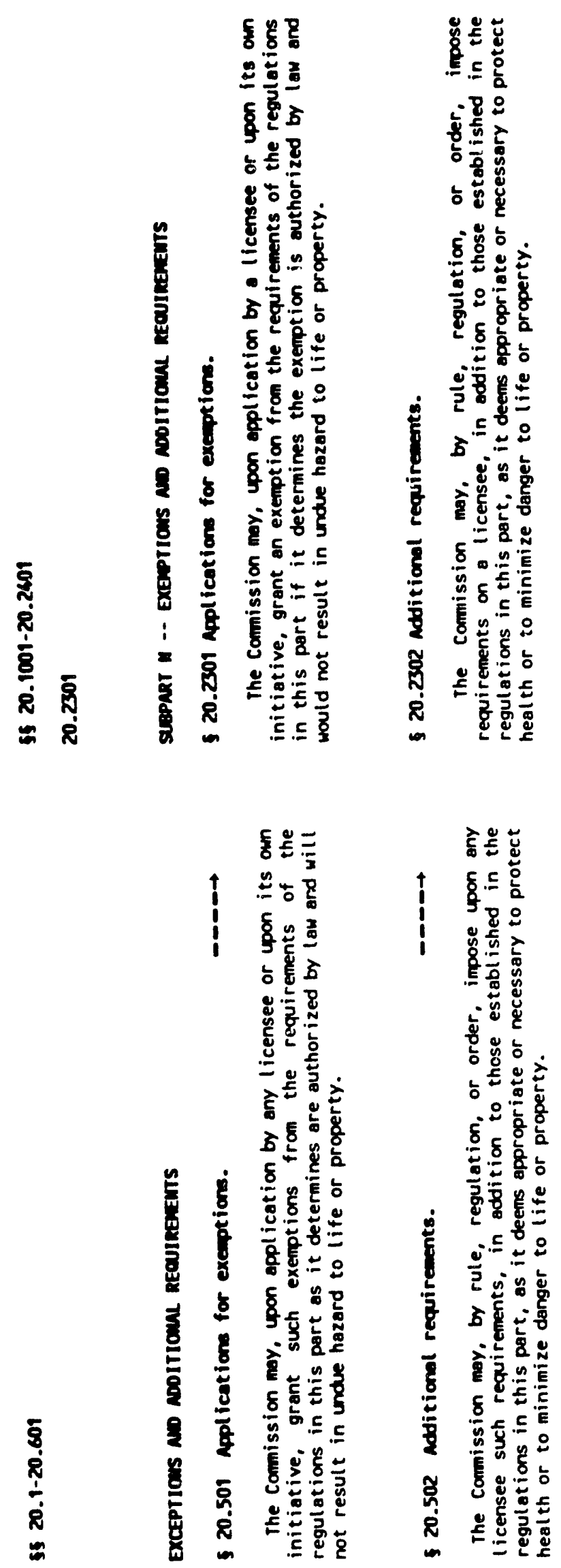

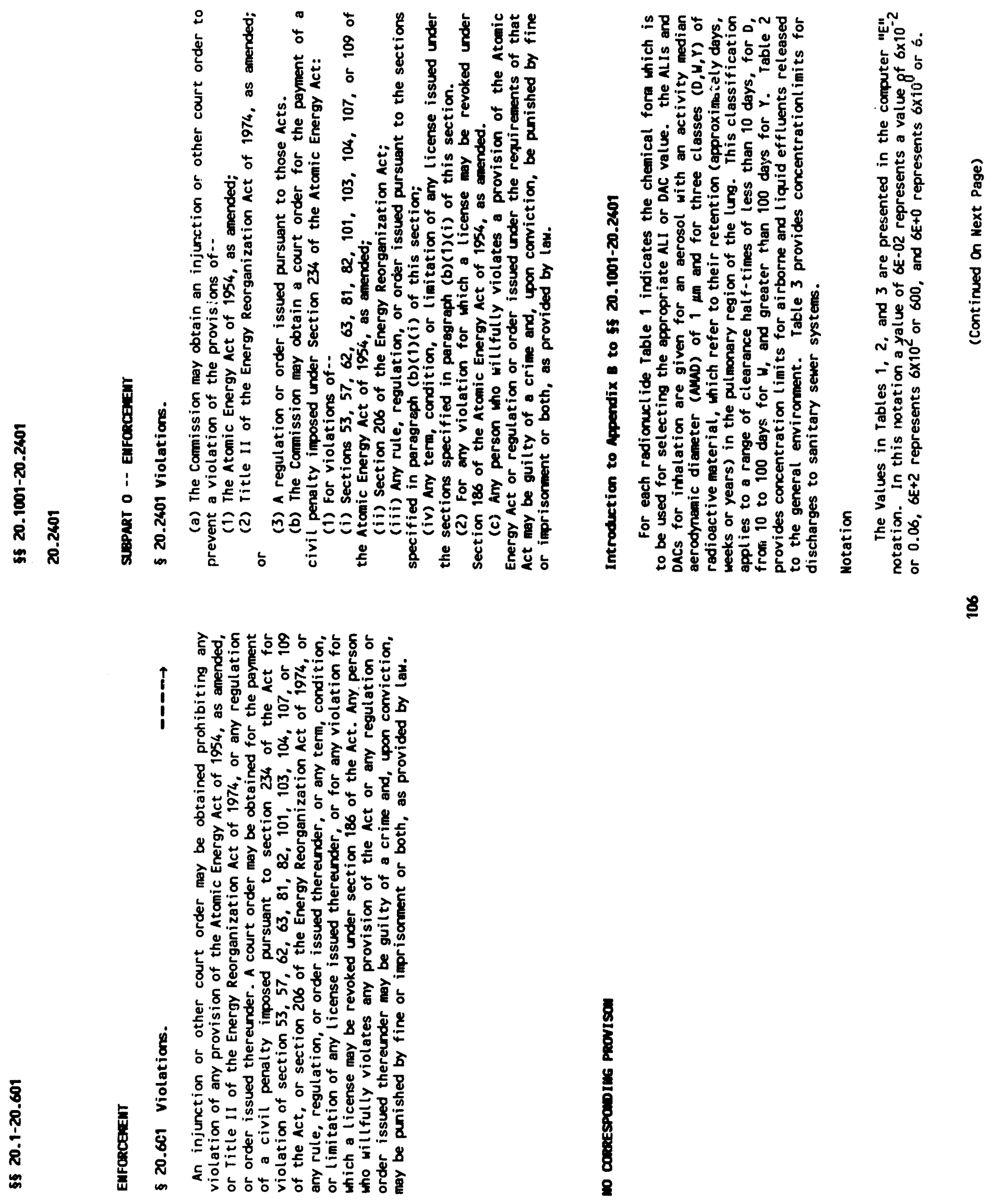

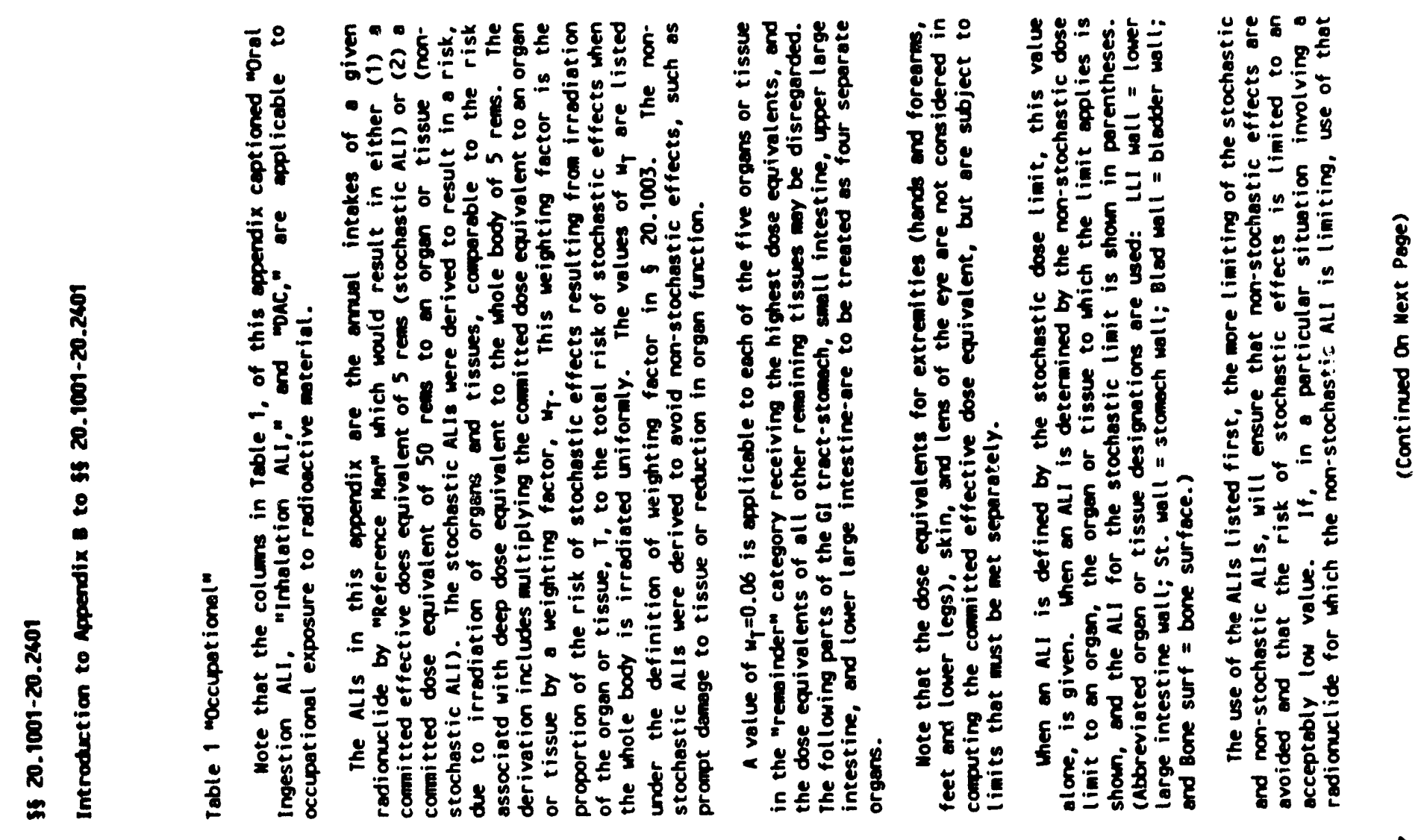

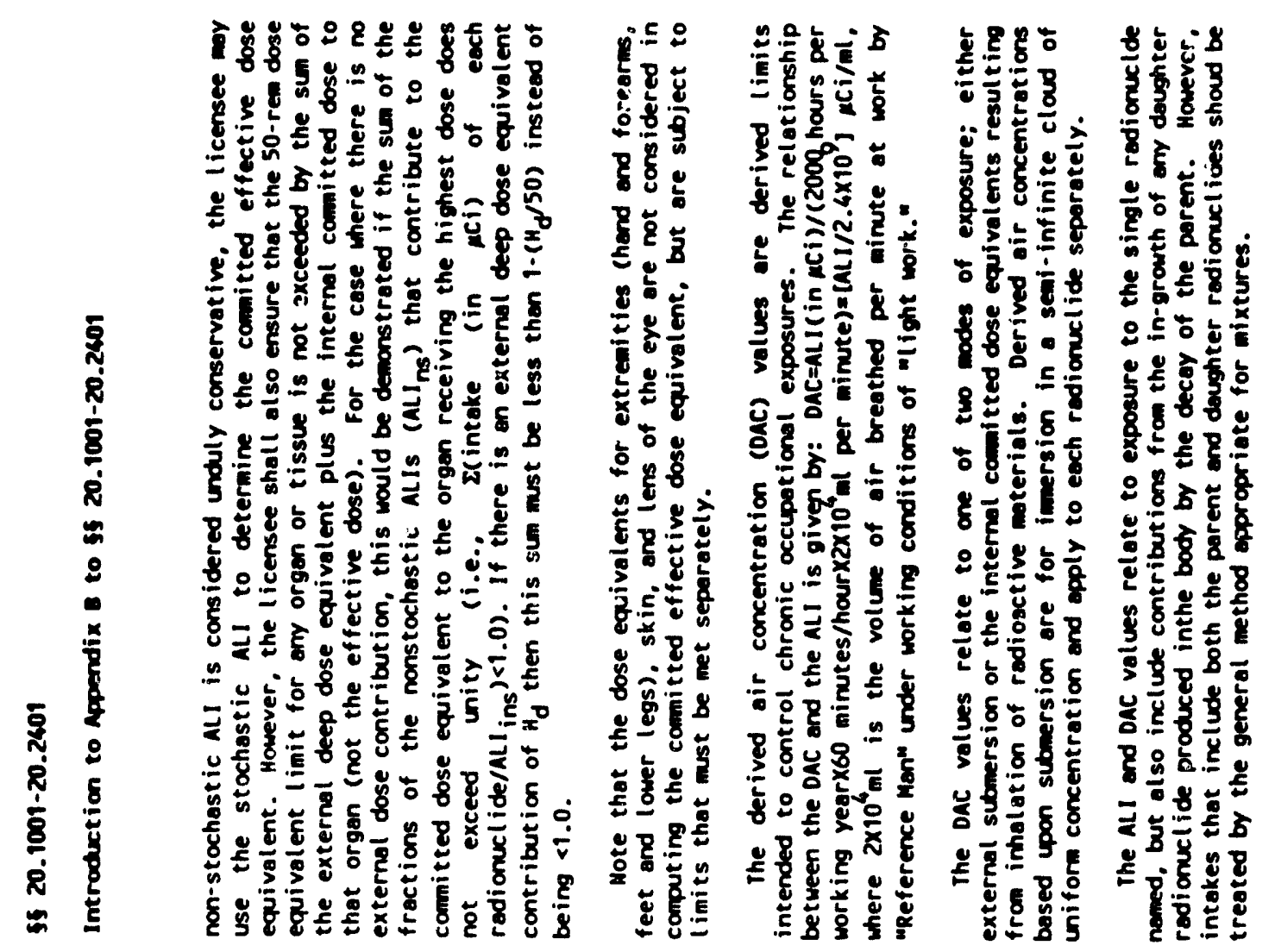

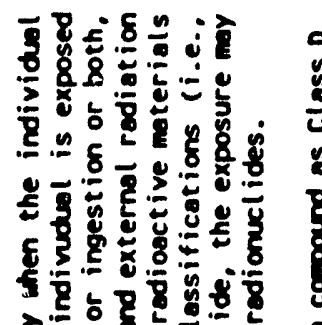

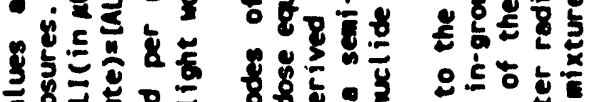

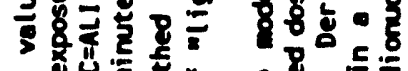

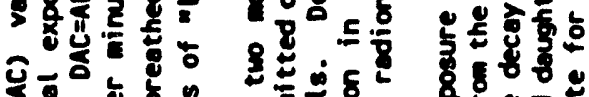

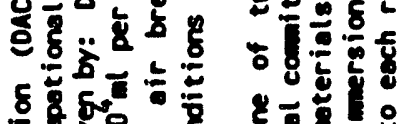

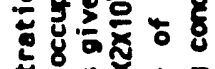

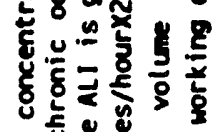

ชै है

ชั

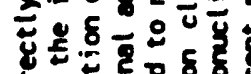

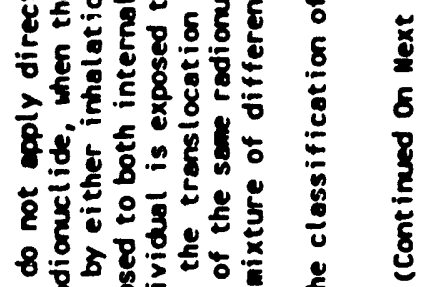

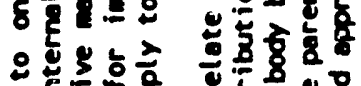

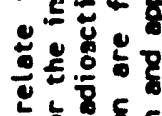

-

58.8

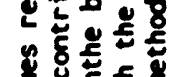

今8 8 \%

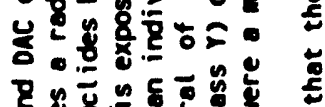

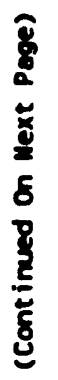

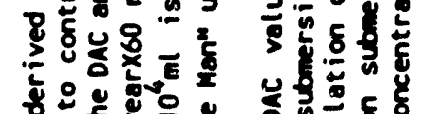

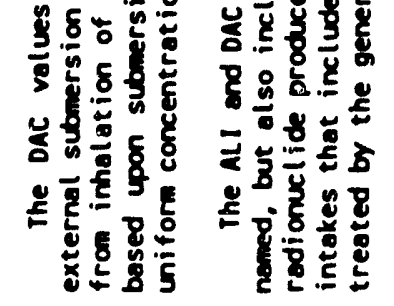

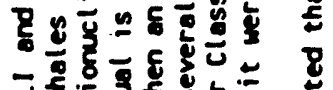

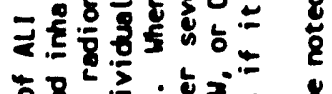

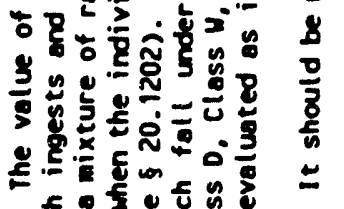

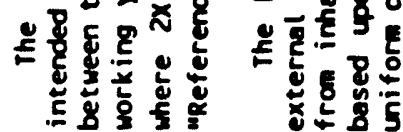

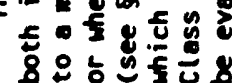



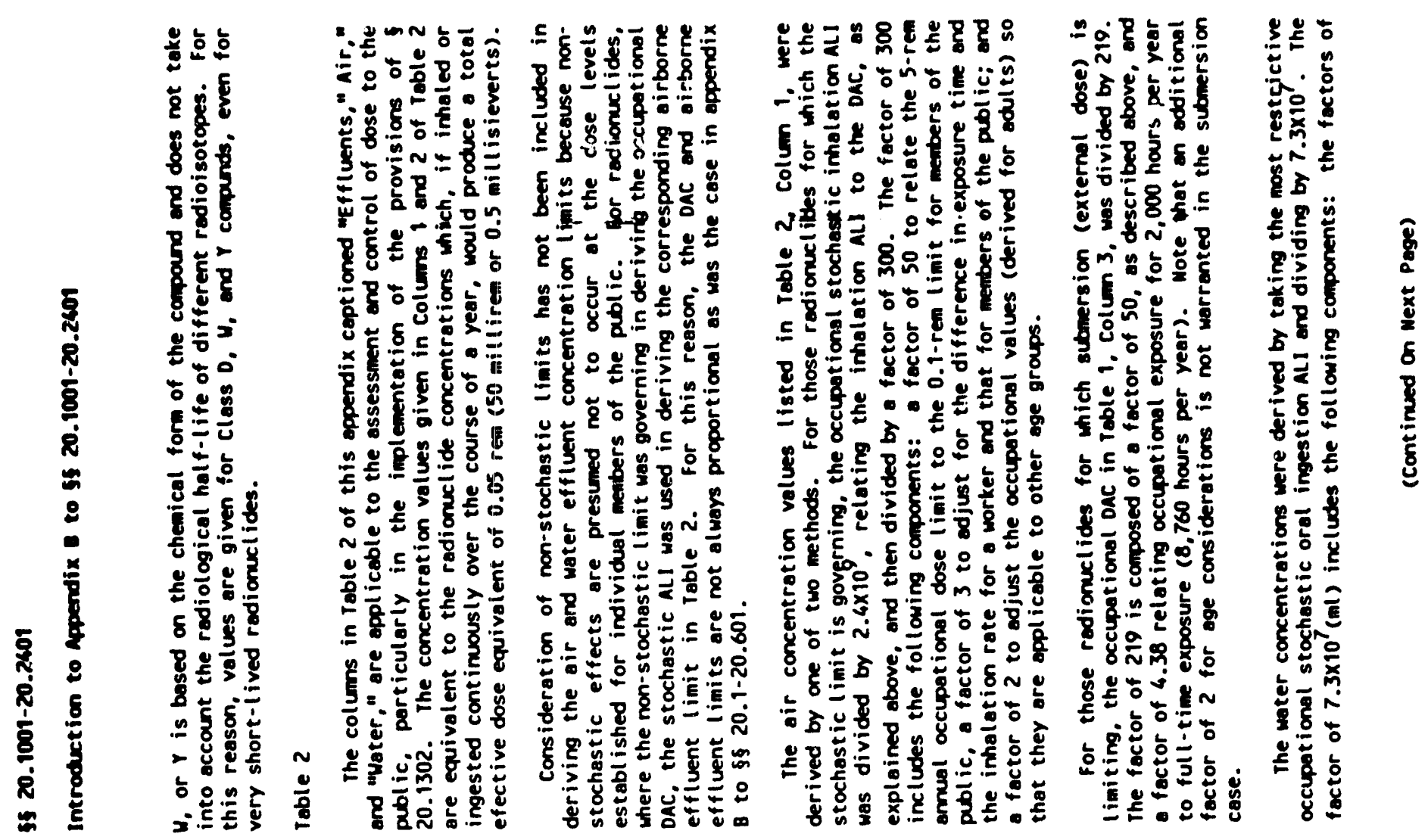


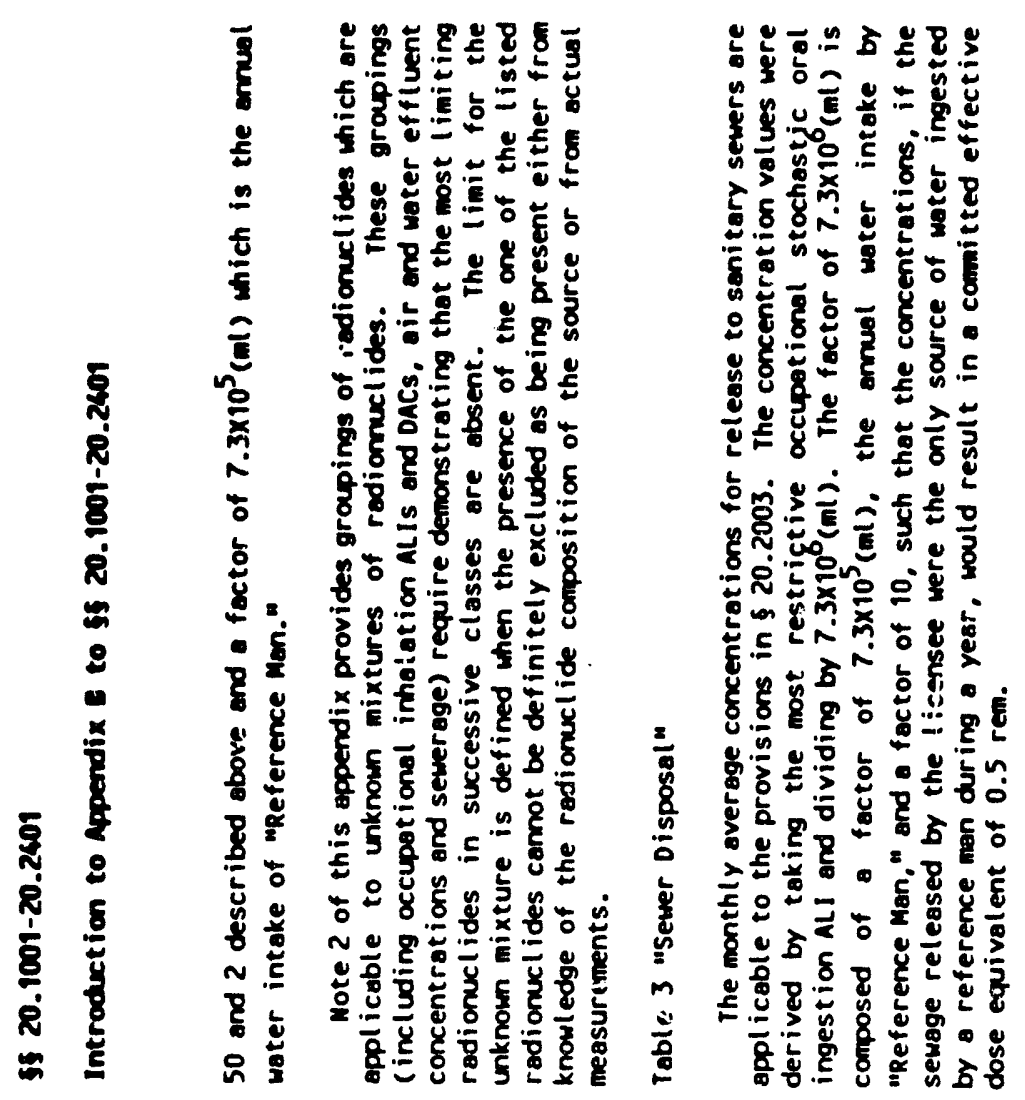




\section{REFERENCES}

1. Standards for Protection Against Radiation, 10 CFR Part 20, $5 \$ 20.1-20.601$.

2. Standards for Protection Aganist Radiation, 10 CFR Part 20, $\$ 520.1001-20.2401$. 


\section{EzERA! PRONISIOUS}

20.9

20.1 (a)

20.1 (b)

20.1 (c)

20.2

20.3

$20.3(a)(1)$

$20.3(a)(2)$

$20.3(0)(3)$

$20.3(0)(4)$

$20.3(0)(5)$

$20.3(0)(6)$

$20.3(0)(7)$

$20.3(0)(8)$

$20.3(8)(9)$

$20.3(8)(10)$

$20.3(a)(11)$

$20.3(a)(12)$

$20.3(a)(13)$

$20.3(8)(14)$

$20.3(0)(15)$

$20.3(a)(16)$

$20.3(a)(17)$

$20.3(a)(18)$

$20.3(a)(19)$

$20.3(a)(20)$

20.4

$20.4(0)$

$20.4(b)$

$20.4(c)$

$20.4(d)$

20.5

$20.5(a)$

20.6

20.7

20.8

$20.8(a)$

$20.8(b)$

$20.8(c)$
Purpose

ALARA

Scope

Definitions

Units of Radiation Dose

Units of rodiosctivity

Interpretations

Communications

Information collection

requirements: OMB approval

PERMISSIBLE DOSES, LEVELS AND CONCENTRATIONS

20.101

20.101(0)

Radiation dose standards for

individuals in restricted areas 29

20.101(b)

20.102

$20.102(a)(1)$

$20.102(a)(2)$

$20.102(b)$

$20.102(b)(1)$

$20.102(b)(2)$

$20.102(c)(1)$

$20.102(c)(2)$

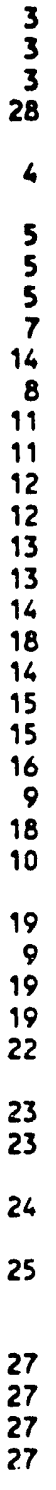


20.103

$20.103(0)(1)$

$20.103(a)(2)$

$20.103(a)(3)$

$20.103(b)(1)$

$20.103(b)(2)$

$20.103(\mathrm{c})$

$20.103(\mathrm{c})(1)$

$20.103(c)(2)$

$20.103(\mathrm{c})(3)$

$20.103(c)(6)$

$20.103(d)$

$20.103(e)$

$20.103(4)$

$20.103(9)$

.20 .104

$20.104(0)$

$20.106(b)$

$20.104(c)$

20.105

$20.105(a)$

$20.105(b)$

$20.105(c)$

20.106

$20.106(a)$

$20.106(b)$

$20.106(c)$

$20.106(d)$

$20.106(e)$

$20.106(f)$

$20.106(g)$

20.107

20.108

Exposure of individuals to concentrations of radionctive meteriols in oir in restricted arees.

29

31

34

Exposure of minors.

38

38

38

Permissible levels of radiation in unrestricted oreas.

Radioactivity in effluents to unrestricted oreas.

40
42
43
43
42
41
40
41

Medical diagnosis and therapy.

Orders requiring furnishing

of bio-essay services.

34

PRECAUTIONARY PROCEDURES

20.201

$20.201(0)$

$20.201(b)$

20.202

$20.202(a)$

$20.202(b)$

$20.202(b)(q)$

$20.202(b)(2)$

$20.202(b)(3)$

$20.202(c)$

$20.202(\mathrm{c})(1)$

$20.202(c)(2)$

\subsection{3}

$20.203(0)(1)$

$20.203(a)(2)$

$20.203(b)$

$20.203(\mathrm{c})(1)$

$20.203(c)(2)$
Surveys

Personnel monitoring

45

11

14

11

45

45

46

Caution signs, labels, signals and controls. 
S5 $20.1-20.601$

$20.203(c)(3)$
$20.203(c)(4)$
$20.203(c)(5)$
$20.203(c)(6)$
$20.203(c)(7)$
$20.203(d)(1)$
$20.203(d)(2)$
$20.203(e)(1)$
$20.203(e)(2)$
$20.203(f)(1)$
$20.203(f)(2)$
$20.203(f)(3)$
$20.203(f)(4)$

20.204

$20.204(a)$

$20.204(b)$

$20.204(c)$

$20.204(d)$

20.205

$20.205(0)(1)$

$20.205(a)(2)$

20.205 (b)(1)

$20.205(b)(2)$

$20.205(c)(1)$

$20.205(c)(2)$

20.205 (d)

20.206

20.207

20.207(a)

20.207 (b)

\section{MASTE DISPOSAL}

\subsection{1}

20.301(日)

$20.301(b)$

$20.301(c)$

20.302

$20.302(a)$

$20.302(b)$

20.303

$20.303(8)$

$20.303(b)$

$20.303(b)(1)$

$20.303(b)(2)$

$20.303(c)$

$20.303(d)$

20.305

20.306

$20.306(d)$
Caution sions: Exceptions

47

47

50

52

60

61

61

64

65

64

62

62

62

62

63

Procedures for picking up, receiving and opening packages.

66

66

66

67
67

67

67

Instruetion of personnel

39

storage and control of

licensed materials in unrestricted areas.

57

57
57

General Requirement 69

69

69

69

Methods for obtaining approval

of proposed disposal procedures. 70

70

70

Disposal by release into

sanitary sewerage systems. 71

71

71

71

71

71

71

Treatment of disposal by incineration.

72

Disposal of specific wastes. $\quad 73$ 
Transfer for disposal and manifests.

$20.311(a)$
$20.311(b)$
$20.311(c)$
$20.311(d)$
$20.311(e)$
$20.311(f)$
$20.311(g)$
$20.311(h)$

74

74

74

75

75

76

76

\section{RECORDS, REPORTS, AND NOTIFICATION}

20.401

$20.401(a)$

$20.401(b)$

$20.401(c)(1)$

$20.401(c)(2)$

$20.401(c)(3)$

$20.401(c)(4)$

$20.401(c)(5)$

20.402

$20.402(a)(1)$

$20.402(a)(2)$

$20.402(b)$

$20.402(c)$

$20.402(d)$

20.402 (e)

20.403

$20.403(a)$

$20.403(b)$

$20.403(c)$

$20.403(d)$

20.405

$20.405(0)(1)$

$20.404(8)(2)$

$20.405(b)$

$20.605(c)(9)$

$20.405(c)(2)$

$20.405(d)$

$20.405(e)$

20.407

$20.407(a)$

20.607(b)

20.408

$20.408(8)$

$20.408(b)$

20.409

$20.409(0)$

20.409 (b)
Records of surveys, radiation monitoring and disposal.

80

86

80

86

82

89

91

Reports of theft or loss of licensed material.

92

92

92

93

93

Notification of Incidents -

94

94

95

Reports of overexponsures and excessive levels and concentrations.

96

97

97

96

96

97

98

Persomel monitoring reports. $\quad 100$

100

100

Reports of personnel monitoring

on termination of employment or work. 102

102

102

Notification and reports to individusels. 
INDEX TO

S5 $20.1-20.601$

Extion nuber and subiest

Pene-lumer

20.501

Applications for exemptions.

105

20.502

Additional requirements.

105

20.601

violations.

106 


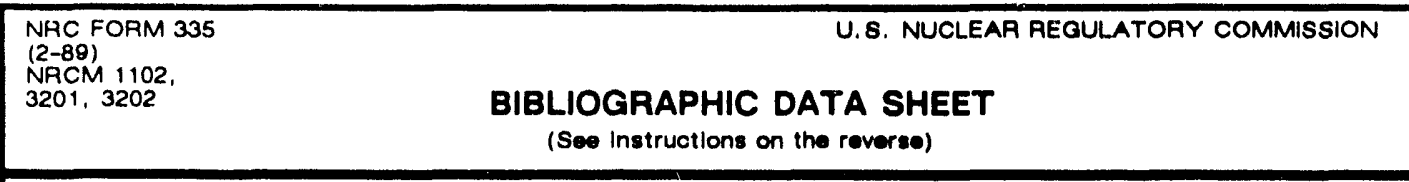

2. TITLE AND SUBTITLE

Standards for Protection Against Radiation-10 CFR Part 20: A Comparison of the Existing and Revised Rules

(See Instructions on the reverse)

3. DATE REPORT PUBLISHED

\begin{tabular}{c|c} 
MONTH & YEAR \\
Cutober & 1991
\end{tabular}

4. FIN OR GRANT NUMBER

5. AUTHOR(S)

D. A. Cool, H. T. Peterson, Jr.

6. TYPE OF REPORT

Technical

7. PERIOD COVERED (Inclusive Dates)

8. PERFORMING ORGANIZATION - NAME AND ADDRESS (If NRC, provido Divislon, Offlce or Reglon, U.S. Nuclear Regulatory Commission, and malling address; if contractor, provide name and malling address.)

Division of Regulatory Applications

Office of Nuclear Regulatory Research

U.S. Nuclear Regulatory Commission

Washington, DC 20555

8. SPONSORING ORGANIZATION - NAME AND ADDRESS (If NRC, type "Same as above"; If contractor, provide NRC Division, Office or Reglon, U.S. Nuclear Regulatory Commission, and malling address.)

Same as 8 . above

10. SUPPLEMENTARY NOTES

11. ABSTRACT (200 words or less)

On May 21, 1991, the Nuclear Regulatory Commission (NRC) issued a revision to its standards for protection against ionizing radiation, 10 CFR Part 20. Although the revised part $(\S \S 20.1001-20.2401)$ became effective on June 20, 1991, licensees may defer implementation of the revised rule until January 1, 1993. Licensees continue to be required to comply with the provisions of $\S \S 20.1-20.601$ until the time they adopt the provisions of $\S \S 20.1001-20.2401$. Therefore, between June 20, 1991 and January 1, 1993 both the provisions of $\S \S 20.1-20.601$ and $\S \S 20.1001-20.2401$ are in effect. This NUREG presents a comparative text of the provisions of the revised Part 20 ( $\S 20.1001-20.2401$ ) to the text of $\S \S 20.1-20.601$ for use by the NRC staff and NRC licensees.

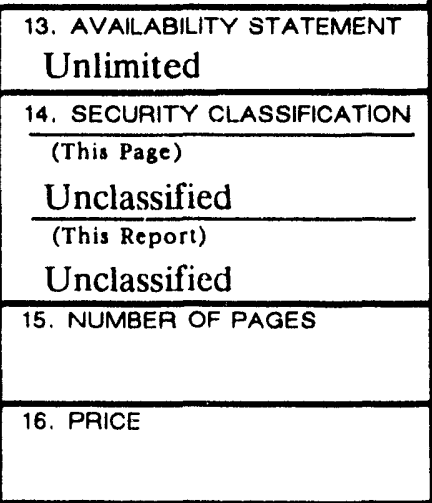




\section{$r$}
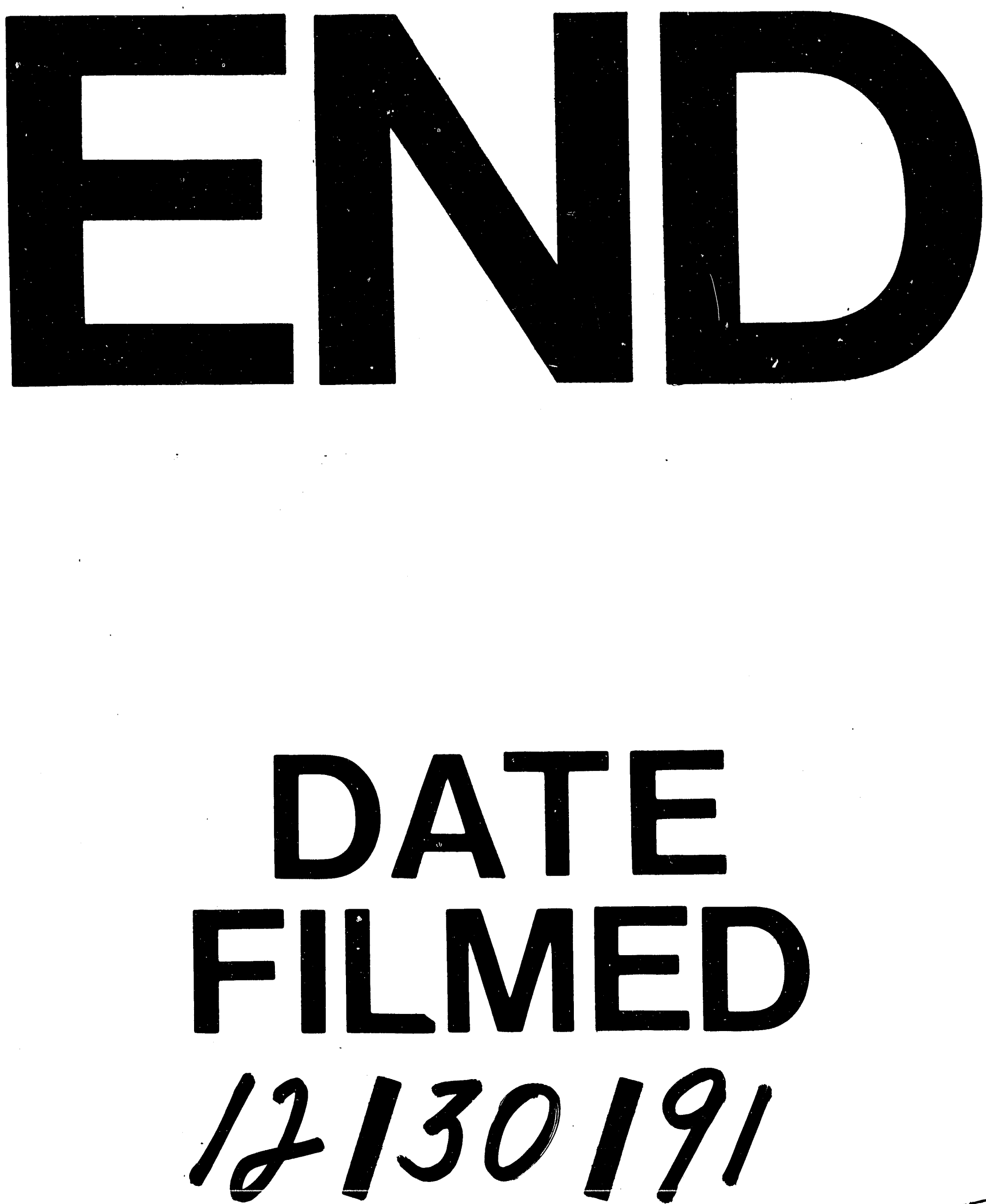
